\title{
Roles of miR-137 in Muscular Dystrophy and Muscular Dystrophy-Related Phenotypes in
}

\section{Drosophila melanogaster}

\author{
Doctoral Thesis
}

\author{
Dissertation for the award of the degree \\ "Doctor rerum naturalium (Dr. rer. nat.)" in \\ the GGNB program: “Genes \& Development” of \\ the Georg August University Göttingen \\ Faculty of Biology
}

submitted by

Shruti Chhetri

born in Kathmandu, Nepal

Göttingen, March 2019 


\section{Members of the Thesis Committee}

\section{Thesis Committee Members}

Prof. Dr. Halyna Shcherbata (Supervisor, reviewer)

Max Planck Research Group for Gene Expression and Signaling

Max Planck Institute for Biophysical Chemistry

Am Fassberg 11, 37077 Göttingen, Germany

Prof. Dr. Stefan Bonn ( $2^{\text {nd }}$ Reviewer)

The Center for Molecular Neurobiology Hamburg (ZMNH)

Institute of Medical Systems Biology

Martinistr. 85, 20251 Hamburg, Germany

Prof. Dr. Jörg Großhans

University Medical Center Göttingen

Department of Developmental Biochemistry

Justus-von-Liebig-Weg 11, 37077 Göttingen, Germany

\section{Extended Thesis Committee Members}

Dr. Roland Dosch

University Medical Center Göttingen

Department of Developmental Biochemistry

Justus-von-Liebig-Weg 1137077 Göttingen Germany

Prof. Dr. Martin Göpfert

Schwann-Schleiden Research Centre

Department of Cellular Neurobiology

Julia-Lermontowa-Weg 3, 37077 Göttingen, Germany

Prof. Dr. Ahmed Mansouri

Max Planck Institute for Biophysical Chemistry

Molecular Cell Differentiation Group

Am Fassberg 11, 37077 Göttingen, Germany 


\section{Affidavit}

I, Shruti Chhetri, confirm that the work presented in this thesis is my own. This thesis was written independently and no other sources or aids were used except the mentioned ones. This thesis has not already been published and is also not concurrently submitted for any other degree.

Göttingen, 01.03.2019 


\section{Table of contents}

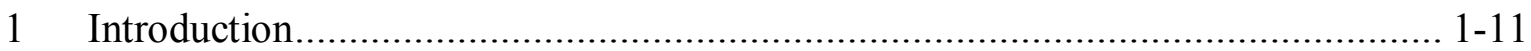

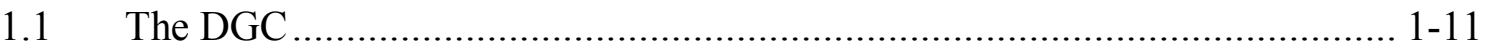

1.2 Drosophila as a model for Muscular Dystrophy ........................................... 1-14

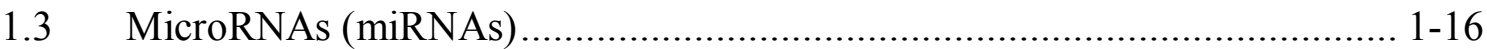

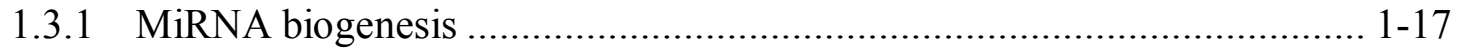

1.3.2 MiRNA mode of action .................................................................... 1-18

1.3.3 MiRNA target identification and seed sequence ...................................... 1-19

1.3.4 MiRNAs role in gene regulation ....................................................... 1-20

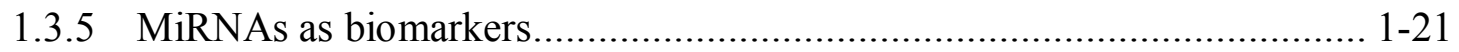

1.3.6 MiRNAs as stress regulators ………………...................................... 1-22

$1.4 \quad$ Stress and Muscular Dystrophies ……………......................................... 1-23

1.5 MiRNAs profiles in Muscular Dystrophies ................................................. 1-23

$1.6 \quad$ Architecture of adult Drosophila muscle...................................................... 1-24

$1.7 \quad$ Sterility and Muscular Dystrophy ............................................................. 1-26

1.7.1 Spermatogenesis in Drosophila melanogaster ......................................... 1-27

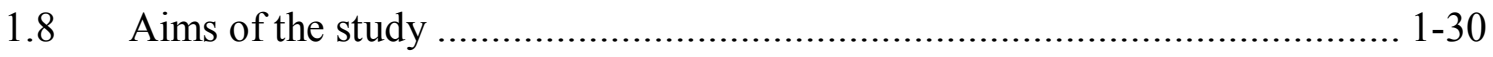

2 Materials and Methods ............................................................................... 2-31

$2.1 \quad$ Fly work

2.1.1 Fly stocks and maintenance ........................................................... 2-31

2.1.2 Standard Drosophila food media........................................................... 2-32

2.1.3 Temperature and nutritional stress ....................................................... 2-32

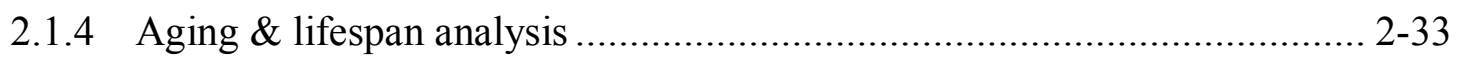

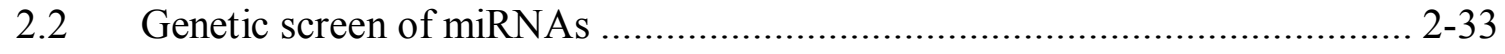

$2.3 \quad$ Muscle Analysis ............................................................................. 2-34

2.4 Phenotypic Classification.................................................................... 2-34

2.4.1 Muscle Degeneration Phenotypes ...................................................... 2-34

2.4.2 Septate junction phenotype ……………............................................ 2-34

2.5 Gene ontology analysis of predicted miRNA targets ................................... 2-35

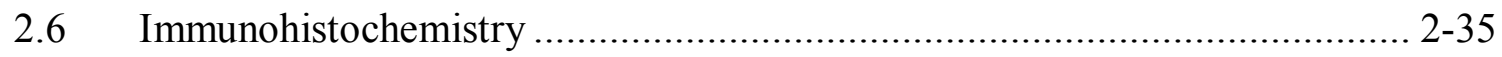

2.6.1 Permeability assay ........................................................................... 2-36

2.7 In situ hybridization (ISH) .................................................................. 2-36

2.7.1 Fluorescence in situ hybridization (FISH) …………............................... 2-37

2.8 Genomic DNA extraction from fly leg and the whole fly .............................. 2-38 
2.9 Polymerase chain reaction (PCR) ...................................................... 2-38

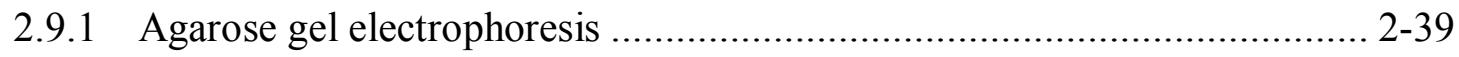

2.10 RNA extractions and cDNA synthesis .................................................. 2-39

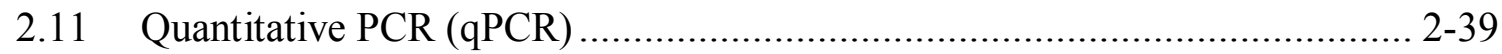

2.11.1 Quantitative miRNA expression analysis ...................................... 2-40

2.12 Transfection of Drosophila cell lines (S2R+ cell lines) .............................. 2-40

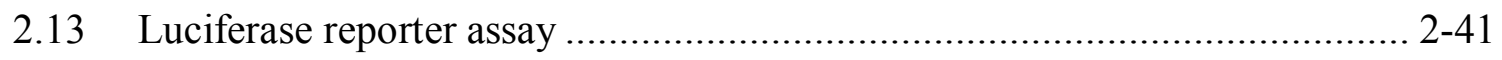

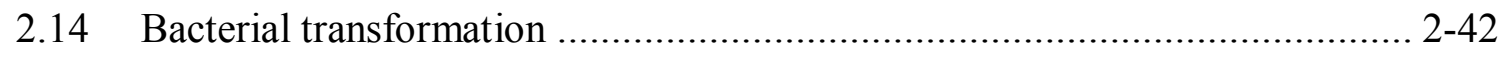

2.15 Midi-preparation of plasmid DNA …................................................... 2-42

2.16 Image processing and quantification ................................................... 2-42

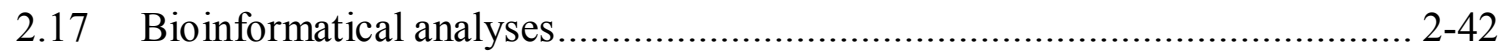

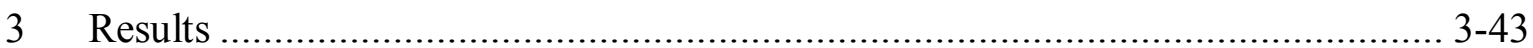

3.1 Screen of miRNAs that are predicted to target the DGC ............................. 3-43

3.1.1 The DGC components are predicted to be targeted by multiple miRNAs .. 3-43

3.1.2 MiRNA mutants have deregulated mRNA levels of $D g, D y s$, and Syn 1.... 3-46

3.1.3 Loss of miRNA causes muscle degeneration phenotypes ....................... 3-47

3.2 Validation of muscle degeneration due to miRNA loss ............................ 3-49

3.3 Conserved predicted targets of miRNAs are associated with multiple biological

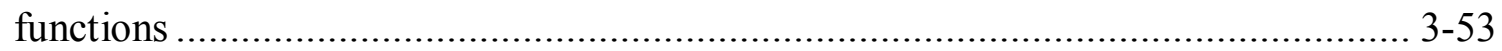

3.4 Ectopic expression of candidate miRNA affects muscle maintenance ........... 3-55

3.5 Candidate miRNAs target Dg-3' UTR in vitro ........................................ 3-57

3.6 Downregulation of Dg affects muscle maintenance................................... 3-59

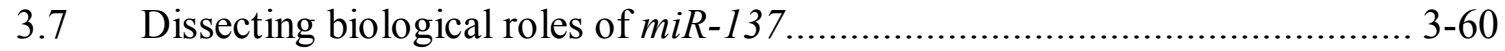

3.7.1 MiR-137 is conserved among higher eukaryotes .................................... 3-61

3.7.2 MiR-137 is expressed in larval muscle, brain, and testis ......................... 3-61

3.7.3 MiR-137 affects muscle maintenance .................................................... 3-64

3.7.4 MiR-137 mutants have perturbed spermatogenesis ................................ 3-68

3.7.5 MiR-137 is essential to maintain permeability barrier........................... 3-72

3.7.6 MiR-137 mutants have abnormal septate junction (SJ) morphology .......... 3-74

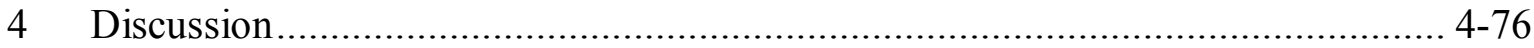

4.1 MiRNAs are required for muscle maintenance....................................... 4-77

4.2 MiR-137 is required cell autonomously for muscle maintenance .................. 4-80

4.3 MiR-137 is required to maintain a precise level of $\mathrm{Dg}$ in adult muscle .......... 4-81

4.4 MiR-137 is required in somatic cells to maintain permeability barrier .......... 4-83 
4.5 Dg levels must be regulated to maintain the permeability barrier ................. 4-84

4.6 MiR-137 acts in soma to regulate $\mathrm{Dg}$ in septate junctions .......................... 4-84

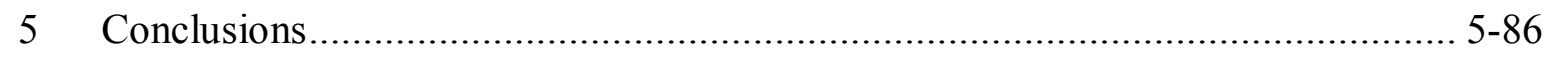

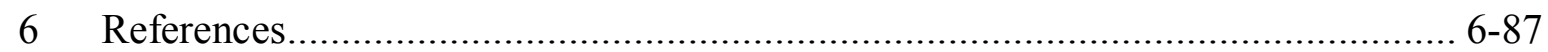

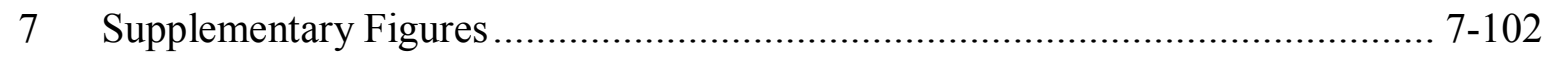

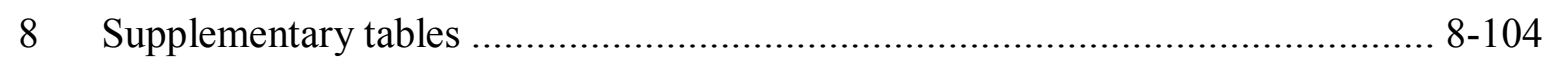

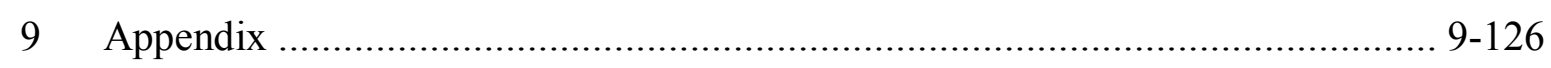




\section{List of figures}

Figure 1. The Dystrophin Glycoprotein Complex (DGC) and its associated components in

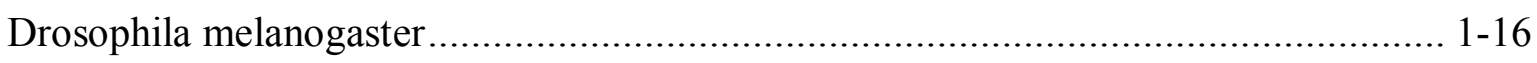

Figure 2. The canonical pathway of miRNA biogenesis ..................................... 1-18

Figure 3. Drosophila Indirect Flight Muscles .................................................. 1-26

Figure 4. Spermatogenesis in adult Drosophila melanogaster ................................. 1-29

Figure 5. Schematic representation of luciferase assay .......................................... 2-41

Figure 6. Multiple miRNAs are predicted to target the DGC components................... 3-45

Figure 7. Candidate miRNA mutants have deregulated mRNA levels of the DGC

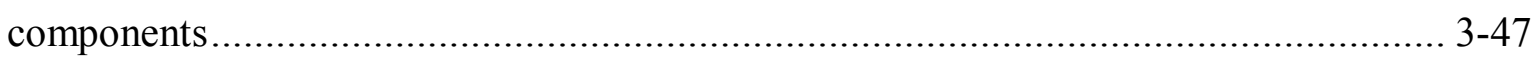

Figure 8. MiRNA mutants show muscle degeneration phenotype that is enhanced upon stress

Figure 9. Loss of miRNA affects muscle maintenance .......................................... 3-52

Figure 10. GO term for component processes for each miRNA targets ....................... 3-54

Figure 11. Over-expression of miR-137 results in strong muscle degeneration and muscle

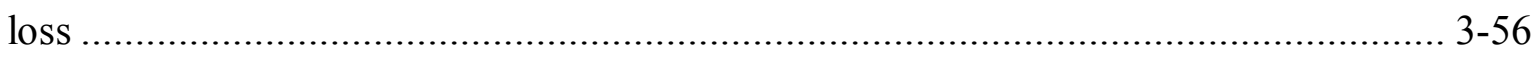

Figure 12. Over-expression of candidate miRNAs affects muscle maintenance ........... 3-57

Figure 13. Selected miRNAs can target Dg in vitro ............................................... 3-58

Figure 14. Downregulation of $\mathrm{Dg}$ shows age-dependent loss of muscle integrity .......... 3-60

Figure 15. MiR-137 has conserved seed as well as mature miRNA sequences ............. 3-61

Figure 16. MiR-137 expression patterns ........................................................... 3-63

Figure 17. MiRNA-137 regulates Dg levels for muscle maintenance........................... 3-65

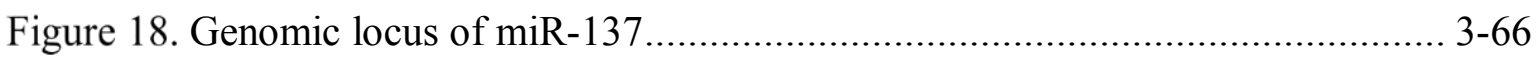

Figure 19. Dg is a bona fide target of miR-137 in muscle ...................................... 3-67

Figure 20. MiR-137 ${ }^{\mathrm{ko}}$ mutants have an increase in somatic cell population.................. 3-69

Figure 21 . The early somatic cell population is maintained by downregulating Dg in miR-

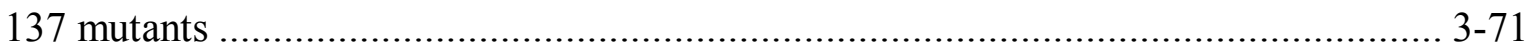

Figure 22. Permeability barrier is maintained by miR-137 .................................. 3-73

Figure 23. MiR-137 mutants have defective septate junction phenotype ..................... 3-75

Figure 24. Mode of action of miR-137 in muscle ..................................................... 4-82

Figure 25. Mode of action of miR-137 in testis ................................................ 4-85 


\section{List of supplementary figures}

Supplementary Figure 1. Over-expression of Dg in muscle during development results in

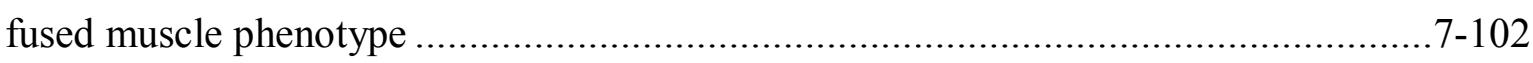

Supplementary Figure 2. MiR-137 mutants have delayed in differentiation ................ 7-102

Supplementary Figure 3. Lifespan analysis on miRNA mutants ............................... 


\section{List of tables}

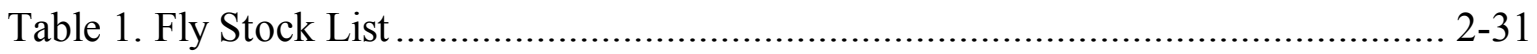

Table 2. Antibodies used for immunohistochemistry …........................................... 2-35

Table 3. Duration of tissue fixation and permeabilization ........................................ 2-37

Table 4. Conditions used for genomic DNA extraction........................................... 2-38

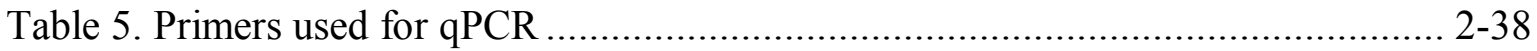

\section{List of supplementary tables}

Supplementary Table 1. The DGC components of Drosophila and its functions $8-104$

Supplementary Table 2. Relative transcript levels of $D g$, Dys, and Syn1 due to miRNA loss $8-108$

Supplementary Table 3. Percentage of muscle degeneration in miRNA mutants. 8-109

Supplementary Table 4. Percentage of muscle degeneration in selected miRNA mutants . 8112

Supplementary Table 5. Candidate miRNAs can target $D g$ in vitro $8-121$

Supplementary Table 6 . Relative $D g$ mRNA levels in adult fly muscle and testes $8-122$

Supplementary Table 7. Early somatic cell counts per testes $8-124$

Supplementary Table 8. Early somatic cell counts at the apical portion of testes $8-124$

Supplementary Table 9. Percentage of permeable testes . $8-125$

Supplementary Table 10. SJ counts and morphology on elongated spermatids $8-125$ 


\section{Abstract}

Muscular dystrophies (MDs) are a group of diseases that cause muscular and neurological disorders in human patients. They are associated with a multi-component complex called the Dystrophin Glycoprotein Complex (DGC). The DGC connects the extracellular matrix to the cytoskeleton and is well-conserved in animals. Perturbation of this complex is associated with various kinds of MDs, leading to a diverse range of muscle and nervous system abnormalities. Dystroglycan (Dg) is a central DGC component, mutations of which are associated with a heterogeneous group of MDs also known as dystroglycanopathies.

MiRNAs are small, noncoding RNAs that function in posttranscriptional gene regulation and often represses their target mRNAs. Previous work has shown that similar to MD, stress itself causes muscle degeneration, and altered miRNA expression profiles have been detected in dystrophic as well as stressed wild type flies. These results indicate that miRNAs influence a common regulatory pathway between stress and MD. Though much is known about the DGC and its relevance to MDs, the molecular and genetic pathways underlying MD pathogenesis remain largely unknown.

To understand the role of miRNAs in DGC signaling and their contribution to MDs, in particular during stress, we screened several miRNAs that are predicted to target multiple components of the DGC study their potential roles in MD development, particularly upon various stresses. We found that $m i R-137, m i R-966$, and $m i R-927$ affect muscle integrity upon stress and aging. Our study further reveals that miR-966 and $m i R-137$ are required more during adult muscle maintenance than developing muscles. MiR-137, in particular, is a stress-responsive miRNA, as the severity of the phenotypes related to muscle maintenance progressed in a stress- and age-dependent manner.

We further show that levels of Dg must be regulated to sustain healthy muscle, and this regulation includes targeting of $D g$ by $m i R-137$. The $D g-m i R-137$ interaction is required to address negative effects of stress in adult muscle maintenance. Our results also demonstrate that a perturbed blood-testis barrier (BTB) in testes is a novel phenotype related to MD, and miR-137 regulates the expression of $D g$ in early somatic cells of Drosophila testes to maintain the BTB. Our results highlight the importance of miRNAs in the regulation of the DGC and MD, particularly on muscle maintenance that is accelerated upon stress. 


\section{Introduction}

Muscular Dystrophies (MDs) are a group of genetic disorders mostly characterized by progressive muscle degeneration and wasting. The condition often begins by affecting a particular group of muscles, such as the limb, facial, and axial muscles, as well as respiratory and cardiac muscles, before affecting the overall musculature to variable degrees. In some cases, the disorder can affect other tissues such as the brain, inner ear, eye, or even skin. More than 30 different types of MDs have been characterized so far. The severity, age of onset, consequences, and disease progression vary from patient to patient, as well as from the type of disorder. Unfortunately, there is neither a cure nor adequate treatments for this group of diseases, making it more critical to understand disease-specific complications and pathogenesis as well as the implementation of medical-related advances. Duchenne muscular dystrophy (DMD) is the most severe type of MD. It affects $1 / 3500$ males worldwide and is an X-linked, fatal disorder. Loss of Dystrophin (Dys) is associated with DMD. Patients with DMD die in their early twenties because of respiratory or cardiac failure (Durbeej and Campbell, 2002). Dys is also associated with a less severe form of MD called Becker MD (BMD), which also affects males with mean age of onset of 12 years old, resulting in a loss of ambulation and cardiac defects (Wilson et al., 2017). Dys is the largest gene in the human genome at $2.5 \mathrm{Mb}$ and is a part of a membrane-associated protein complex called the Dystrophin Glycoprotein Complex (DGC) (Hoffman et al., 1987; Kunkel et al., 1986). Mutation in any of the components in the DGC (Chapter1.1) is associated with various kinds of MDs, namely limb-girdle MD (LGMD), congenital MD (CMD), DMD, BMD, muscle-eyebrain disease (MEB), Walker-Warburg syndrome (WWS), and myotonic dystrophy. All of these diseases share the common symptoms of muscle degeneration, reduced lifespan, cardiomyopathy, as well as some extent of neuronal disorders. The involvement of the DGC in MDs, affecting various tissues causing individual symptoms are due to mutations in different proteins of the DGC that share similar cellular functions. Most of the components of the DGC are well-conserved throughout the animal kingdom and are well-characterized, making them easier to study in different model organisms in order to highlight their molecular function and regulation in the disease state.

\subsection{The DGC}

The DGC is a large, oligomeric complex that connects the extracellular matrix to the cytoskeleton. In mammals, it is composed of transmembrane dystroglycan ( $\alpha$ - and $\beta$-), 
sarcoglycans ( $\alpha-, \beta-, \gamma-$, and $\delta$-), cytoplasmic dystrophin, syntrophins ( $\alpha 1-, \beta 1-, \beta 2-, \gamma 1-$, and $\gamma 2-), \alpha$-dystrobrevin, and neuronal nitric oxide synthase (Durbeej and Campbell, 2002). $\alpha$ and $\beta$-dystroglycan connect the extracellular matrix (ECM) component laminin-2 to the cytoskeleton via dystrophin, which in contractile muscle cells accounts for the mechanical stress resistance and the stability of the muscle sarcolemma (Ervasti and Campbell, 1993). Thus, the DGC in muscles has very important roles: 1) to account for the flexibility and the durability of the resilient plasma membrane to maintain its structure in each contraction and retraction; and 2) to act as a signal transduction platform to maintain the link between the inner and outer environments of the cell. Syntrophins, having various protein-protein interaction motifs, are famous as adaptor proteins capable of binding to heterotrimeric $\mathrm{G}$ proteins, adaptor protein Grb2, and neuronal nitric oxide synthase (nNOS) (Cacchiarelli et al., 2010; Xiong et al., 2009; Zhou et al., 2006) among other signaling molecules. Recently it has been shown that $\alpha$-syntrophin can bind directly to multiple spectrin-like repeats in dystrophin and mediate its binding to nNOS (Adams et al., 2018).

The DGC components are associated with various forms of MDs. $\alpha 2$-laminin is associated with CMD, sarcoglycan deficiency is linked to LGMD, and hypogycosylation of dystroglycan is associated with severe forms of congenital (Fukuyama CMD, FCMD; WWS; CMD type 1C/1D, MDC1C/MDC1D), and late-onset muscular dystrophies (MEB; hereditary inclusion body myopathy (HIBM)) (Cohn, 2005). Progressive muscle degeneration is a hallmark of many of these MDs; however, clinical traits for these group of diseases are not limited to the muscles. They are also associated with structural brain defects, abnormal neuronal migration, as well as mental retardation (Balci et al., 2005; Muntoni et al., 2002; van Reeuwijk et al., 2006; Waite et al., 2012; Zhou et al., 2006). The main classes of proteins involved in MDs can be subdivided into groups: 1) extracellular matrix proteins, or external membrane proteins (laminin, collagen VI);2) enzymes or proteins presumably with enzymatic function that are either involved in glycosylation of $\alpha$-Dystroglycan, and those that are not involved in glycosylation of $\alpha$-dystroglycan; 3) sarcolemma-associated proteins; a major subcomplex of the DGC (dystroglycan, dystrophin, and sarcoglycans); 4) nuclear membrane proteins (lamin A or C, emerin etc.), sarcomeric proteins (titin), and others (DUX4) (Mercuri and Muntoni, 2013).

MDs, in general, affect various types of tissues causing individual symptoms among patients. Moreover, the genetic and molecular pathways underlying MDs' pathogenesis remain poorly understood. Recent advances in the understanding of MDs' pathogenesis suggests that variable symptoms of MDs are due to the different components of the DGC and their isoforms 
being expressed in many tissues. For example; Apo-dystrophins- 1 and 3 are regulated by a promoter situated between the exons 62 and 63 of the dystrophin gene. It is expressed in tissues such as brain, lung, liver, and kidney. Apo-dystrophin-2 is regulated by a promoter positioned between exons 55 and 56 of the dystrophin gene and is expressed in peripheral nerves. $\alpha$ - and $\beta$-dystroglycan are also expressed in the brain, lung, liver, and kidney (Tinsley et al., 1994). Each of the five homologous isoforms of syntrophins has a unique tissue expression. $\alpha 1$-syntrophin is primarily expressed in skeletal muscles but also in heart, brain, and other mammalian tissues; $\beta 1$ - and $\beta 2$-syntrophins are broadly distributed; $\gamma 1$ - and $\gamma 2$ syntrophins are highly expressed in brain, but the $\gamma 2$ isoform also has a broader distribution in mammalian tissues (Bhat et al., 2013).

Dystroglycan is one of the essential components of the DGC. Dg has two subunits, $\alpha$ - and $\beta$ dystroglycan. $\alpha$-dystroglycan connects extracellular matrix proteins to the muscle sarcolemma, and $\beta$-dystroglycan is a transmembrane subunit connecting $\alpha$-dystroglycan to various cytoskeletal adaptor proteins, such as dystrophins and syntrophins. Defects in glycosylation of $\alpha$-dystroglycan is one of the major causes of CMD and LGMD. Disorders due to mutations in dystroglycan, or in the genes encoding the proteins and enzymes involved in the glycosylation of $\alpha$-dystroglycan are collectively known as dystroglycanopathies. The dystroglycanopathies are described as a group of diseases caused by the loss or reduced binding of $\alpha$-dystroglycan to its extracellular ligands, such as laminin, agrin, neurexins, perlecans, pikachurin, and Slit (Brown and Winder, 2017). Mutations in these proteins share the clinical features of dystroglycanopathies, which widens the horizons of how crucial and selective the role of $\mathrm{Dg}$ is in various kinds of MDs. $\alpha$-Dystroglycan is glycosylated mostly by O-mannosylation (Mercuri and Muntoni, 2013). Glycosylation overall has many enzymatic steps that are regulated during development and in a tissue-specific manner. $\alpha$-dystroglycan glycosylation has a fundamental role in muscles as well as in basal membrane maintenance (Jimenez-Mallebrera et al., 2009). It is also required for the development of the central nervous system, as many MD patients experience cognitive impairment and learning disability and develop behavioral and neuropsychotic disorders (Waite et al., 2012).

Neuromuscular junction (NMJ) shares a structural function, by stabilizing the muscle sarcolemma from various excitations and contractions through the coupling from neurons. Many motor neuron disorders such as in spinal muscular atrophy (SMA) and amyotrophic lateral sclerosis (ALS) can cause progressive degeneration of muscle fibers which occurs due to loss of innervation in muscle tissues (Kreipke et al., 2017). Dystrophin is found at extrasynaptic and synaptic regions of muscle fibers and is required for NMJ development, 
and Dystroglycan is required for synaptic maturation as well as synaptic Utrophin, Laminin $\alpha 5$ and Laminin $\gamma 1$ concentration (Grady et al., 2000). In Drosophila, proper localization of glutamate receptors is mediated by the binding of Dg to Coracle (Cora) (Bogdanik et al., 2008; Marrone et al., 2011b).

In mammals, it has been shown recently that Dystroglycan sequesters phosphorylated Yap (Yes-associated protein) to prevent the actions of activating phosphatase as a mechanism to regulate cardiomyocyte proliferation (Morikawa et al., 2017), broadening the role of Dystroglycan in muscle maintenance and muscle-related diseases. Trim32 (tripartite motifcontaining protein 32), a ubiquitin ligase, is critical for muscle atrophy. Mutations in the third repeat of Trim 32 cause LGMD -2H (Frosk et al., 2002; Shieh et al., 2011), and inhibition of Trim32 enhanced plakoglobin binding and induced fiber growth, while downregulation of plakoglobin caused muscle atrophy (Cohen et al., 2014). Since muscle weakness is often due to muscle atrophy, hypertrophy, or both, as seen in DMD patients, it is important to investigate the relationship between Trim-32, plakoglobin, and the DGC to identify further players in MDs and their disease relevance.

\subsection{Drosophila as a model for Muscular Dystrophy}

Drosophila melanogaster has many advantages as a model organism. Besides the relatively low cost of the cultivation, the forward and reverse genetic tools in Drosophila are much more advanced and sophisticated than in many other model organisms. The relatively fast life cycle ( $\sim 9$ days in ambient temperature and humidity), and short lifespan ( 3 months) of Drosophila makes it easier to cultivate large numbers of flies in a short amount of time, which is a great advantage in studying the developmental aspects of disease progression, as well as in creating a large amount of basic material required for biochemical and molecular assays. There are fly homologs of more than $75 \%$ of human genes that are associated with various kinds of disorders ranging from bacterial infections, metabolic disorders to aging, and cancer. Therefore, Drosophila melanogaster is an ideal model for studying the DGC, identifying its novel functions, interacting components, and factors involved in the physiological and molecular dynamics of its signaling and regulatory systems. Many of the core components of the DGC are evolutionarily conserved but with less diversity. Drosophila has only two syntrophins: syntrophin-like-1 (Syn1) homologous to $\alpha 1 / \beta 1 / \beta 2$-syntrophins, and syntrophin-like-2 (Syn2) homologous to $\gamma 1 / \gamma 2$-syntrophins in mammals. Dystrophin (Dys) is a sole homolog to mammalian utrophin and dystrophin, and a single copy of Dystrobrevin 
(Dyb) is homologous to $\alpha-$, and $\beta$-dystrobrevin in mammals (Greener and Roberts, 2000). As in mammals, DGC components in Drosophila (Figure 1) are expressed not only in the muscle but also in the nervous tissues (Bhat et al., 2013; Bogdanik et al., 2008; Deng et al., 2003; Marrone et al., 2011a; Shcherbata et al., 2007; van der Plas et al., 2006; Yatsenko et al., 2007). Many of the MD-related phenotypes in the muscle and nervous systems reported in mammals can be easily phenocopied in flies. Flies lacking $D g$ or Dys (further dystrophic flies) experience a shortened lifespan, age-dependent muscle degeneration, decreased mobility, and defective photoreceptor path-finding (Shcherbata et al., 2007), hyperthermic seizures (Marrone et al., 2011b), as well as decrease in presynaptic glutamate release at neuromuscular junctions (NMJs) (Bogdanik et al., 2008; van der Plas et al., 2006). Both $D g$ and Dys are required in both glial cells and neurons for correct neuronal migration (Muntoni et al., 2002; Shcherbata et al., 2007). In recent years, the roles of the DGC have not been limited to muscle or nervous tissues. Studies have shown that lack of the DGC complex, in particular $\mathrm{Dg}$, in patients is associated with elevated levels of creatine kinase in the blood, ataxic gait, learning disabilities, dilated cardiomyopathy, complete lissencephaly (type II), and autism spectrum disorder and are diagnostic features of dystroglycanopathies (Astrea et al., 2018; Bonnemann et al., 2014), broadening the horizon of Drosophila as a model organism to study the pathogenesis of MDs.

DMD is the most severe form of MD and has been studied quite extensively. As a consequence of muscle fiber damage in DMD, specific muscle-microRNAs (myo-miRs) are found to be released into the bloodstream of DMD patients, as well as in mammalian model of DMD ( $m d x$ mouse), and their levels correlate with the severity of the disease (Cacchiarelli et al., 2011b). The same study also proposed $m i R-1, m i R-133$, and $m i R-206$ as valuable biomarkers for the diagnosis of DMD. In fly models of cobblestone lissencephaly (similar to type II lissencephaly in humans), $m i R-310 \mathrm{~s}$ has been reported to play an important role as a buffering agent to establish the proper level of $D g$ level by targeting its alternative 3'untranslated region (3'UTR) (Yatsenko et al., 2014). The same miRNA is also known to play an important role in Hedgehog signaling in response to nutritional changes (Cicek et al., 2016). Similarly, $m i R-9 a$ has been shown to target $D g$ to maintain the precise level of $D g$ to establish myotendinous junction (MTJ) formation, and flies lacking $m i R-9 a$ have defective muscle architecture (Yatsenko and Shcherbata, 2014). Overall, miRNAs targeting the DGC can influence many signaling pathways, illustrating a molecular mechanism by which miRNAs serve as a quick and robust response in many signaling pathways. These studies show that miRNAs play a fundamental role in MDs. 


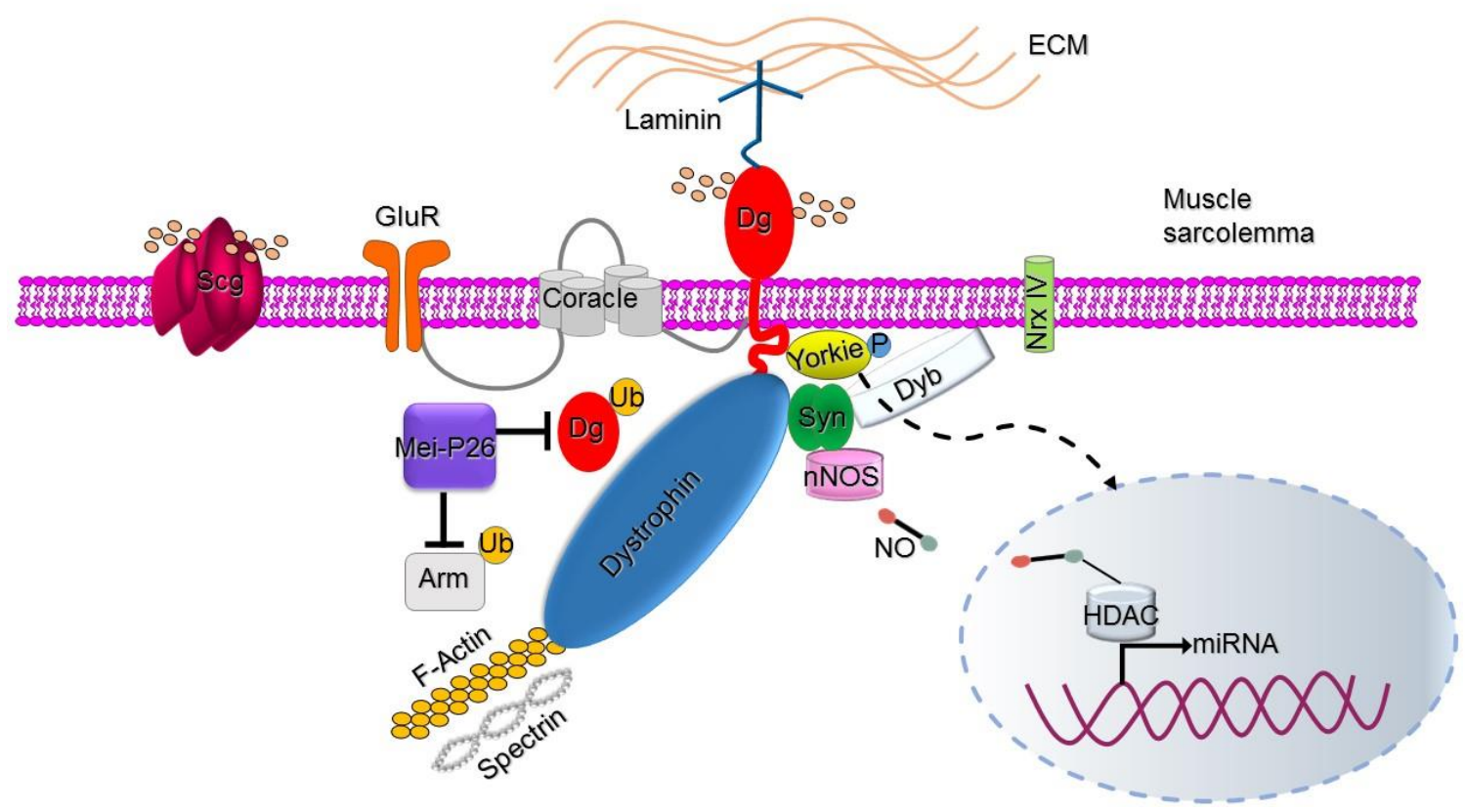

Figure 1. The Dystrophin Glycoprotein Complex (DGC) and its associated components in Drosophila melanogaster

The transmembrane protein Dystroglycan $(\mathrm{Dg})$ is a key component of the complex connecting the extra and intracellular environment by binding to laminins extracellularly and Dystrophin (Dys) intracellularly. The DGC acts as a scaffold for many signaling molecules such as syntrophin (Syn) and neuronal nitric oxide syntase (nNOS). nNOS produces nitric oxide (NO) which is involved in nitrosylation of histone deacetylases (HDACs), which in turn influences the gene expression. At the neuromuscular junction (NMJ), Dg is required for proper localization of glutamate receptor (GluR) which is mediated by Dg binding to Dys and coracle (Cora). Similar to the mammalian model, Dg sequester phosphorylated Yorkie (fly ortholog to Yap), and can influence muscle maintenance. Similarly, we also hypothesize that mei-P26 (Trim-NHL protein in fly) can ubiquitinate arm (armadillo, fly ortholog of plakoglobin) or Dg, promoting the DGC stability in skeletal muscle.

\subsection{MicroRNAs (miRNAs)}

MiRNAs are small, $\sim 22 \mathrm{bp}$ long, endogenous, non-coding RNA molecules that regulate gene expression post-transcriptionally in diverse cellular and developmental processes in a tissuespecific manner. They bind to 3'UTRs of targeted messenger RNAs (mRNAs) with partial complementarity and mediate gene expression via translation inhibition or mRNA decay (Bazzini et al., 2012; Djuranovic et al., 2012; Guo et al., 2010). Under certain conditions, they are also known to activate gene expression (Vasudevan, 2012). The miRNA field is relatively new with the discovery of the first miRNA just over two decades ago in the relatively simple eukaryote C. elegans (Lee et al., 1993; Wightman et al., 1993). They are the most abundant non-coding gene family, distributed widely in plants and animals. Since 
the discovery, 253 miRNAs in C.elegans, 258 miRNAs in Drosophila, and 1917 miRNAs in humans have been annotated so far (www.miRBase.org, as of 2/11/2018). The number of miRNAs present in a species has been shown to positively correlated with organismal complexity (Grimson et al., 2008), suggesting that miRNA-dependent fine-tuning of gene expression was necessary for the evolution of higher organisms (Heimberg et al., 2008). MiRNAs' functions can be extended from fine-tuning effects to significant alterations in the gene expression profile. They are known to control basic cellular processes such as cell growth, differentiation, proliferation, and apoptosis (Dhahbi, 2014), or to moderate physiological processes such as cell signaling, immune responses, tumorigenesis, development, and non-neoplastic disease pathogenesis (Koturbash et al., 2011). Some miRNAs are conserved in both their sequences and expression patterns across a wide range of animals, making them excellent models to better understand how similar processes are controlled in various organisms.

\subsubsection{MiRNA biogenesis}

MiRNA biogenesis begins with transcription by RNA polymerase II (Pol II), giving rise to a single-stranded RNA molecule called a primary miRNA (pri-miRNA) that is $\geq 1 \mathrm{~kb}$ long and has a stem-loop structure (Figure 2). Many pri-miRNAs are 3'polyadenylated and 5'capped, similar to the mRNAs transcribed by the same RNA Pol II enzyme (Lee et al., 2004; Winter et al., 2009). Pri-miRNAs then get processed by the Microprocessor Complex, which consists of the ribonuclease III enzyme, Drosha, and the RNA binding protein, Pasha (DGCR8 in mammals) (Denli et al., 2004; Ha and Kim, 2014). This complex further cleaves the hairpin resulting in the formation of a precursor-miRNA (pre-miRNA) of $\sim 70 \mathrm{bp}$ with a 2 nucleotides (nt) long 3' overhang. The pre-miRNA is transported to the cytoplasm by the Exportin-5:RanGTP complex for further processing. This complex can recognize the 2 nt 3' overhang to make transport possible through nuclear pores. Following the translocation of pre-miRNA through the nuclear pore, pre-miRNA is released into the cytosol (O'Brien et al., 2018; Okada et al., 2009).

The cytoplasmic pre-miRNA is further cleaved at the terminal loop by RNase III endonuclease Dicer, and dsRBD Loquacious (Loqs) or TAR RNA-binding protein (TRBP) in humans, consequently producing the mature 22 nt miRNA duplex (Jiang et al., 2005; Macrae et al., 2006; Saito et al., 2005; Zhang et al., 2004). Pre-miRNA can give rise to abundant maturemiRNAs strands from 5' (left arm) or 3' (right arm). Only one of the mature-miRNA strands 
is loaded into an Argonaute (Ago) protein and facilitates the formation of RNA-induced silencing complex (RISC/miRISC). The choice of the $5 p$ or the $3 p$ miRNA complementary strand loaded to RISC is based partly on thermodynamic stability at 5'end. The strand with the lower stability that is loaded to the RISC is known as the "guide strand", and the unloaded strand is called the "passenger strand," which will eventually get degraded by cellular machinery (Broughton et al., 2016; Guo and Lu, 2010; Meijer et al., 2014). Although much progress has been made understanding their biogenesis and biological functions, the mechanisms allowing miRNAs to silence gene expression in animal cells are still under debate.

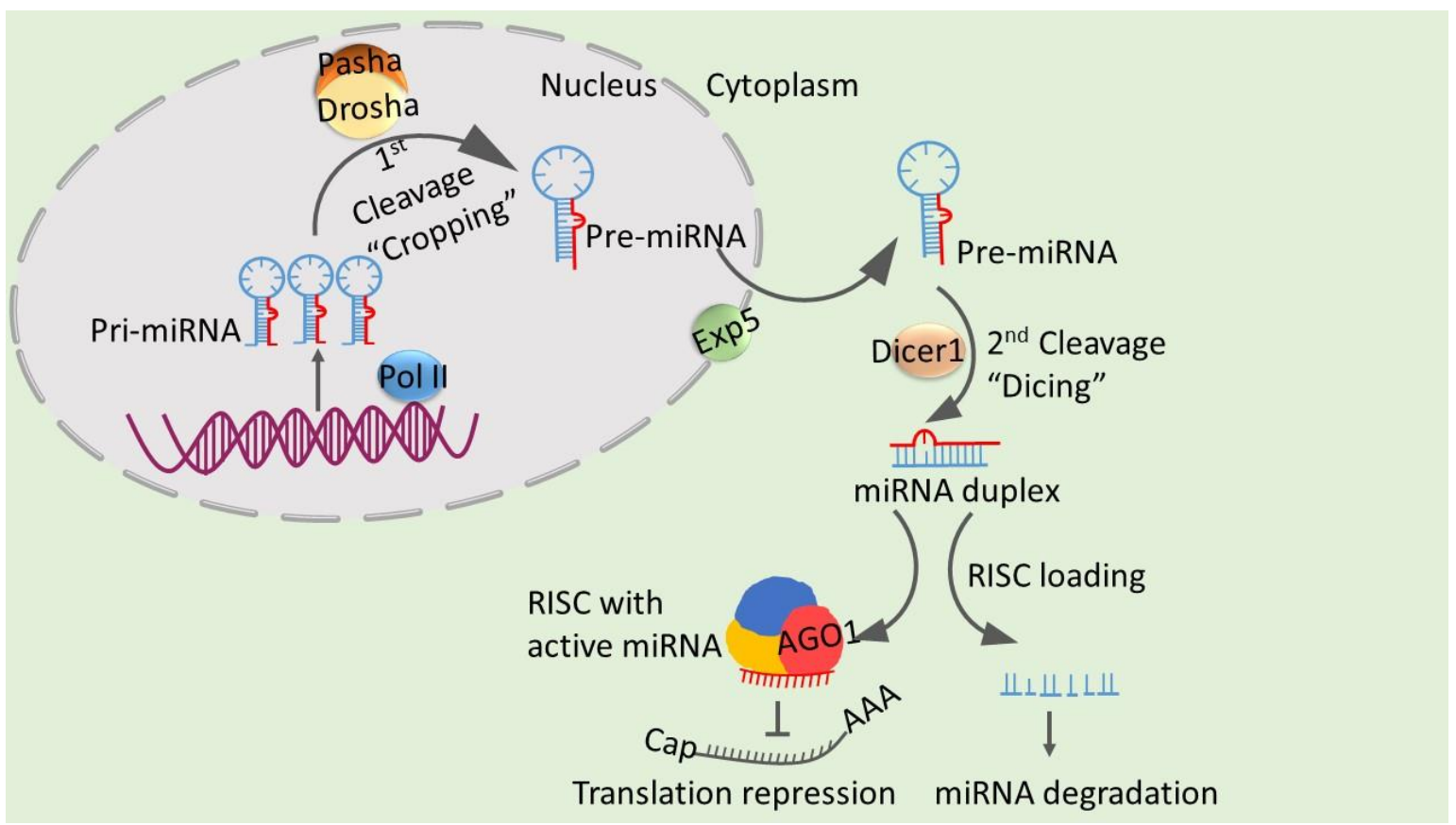

\section{Figure 2. The canonical pathway of miRNA biogenesis}

MiRNA biogenesis starts with the generation of pri-miRNA transcript. The microprocessor complex (Drosha and its binding partner Pasha) cleaves the pri-miRNA to pre-miRNA. The pre-miRNA is then exported to the cytoplasm via Exp5, followed by Dicer1 processing to produce a miRNA duplex. Either the $5 p$ or the $3 p$ strand of the miRNA duplex is then loaded to a complex containing Ago1 to form the miRISC, and the other strand gets degraded. MiRISC can bind to target mRNA to induce transitional inhibition.

\subsubsection{MiRNA mode of action}

MiRNAs can direct the RISC to affect gene expression, mainly via translational repression or mRNA degradation (resulting from mRNA decapping or deadenylation), or a mixture of both. This highly depends on miRNA-target mRNA complementarity. The full miRNA-target mRNA complementarity results in mRNA cleavage in a siRNA-like manner that is mostly observed in plants (Yekta et al., 2004). In a few cases, higher degrees of miRNA- 
mRNA complementary can lead to the destabilization of the miRNAs itself (Ameres et al., 2010; Baccarini et al., 2011; Xie et al., 2012). In contrast, animal miRNAs recognize their target mRNAs through partial complementarity, resulting in recruitment of additional effector proteins, which induce translational repression and/or mRNA decay via deadenylation or decapping (Bartel, 2004; Behm-Ansmant et al., 2006; Wu et al., 2006). Not all miRISC targeted mRNA are destined for degradation. MiRISC and targeted mRNA has been reported to be localized in different cellular compartments, such as rough endoplasmic reticulum, early and late endosomes, multivesicular bodies, as well as in stress granules (SG) to regulate miRISC and mRNA concentration over time to promote efficient gene regulation (Barman and Bhattacharyya, 2015; Bose et al., 2017; Gibbings et al., 2009; Kucherenko and Shcherbata, 2018b).

\subsubsection{MiRNA target identification and seed sequence}

The discovery of the first ever reported miRNA lin-4 also shed some light on the mechanism of miRNA target identification. The clues came from the observation that lin-4 has some sequence complementarity at 3'UTR to its target mRNA lin-14 (Lee et al., 1993; Wightman et al., 1993). Further studies on miRNAs revealed that canonical miRNA-target interactions is based on full complementarity to a 7-8 nt long sequence at 5'region of miRNA, also known as the "seed sequence", together with partial complementarity of the rest of the miRNA to target mRNA (Brennecke et al., 2005; Doench and Sharp, 2004; Kloosterman et al., 2004; Lewis et al., 2003). However, a study in C. elegans has shown that both 5', as well as 3' regions of miRNA, are important for stable and specific miRNA target interaction (Broughton et al., 2016). The discovery of the seed sequence has made it possible to develop target prediction algorithms to generate databases to improve the prediction of target mRNAs for a given miRNA and prediction of regulatory miRNAs for a given mRNA (Enright et al., 2003; Kheradpour et al., 2007; Ruby et al., 2007). Many miRNA families are not conserved between plants and animals. Similarly, poorly conserved are their biogenesis, mode of regulation, as well as cellular localization of miRNA processing, suggesting that miRNAs arose independently in plants and animals (Lee et al., 2003; Zhang et al., 2007). Some miRNAs are highly conserved during evolution (Bushati and Cohen, 2007; Liu et al., 2012; Pasquinelli et al., 2000), but their targeted mRNA can differ between species (Chen and Rajewsky, 2007). Even if the mature miRNA is itself not conserved, its seed sequence is 
evolutionarily conserved, highlighting the relevance of the seed sequence in target recognition (Brennecke et al., 2005; Lewis et al., 2005; Lewis et al., 2003). Though much is known about their biogenesis, mode of action, and complexity, understanding the functions of individual miRNAs still remains challenging.

\subsubsection{MiRNAs role in gene regulation}

Due to their peculiar nature of the small size and numerous possibility of target identification via seed sequences, it is no doubt that one miRNA can target several different mRNAs and each mRNA can be targeted by several different miRNAs, generating a complex network of gene expression and regulation. They are known to canalize gene expression, which is contrary to their paradoxical properties of strongly conserved but with not so similar in function or not so conserved but functionally similar. Though they are known to down-regulate their target mRNA in many cases, downregulation of the target gene has been found at a modest level mostly exceeding not more than 50\% (Baek et al., 2008; Selbach et al., 2008). The latter study also showed that miRNAs can directly repress translation of many genes contributing to the fine-tuning of protein synthesis from various other genes. They often have the dual function of expression tuning and expression buffering of their target genes (Wu et al., 2009). These two mechanisms are somewhat independent and are achieved by feedforward and feedback regulatory loops. Fine-tuning ensures the precise amounts of target gene expression required for biological processes, which cannot be achieved by transcriptional control alone whereas, expression buffering reduces the variance of highly expressed target gene. There are many ways to achieve the expression-tuning and expression-buffering modes. Expression tuning can be achieved by: 1) directly down-regulating the target gene (Cacchiarelli et al., 2011a; Xiao et al., 2007; Yatsenko and Shcherbata, 2014), 2) a coherent feed-forward loop in which two pathways work coherently to ensure the silencing of the target gene (Hornstein et al., 2005; Makeyev and Maniatis, 2008), and 3) a double-negative feedback loop where a miRNA can down-regulate a target gene that is coupled with second gene, and either one of the genes - but not both - will be expressed due to their target miRNA (Li et al., 2006). Similarly, expression buffering can be achieved through: 1) an incoherent feed-forward loop wherein the expression of one gene is dependent on the expression of a second gene directly or indirectly due to the presence of miRNA. The presence of miRNA is directly proportional to the increase in level of the first gene (O'Donnell et al., 2005), 2) a 
negative feedback loop in which both miRNA and its target gene buffer each other's expression (Adams et al., 2018; Martinez et al., 2008; Yatsenko et al., 2014), and 3) an incoherent feedforward loop in which more than one gene can buffer the expression of another gene against the fluctuations in miRNA expression (Choi et al., 2007). Though miRNAs play an important role in buffering and regulating gene expression, they are highly dispensable. Their loss results in very mild or no phenotype in well-controlled laboratory environments (Li and Carthew, 2005; Miska et al., 2007). However, the evolutionary conservation of many miRNAs as well as their functional effectiveness and fast response demonstrate that they are important regulators of spatial and temporal expression patterns of their targeted genes, their downstream targets, and their cofactors.

\subsubsection{MiRNAs as biomarkers}

MiRNA biogenesis is under tight temporal and spatial control, and deregulation in this process is associated with many human diseases. And many of the miRNA implications have been made to diagnostic and therapeutic application in human diseases. The first identified miRNAs in a human-related disease was the polycistronic miRNA cluster miR-17 92. Haploinsufficiency of these miRNAs is responsible for microcephaly, short stature, and digital abnormalities in both humans and mice (de Pontual et al., 2011). The same cluster miRNAs were found to be downstream of an oncogene $(c-M y c)$, and upstream of their target gene $(E 2 F 1)$, which promotes the cell cycle (O'Donnell et al., 2005). The implication of miRNAs in cancer is emerging, and many miRNAs are known to be altered in cancer patients (Koturbash et al., 2011; Munker and Calin, 2011; Tufekci et al., 2014). Downregulation of $m i R-15 a / 16-1$ is associated with multiple myeloma in humans (Li et al., 2015), and $m i R-1$ and $m i R-133 a$ promote prostate cancer by down-regulating purine nucleoside phosphorylase (PNP) (Kojima et al., 2012). Methylation of the miR-137 promoter is also associated with derepression of Cyclin-dependent kinase $6(\mathrm{Cdk} 6)$ causing squamous cell carcinoma of head and neck in humans (Langevin et al., 2011). In humans, loss of miR-137 is also associated with intellectual disability (ID) (Willemsen et al., 2011). MiRNAs are also studied extensively as a biomarker for aging (Dhahbi, 2014). MiR-34a was found to be increased reciprocal to the age of the mouse and was directly proportional to the decrease of its target SIRTI (Li et al., 2011). The same miRNA in Drosophila (miR-34) has been reported to cause aging and neurodegeneration (Liu et al., 2012). Many miRNAs are associated with age-related diseases. MiR-21 is highly expressed in patients with cardiovascular disease (Olivieri et al., 
2012), whereas $m i R-433$ is associated with Parkinson's disease by negatively regulating fibroblast growth factor 20 (FGF20) (Wang et al., 2008).

Many miRNAs also have implication in Muscular Dystrophy. Their expression levels are altered in primary muscular disorders including various kinds of MDs (Eisenberg et al., 2007; Greco et al., 2009). Muscle miRNAs are found to be enriched in the serum of DMD patients; particularly $m i R-1, m i R-133$, and $m i R-206$ have been proposed as diagnostic markers for DMD, as the disease severity correlates with the miRNAs' expression (Cacchiarelli et al., 2011b).

Due to their multiple targeting capacities as well as involvement in multiple biological processes, miRNAs represent promising therapeutic targets, and several pharmaceutical companies are already exploring miRNA in therapeutic development. One such example is the invention by MIRagen Therapeutics of chemically modified structures of miR-15/195 and $m i R-29$ that have reached preclinical development in pathologies of metabolic as well as cardiovascular disease (Shah et al., 2016). The same company has three more miRNAs in their drug discovery pipeline, namely Cobomarsen (MRG-106), an inhibitor of $m i R-155$ for treatment of blood cancer (Phase I, and II clinical trial), a synthetic miRNA mimic of $m i R$ $29 b$ (MRG-201 in Phase II clinical trial), and miR-92 (MRG-110) for pathologic fibrosis and heart failure (www.miragen.com).

\subsubsection{MiRNAs as stress regulators}

Stress can range from prolonged disease conditions to short-term changes in environmental or physiological cellular conditions. To adjust to harsh environments, cells can turn certain pathways on or off to maintain cellular homeostasis. MiRNAs are ideal candidates for stress response regulators as each one can target multiple mRNAs, which can be part of multiple signaling cascades. Under stress conditions, both miRNA and Ago protein are localized to stress granules where mRNAs bound by stalled $40 \mathrm{~S}$ ribosomes accumulate due to stressinduced repression of translation initiation (Leung and Sharp, 2007). Moreover, miRISCs are detected in many membrane-less structures, e.g. ribonucleoprotein (RNP) granules such as stress granules (SG), processing bodies (PBs), GW bodies, and neuronal granules (Kucherenko and Shcherbata, 2018a, b). In flies, miRNAs are known to mediate immediate as well as reversible stress responses to maintain cellular homeostasis (Cicek et al., 2016; Edeleva and Shcherbata, 2013). In many other model organisms, including mice and flies, miRNA knock-outs do not, in most cases, show gross developmental or viability phenotypes, 
but the same miRNA mutants can exhibit noticeable phenotypes under stress conditions (Leung and Sharp, 2007, 2010). All these examples lead to a common notion, that miRNAs are profound agents of stress-response pathways. Recently, miRNAs have been implicated as major stress-response factors in many organisms and are known to contribute to disease relevance (Leung and Sharp, 2010; Mendell and Olson, 2012). Yet, signaling systems connecting stress and changes in miRNA expression patterns remain to be discovered.

\subsection{Stress and Muscular Dystrophies}

MiR-1 has been reported to target Glucose-6-phosphate dehydrogenase (G6PD) to control oxidative stress, and oxidative stress is known to cause progression of DMDs (Cacchiarelli et al., 2010). ER stress is associated with patients with SMA, which is known to cause muscle degeneration similar to MDs ( $\mathrm{Ng}$ et al., 2015). In flies, it was shown that stresses such as high temperature, low-sugar foods (further sugar starvation), oxidative stress, and aging can cause muscle degeneration in wild-type flies, and this phenotype is accelerated in dystrophic flies (Kucherenko et al., 2011). This indicates that both stress and MDs can act via a common pathway, and dystrophic phenotypes can be recapitulated simply by inducing stress. The same study identified many novel interactors of the DGC that are involved in mechanosignaling and cellular stress response, indicating that the DGC may act as a sensor in mechanical stress-response pathways. Since many miRNAs are deregulated in MDs and they have emerged as diagnostic biomarkers (Chapter 1.3.5), miRNAs can be good candidates for common molecular agents between stress and muscular dystrophies. Hence, it is important to understand the molecular circuits of how stress can modulate levels of miRNAs that contribute to disease pathogenesis.

\subsection{MiRNAs profiles in Muscular Dystrophies}

In DMD, the Dys-Syn-nNOS pathway is known to regulate the miRNA expression by Snitrosylation of HDAC2 (Cacchiarelli et al., 2010), and altered miRNAs expression also correlates with the severity of MDs (Chapter 1.3.5). Similarly, miRNAs are also found to regulate the DGC (Cacchiarelli et al., 2011a; De Arcangelis et al., 2010), indicating the important role of miRNAs in balancing the epigenetic network in MDs. In a $m d x$ mouse, an animal model of human DMD, $m i R-1$ and $m i R-133$ are downregulated in differentiating myoblasts in the absence of Dys (Greco et al., 2009; McCarthy and Esser, 2007). miR-1, in particular, is evolutionarily conserved and has been shown to act in a positive feedback loop 
by modulating epigenetic profiles of muscle genes, e.g. Mef 2 and Twist, by targeting HDAC4 (Chen et al., 2006; Sokol and Ambros, 2005). Not only does the DGC regulate miRNAs, but many miRNAs are also found to regulate the DGC. MiR-222 is known to regulate the DysSyn-nNOS pathway by targeting the 3'UTR of $\beta 1$-Syntrophin (De Arcangelis et al., 2010), while $m i R-31$ is known to inactivate the Dys-Syn-nNOS pathway by targeting the 3'UTR of Dys (Cacchiarelli et al., 2011a). MiR-206, which positively regulates muscle differentiation, can target Utrophin, a Dys homolog in flies and, like miR-1/133, is a diagnostic biomarker of MDs (Chapter 1.3.5). All of these studies indicate the important role of miRNAs in MD development. A miRNA microarray screen in $D g$ and Dys mutant flies, as well as hyperthermia in dystrophic and wild-type flies revealed groups of miRNAs that were deregulated under differential stress or dystrophy or in both stress and dystrophic conditions (Marrone et al., 2012). Of 110 miRNAs identified in the screen, $65 \%$ (28 out of 43 ) of the miRNAs that are defined in all functional groups were found to be common to more than one group. This study also reported $m i R-956, m i R-980$, and $m i R-252$ are regulated via the Dg-Dys-Syn1 dependent pathway. The study also showed tissue-specific expression patterns of all three miRNAs that were either in muscle, brain, or both, indicating the diverse roles of the DGC. The study also highlighted important links of miRNA expression profiles to stress, muscular dystrophy, and DGC signaling. In particular, the third category revealed the miRNAs that do not change normally under stress but are upregulated in dystrophic mutants and the miRNAs that are downregulated as a normal stress response but do not change in dystrophic mutants. Since there are similarities in stress and dystrophy, these miRNAs are good candidates to be involved in regulating the DGC signaling in response to stress. Though this implies flies are an excellent model to determine novel factors that can potentially play a role in the pathogenesis of MDs including miRNAs as potential therapeutic targets, much work is needed to fully understand the molecular mechanism of the DGC-dependent miRNAs to monitor the pathological progression of the disease.

\subsection{Architecture of adult Drosophila muscle}

The DGC provides mechanical stabilization of the muscle sarcolemma by anchoring the ECM to the cytoskeleton. It also provides signal transduction platform between the inner and outer membranes of the muscle cell, and providing a scaffold responsible for the membrane localization of signaling proteins, such as Syn and nNOS. nNOS can nitrosylates the HDACs to regulate gene transcription that includes muscle progenitor cells. Muscle degeneration is 
a hallmark of MDs. It can occur due to various reasons, such as physiological and pathological stimuli (e.g., fasting, cachexia) or genetic disorders (e.g. inherited or acquired myopathies). Starvation usually results in muscle atrophy, which is loss of muscle mass due to an increase in protein degradation or a decrease in protein synthesis (Piccirillo et al., 2014). Any prolonged disease state is immense stress to the organismal system to withstand the daily requirement of the fully functional active state. Muscles can withstand laborious and continual mechanical stress, and when damaged they can be repaired by the progeny of satellite cells. In response to disease conditions like MDs, muscle fibers are replaced with fat and fibrotic tissues. In the mammalian model, lack of Dys causes the muscle sarcolemma to deteriorate, leading to damage that cannot be easily repaired via response to muscle satellite cells. This results in chronic inflammation, which eventually results in replacement of the muscle fibers by adipose or fibrotic tissues (Porter et al., 2002). Importantly, similar mechanisms can also be seen in flies.

Just like in humans, adult fly muscles are specialized to perform various specific functions, such as flying, jumping, and walking. Fly muscles share structural and functional similarity with vertebrate muscles. Similar to mammals, flies have both oxidative as well as glycolytic muscles. Both the direct and indirect flight muscles are oxidative muscles in flies, whereas leg muscles are glycolytic muscles in flies (Taylor, 2006). Individual muscle groups are made from the same fiber type, but the fiber types can differ for different muscles that are destined for similar functions (Bryantsev et al., 2012; O'Donnell et al., 1989).

One of the distinct and the largest muscle groups in adult Drosophila is the indirect flight muscle (IFM). The IFMs are oxidative muscles resembling Type I muscles in mammals that are sensitive to nutrient supply as well as loss of muscle stimulus by nerves or NMJ disorders such as ALS. Adult Drosophila has two groups of IFMs, namely DLMs (dorsal-longitudinal muscles) and DVMs (dorsal-ventral muscles) (Figure 3). The IFMs function as a single contractile unit, generating momentum during flight (Dutta et al., 2004). In humans, adult satellite cells allows regeneration of muscle tissue following the injury. Muscle satellite cells are recently discovered in flies, and muscle regeneration upon certain injuries have been reported, there are still more room to explore in muscle regeneration upon genetic disorders in flies (Chaturvedi et al., 2017; Gunage et al., 2017). Fly muscles are also arranged in a stereotyped manner, making them easy to identify and quantify ranges of phenotypes that can be spotted in each individual group. Fly muscles also degenerate in dystrophic as well as in other stress conditions. For this project, DLMs of fly muscles were scored and quantified for muscle degeneration phenotypes. 

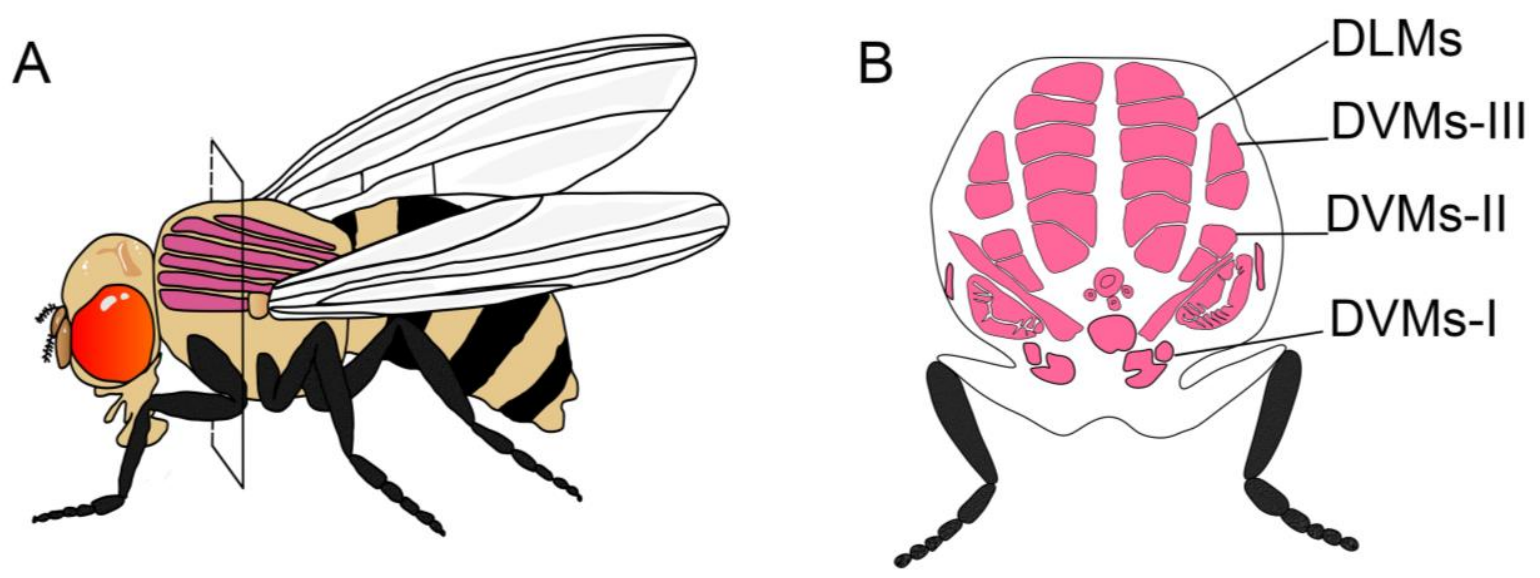

Figure 3. Drosophila Indirect Flight Muscles

(A) Schematic representation of a cross-section of indirect flight muscles (IFMs) of Drosophila melanogaster. (B) IFMs of fly consists of dorsal longitudinal muscles (DLMs) consisting of twelve fibers in total, and three groups of dorsal-ventral muscles (DVM-I/-II/III) in the thorax. These muscles function as a single until during flight.

\subsection{Sterility and Muscular Dystrophy}

Though there is no direct evidence for sterility in MD patients, several DGC components are associated with genes and proteins contributing to infertility in male (Rouillard et al., 2016). Mutation in a gene related to LGMD type 2B in C.elegans (fer-1) is known to cause sterility (Bashir et al., 1998). Similarly, Dg mutant in C.elegans ( $d g n-1)$ are known to be viable, but sterile (Johnson et al., 2006). They show severe disorganization of somatic gonadal epithelium and motor neuron axon guidance defects (Johnson et al., 2006). The DMD-null male mouse is also reported to be sterile (Kudoh et al., 2005). Additionally, some studies indicated that flies lacking POMT expression (required for glycosylation of Dg) are sterile and non-viable at elevated temperatures (Cooley et al., 1988; Ueyama et al., 2010). POMT is a critical enzyme required for glycosylation of $D g$ and is associated with CMD and Walker- Warnurg Syndrome. Studies from flies have shown that glycosylation is a critical step for a mature sperm to fertilize eggs (Perotti et al., 2001). Both Dg and Dys are found to have stage-specific expressions in Sertoli cells in mammals (Zimmermann et al., 2015). Sertoli cells are somatic cells that form the occluding barrier between two somatic cells to provide a unique environment for germline differentiation in each cyst cells. This somagermline barrier is also called the Sertoli cell barrier (SCB) or the blood-testis barrier (BTB) (Cheng and Mruk, 2012; Franca et al., 2012). BTB is a selective permeability barrier maintained by tight junction (in mammals) or septate junction in Drosophila. The BTB separates the early phases of spermatogenesis. Disruption of BTB in vertebrates is reported 
to result in failure in germline differentiation and ultimately leading to sterility (MazaudGuittot et al., 2010; Mok et al., 2012). Similarly, in Drosophila, knockdown of the somaspecific gene (chic) is known to cause sterility due to defective encapsulation (Fairchild et al., 2015). Core septate junction components such as Cora and Nrx-IV are found to localize between the two somatic cells surrounding the germline throughout the spermatogenesis. Knock-down of these components perturbs permeability barrier and gives rise to rudimentary testes (Fairchild et al., 2015). In larval NMJ of Drosophila muscle, core septate junction protein Cora and Nrx-IV have been shown to interact with Dg. Dg and Cora reciprocally control each other's concentration at larval NMJs (Bogdanik et al., 2008; Marrone et al., 2011b). In addition, it has been reported that localization of Nrx-IV is also dependent on Dg in follicular epithelium of fly ovaries (Schneider et al., 2006). Overall, the role of the DGC, in particularly Dg, exceeds beyond the nervous and muscle systems. Therefore, it is interesting to investigate roles of the Dg in spermatogenesis to better understand its broad biological and cellular functions.

\subsubsection{Spermatogenesis in Drosophila melanogaster}

Adult Drosophila has a pair of testes that are coiled tube-like structures producing sperm throughout the male gametogenesis. Spermatogenesis starts at the apical tip of the testis that contains a pool of stem cells of two separate origins, namely germline stem cells (GSCs) and somatic stem cells (CySCs). Both of these cell types reside in a specialized microenvironment called the stem cell niche. Niche provides architecture and signaling regulation for stem cell maintenance and division. It is composed of the hub (cluster of 1012 somatic cells), GSCs (a cluster of approximately 8 germline cells), and the CySCs (the number of which approximately match the number of GSCs) (Demarco et al., 2014; Hardy et al., 1979). Hub is a signaling center for both, GSCs and CySCs. Under homeostatic conditions, both GSCs and CySCs divide asymmetrically producing two cells; one attached to the hub that maintains the stem cell characteristics and the other daughter cell that differentiates to give rise to gonialblast (GB) and somatic cyst cells (CySC). The progeny of GSCs give rise to GB and the progeny of CySCs differentiate to somatic cyst cell. Differentiating GBs are encapsulated with two somatic cells and undergoes four rounds of mitotic and two rounds of meiotic division to produce spermatogonia. Spermatogonia then differentiate into spermatocytes that undergo elongation and maturation and eventually form the mature sperm (Figure 4). Proper encapsulation is required for proper germline 
differentiation and production of functional gametes. Encapsulation of germline cells by somatic cells also establishes a barrier that is essential for correct germline differentiation (Fairchild et al., 2015). The same study also found that the permeability barrier (BTB) in vertebrates is dependent on septate junction proteins such as NrxIV and Cora. Interestingly, in larval Drosophila NMJ, localization of both of these proteins are found to be dependent on Dg (Bogdanik et al., 2008). In fly ovaries, it has been shown that Dg is expressed in both somatic as well as in germline cells (Deng et al., 2003; Yatsenko et al., 2007). Since spermatogenesis is a dynamic developmental process that requires precisely timed transition between several distinct stages, it is important to investigate further the involvement of DGC components and in particular Dg, regulating cellular mechanism relying on Drosophila spermatogenesis. 
A
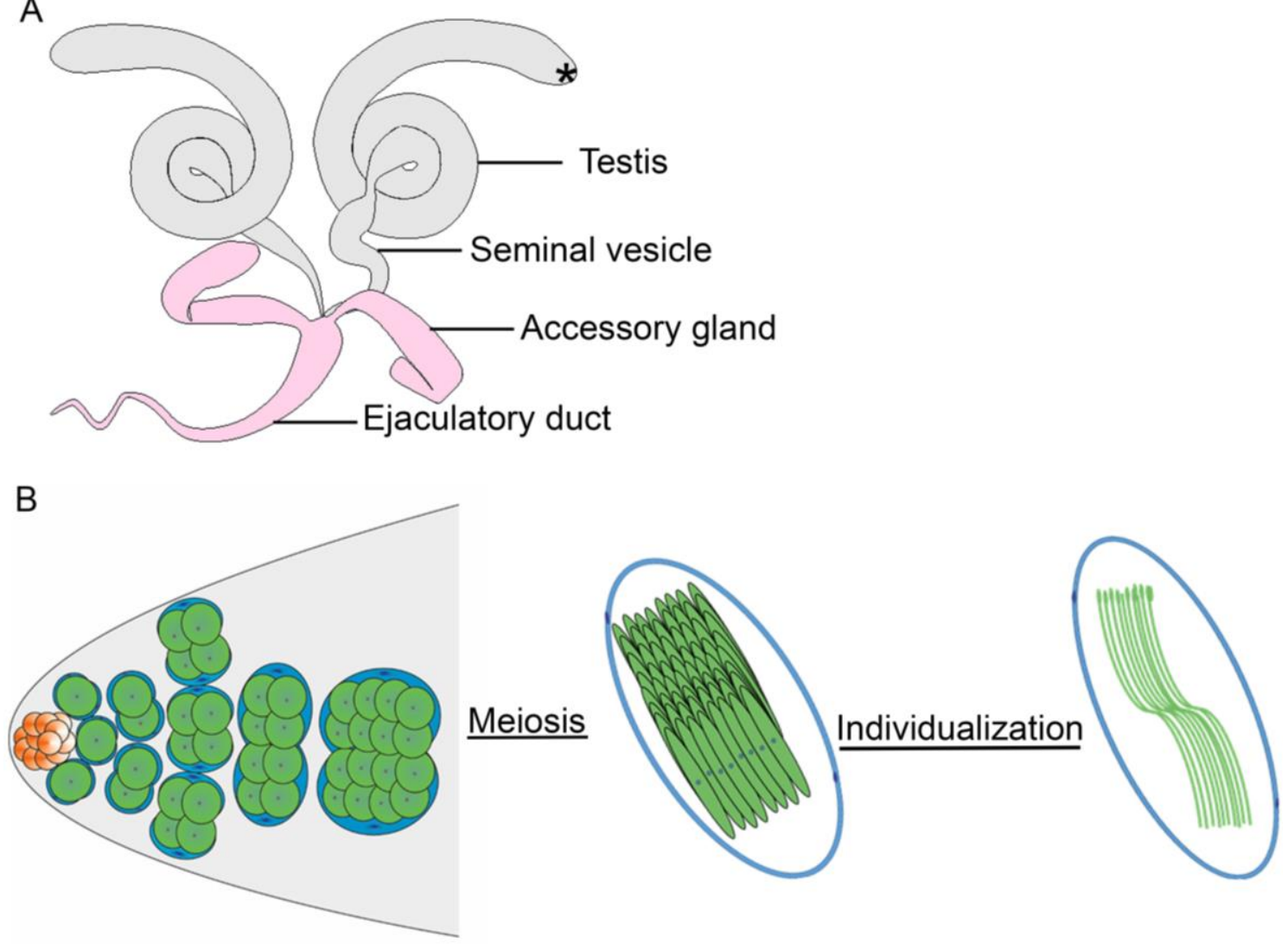

Hub

Gonialblast

\section{Somatic cyst cell}

Spermatocyst

\section{Figure 4. Spermatogenesis in adult Drosophila melanogaster}

(A) Adult Drosophila male gonad consists of a pair of blunt coiled structures called testes, each of which is attached to seminal vesicles and conjoined at a common ejaculatory duct. Spermatogenesis starts at the apical portion of the testes known as a hub and is marked with an asterisk. (B) The apical portion of a testis consists of a hub (marked with asterisk), germline cells (green), and somatic cells (blue). During asymmetric cell division, cells in contact with hub maintain the stem cell characteristics, while the daughter cell differentiates. The differentiating germline cells are called gonialblast, which gets encapsulated with a pair of somatic cells and care called spermatocyst. Within the spermatocyst each gonialblast undergoes 4 rounds of mitotic and two rounds of meiotic division to generate 64 bundles of haploid spermatids. These spermatids go through various steps such as elongation and maturation and finally proceeds to individualization process to make a mature sperm which gets transported to seminal vesicles. 


\subsection{Aims of the study}

The first goal of this project was to identify miRNAs that target the DGC components and influence muscle maintenance. Since most of the MDs' phenotypes can be recapitulated even in wild-type animals under stress conditions, for example, high temperature, starvation and aging, the second goal of this project was to identify miRNAs that act in common pathway shared between stress and MD pathway that controls muscle maintenance and MD development. The final goal of this project was to dissect the role of one identified miRNA targeting DGC components (miR-137), and reveal its biological functions contributing to the DGC regulation and pathogenesis of MDs. 


\section{Materials and Methods}

\subsection{Fly work}

\subsubsection{Fly stocks and maintenance}

Drosophila melanogaster stocks were raised in standard Drosophila food medium with yeast, cornmeal and agar prepared by the fly facility of the MPI for Biophysical Chemistry. All the fly stocks were maintained at well-controlled environment in isolated rooms with a constant humidity of $65 \%$, temperature of $18^{\circ} \mathrm{C}$, and $12-12 \mathrm{~h}$ daily light-dark cycle. All the experimental analyses were carried out on standard Drosophila food media, on standard temperature and humidity condition at $25^{\circ} \mathrm{C}$ or unless otherwise stated. Food vials were replaced with fresh ones every two days throughout the experiments. Food vials used for the experiments were clear plastic vials of small $(28 \mathrm{ml})$, or medium $(68 \mathrm{ml})$ size (Greiner BioOne). All the fly strains used for this project were obtained from the Bloomington Drosophila Stock Center (BDSC) and are summarized in Table 1.

$M i R-137^{k o}$ fly stock, in particular, was backcrossed to 8 generations in the $w^{1118}$ background and was particularly used in analyses made from Chapter 3.2

Table 1. Fly Stock List

\begin{tabular}{|l|l|}
\hline Genotype & BDSC Stock number \\
\hline$w^{1118+}$ & BL 6326 \\
\hline Oregon-R-C & BL 5 \\
\hline Canton-S & Gift from Patrick O'Farrell lab \\
\hline$w^{1118} ; m i R-137^{k o}$ & BL 58893 \\
\hline$w^{1118} ; m i R-252^{k o}$ & BL58901 \\
\hline$w^{1118} ;$ KT40 $\left(m i R-310^{k o}\right)$ & (Tsurudome et al., 2010) \\
\hline$w^{1118}, m i R-927^{K O}$ & BL 58935 \\
\hline$w^{1118} ; m i R-956^{k o}$ & BL 58941 \\
\hline$w^{1118} ; m i R-959-960-961-962^{k o}$ & BL 58944 \\
\hline$w^{1118} ; m i R-966^{k o}$ & BL 58947 \\
\hline$w^{1118}, m i R-975-976-977^{k o}$ & BL 58954 \\
\hline$w^{1118} ; m i R-1000^{k o}$ & BL 58882 \\
\hline$w^{1118} ; m i R-1011^{k o}$ & BL 58887 \\
\hline$w^{1118} ; m i R-137^{D f}$ & BL 8915 \\
\hline
\end{tabular}




\begin{tabular}{|c|c|}
\hline$w^{1118}, m i R-927^{D f}$ & BL 26567 \\
\hline$w^{1118} ; m i R-956^{D f}$ & BL 26579 \\
\hline$w^{1118} ; m i R-966^{D f}$ & BL 9612 \\
\hline$w^{1118} ; m i R-959-960-961-962-963-964^{D f}$ & BL 7724 \\
\hline$w^{1118}, m i R-975-976-977^{D f}$ & BL 23171 \\
\hline$w^{1118} ; U A S-m i r-137$ & BL 59881 \\
\hline$w^{1118} ; U A S-m C h e r r y$.miR-137.sponge & BL 61395 \\
\hline$w^{1118} ; U A S-D s R e d-m i R-927$ & BL 60599 \\
\hline$w^{1118} ; U A S-L U C-m i R-966$ & BL 41211 \\
\hline$w^{1118} ; U A S$-mCherry.scramble.sponge & BL 61501 \\
\hline$w^{1118} ; p U A S t-D g$ & (Deng et al., 2003) \\
\hline$w^{1118} ; U A S-D g^{R N A i}$ & (Deng et al., 2003) \\
\hline$w^{1118} ; \mathrm{Dg}^{1.10 G}$ & Unpublished stock (Shcherbata Lab) \\
\hline$w^{1118}, M h c-G A L 4$ & BL 55132 \\
\hline$w^{1118} ;$ how $^{24 B}-G A L 4$ & BL 1767 \\
\hline$w^{1118} ; t j-G a l 4$ & Kyoto 104055 \\
\hline
\end{tabular}

\subsubsection{Standard Drosophila food media}

$6.25 \mathrm{~g} / \mathrm{l}$ agar (Serva)

$18 \mathrm{~g} / 1$ dry yeast (Saf-Instant)

$80 \mathrm{~g} / 1$ corn flour (Zieler \& Co)

$22 \mathrm{~g} / \mathrm{l}$ beet syrup (Ferdinand Kreutzer Sabamühle $\mathrm{GmbH}$ )

$80 \mathrm{~g} / 1$ malt (Ulmer Spatz)

$0.625 \%$ propionic acid (Merck)

$0.3 \%$ nipagin (Sigma)

Note: The food was cooled down to $55^{\circ} \mathrm{C}$ and then nipagin and propionic acid were added as antifungal and antibacterial agents, respectively.

\subsubsection{Temperature and nutritional stress}

For temperature stress, flies were kept at $33^{\circ} \mathrm{C}, 48 \mathrm{~h}$ post eclosion on standard fly food until desired age. For nutritional stress, i.e. sugar starvation and protein starvation, solid food media was prepared using 1\% agar-agar (Serva) with $0.3 \%$ nipagin (Sigma), and $0.03 \%$ propionic acid (Merck). Flies were raised in this medium $48 \mathrm{~h}$ post eclosion with a small 
quantity of fresh yeast paste prepared from dry yeast and $\mathrm{H}_{2} \mathrm{O}$ up to 5 days. Similarly, for protein starvation, fly food was prepared with (apple juice plate) with 3\% nipagin (Sigma) and $0.03 \%$ propionic acid. Flies were raised in this medium $48 \mathrm{~h}$ post eclosion up to 5 days. Both male and female flies were used for these experiments.

\subsubsection{Aging \& lifespan analysis}

Lifespan experiments were carried out at $25^{\circ} \mathrm{C}$ with 200 male and female flies per genotype. To standardize the age of the experimental flies, parental flies were crossed in plastic cages with apple juice agar plate with fresh yeast paste. After $12 \mathrm{~h}$ of egg-laying time, eggs were washed in PBS and transferred from apple agar plates into falcon tubes. Eggs were allowed to sediment. All the supernatant was removed and $10 \mu \mathrm{l}$ of compact egg/PBS solution was dispensed in medium food vials resulting between 100-150 enclosed flies per vials. Flies were transferred into new food vials after $48 \mathrm{~h}$ of post-eclosion. Both female and male flies were sorted into small food vials separately (10 flies/vial). Flies were tipped onto new food three times a week and deaths were scored at the same time. For statistical analysis, P values were calculated using the log-rank survival test.

\subsection{Genetic screen of miRNAs}

miRNA genetic screen was done by comparing miRNAs loss-of-function along with its overexpression and downregulation using the UAS/Gal4 system in Drosophila (Brand and Perrimon, 1993). Muscle-specific Gal4 lines, such as Mhc-Gal4 and how ${ }^{24 B}$-Gal4 (further how-Gal4) were used to drive UAS-miRNA line to overexpress miRNAs, UAS-miRNAsponge to downregulated miRNAs, and $p U A S t-D g$ was used to overexpress $\mathrm{Dg}$ in fly muscles. Progenies of $w^{1118}$ virgin females crossed to Oregon- $R$ males, and tissue-specificGal4 virgin females crossed to $w^{1118}$ males and used as controls. All the crosses were done at standard Drosophila conditions (chapter 2.1.1), or unless otherwise stated. For muscle analysis, flies at young $\left(7\right.$ day old at $\left.25^{\circ} \mathrm{C}\right)$, aged $\left(30\right.$ day old at $\left.25^{\circ} \mathrm{C}\right)$, temperature stress $(5$ day at $33^{\circ} \mathrm{C}, 48 \mathrm{~h}$ post eclosion), sugar starvation (5 day at sugar starvation, $48 \mathrm{~h}$ post eclosion), and protein starvation (30 day on protein starvation at $25^{\circ} \mathrm{C}$ ) were analyzed as mentioned in Chapter2.3. Similarly, for the analysis of the miRNA function in the somatic cell of Drosophila testes, tj-Gal4 was used as early somatic cell-specific driver line and was crossed to $p U A S t-D g, U A S$-miRNA, or UAS-miRNA-sponge to compare the severity of the phenotypes. 


\subsection{Muscle Analysis}

Adult Drosophila muscle tissues were prepared for analysis by placing the flies into collars and fixing them in Carnoy fixative solution (6:3:1 = Ethanol: Chloroform: Acetic Acid) at $4^{\circ} \mathrm{C}$ overnight. Fly tissues were dehydrated and paraffinized as described by (Kucherenko et al., 2010). Histological sections of $8 \mu \mathrm{m}$ of indirect flight muscles (IFMs) were prepared using Hyrax M25 (Zeiss) microtome and stained with Hematoxylene and Eosin staining. All the chemicals used for this procedure were obtained from Sigma Aldrich. Muscle analysis was done using a light microscope and the frequency of muscle degeneration was quantified as a ratio of degenerated muscles to the total number of muscles that were analyzed per genotype. The analyzed IFM sections were located at the position 200-250 $\mu \mathrm{m}$ from the posterior of the fly thorax.

\subsection{Phenotypic Classification}

\subsubsection{Muscle Degeneration Phenotypes}

The severity of muscle degeneration was categorized into two categories for the simplicity of quantification. Muscles were scored as "strong" muscle degeneration in cases, where all the muscle was deteriorated (absence of fly muscle in the respective area) or substituted with non-muscle tissue. "Moderate" muscle degeneration in cases, where some parts of individual muscle was deteriorated and "mild" muscle degeneration was used as the third category where the muscle showed minor sign of degeneration as punctate-like structures.

Muscle "atrophy" was scored as a separate category for any symptoms of muscles showing loss of muscle integration ranging from the detachment of muscle sarcolemma to low muscle fiber composition.

\subsubsection{Septate junction phenotype}

In elongated spermatids, septate junctions morphology appear as " $\mathrm{H}$ " or "Z" like structure in wild type flies forming the bridge between the two membranes. Both of these shapes were scored as "normal". Any morphological deviation from these shapes, such as a "dot" like structure or an elongated line with no connection to the neighboring cell were scored as "abnormal" septate junction structure. Phenotype was scored beyond $2 / 3^{\text {rd }}$ from the anterior part of the testes were cells enter meiosis and starts to proceeds through the elongation and the differentiation process to form individual sperms. 


\subsection{Gene ontology analysis of predicted miRNA targets}

Conserved Drosophila predicted targets of miRNAs were determined using TargetScan, Release 6.2 (www.targetscan.org). The Generic Gene Ontology (GO) Term Finder tool hosted by the Lewis-Sigler Institute for Integrative Genomics, Princeton University (Boyle et al., 2004) was used to find GO component terms related to predicted targets of each miRNA with a p-value cutoff at 0.01. Visualization was done with the help of Revigo software (Supek et al., 2011) with similarity allowed equal to 0.5

\subsection{Immunohistochemistry}

Fly testes were dissected in cold phosphate buffered saline (PBS/145 mM NaCl, $7.5 \mathrm{mM}$ Na2HPO4, $2.5 \mathrm{mM}$ NaH2PO4 $\mathrm{pH}$ adjusted to 7.4) and fixed using 4\% paraformaldehyde (PFA) for $20 \mathrm{~min}$. Samples were then washed 4 times 15 min each with PBT $\left(0.2 \%\right.$ Triton $^{\mathrm{TM}}$ x-100 (Sigma) in PBS). Fixed tissue was then blocked with PBTB (2 g/l Bovine Serum Albumin (BSA) (AppliChem), 5\% Normal Goat Serum (NGS) (Abcam), and $0.5 \mathrm{~g} / 1$ sodium azide (Sigma)) for $1 \mathrm{~h}$ at room temperature (RT). Primary antibodies (Table 2) were then added and incubated overnight at $4^{\circ} \mathrm{C}$. Samples were washed again the following day 4 times 15 min each with PBT and were blocked in PBTB for $1 \mathrm{~h}$ at RT followed by the addition of secondary antibody solution (Table 2) for 2-3 h at RT. Samples were washed twice in PBT and the procedure was continued with the addition of $10 \mathrm{mg} / 1 \mathrm{DAPI}$ (Sigma) in PBT for 10 min. Samples were washed twice again with PBT and finally, the solution was replaced with mounting medium (70\% glycerol (Sigma), 3\% n-propylgallate (Sigma) in 1x PBS) and left at $4^{\circ} \mathrm{C}$ overnight to equilibrate. Finally, the tissues were mounted on whole slides $(76 \mathrm{X} 26$ $\mathrm{mm}$, Thermo Scientific) and were analyzed with Zeiss LSM700 confocal laser scanning microscope.

Table 2. Antibodies used for immunohistochemistry

\begin{tabular}{|l|l|l|l|}
\hline Antibody & Dilution & Source & Host \\
\hline $\begin{array}{l}\text { anti-Adducin } \\
\text { (Add) }\end{array}$ & $1: 50$ & DSHB & $\begin{array}{l}\text { Mouse } \\
\text { monoclonal }\end{array}$ \\
\hline anti- $\beta$-Gal & $1: 25$ & DSHB & $\begin{array}{l}\text { Mouse } \\
\text { monoclonal }\end{array}$ \\
\hline anti-PH3 & $1: 10000$ & Upstate Biotechnology & Rabbit polyclonal \\
\hline anti-GFP & $1: 5000$ & Abcam & $\begin{array}{l}\text { Chicken } \\
\text { polyclonal }\end{array}$ \\
\hline anti-Vasa & $1: 5000$ & $\begin{array}{l}\text { Gift from Herbert Jäckle } \\
\text { MPI-BPC, Göttingen }\end{array}$ & Rabbit polyclonal \\
\hline
\end{tabular}




\begin{tabular}{|l|l|l|l|}
\hline $\begin{array}{l}\text { anti-Dystroglycan } \\
\text { (Dg) }\end{array}$ & $1: 2000$ & $\begin{array}{l}\text { Gift from Hannele Ruohola-Baker } \\
\text { UW, Seattle }\end{array}$ & Rabbit polyclonal \\
\hline $\begin{array}{l}\text { anti-Armadillo } \\
\text { (Arm) }\end{array}$ & $1: 50$ & DSHB & $\begin{array}{l}\text { Mouse } \\
\text { monoclonal }\end{array}$ \\
\hline $\begin{array}{l}\text { anti-Traffic Jam } \\
(\mathrm{Tj})\end{array}$ & $1: 10000$ & $\begin{array}{l}\text { Gift from Dorothea Godt } \\
\text { UToronto, Toronto }\end{array}$ & $\begin{array}{l}\text { Guinea pig } \\
\text { polyclonal }\end{array}$ \\
\hline $\begin{array}{l}\text { anti-Eyes Absent } \\
\text { (Eya) }\end{array}$ & $1: 50$ & DSHB & $\begin{array}{l}\text { Mouse } \\
\text { monoclonal }\end{array}$ \\
\hline $\begin{array}{l}\text { anti-Disc large } \\
\text { (Dlg) }\end{array}$ & $1: 200$ & DSHB & $\begin{array}{l}\text { Mouse } \\
\text { monoclonal }\end{array}$ \\
\hline $\begin{array}{l}\text { anti-Mega } \\
\text { anti- } \beta 3 \text { Tubulin } \\
(\beta 3 \text { tub) }\end{array}$ & $1: 300$ & $\begin{array}{l}\text { Gift from Reinhard Schuh } \\
\text { MPI-BPC, Göttingen }\end{array}$ & $\begin{array}{l}\text { Mouse } \\
\text { monoclonal }\end{array}$ \\
\hline $\begin{array}{l}\text { anti-Coracle } \\
\text { (Cora) }\end{array}$ & $1: 25$ & $\begin{array}{l}\text { Gift from Renate Renkawitz-Pohl } \\
\text { PUM, Marburg }\end{array}$ & $\begin{array}{l}\text { Guinea pig } \\
\text { polyclonal }\end{array}$ \\
\hline $\begin{array}{l}\text { Alexa } 568 \text { anti- } \\
\text { mouse }\end{array}$ & $1: 500$ & DSHB & $\begin{array}{l}\text { Mouse } \\
\text { monoclonal }\end{array}$ \\
\hline $\begin{array}{l}\text { Alexa } 488 \text { anti- } \\
\text { rabbit }\end{array}$ & $1: 500$ & Invitrogen & Secondary, goat \\
\hline $\begin{array}{l}\text { Alexa } 488 \text { anti- } \\
\text { chicken }\end{array}$ & $1: 500$ & Invitrogen & Secondary, goat \\
\hline $\begin{array}{l}\text { Alexa 568 anti- } \\
\text { guinea pig }\end{array}$ & $1: 500$ & Invitrogen & Secondary, goat \\
\hline
\end{tabular}

\subsubsection{Permeability assay}

Permeability assay was performed as described in (Fairchild et al., 2015). Adult Drosophila testes were dissected in Schneider's Drosophila Medium (Gibco ${ }^{\circledR}$ ) and transferred in medium containing $10 \mathrm{kDa}$ Dextran Dye conjugated to Texas Red ${ }^{\circledR}$ (molecular probes). The final concentration of the dye was $0.2 \mu \mathrm{g} / \mathrm{ml}$. Images were analyzed with Zeiss LSM700 confocal laser scanning microscope.

\subsection{In situ hybridization (ISH)}

Fly tissues were dissected in cold 1x modified Ephrussi-Beadle Ringer's solution (EBR) (for 10XEBR: $1.3 \mathrm{M} \mathrm{NaCl}$ (Merck), $47 \mathrm{mM} \mathrm{KCl}$ (Merck), $19 \mathrm{mM} \mathrm{CaCl}$ (Merck), and $100 \mathrm{mM}$ HEPES (Roth)) and fixed in 4\% PFA in PBS. Fixation time varied depending on the type of tissues (see below). Subsequent procedures were followed as described in (Zimmerman et al., 2013). Flies tissue were then dehydrated and stored at $-20^{\circ} \mathrm{C}$ overnight. Next, tissues were rehydrated and permeabilized for $1 \mathrm{~h}$ in RT with Proteinase K solution $(50 \mu \mathrm{g} / \mathrm{ml}$ Proteinase K (AppliChem) in $50 \mathrm{mM}$ Tris-HCl (VWR) pH 7.5, $50 \mathrm{mM}$ EDTA (Roth)) 
followed by the post-fixation at 4\% PFA in PBT for $30 \mathrm{~min}$ at RT. Primary fixation and permeabilization varied between the tissues (Table 2). Samples were then rinsed and prehybridized in hybridization buffer (50\% formamide (VWR), 25\% $20 \times \mathrm{SSC}, 5 \mathrm{mg} / \mathrm{ml}$ Torula yeast RNA (Sigma), and $0.1 \%$ Tween 20 ) for $1 \mathrm{~h}$ at $60^{\circ} \mathrm{C}$. Hybridization was carried out overnight at $60^{\circ} \mathrm{C}$ with a $40 \mathrm{nM}$ miRCURY LNA probe (Exiqon; dme-miR-137-3p product \# 619638-360) in hybridization buffer. Post-hybridization was done for $1 \mathrm{~h}$ with three subsequent washes of 20 min with hybridization wash solution (no yeast RNA), 50/50 v/v hybridization wash solution/PBT, and PBT at $62^{\circ} \mathrm{C}$. Tissues were then blocked for $1 \mathrm{~h}$ in Western Block (Sigma) and anti-digoxigenin (DIG) conjugated with Alkaline Phosphatase that was diluted 1:2000 in the block and incubated with tissues overnight at $4{ }^{\circ} \mathrm{C}$. Colorimetric detection was done with $10 \mu \mathrm{l} / \mathrm{ml} \mathrm{NBT}$ (Roche) in staining buffer ( $0.05 \mathrm{M}$ Tris pH 9.5, 0.05 M MgCl2, 0.1 M NaCl, 0.1\% Tween 20) for $30-45 \mathrm{~min}$. Samples were washed 3 more times and let to equilibrate in $80 \%$ glycerol in PBS overnight. Finally, tissues were mounted on whole slides (76X26mm, Thermo Scientific), and analysis was done using Zeiss Axiophot microscope.

Table 3. Duration of tissue fixation and permeabilization

\begin{tabular}{|l|l|l|}
\hline Tissue & Primary Fixation & Permeabilization \\
\hline Testes & $1 \mathrm{~h}$ & - \\
\hline Brain & $30 \mathrm{~min}$ & - \\
\hline NMJ & $5 \mathrm{~min}$ & $10 \mathrm{~min}$ \\
\hline
\end{tabular}

\subsubsection{Fluorescence in situ hybridization (FISH)}

All the procedures were followed similar to regular ISH up until blocking of the sample in Western Block (Sigma) for $1 \mathrm{~h}$ at RT. Samples were then incubated overnight at $4^{\circ} \mathrm{C}$ in biotin-conjugated anti-DIG antibody (Jackson Immuno Research) diluted in 1:500 in Western Block. Samples were then washed 6 times for 10 min each and incubated $1 \mathrm{~h}$ in 1:1000 streptavidin-HRP (TSA kit, PerkinElmer) in the block at RT. For nuclear staining, 1 $\mu \mathrm{g} \mathrm{m}^{-1}$ (final concentration) of DAPI was added during the last wash. Tyramide (TSA kit, PerkinElmer) was diluted in 1:50 and added to the sample and incubated overnight at $4{ }^{\circ} \mathrm{C}$ in dark. Samples were washed 3 more times and let to equilibrate in mounting medium (70\% glycerol (Sigma), 3\% n-propylgallate (Sigma) in $1 \mathrm{x}$ PBS) overnight at $4^{\circ} \mathrm{C}$. Finally, tissues were mounted on whole slides (76x26 mm, Thermo Scientific), and analysis was done using Zeiss LSM700 confocal laser scanning microscope. 


\subsection{Genomic DNA extraction from fly leg and the whole fly}

The middle leg was dissected and then transferred in ice-cold PCR tubes containing squishing buffer (10 mM Tris-HCl, $1 \mathrm{mM}$ EDTA, $25 \mathrm{mM} \mathrm{NaCl}$, and $200 \mu \mathrm{g} / \mathrm{ml}$ Proteinase K (AppliChem) with pH adjusted to 8.2). Similarly, the whole fly body was transferred in icecold PCR tubes containing squishing buffer and homogenized using the pestle. Genomic DNA was extracted under the following conditions in a thermocycler (Bio-Rad $\left.\mathrm{T} 100^{\mathrm{TM}}\right)$.

Table 4. Conditions used for genomic DNA extraction

\begin{tabular}{|l|l|l|l|}
\hline \multicolumn{2}{|c|}{ Fly Leg } & \multicolumn{2}{c|}{ Whole Fly } \\
\hline $65^{\circ} \mathrm{C}$ & $60 \mathrm{~min}$ & $37^{\circ} \mathrm{C}$ & $30 \mathrm{~min}$ \\
\hline $95 \mathrm{C}$ & $10 \mathrm{~min}$ & $97 \mathrm{C}$ & $3 \mathrm{~min}$ \\
\hline $4^{\circ} \mathrm{C}$ & until removal & $4^{\circ} \mathrm{C}$ & $\begin{array}{l}15 \text { min high-speed } \\
\text { centrifugation }(13000 \mathrm{rpm})\end{array}$ \\
\hline
\end{tabular}

\subsection{Polymerase chain reaction (PCR)}

For genotyping, PCR from extracted DNA was performed by using HotStart Taq Plus DNA Polymerase (Qiagen). $0.5 \mu \mathrm{M}$ of each primer pair were mixed with HotStarTaq Plus Master Mix (2x), and $150 \mathrm{ng}$ of template DNA with a final volume of to $20 \mu \mathrm{l}$. All the procedures were followed per the manufacturer's instructions. The primer pairs were designed using Primer3: WWW primer tool (http://biotools.umassmed.edu/bioapps/primer3 www.cgi). The amplicons were designed as intron spanning pairs and were ordered from Microsynth unless otherwise stated. Primers are summarized in Table 5.

Table 5. Primers used for $q P C R$

\begin{tabular}{|l|l|l|l|}
\hline Gene & Orientation & Sequence & Purpose \\
\hline$R p l 32$ & Forward & AAGATGACCATCCGCCCAGC & qPCR \\
\hline$R p l 32$ & Reverse & GTCGATACCCTTGGGCTTGC & qPCR \\
\hline$D g$ & Forward & ACTCAAGGACGAGAAGCCGC & qPCR \\
\hline$D g$ & Reverse & ATGGTGGTGGCACATAATCG & qPCR \\
\hline$D y s$ & Forward & GTTGCAGACACTGACCGACG & qPCR \\
\hline Dys & Reverse & CGAGGGCTCTATGTTGGAGC & qPCR \\
\hline Syn_l & Forward & GGCATTGAACCAGACGAGGG & qPCR \\
\hline Syn_l 1 & Reverse & AATCTCAAATACATCGACCC & qPCR \\
\hline $2 S$ & rRNA & TGCTTGGACTACATATGGTTGAGGGTTGTA & qRT- \\
& & & PCR \\
\hline miR-137-3p & $\begin{array}{l}\text { mature- } \\
\text { miRNA }\end{array}$ & UAUUGCUUGAGAAUACACGUAG & qRT- \\
& PCR \\
\hline mini white & Reverse & TTTGTGCGATTGCGGTTTG & PCR \\
\hline miR-137 & Reverse & CCTCAGGCCCGTTTAAATGAGCTGGAA & PCR \\
\hline Gal4 & Forward & GGCTAGAAAGACTGGAACAGCT & PCR \\
\hline Gal4 & Reverse & AGGGCAAGCCATCCGACATG & PCR \\
\hline
\end{tabular}




\begin{tabular}{|l|l|l|l|}
\hline$p U A S T$ & Forward & AGCAACCAAGTAAATCAACTGC & PCR \\
\hline$p U A S T$ & Reverse & TTAAATCTCTGTAGGTAGTTTGTCC & PCR \\
\hline
\end{tabular}

\subsubsection{Agarose gel electrophoresis}

DNA fragments were separated by length by running linear DNA (PCR products) in $1 \%$ and $1.5 \%$ agarose gel (Sigma) in TAE (40 mM Tris-acetate, $1 \mathrm{mM}$ EDTA with $\mathrm{pH}$ adjusted to 8.2). Samples were then mixed with DNA loading dye (6x, New England BioLabs ${ }^{R}$ Inc.) containing bromophenol blue as a visual marker. Gels were post-stained with Midori green advance (Nippon Genetics) and based on the size of the PCR product, Hyper Ladder $50 \mathrm{bp}$ and $100 \mathrm{bp}$ (New England Bio Labs) were used to determine DNA size.

\subsection{RNA extractions and cDNA synthesis}

Total RNA was extracted from the whole body of 5 male flies or 50 testes per genotype by homogenizing in $200 \mu 1$ Trizol reagent (Ambion). Further procedures were followed as per the manufacturer's protocol. Quantification of total RNA concentration was done using the Nano Drop (ND-1000 Spectrophotometer, Peqlab Biotechnologie GmbH). Total cDNA was reverse transcribed using random primers with High Capacity Reverse Transcriptase (Applied Biosystems) with $1.5 \mu \mathrm{g}$ of total RNA template in a $20 \mu 1$ reaction with following conditions at thermocycler (Bio-Rad $\left.\mathrm{T} 100^{\mathrm{TM}}\right)$.

\subsection{Quantitative PCR (qPCR)}

Fast SYBR Green reagents in Step One Plus Real-Time PCR System (Applied Biosystems) was used according to the manufacturer's instructions to perform qPCR. Each reaction was set up with forward and reverse primer of $300 \mathrm{nM}$ concentrations and $100 \mathrm{ng}$ cDNA as template in the $15 \mu 1$ total reaction volume. Amplification was done using StepOne Plus thermocycler (Applied Biosystems). $\mathrm{C}_{\mathrm{T}}$ values were acquired from StepOne Software (Applied Biosystems), and technical replicate average $\mathrm{C}_{\mathrm{T}}$ values of respective genes were normalized to the endogenous control (housekeeping gene, $R p l 32$ ) to achieve $\Delta \mathrm{C}_{\mathrm{T}}$ value. qPCR data were validated using the $\Delta \Delta \mathrm{C}_{\mathrm{T}}$ method that was achieved by subtraction of $\Delta \mathrm{C}_{\mathrm{T}}$ value of each genotype to the control genotype. Relative expression of the gene of interest was calculated using the formula: $2^{-\Delta \Delta C T}$. For statistics, two-tailed Student's t-test was used for the calculation of $p$ values. 


\subsubsection{Quantitative miRNA expression analysis}

TaqMan ${ }^{\circledR}$ microRNA assays (Applied Biosystems) were used to determine miRNA levels. $2 \mathrm{~S}$ rRNA was used as an endogenous control for this procedure. Reverse transcription was done as described in chapter 2.9, and amplification was done using StepOne Plus thermocycler (Applied Biosystems). Subsequent procedures were carried out as per the manufacturer's protocol. The assay involved reverse transcribing the mature miRNA of interest in a reaction and subsequently detecting the quantity via PCR coupled with fluorescence-labeled oligonucleotide probes.10 ng of total RNA was used for $20 \mu \mathrm{l}$ reaction volume, and $1.33 \mu \mathrm{l}$ of resulting reaction was loaded for the qPCR. The amplicons were ordered from Thermo Fisher Scientific and are summarized in Table.5. The calculation of the relative miRNA expression levels and statistics were done using the respective $\mathrm{C}_{\mathrm{T}}$ values as described in chapter 2.10 .

\subsection{Transfection of Drosophila cell lines (S2R+ cell lines)}

Drosophila S2R+ cells (DGRC) were cultivated in Schneider's Drosophila medium (Gibcoß) with 10\% heat-inactivated fetal bovine serum (FBS, GE healthcare) and 100 units $/ \mathrm{ml}$ penicillin and $100 \mu \mathrm{g} / \mathrm{ml}$ streptomycin (Gibco ${ }^{\circledR}$ ) in $25 \mathrm{~cm}^{2}$ culture flasks in the 6 $\mathrm{ml}$ medium at $25^{\circ} \mathrm{C}$. Cells were split into 96 wells plate (polystyrene black plate, costar) and incubated overnight to achieve confluency of $60-70 \%$. Transfection mixes were prepared using Effectene ${ }^{\circledR}$ transfection reagent (Qiagen) as per manufacturer's protocol. Following amounts of the reporter and constructs were added on the transfection mixes: 50 ng of empty psiCHECK ${ }^{T M}-2$ or $50 \mathrm{ng}$ of psiCHECK ${ }^{T M}-2-D g-3$ 'UTR plasmid fragments (Yatsenko et al., 2014) containing miRNA binding sites, $25 \mathrm{ng}$ of act-Gal4, and 50ng of the $p U A S T$-miRNA plasmid (Gift from Eric Lai). Transfection mix without reporter constructs was used as background control. 


\subsection{Luciferase reporter assay}

To measure firefly and renilla luciferase activity, the Dual-Glo® luciferase assay kit (Promega) was used approximately $72 \mathrm{~h}$ of post-transfection. All the procedures were followed as per the manufacturer's instructions. Luciferase activity was measured as luminescence using Wallac 1420 luminometer (PerkinElmer). To calculate relative downregulation of reporter luciferase values, signals by miRNAs, raw readouts of Renilla to Firefly luciferase values were measured. Next, background values (transfection with empty psiCHECK ${ }^{T M}-2$ with no reporter construct) were subtracted from the respective values. Obtained values were then normalized to empty psiCHECK ${ }^{T M}-2$ to the values of psiCHECK $K_{-2}^{T M}-D g-3$ 'UTR in presence of miRNAs.

All transfections were done in triplicates. Data observed from triplicates were used to determine an average downregulation and standard deviation of the data. Student's twotailed t-test was used for the statistical analysis.

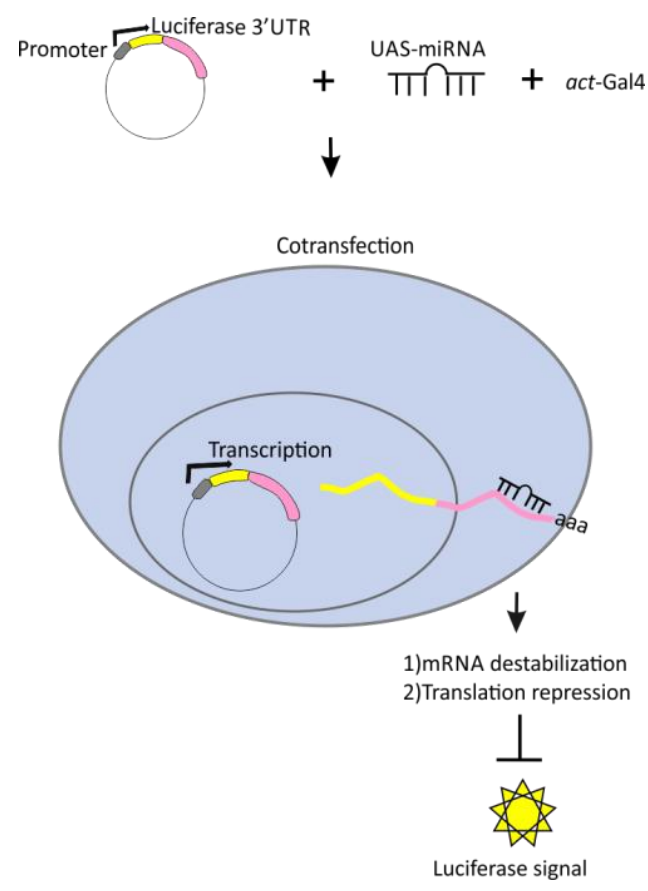

Figure 5. Schematic representation of luciferase assay

$\mathrm{S} 2 \mathrm{R}+$ cells were transfected with $\mathrm{psiCHECK}^{T M}-2$ with or without $D g$-3'UTR fragments together with act-Gal4, as well as, $p U A S T$-miRNA plasmids. When all three constructs are transfected, miRNA will bind to the $D g$-3'UTR resulting in mRNA destabilization or translation repression of reporter gene can be detected as no to less luciferase signal. $p U A S T$ miRNA plasmids transfected with $p s i C H E C K^{T M}-2-D g-3$ 'UTR that doesn't have predicted binding site, or with empty psiCHECK ${ }^{T M}-2$ will have no effect on transcription of the luciferase reporter. Hence, resulting in normal or high luciferase signal as compared to the miRNA inhibition. 


\subsection{Bacterial transformation}

Respective $p s i C H E C K^{T M}-2$ vectors together with $p U A S T$-miRNA plasmids were transformed into chemically competent bacteria (DH5 $\alpha$ Escherichia coli cells, Invitrogen) following the manufacturer's protocol. Cells were heat shocked at $42^{\circ} \mathrm{C}$ for $45 \mathrm{sec}$ followed by incubation on ice for $2 \mathrm{~min}$. Transformed bacteria were then incubated in SOC medium at $37^{\circ} \mathrm{C}$ for $1 \mathrm{~h}$ with a slow shake of $100 \mathrm{rpm}$ in an incubator shaker (Infors AG) and plated on Lysogeny Broth (LB, Invitrogen) agar plate containing $100 \mu \mathrm{g} / \mathrm{ml}$ ampicillin. Plates were incubated overnight at $37^{\circ} \mathrm{C}$. The following day, a single colony was picked using sterile pipette tip and dropped into liquid LB medium with ampicillin at $250 \mathrm{rpm}$ in an incubator shaker (Infors AG) for overnight culture, and were subjected for midi-preparation the next day.

\subsection{Midi-preparation of plasmid DNA}

Bacterial cultures were harvested by centrifuging at $6000 \mathrm{x} g$ for $15 \mathrm{~min}$ at $4{ }^{\circ} \mathrm{C}$. To obtain high purity DNA, Plasmid Plus Midi Kit (Qiagen) was used according to the manufacturer's protocol. The purified DNA was eluted in $200 \mu \mathrm{l}$ of EB buffer (Plasmid Plus Midi Kit). For quality control, total DNA concentration was measured using the Nano Drop (ND-1000 Spectrophotometer, Peqlab Biotechnologie GmbH).

\subsection{Image processing and quantification}

Somatic cell quantification and digital processing of all the microscope images were done using ImageJ-win64 or Adobe Photoshop. Schematic illustrations were done using CorelDRAW X6, and Microsoft PowerPoint. All the heatmaps were drawn using R program version 3.2.3.

\subsection{Bioinformatical analyses}

To find human homologs of the proteins, STRING v10 database (Szklarczyk et al., 2015) with a medium confidence score (0.4) and prediction methods that included neighborhood, gene fusion, co-occurrence, co-expression, experiments, databases, and text mining were used. To assign molecular function and involvement in biological processes of genes, FB2018_05 release FlyBase was applied. To search for the human disease association the http://www.flyrnai.org (Hu et al., 2011) and http://www.genecards.org were used. 


\section{Results}

\subsection{Screen of miRNAs that are predicted to target the DGC}

\subsubsection{The DGC components are predicted to be targeted by multiple miRNAs}

A single gene can be targeted by multiple miRNAs, at the same time, a single miRNA can target multiple genes. Such genes can be positive or negative regulators of the same pathways. To investigate miRNAs that can potentially play a role in MD pathogenesis by targeting the DGC components, we selected miRNAs based on whether or not they are predicted to target the core DGC components.

In Drosophila model of MD, Dg have been shown to be localized postsynaptically at the $\mathrm{NMJ}$ and are required for appropriate homeostatic control of neurotransmitter release and proper localization of Cora and Nrx-IV (Bogdanik et al., 2008). It has also been reported that in the absence of $\mathrm{Dg}$, GluR are improperly localized causing insufficient muscle response (Marrone et al., 2011b). Importantly, mutation in any of these components are also associated with phenotypes similar to MD. Therefore, core components of the DGC as well as the proteins known to interact with Dg were taken together to extract miRNAs that are predicted to target the extended DGC components. Such components consists of multiple protein, among which are Dg, Dys, Syn1/2, nNOS, Scg, Nrx-IV, Cora, Yki, GluR, Lan A/B, Arm, Hts, and Mei-P26 (Chapter 1.1, Figure 1). Several target-prediction tools were utilized to find miRNAs that can target the DGC, namely TargetScan, Release 6.2 (www.targetscan.org), miRBase, Release 20 (www.mirbase.org), and Diana Tools (http://diana.imis.athena-innovation.gr/DianaTools/index.php). This search yielded a group of 72 miRNAs, both conserved and non-conserved, that are predicted to target the transcripts of DGC components (Figure 6).

Interestingly, among these miRNAs, around 20\% (17 out of 72 ) are known to be DGC- or stress-dependent (Marrone et al., 2012), indicating that they can play an integral role in stress response and in MD pathogenesis. Many of these miRNAs can target more than one component of the DGC; however, we were not able to alienate a single group of miRNAs targeting common components of the DGC. Previously it has been shown that miRNAs often regulate multiple components of the same signaling pathway to assure quick and robust response to changes in external conditions and in internal cellular environment (Cicek et al., 
2016; Yatsenko and Shcherbata, 2014). Since Dg is an integral membrane receptor linking the ECM to the actin-based cytoskeleton and is required for correct localization of the majority of the DGC components, we decided to study miRNAs that can target $D g$ and any three other components of the complex. As a result, a much narrower miRNA group was formed which possibly would ensure the dynamic, efficient, and fast control over the DGC pathway activity (Figure 7A). 


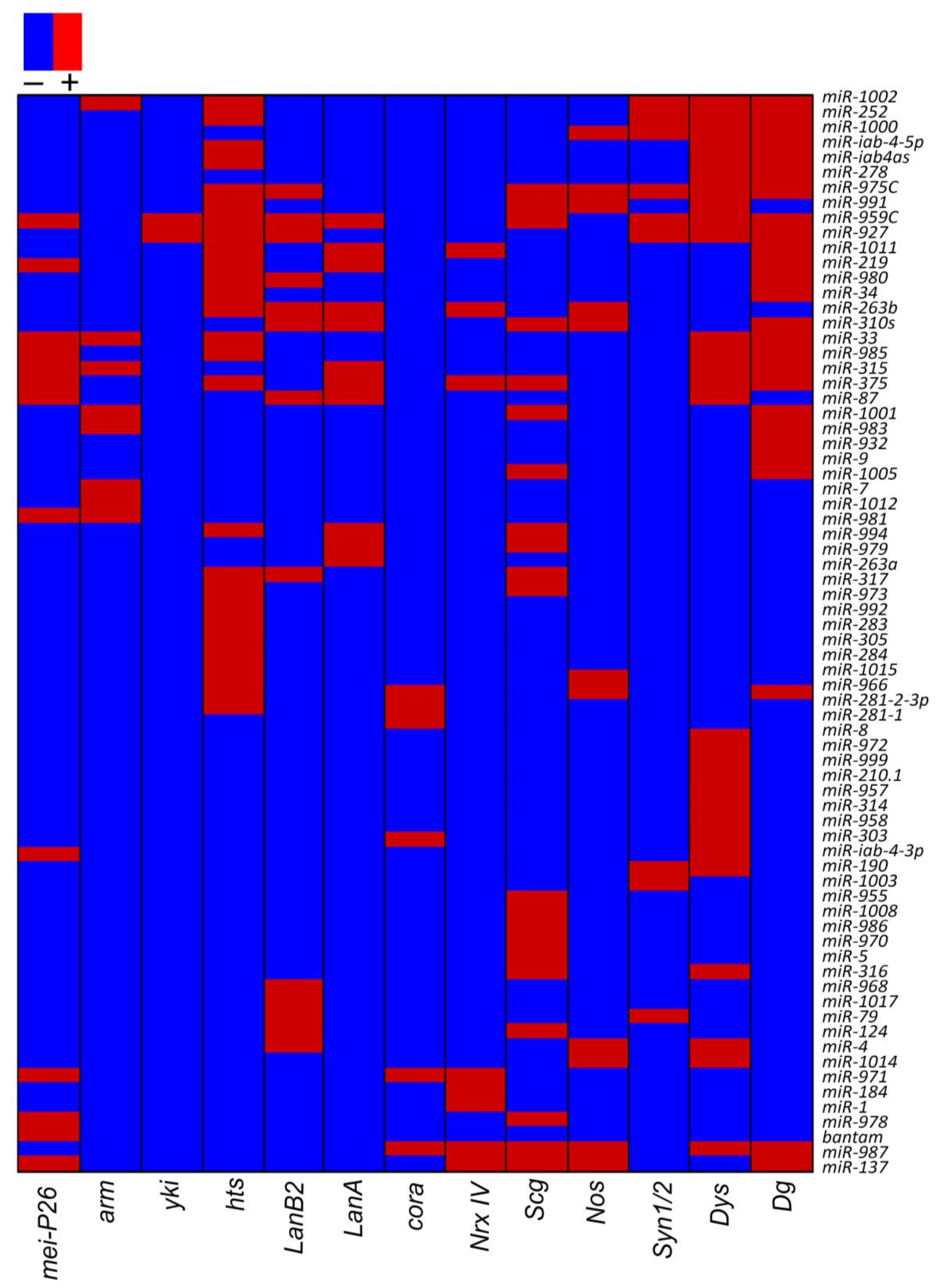

\section{Figure 6. Multiple miRNAs are predicted to target the DGC components}

MiRNAs that are predicted to target any component of the DGC, identified using the online tools TargetScan, miRBase, and DIANA/microT-CDS, are grouped in the heatmap based on their ability to target DGC components. Red indicates a predicted target and blue indicates no target. In total, 72 miRNAs are predicted to target any one of the DGC components. 


\subsubsection{MiRNA mutants have deregulated mRNA levels of $D g, D y s$, and Syn1}

To validate that the miRNAs from this group (Figure 7A) can indeed be involved in regulation of the DGC complex, mRNA levels of the major DGC components were tested in some miRNA mutants. Since the Dg-Dys-Syn1 signaling pathway has been shown to be regulated by miRNAs (Marrone et al., 2012; Yatsenko et al., 2014), we tested the mRNA levels of these three major DGC components via qRT-PCR in ten miRNA mutants. These miRNAs were selected based on the availability of the loss-of-function mutants at the time of the screen and are highlighted with black arrow (Figure 7A). RNA was extracted from the whole body of 1 -week old male flies and were normalized to control. MiR-956 is not predicted to target the DGC and therefore, was used as a negative control. Most of the miRNA mutants revealed deregulated mRNA levels of the DGC components. Since this group of miRNA has been selected firstly, by their ability to target $D g$, we analyzed $D g$ levels in these mutants. MiR-137, miR-252, miR-959C, miR-966, and miR-1000 had Dg mRNA levels upregulated by more than three folds, suggesting that $D g$ can be their direct target. Some miRNAs such as miR-966 showed upregulated mRNA levels of all three components with approximately 2.5-5 fold increase relative to the control. In addition to $D g$ and Dys levels were also upregulated in most of these mutants. Such miRNAs were miR137, $m i R-252, m i R-310 s$, and miR-966 even though, Dys was predicted to be a direct target only for $m i R-252$, the increase in Dys level in these miRNA mutants can be explained because it has been previously shown that Dys stability depends on the levels of $D g$ (Bogdanik et al., 2008; Marrone et al., 2012). Since miRNA mutants have Dg levels increased possibly due to direct targeting of $D g, D y s$ levels would be upregulated. Analysis of $m i R-310 \mathrm{~s}$ mutants showed approximately 2.5 fold increase in mRNA levels of Syn 1 relative to control, suggesting that $m i R-310 s$ can target Synl as predicted (Figure 7B). Though $m i R-927$ and $m i R-975 C$ are predicted to target all three components, the loss of $m i R$ 927 and miR-975C had no influence in the expression levels of Dg, Dys, or Syn 1 . Intriguingly, some of the miRNA mutants had deregulated levels of the DGC components that they are not predicted to target. Since DGC components are known to have compensatory effects on each other levels of expressions, the more rigorous screen is required to find out the miRNAs that could directly target DGC components or indirectly via targeting other factors, leading to MD development. This preliminary screen identified 
miRNAs that have abnormal expression levels of major DGC components and could be involved in maintenance of muscle health via regulation of the DGC.
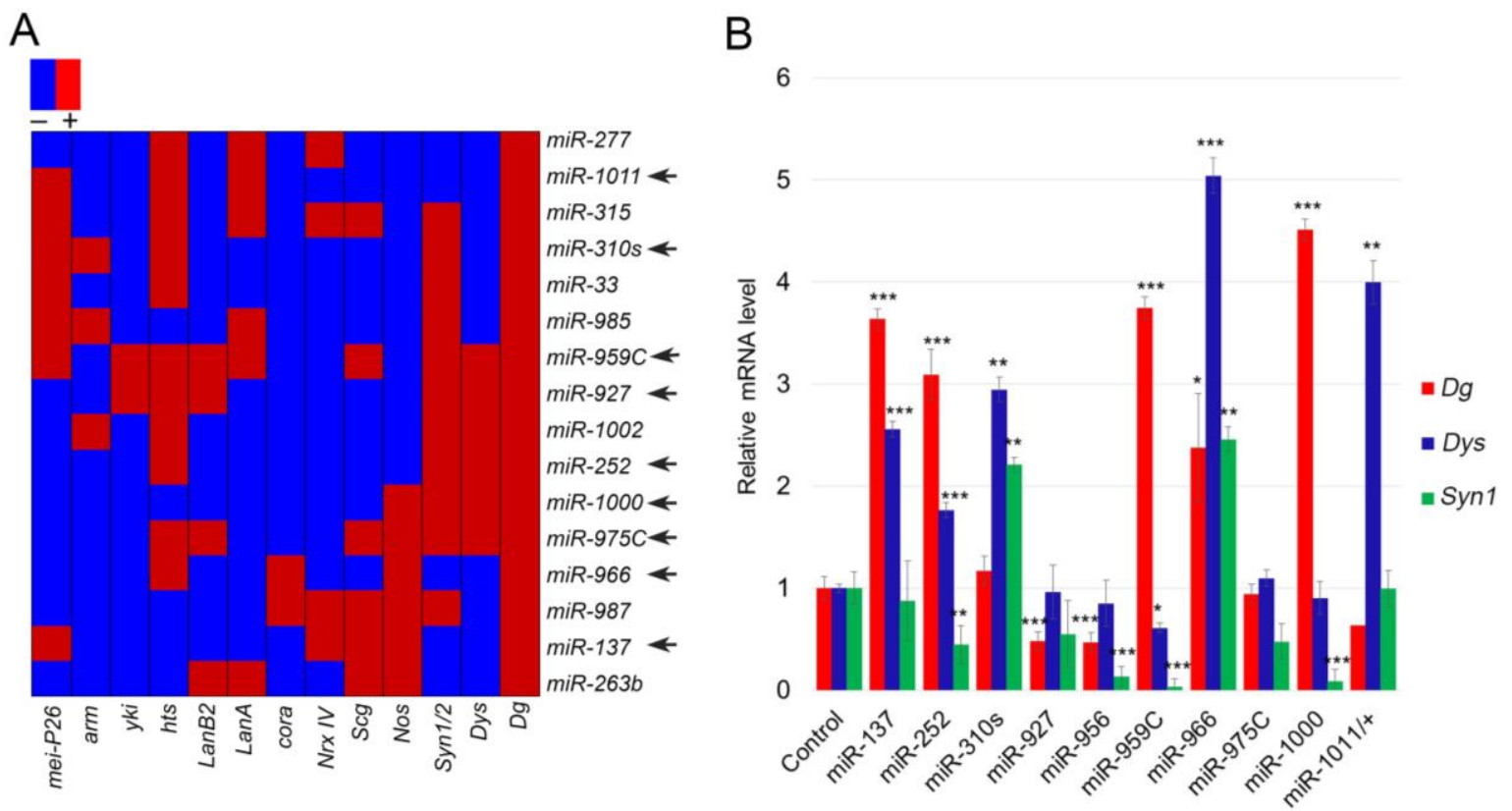

Figure 7. Candidate miRNA mutants have deregulated mRNA levels of the DGC components

(A) Clustering of miRNAs targeting $D g$ as well as any three other components of the DGC results in 16 different miRNAs. Red indicates predicted target and blue indicates no target. The black arrow indicates the availability of loss-of-function miRNA mutants at the time of the screen. (B) qRT- PCR performed on 1-week old male flies revealed altered mRNA levels of $D g, D y s$, and $S y n 1$ shown in red, blue and green bars, respectively. Relative mRNA levels of miRNA mutant flies were normalized to the control flies (Canton-S/OR). MiR-137 and $m i R-252$ mutants have an increase in mRNA levels of $D g$ and $D y s$, while $m i R-310 s$ mutants have an increase in mRNA levels of Dys and Synl. Loss of miR-959C and miR-1000 influence $D g$ level only and miR-966 mutants show an increase in mRNA levels of all three components of the DGC. Error bars represent $\mathrm{AVE} \pm \mathrm{SD}$, and statistical significance was determined by two-tailed Student's t-test $(* \mathrm{P}<0.05, * * \mathrm{P}<0.01, * * * \mathrm{P}<0.001)$. See also Supplementary Table 1.

\subsubsection{Loss of miRNA causes muscle degeneration phenotypes}

Among ten candidate miRNAs, the expression levels of six miRNAs (miR-137, $m i R-252$, $m i R-310 s$, $m i R-959 C$, $m i R-927$, and $m i R-975 C$ ) have been shown to be downregulated in hyperthermia-stressed wild type and/or in dystrophic flies. To study their potential roles in MD development, we decided to test whether the loss of these miRNAs can affect muscle architecture in normal and in stress conditions. We analyzed muscle integrity in miRNA mutants kept at the following conditions: young ( 7 day old at $25^{\circ} \mathrm{C}$ ), aged (30 day old at $25^{\circ} \mathrm{C}$ ), temperature stress $\left(5\right.$ day at $\left.33^{\circ} \mathrm{C}\right)$, sugar starvation $\left(5\right.$ day at $\left.25^{\circ} \mathrm{C}\right)$, and protein 
starvation $\left(30\right.$ day at $\left.25^{\circ} \mathrm{C}\right)$. As expected, most of the miRNA mutants showed progressive muscle degeneration phenotypes (Figure 8). Muscle degeneration phenotypes were quantified as mild, moderate, and strong degeneration as shown in quantification panel (Figure 8A). miRNA mutants, such as $m i R-959 C$ and miR-1000 showed an increase in muscle degeneration phenotypes as early as in young age (approximately $15 \%$ and $17.5 \%$ of muscle degeneration as compared to $5 \%$ in control flies), indicating these miRNAs can be already involved in muscle formation during developmental stages. Most miRNA mutants, such as $m i R-137, m i R-310 s, m i R-959 C$, $m i R-966$, and $m i R-975$ were sensitive to more than one type of stress. Interestingly, only miR-137 mutants were responsive to all stress conditions with more than five times increase in the frequency of muscle degeneration at temperature stress, sugar starvation, protein starvation, and aging, when compared to the young non-stressed animals (Figure 8B). We also noticed that wild type flies itself were sensitive to stress and aging, as they showed stress- and age-dependent muscle degeneration compared to the young age, albeit at the lower than miRNA mutants frequency. Stress- and age-dependent muscle degeneration in wild type flies have also been reported before (Kucherenko et al., 2011). MiR-927 and miR-959C mutants were more responsive to protein starvation with an average muscle degeneration of $20-30 \%$ compared to $4-15 \%$ in young flies of the same genotype. A similar case was observed for miR-966 with $15 \%$ of muscle degeneration compared to $4 \%$ in young flies.

MiR-959C and miR-975C though had stress-dependent muscle degeneration, these mutants were dropped to follow up further, since these miRNAs are in a complex of more than three different miRNAs, each with unique seed sequence and to follow up these miRNAs, an individual miRNA mutants must be generated. MiR-1000 and miR-1011 were not followed up due to the low survival rate, indicating that their role is broader than just muscle maintenance in animals.

Interestingly, loss of muscle degeneration phenotype during stress or aging was also noted in some miRNA mutants when compared to them at young age. Such miRNAs were miR310s, $m i R-966, m i R-975 C, m i R-1000$, and $m i R-1011$. This can be due to the fact that animals with severe phenotype could be dying and the animals that survived would have normal muscles. Contrarily, the expression of these miRNAs could have a negative effect on muscle maintenance and their loss could have a protective role, which would be interesting to study in greater detail in the future. Based on the severity of the muscle phenotypes and stress response, only $m i R-137$, $m i R-927$, and $m i R-966$ were followed further to dissect their roles in MDs and DGC signaling. 


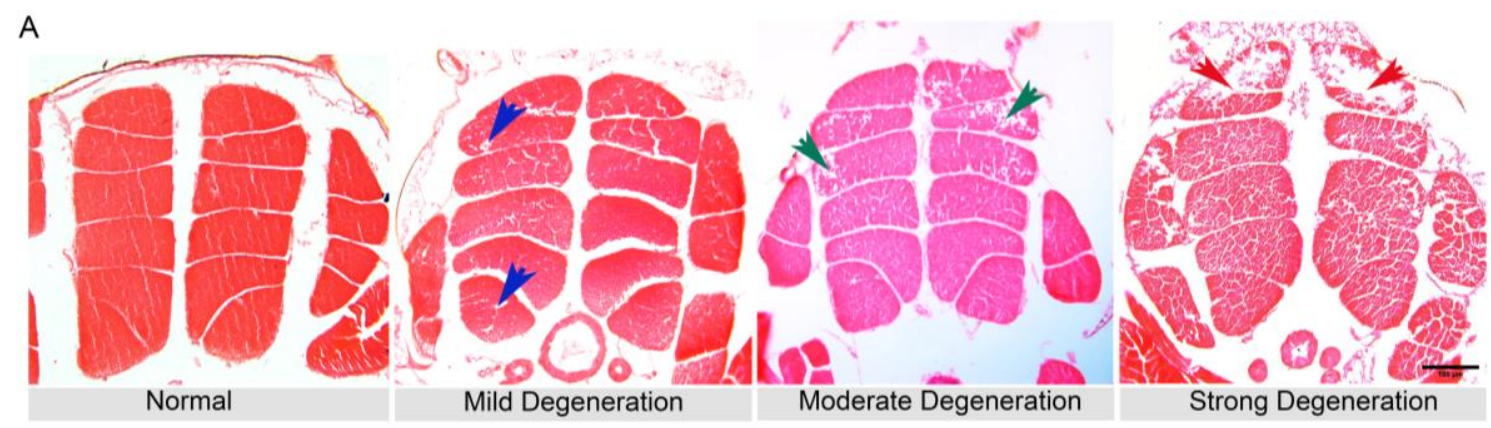

B Percentage of Muscle Degeneration

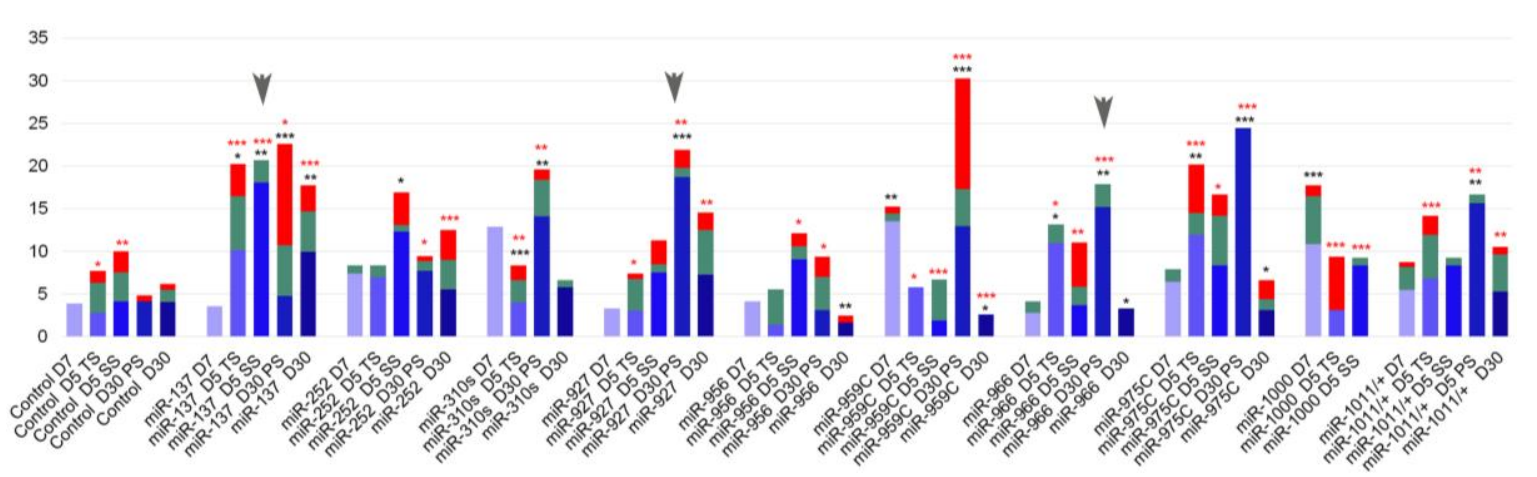

Figure 8. MiRNA mutants show muscle degeneration phenotype that is enhanced upon stress

(A) Representative images of muscle degeneration phenotype in IFMs of adult Drosophila. Blue, green and red arrows show mild, moderate and strong muscle degeneration. (B) A bar graph showing muscle degeneration due to miRNA loss at young, temperature stress, sugar starvation, protein starvation, and aging. Muscle degeneration mainly starts as early as in young flies and the phenotypes are accelerated upon stress even in control flies (Canton$S / O R$ ). $M i R-137, m i R-927$, and $m i R-966$ were responsive to most stress conditions (grey arrows on bar graph). MiR-1000 mutants have the least tolerance for the temperature stress and sugar starvation and had a low survival rate in general, while miR-959C responded only to the protein starvation. Statistical significance was determined by $\chi^{2}$ test with Yate's correction $(* \mathrm{P}<0.05, * * \mathrm{P}<0.01, * * * \mathrm{P}<0.001$ when compared to control flies at same condition), $(* \mathrm{P}<0.05, * * \mathrm{P}<0.01, * * * \mathrm{P}<0.001$ when compared to the same genotype at a young age). Protein starvation was compared to the aged flies. D7= young, 7 day old, TS= temperature stress, $\mathrm{SS}=$ sugar starvation, $\mathrm{PS}=$ protein starvation, and $\mathrm{D} 30=$ aging, 30 day old. Scale bar $100 \mu \mathrm{m}$. See also Supplementary Table 3.

\subsection{Validation of muscle degeneration due to miRNA loss}

The progression of muscle degeneration phenotypes upon stress, due to miRNA loss was further followed up in greater detail for three miRNAs ( $m i R-137, m i R-927$, and $m i R-966$ ). The experiment was done in three independent biological replicates and the appearance of MD phenotypes was quantified in miRNA mutants as mild and strong muscle degeneration (Figure 9A). 
Additionally, muscle atrophy was scored in these animals at normal and different stress conditions. Muscle atrophy, in general, is described as a loss of muscle mass that is driven by an increase in protein degradation or a decrease in protein synthesis. It is an integral feature of systemic diseases including cancer, cachexia, cardiac failure, AIDS, and sepsis. Loss of muscle mass due to aging, also known as sarcopenia is often associated with muscle disuse, fasting (starvation), extrinsic changes in innervation, stem cell function, and endocrine regulation of muscle homeostasis (Demontis et al., 2013). Since most miRNA mutants were responsive to various stresses, including aging, muscle atrophy was also studied in miRNA mutants to dissect miRNA involvement in muscle maintenance.

The frequency of muscle degeneration and muscle atrophy in miRNA mutants kept at normal and different stress conditions, such as young, temperature stress, sugar starvation, and aging were normalized to the frequency of muscle degeneration and atrophy of young control animals. Muscle degeneration phenotypes were scored as mild and strong muscle degeneration as shown with blue and red arrows, respectively and muscle atrophy was scored as shown in green arrows (Figure 9A). As observed before, all animals, miRNA mutants and control were sensitive to stress and develop muscle degeneration and muscle atrophy in response to unfavorable conditions. However, the severity of stress-dependent muscle maintenance defects was different in different mutants. Interestingly, even change in genetic background in controls resulted in different frequencies of muscle degeneration, implying that in general, the muscle tissue is extremely sensitive to stress. For example in Figure 8, heterozygous Canton-S/OR were used as control and the frequency of muscle degeneration was never higher than $10 \%$, while in this experiment (Figure 9), we used $w^{1118}$ mutants crossed to wildtype $O R$ as control. This resulted in the dramatic increase in muscle degeneration phenotypes. A similar observation was made on miR-137 mutants that were back-crossed in the $w^{1118}$ genetic background for 8 generations.

Upon stress and aging, controls themselves showed approximately 1.5-2.5 fold increase in the incidence of muscle degeneration relative to young non-stressed animals. MiR-137 mutants had approximately 2 fold increase in muscle degeneration already at young age when compared to control flies of the same age. This phenotype was even more enhanced upon temperature stress and aging. Similarly, miR-966 mutants showed muscle degeneration phenotypes as early as in young age (1.5 fold higher than control) and the phenotype was enhanced upon aging (3.5 fold compared to 2.5 fold in control). However, $m i R-927$ and $m i R$ 966 mutants did not show temperature stress or sugar starvation response (Supplementary 
Table 4). MiR-927 mutants were responsive only to aging with approximately 4 fold increase in muscle degeneration compared to control flies (Figure 9B).

A comparison of muscle atrophy showed that upon various stresses control flies had an increase in the frequency of muscle atrophy when compared to young non-stressed animals (approximately 2.5-4 fold increase). Young miRNA mutants had muscle atrophy already at 7 day, the frequency of which was approximately three times higher than in control. In addition, miRNA mutants showed loss of muscle integrity in response to sugar starvation and during aging, and the frequency of atrophic muscle appearance was six times higher than in young controls. This tendency of being extremely sensitive to aging was also observed in $m i R-927$ and $m i R-966$ mutants that also showed 6-fold increase in the frequency of atrophic muscle appearance in comparison to young controls (Supplementary Table 4). The frequency of muscle atrophy was observed in higher rate compared to the frequency of muscle degeneration. MiR-927 and miR-966 mutants did not show any differences in muscle degeneration phenotypes during temperature stress and sugar starvation, while an increase in 3-4 fold difference of atrophic muscles was observed compared to young control. Since miR-137 mutants showed an increase in muscle degeneration and muscle atrophy phenotypes already in young animals and these phenotypes were significantly progressing during aging, it indicates that this miRNA can be an especially good candidate to study further in order to elucidate the common mechanisms between MD and age-dependent muscle loss. 


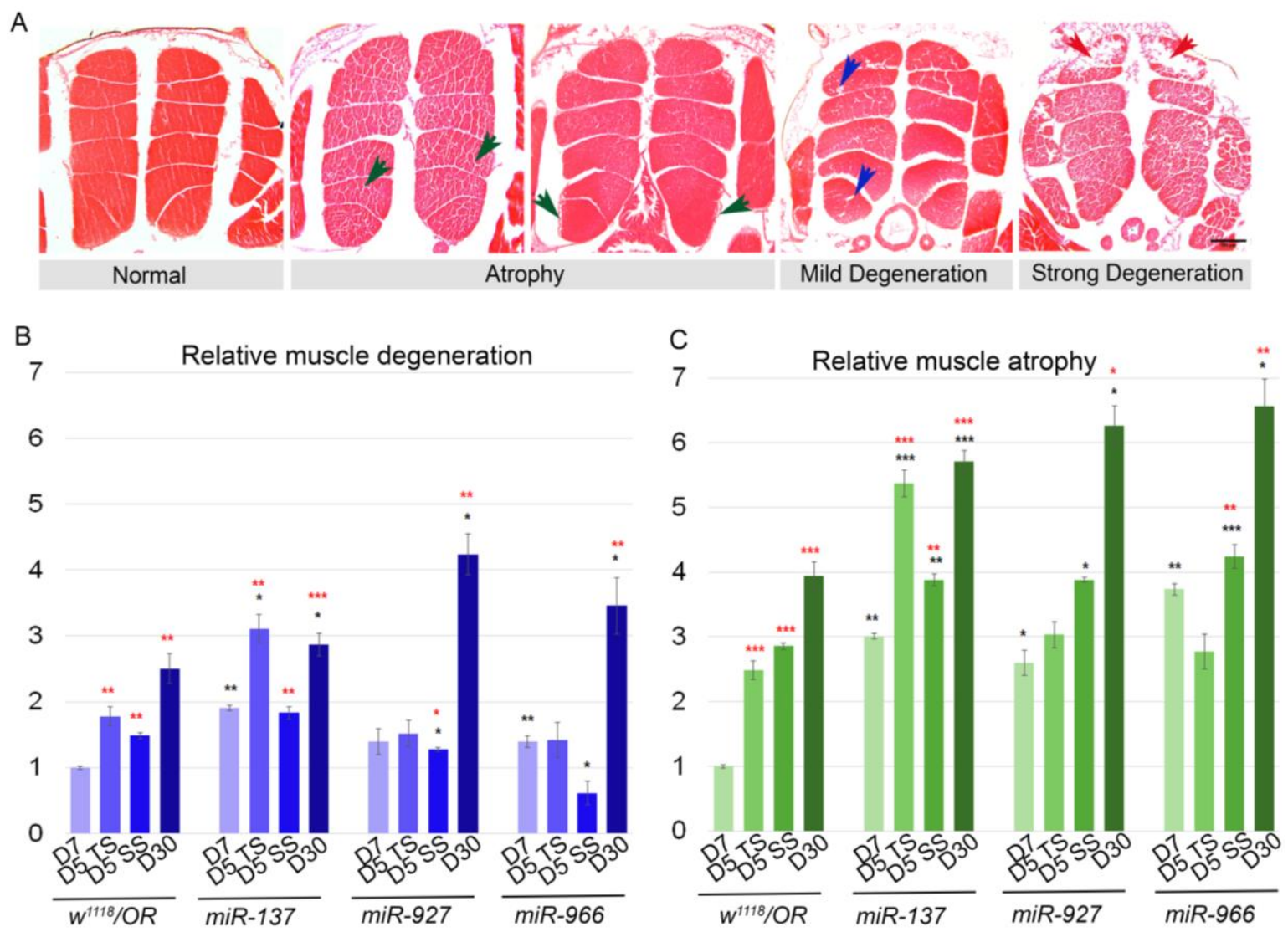

Figure 9. Loss of miRNA affects muscle maintenance

(A) Representative images of muscle degeneration and atrophic muscle phenotypes in adult Drosophila IFMs. Blue and the red arrows represent mild and strong muscle degeneration, while the green arrows represent muscle atrophy. (B) A bar graph representing relative muscle degeneration and $(\mathrm{C})$ relative muscle atrophy in different stresses compared to young control flies $\left(w^{1118} / O R\right)$. Stress enhances muscle degeneration and atrophy even in wild type flies. Control and $m i R-137$ mutants were responsive to all stresses when compared to young age (significance shown by red stars). In general, miR-137 mutants had $\sim 2$ times more muscle degeneration in young and sugar starvation and $\sim 3$ times more muscle degeneration in temperature stress and aging compared to young controls. Similarly, an increase in muscle atrophy by 3-5.5 fold was observed in miR-137 mutants when compared to young controls. Values are obtained from the averages of 3 biological replicates. Error bars represent $\mathrm{AVE} \pm \mathrm{SEM}$ and statistical significance was determined by two-tailed Student's t-test. $* \mathrm{P}<0.05, * * \mathrm{P}<0.01, * * * \mathrm{P}<0.001$ represent comparisons to control at the same condition, while $* \mathrm{P}<0.05, * * \mathrm{P}<0.01, * * * \mathrm{P}<0.001$ represent comparisons within the same genotype at a young age. $\mathrm{D} 7=$ young, 7 day old, $\mathrm{TS}=$ temperature stress, $\mathrm{SS}=$ sugar starvation, and $\mathrm{D} 30=$ aging, 30 day old. Scale bar $100 \mu \mathrm{m}$. See also Supplementary Table 4. 


\subsection{Conserved predicted targets of miRNAs are associated with multiple biological functions}

To verify whether $m i R-137$, $m i R-927$, and $m i R-966$ have muscle-related target genes to have function in muscle maintenance, we combined their putative conserved targets by TargetScan, release 6.2 (www.targetscan.org) to predict their possible biological roles related to muscle maintenance. Individual lists of predicted targets of all three miRNAs were processed separately in Generic Gene Ontology term finder tool (Boyle et al., 2004). MiR137 having the largest number of conserved targets (252 targets in total), which were found to be involved in the processes associated with multiple biological terms including synapse, neuromuscular junction, neuron, voltage-gated potassium complex, as well as transporter complex. Majority of miR-137 target genes were found to be associated with functions related to cell periphery and plasma membrane protein complex (Figure 10A). MiR-927 and miR-966 having 120 and 30 conserved targets, respectively, which were found to be involved in processes related to the cell periphery and cell membrane function (Figure 10B-C). Interestingly, among many genes that share similar associations, Dg is well known for its role in all of these processes that are coined for these miRNA predicted targets. 


\section{A}

component terms of miR-137 targets

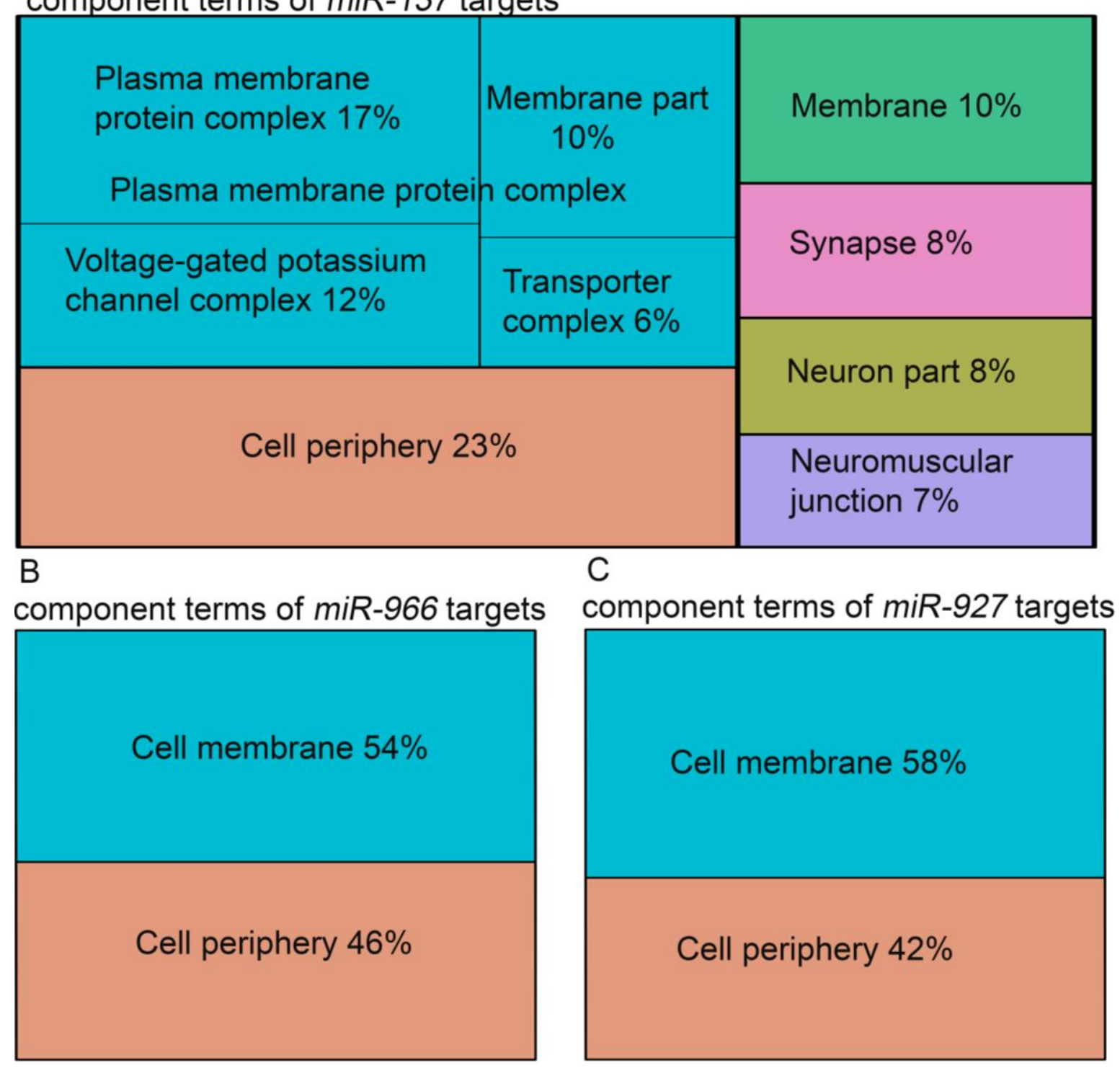

Figure 10. GO term for component processes for each miRNA targets

(A) Putative miR-137 targets are involved in plasma membrane protein complex, cell periphery or voltage-gated potassium channel complex and in neuronal functions related to the synapse and neuromuscular junction. (B-C) Putative targets of both miR-927 and miR966 are involved in the cell membrane- and cell periphery-related functions. 


\subsection{Ectopic expression of candidate miRNA affects muscle maintenance}

Our previous data showed that muscle integrity upon miRNA loss was severely compromised, indicating these miRNAs are required for normal muscle functions. The GO component terms further indicated that these miRNAs are required in majority of the functions related to the cell membrane and cell periphery related processes. In IFMs of Drosophila, Dg has been shown to be localized in muscle membrane (sarcolemma) and loss of Dg cause muscle degeneration and the phenotype is accelerated upon stress (Kucherenko et al., 2011). The study further identified genes that interact with the DGC components and shared functional similarity in the development of MDs. To further elucidate miRNAs affecting muscle maintenance by targeting muscle-specific target genes, we over-expressed our candidate miRNAs in muscle and scored for the muscle degeneration and atrophy phenotypes similar to its loss-of-function.

Over-expression of miRNAs in muscle was achieved by the UAS/Ga14 system. Two musclespecific-Gal4 lines were chosen to overexpress candidate miRNAs, one that drives the expression in adult muscle only and one that is active throughout the development: MhcGal4 and how-Gal4, respectively. Male flies of $U A S-m i R N A$ lines with virgin females of Mhc-Gal4 lines were crossed and the IFMs of progenies were analyzed. Mhc-Gal4 resides in X-chromosome; therefore, only females were analyzed for this experiment to reduce the dosage compensation effect on muscles. Using these two driver lines, we over-expressed $m i R-137, m i R-927$, and $m i R-966$ in muscles. Over-expression of $m i R-137$ and $m i R-927$ during development caused lethality at various developmental stages of flies (Supplementary Table 4). Ectopic expression of miR-137 with Mhc-Gal4 was semi-lethal and few escapers that were analyzed showed severe muscle degeneration and muscle loss, appearing as the complete absence of individual muscle (Figure 11). Over-expression of $m i R-927$ and $m i R$ 966 in adult muscle were not lethal for flies, however, the muscle integrity was severely compromised (Figure 12). On average, over-expression of miR-927 and miR-966 in adult muscle showed an increase in muscle degeneration (35-85\% and 22-60\%) compared to control flies (10-40\%) at young, temperature stress, sugar starvation, and aging (Figure 12A). Ectopic expression of $m i R-966$ using how-Gal4 resulted in 22-65\% cases of average muscle degeneration at young and various stresses compared to $10-40 \%$ cases observed in controls. In general, miR-966 over-expression with how-Gal4 ensued in much milder phenotype compared to Mhc-Gal4. 

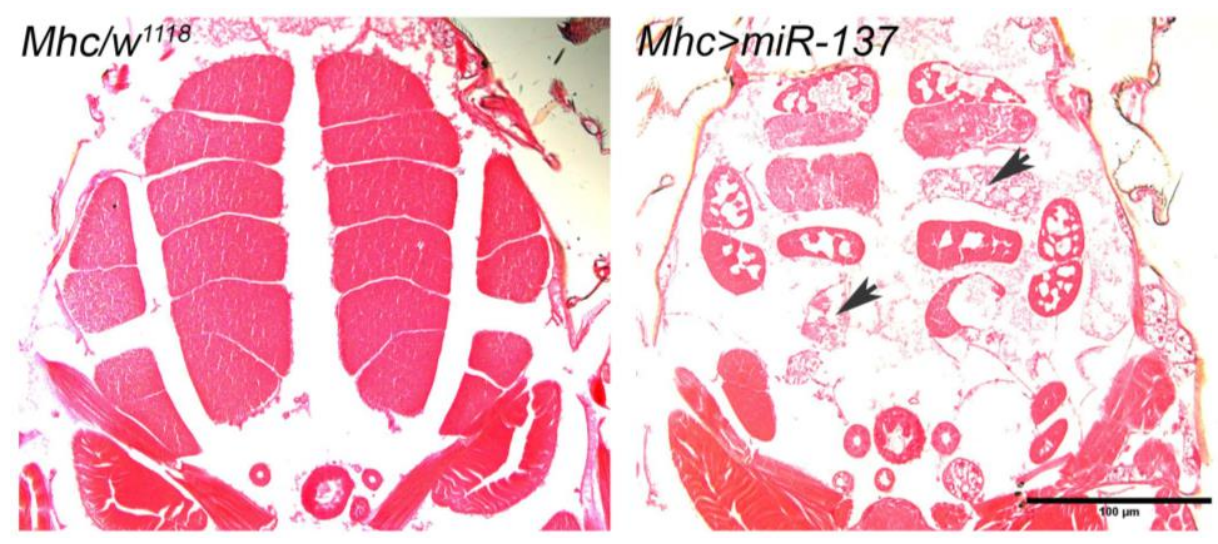

Figure 11. Over-expression of $m i R-137$ results in strong muscle degeneration and muscle loss

Ectopic expression of $m i R-137$ in adult fly muscle is semi-lethal to flies and few escapers that were analyzed show severe muscle degeneration or complete absence of an individual muscle (black arrow) as compared to control flies of genotype $M h c>w^{111}$, indicating level of $m i R-137$ in adult muscle must be maintained at the proper level. Scale bar $100 \mu \mathrm{m}$. See also Supplementary Table 4.

Over-expression of miRNAs also showed muscle atrophy phenotype. Over-expression of $m i R-927$ and miR-966 in adult muscle caused a significant increase in muscle atrophy (70$90 \%$ and $28-74 \%$ cases of atrophic muscles) compared to control (10-50\% cases of atrophic muscle) (Figure 12B). Over-expression of miR-966 during developmental stage resulted in relatively milder phenotype compared to over-expression during adult stage similar to the observation on muscle degeneration phenotypes (21-63\% atrophic muscle when overexpressed during development compared to $70-90 \%$ in adult over-expression). Together with the muscle degeneration and muscle atrophy phenotypes observed upon over-expression of $m i R-966$, it was concluded that miR-966 is required more for adult muscles maintenance. Similar to muscle degeneration, flies of all genotypes were sensitive to aging, indicating aging in general affects muscle maintenance. Altogether the data show that over-expression of identified miRNAs in our screen specifically in the muscle tissue resulted in severe phenotypes, indicating all of these candidate miRNAs can control at least one or more targets that are required for muscle maintenance and development. 

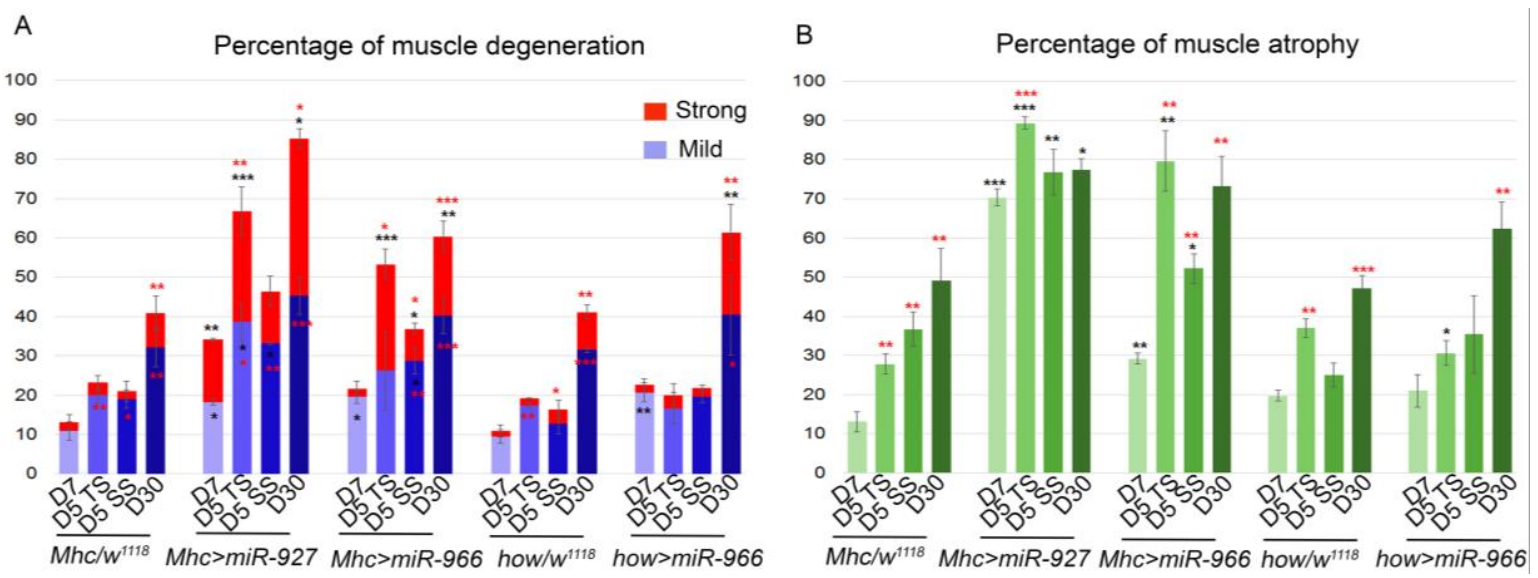

Figure 12. Over-expression of candidate miRNAs affects muscle maintenance

Bar graphs showing muscle degeneration (A) and muscle atrophy (B) phenotypes in adult IMFs. Over-expression of $m i R-927$ in adult muscle caused $35-85 \%$ of degenerated muscle and $70-90 \%$ of atrophic muscle, while over-expression of $m i R-966$ caused $20-60 \%$ of degenerated muscle and 20-80\% atrophic muscle. Over-expression of miR-966 was more detrimental to flies at adult stage compared to over-expression during developmental stage. Values are obtained from the averages of 3 biological replicates. Error bars represent $\mathrm{AVE} \pm \mathrm{SEM}$ and statistical significance was determined by two-tailed Student's t-test. $* \mathrm{P}<0.05, * * \mathrm{P}<0.01, * * * \mathrm{P}<0.001$ represent comparisons to control at the same condition, while $* \mathrm{P}<0.05, * * \mathrm{P}<0.01, * * * \mathrm{P}<0.001$ represent comparisons within the same genotype at a young age. $\mathrm{D} 7=$ young, 7 day old, $\mathrm{TS}=$ temperature stress, $\mathrm{SS}=$ sugar starvation, and $\mathrm{D} 30=$ aging, 30 day old. See also Supplementary Table 4.

\subsection{Candidate miRNAs target Dg-3' UTR in vitro}

In order to validate that miRNAs identified in our screen ( $m i R-137, m i R-927$, and $m i R-966)$ can indeed regulate $D g$ by targeting its 3'UTR, Drosophila S2 cell-based luciferase reporter assay was carried out. According to FlyBase, a database for Drosophila gene and genome (http://flybase.org/), Dg transcript has two different 3'UTRs: the long 3'UTR is $2102 \mathrm{bp}$ long (Figure 10A) and the short 3'UTR is 1609 bp long. About $60 \%$ of $D g$ transcripts had been shown to contain long 3'UTR (Yatsenko et al., 2014). To study all miRNA based regulation possibilities, the long 3'UTR was divided into four smaller fragments (S1-S4). This made cell culture analyses easier and made it accessible to study individually selected miRNAs based on the predicted position of the miRNA binding sites.

$M i R-927$ is predicted to have a binding site on long $D g-3$ ' $U T R$, while both $m i R-137$ and $m i R$ 966 have predicted binding sites on short $D g-3$ 'UTR. Importantly, miR-137 and miR-927 are predicted to have multiple binding sites in $D g-3$ ' $U T R$ (highlighted with marron box and blue box, Figure 13A), while miR-966 is predicted to have only one binding site (highlighted with yellow box). To test if all three miRNAs can target $D g$ in vitro, plasmid containing sequence 
that include individual miRNA predicted binding sites were transfected in S2R+cell lines. S3 fragment of Dg-3'UTR did not contain any predicted binding sites for these three miRNAs; therefore, S3 fragment was used as a negative control and was transfected with the empty psiCHECK $K^{T M}-2$ vector. In addition, $m i R-956$ was chosen as a negative control as it is not predicted to have any binding sites on Dg 3'UTR. Both renilla and firefly luminescence light signals encoded by the constructed plasmid transcripts with and without miRNA binding sites were measured. The relative signal intensity values showed the significant downregulation of corresponding $D g$ transcripts by all three miRNAs ( $m i R-137, m i R-927$, and miR-966), indicating that $D g$ can be targeted by all these miRNAs in vitro (Figure 10B). As a negetive control, miR-956 that is not predicted to have any binding sites on Dg-3 'UTR was used. It failed to downregulate the luciferase signal, supporting in silico predictions for miRNA binding sites.
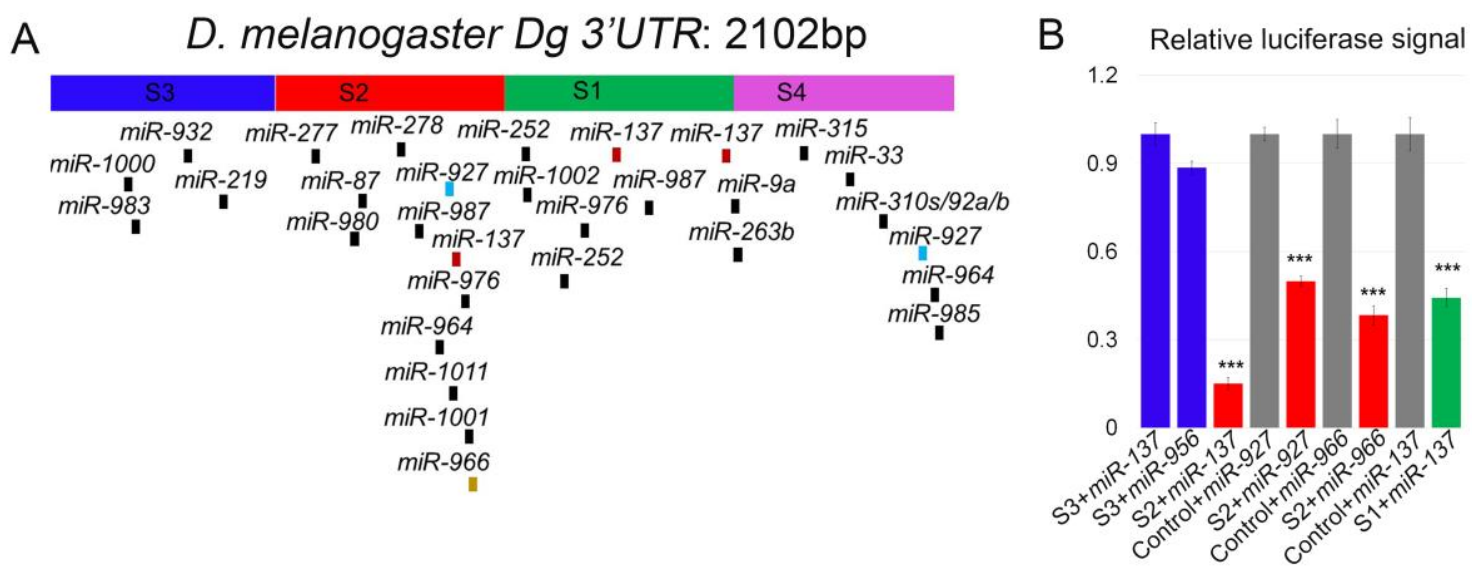

\section{Figure 13. Selected miRNAs can target $D g$ in vitro}

(A) Predicted miRNA binding sites in $D g$ 3'UTR, which is subdivided into four regions corresponding to constructs used in the luciferase assay (S1-S4). Out of all the miRNAs that are predicted to target $D g, m i R-137$ is predicted to have 3 binding sites (highlighted in marron), miR-927 has 2 binding sites (highlighted in light blue), and miR-966 has 1 binding site (highlighted in yellow) in Dg 3'UTR. (B) To calculate relative downregulation by miRNAs, a reporter luciferase values were calculated from raw readouts of Renilla to Firefly luciferase values. The background control values (transfection with empty psiCHECK ${ }^{T M}-2$ with no reporter construct) were subtracted from respective readouts. Obtained values were then normalized to empty $p s i C H E C K^{T M}-2$ to the values of $p s i C H E C K^{T M}-2-D g-3$ 'UTR (S1S4) in the presence of miRNAs. Error bars represent AVE \pm SEM, and statistical significance was determined by two-tailed Student's t-test $\left(* \mathrm{P}<0.05,{ }^{*} * \mathrm{P}<0.01, * * * \mathrm{P}<0.001\right)$. See also Supplementary Table 5. 


\subsection{Downregulation of Dg affects muscle maintenance}

The precise level of Dg has been implicated in many neuronal and muscular dysfunctions (Yatsenko et al., 2014; Yatsenko and Shcherbata, 2014). Our in vitro data demonstrated that all three miRNAs (miR-137, miR-927, and miR-966) can target Dg 3 'UTR and the analysis of GO component terms of conserved targets further suggests their involvement in processes related to the cell membrane and cell periphery. We have also shown that over-expression of miRNAs resulted more severe phenotype compared to their loss, indicating these miRNA can regulate muscle-specific genes. Altogether, we hypothesized that the precision of $\mathrm{Dg}$ expression in adult muscle especially in repsonse to stress is maintained by these miRNAs. The previous study has shown that complete loss of $D g$ causes muscle degeneration and this phenomenon is enhanced upon stress conditions (Kucherenko et al., 2011). However, phenotypes caused by downregulation of a gene often results in milder phenotypes than its complete loss. Therefore, to further verify the role of $\mathrm{Dg}$ in muscle maintenance, we downregulated Dg specifically in adult muscle and scored for muscle degeneration and muscle atrophy phenotypes in young and aged flies as described in chapter 3.1.3.

Downregulation of $\mathrm{Dg}$ in adult muscle caused muscle degeneration and muscle atrophy already in young flies and the phenotype was enhanced during aging (Figure 14A). IMFs analyzed in young and aged flies of $M h c>D g^{R N A i}$ genotype had approximately 1.5-2 times increase in muscle degeneration when compared to the control genotype (Mhc/OR). Similar increase in muscle atrophy was observed in young and aged flies upon downregulation of Dg. As observed before, both genotypes were sensitive to aging and showed an increase in muscle degeneration and atrophy phenotypes (Figure 12B). Overall, this result further concluded that downregulation of $\mathrm{Dg}$ is causing muscle degeneration similar to its loss of function and also affects muscle maintenance. Both phenotypes progress in an agedependent manner. 
A

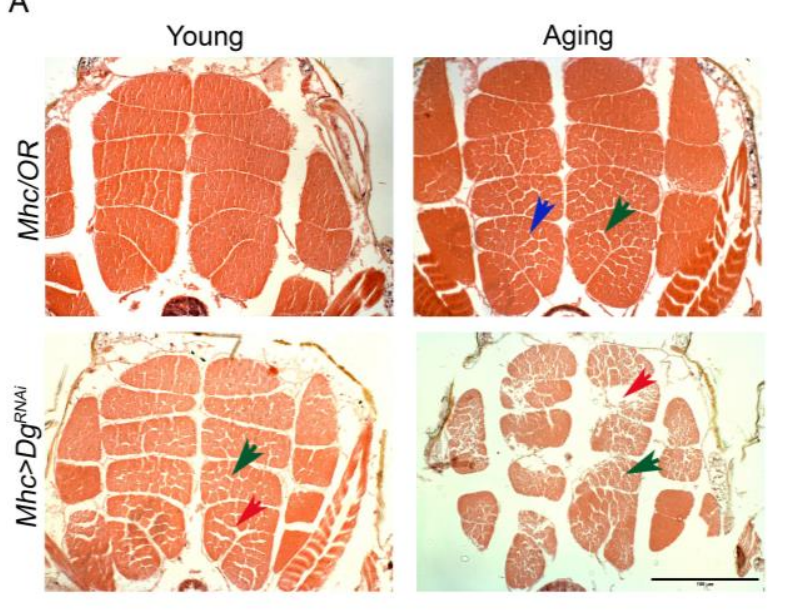

B

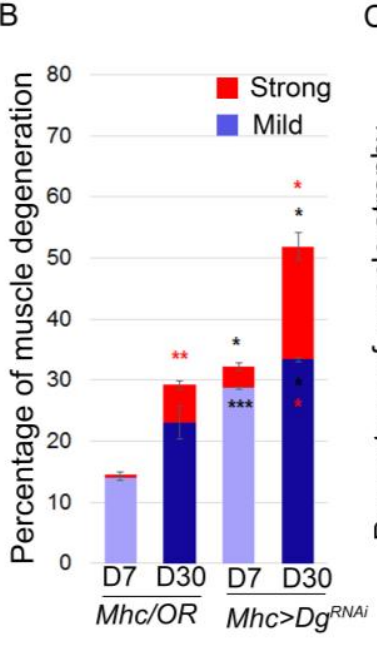

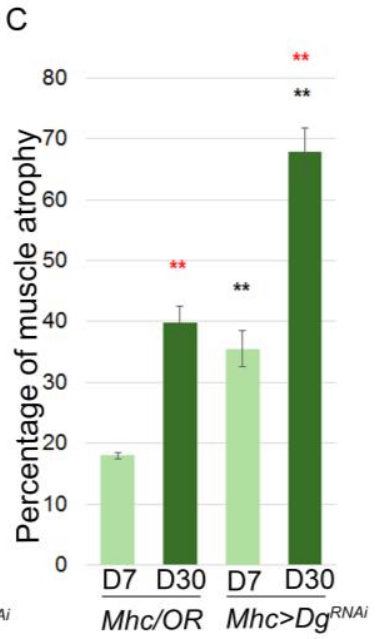

Figure 14. Downregulation of Dg shows age-dependent loss of muscle integrity

(A) IMFs of adult Drosophila showing mild and strong muscle degeneration phenotypes (indicated by blue and red arrows) and muscle atrophy phenotype (indicated by green arrows) in control $(M h c / O R)$ and $M h c>D g^{R N A i}$ flies. Bar graphs showing the frequency of muscle degeneration (B) and muscle atrophy (C). On average, 1.5-2 times increase in muscle degeneration and muscle atrophy was observed upon downregulation of $\mathrm{Dg}$ in young and aged animals compared to control. Both genotypes show age-dependent muscle maintenance phenotypes with an average of two fold increase as compared to young animals. Values are obtained from the averages of 3 biological replicates. Error bars represent AVE $\pm \mathrm{SEM}$ and statistical significance was determined by two-tailed Student's t-test. ${ }^{*} \mathrm{P}<0.05,{ }^{*} * \mathrm{P}<0.01$, $* * * \mathrm{P}<0.001$ represents comparisons to control at the same condition, while $* \mathrm{P}<0.05$, $* * \mathrm{P}<0.01, * * * \mathrm{P}<0.001$ represent comparisons within the same genotype at a young age. $\mathrm{D} 7=$ young, 7 day old, TS= temperature stress, $\mathrm{SS}=$ sugar starvation, and $\mathrm{D} 30=$ aging, 30 day old. Scale bar $100 \mu \mathrm{m}$. See also Supplementary Table 4.

\subsection{Dissecting biological roles of $\mathrm{miR}-137$}

$M i R-137$ was further followed up to decipher its role in MDs with various reasons: 1) $\mathrm{miR}$ 137 mutants had an increase in $D g$ and $D y s$ mRNA levels, 2) the severity of muscle maintenance phenotypes were ameliorated in miR-137 mutants as well as upon its overexpression compared to $m i R-927$ and $m i R-966$, indicating $m i R-137$ is a better candidate to have role in regulating muscle-specific genes, 3) $m i R N A-137$ mutants were responsive to various stresses, indicating it is required to address negative effects of stress, 4) it can target Dg in vitro, and 5) $m i R-137$ is predicted to have multiple functions including neuronal, cell membrane and cell periphery related biological processes, for which the precision of $\mathrm{Dg}$ expression has been shown to essential. 


\subsubsection{MiR-137 is conserved among higher eukaryotes}

MiR-137 is well conserved among animal kingdom (Figure 15). Drosophila miR-137 is predicted to have 252 conserved targets (TargetScanFly, Release 6.2), among which dystroglycan and sarcoglycan are common predicted targets in higher eukaryotes, including humans, mouse, zebrafish, and worms, which implies that miR-137 can share similar functions in these organisms. Besides $D g$, mei-P26, N-methyl-D-aspartate (NMDA) receptor (NMdar2), dopamine receptor $(D 2 R)$, and $\gamma$-aminobutyric acid (GABA) receptor (GABA-BR3) are among other verified targets (in vitro) of miR-137 in flies (Herranz et al., 2010; Kong et al., 2015).

\section{Mature miRNAs and their seed sequences}

\section{dme-miR-137-3p UAUUGCUUGAGAAUACACGUAG \\ hsa-miR-137 UAUUGCUUAAGAAUACGCGUAG \\ mmu-miR-137-3p UAUUGCUUAAGAAUACGCGUAG \\ dre-miR-137-3p UUAUUGCUUAAGAAUACGCGUA \\ cel-miR-234-3p UAUUGCUCGAGAAUACCCUUXX}

Figure 15. MiR-137 has conserved seed as well as mature miRNA sequences

The mature Drosophila miRNA together with its human, mouse, zebrafish, and worm orthologues shows the conservation of the seed sequence and the mature miRNA sequence among different animal species.

\subsubsection{MiR-137 is expressed in larval muscle, brain, and testis}

MiRNAs are known for expression tuning and expression buffering of their target mRNAs. In Drosophila, it has been shown previously that miR-310s buffers the expression of $\mathrm{Dg}$ in in larval brain and influence the proper formation of nervous tissue (Yatsenko et al., 2014). Similarly, it has been reported that precise level of $\mathrm{Dg}$ to form myotendious junction (MTJs) in fly embryo is regulated by $m i R-9 a$ (Yatsenko and Shcherbata, 2014). Studies have shown that $\mathrm{Dg}$ is virtually expressed in all tissues, indicating its function is much more diverse and complex in the whole organism. Therefore, we further investigated the biological roles of $m i R-137$ and characterized its function in relation to diverse phenotypes observed in MD and dystroglycanopathies. 
First, we analyzed its expression patterns in different tissues. In situ hybridization as well as fluorescence in situ hybridization (FISH) was performed using miR-137-3p LNA probe. Abdominal segments A3-A5 of muscle 5, 6, and 7 were analyzed to check the miR-137 expression in larval muscle. FISH showed that $m i R-137$ is expressed in larval muscle and its expression was concentrated more at the edge of the muscle wall, possibly at the NMJ (Figure $\left.16 \mathrm{~A}-\mathrm{A}^{\prime}\right)$. In the $m i R-137^{k o}$ mutants, used as a negative control, no expression pattern in muscle was observed, verifying the specificity of LNA probe (Figure 16 B-B').

In situ hybridization showed the expression of $m i R-137$ in nervous system. It is expressed in several cell types presumably in type I/II neuroblasts, Kenyon cells of the mushroom body, abdominal neuromeres, and dorsal cell bodies of the larval brain (Figure 16C). The expression pattern in the larval brain suggests that $m i R-137$ also could have a role in neuronal processes. MiR-137 expression was also detected in the apical portion of the adult fly testis (Figure 16E-E'). The apex of adult fly testes is composed of a pool of germline as well as somatic cells surrounding the hub. Cells next to the hub are stem cells in nature, while the cells away from the hub are differentiating gonialblast encapsulated with two somatic cells. Since miR-137 LNA probe was concentrated only on a subset of the cells in the apex and appeared as dots (Figure 16E'), it was assumed that miR-137 is possibly expressed in early somatic cells of fly testes. The expression patterns of $m i R-137$ further suggest the involvement of $m i R-137$ in the neuronal, cell membrane and cell periphery related processes as coined by GO component terms of its predicted mRNA targets.

The role of Dg has been well studied in larval NMJs and brain. It will be interesting to study further the relationship between $m i R-137$ and $\mathrm{Dg}$ and their shared function not only in the muscle, but also in the nervous system and in spermatogenesis. 

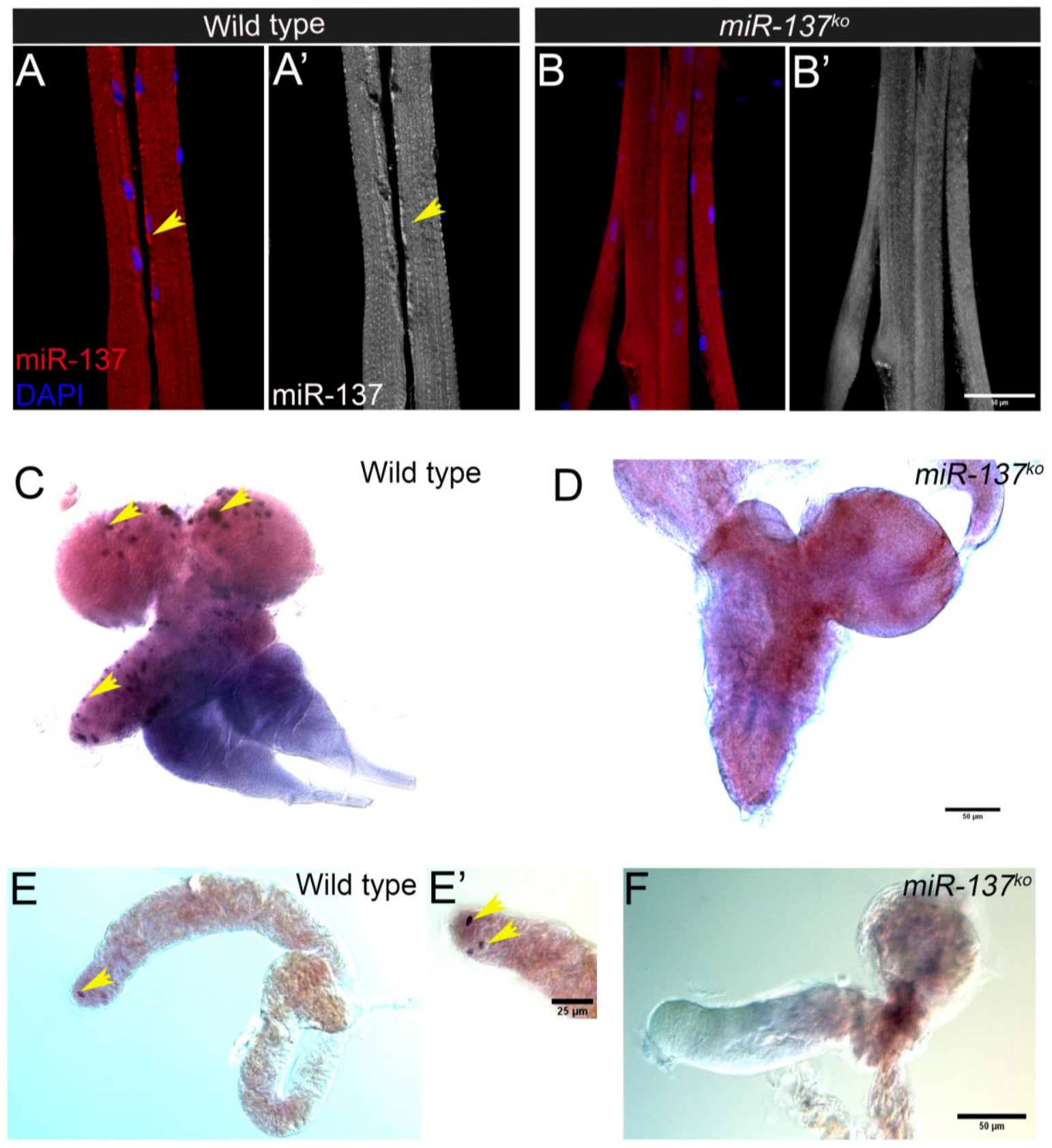

\section{Figure 16. MiR-137 expression patterns}

In situ hybridization (ISH) and Fluorescence in situ hybridization (FISH) in wild type flies using LNA probe for mature $m i R-137$ shows that $m i R-137$ is expressed in larval muscle and brain as well as in adult testis (indicated by yellow arrows) (A-C-E). FISH shows that $m i R$ 137 is expressed in larval muscle; in particular in neuromuscular junctions (NMJs). Calorimetric reaction of $m i R-137$ LNA probe shows expression of $m i R-137$ in L3 larval brain (C) presumably in type I/II neuroblasts, Kenyon cells of the mushroom body, abdominal neuromeres, and dorsal cell bodies as well as in adult fly testes (E-E'), particularly, in early somatic cells at the apex of the testis. Contrary, the absence of fluorescence as well as calorimetric signal was noted in $m i R-137$ mutants (B-D-F). Scale bar $50 \mu \mathrm{m}$. 


\subsubsection{MiR-137 affects muscle maintenance}

\subsubsection{Downregulation of $m i R-137$ is more detrimental for adult muscle maintenance}

To confirm the specificity of role of $m i R-137$ in adult muscles to maintain its integrity during adulthood and in response to stress, we down-regulated miR-137 in fly muscle. Muscle degeneration, as well as muscle atrophy phenotype, was scored as described in Figure 9A. Down-regulation of $m i R-137$ was achieved using $U A S-m i R-137^{\text {sponge }}$. Sponge contains multiple miRNA binding sites with central bulges to stably sequester the miRNA in vivo. For most miRNAs, sponge had been shown to produce accurate but milder version of lossof-function phenotype (Cohen, 2009).

Downregulation of $m i R-137$ resulted in phenotypes similar to its loss-of-function. This further favored that $m i R-137$ is important for muscle maintenance. Mhc-Gal4 $>$ miR-137sponge flies showed 1.5-3 times increase in incidence of muscle degeneration and 3-5 times increases in incidence of muscle atrophy under young and stress conditions. Similar comparison was made on how $>m i R-137^{\text {sponge }}$ flies. The calculated phenotype showed about 1.5 times increase in incidence of muscle degeneration and about 2 times increase in muscle atrophy compared to controls. It was also noted that down-regulation of $m i R-137$ during developmental stage resulted in relatively milder phenotype compared to its downregulation in adult muscle, indicating miR-137 contributes to muscle maintenance during adult stage. Over-expression of Dg in adult muscle was relatively milder compared to downregulation of miR-137. The calculated phenotype showed about 1.5-2 times increase in muscle degeneration and muscle atrophy compared to controls (Figure 17). However, overexpression of Dg during development using how-Gal4 driver was semi-lethal and few escapers that were analyzed showed fused muscle phenotype (Supplementary Figure 1) rather than the muscle degeneration or atrophy phenotypes, indicating over-expression of Dg during developmental stage does not cause MD development. Overall, it was concluded that both over-expression and downregulation of $m i R-137$ is detrimental for muscle maintenance, indicating $m i R-137$ is required for muscle maintenance. Similarly, both loss-of-function and downregulation, as well as over-expression of $\mathrm{Dg}$, are detrimental to flies, indicating, the precise level of Dg needs to be maintained for healthy musculature.

The experiment further showed that phenotypes caused in adult muscle due to downregulation of $m i R-137$ can be partially rescued by Dg over-expression. 

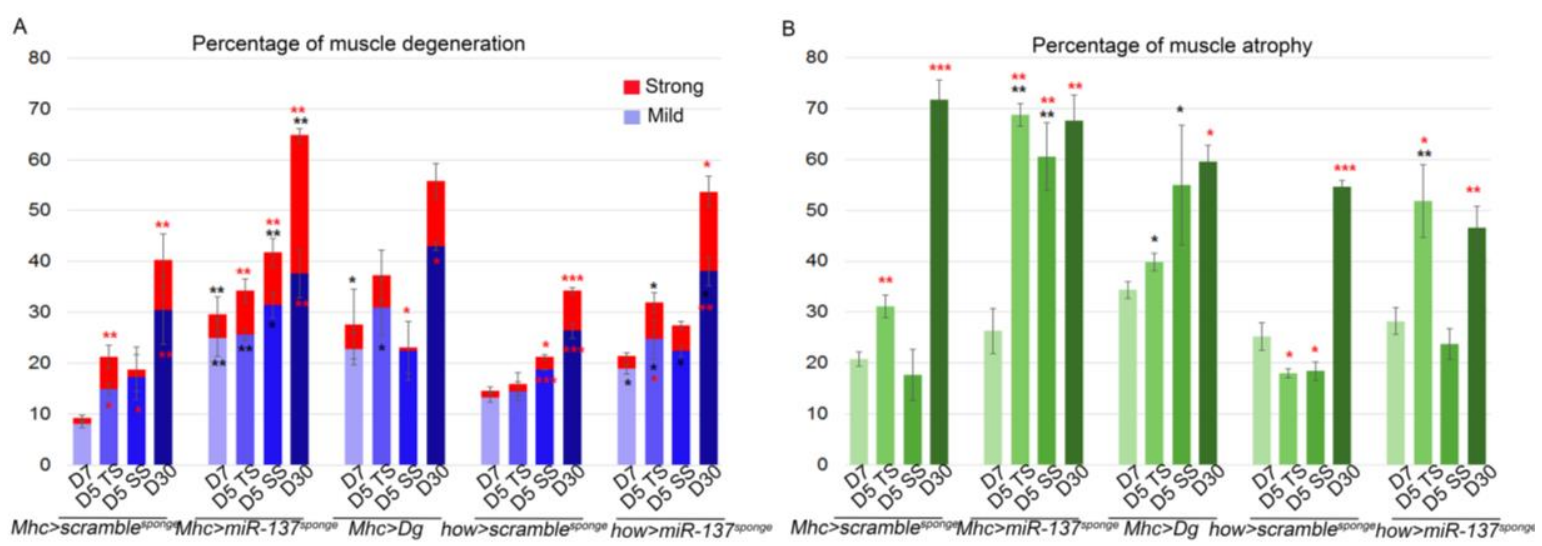

Figure 17. MiRNA-137 regulates Dg levels for muscle maintenance

Bar graphs showing frequency of muscle degeneration (A) and muscle atrophy (B) in IFMs of Drosophila. Downregulation of miR-137 specifically in muscle leads to muscle degeneration as well as muscle atrophy phenotype. Both phenotypes were more severe as compared to over-expression of Dg in muscle. Downregulation of miR-137 using Mhc-Gal4 also resulted in an increase in muscle and atrophy phenotypes as compared to the control indicating, $m i R-137$ is required for regulation of genes expressed in muscles. Values are obtained from the averages of 3 biological replicates. Error bars represent AVE \pm SEM and statistical significance was determined by two-tailed Student's t-test. ${ }^{*} \mathrm{P}<0.05,{ }^{*} * \mathrm{P}<0.01$, $* * * \mathrm{P}<0.001$ represents comparison to control at the same condition, while ${ }^{*} \mathrm{P}<0.05$, $* * \mathrm{P}<0.01, * * * \mathrm{P}<0.001$ represents comparison within the same genotype at a young age. $\mathrm{D} 7=$ young, 7 day old, TS= temperature stress, $\mathrm{SS}=$ sugar starvation, and D30= aging, 30 day old. See also Supplementary Table 4.

\subsubsection{Downregulation of $\mathrm{Dg}$ in $\mathrm{miR}-137$ loss-of-function mutants rescues the muscle maintenance phenotypes}

To further verify that $\mathrm{Dg}$ level must be regulated in adult muscle to maintain healthy musculature, we analyzed $m i R-137$ heterozygous mutants ( $m i R-137^{\mathrm{ko} / \mathrm{Df}}$ ) that have reduced level of $\mathrm{Dg}$ by one copy. MiR-137 resides on the second chromosome, $16 \mathrm{kbs}$ away from $D g$ genomic locus (http://flybase.org/). The deficiency line available at BDSC (Df(2R)ED2457) referred as $m i R-137^{D f}$ apart from removing $m i R-137$, also has genomic locus of $D g$ deleted (Figure 18). The $m i R-137^{k o}$ line, however, only affects $m i R-137$ locus and replaces with miniwhite gene (Chen et al., 2014). Hence, $m i R-137^{k o / D f}$ lines have one $D g$ copy loss compared to homozygous $m i R-137^{k o}$ lines that have both $D g$ copies intact. Therefore, I analyzed and compared muscle maintenance phenotypes in $m i R-137^{k o}$ and $m i R-137^{k o / D f}$ to further pinpoint that $\mathrm{Dg}$ is regulated by $m i R-137$.

As expected, $m i R-137^{k o / D f}$ flies had a much milder phenotype compared to $m i R-137^{k o}$ lines.

The calculated phenotype include average muscle degeneration of $12-35 \%$ in control at young and stress conditions, while $m i R-137^{k o}$ flies had up to $35-55 \%$ of muscle degeneration. 
This phenotype was relatively reduced in $m i R-137^{k o / D f}$ lines with an average frequency of muscle degeneration ranging $30-45 \%$. The effect of muscle maintenance upon reduced level of $D g$ was not visible in young flies, however upon stress the phenotypes were relatively milder in $m i R-137^{k o / D f}$ compared to $m i R-137^{k o}$ lines (35-55\% in $m i R-137^{k o}$ compared to 30$45 \%$ (Figure 19A). The severity of the phenotypes in $m i R-137^{k o / D f}$ were statistically lower than $m i R-137^{k o}$ flies under stress conditions, indicating a partial rescue of muscle maintenance phenotype due to one copy loss of $D g$. Interestingly, downregulation of Dg by one copy in miR-137 loss-of-function background could not rescue muscle atrophy phenotype suggesting that other than $D g$, more miR-137 dependent targets could be involved in regulation of muscle size upon stress (Figure 19B).

qRT-PCR on thoraces of young flies further revealed 2-3 times increase in $D g$ mRNA levels in $m i R-137^{k o / D f}$ and $m i R-137^{k o}$ lines compared to control. The level of Dg in $m i R-137^{k o / D f}$ was relatively lower as compared to $m i R-137^{k o}$ (Figure 19C). Overall, this experiment indicates that that $D g$ is a bona fide target $m i R-137$ in muscles.

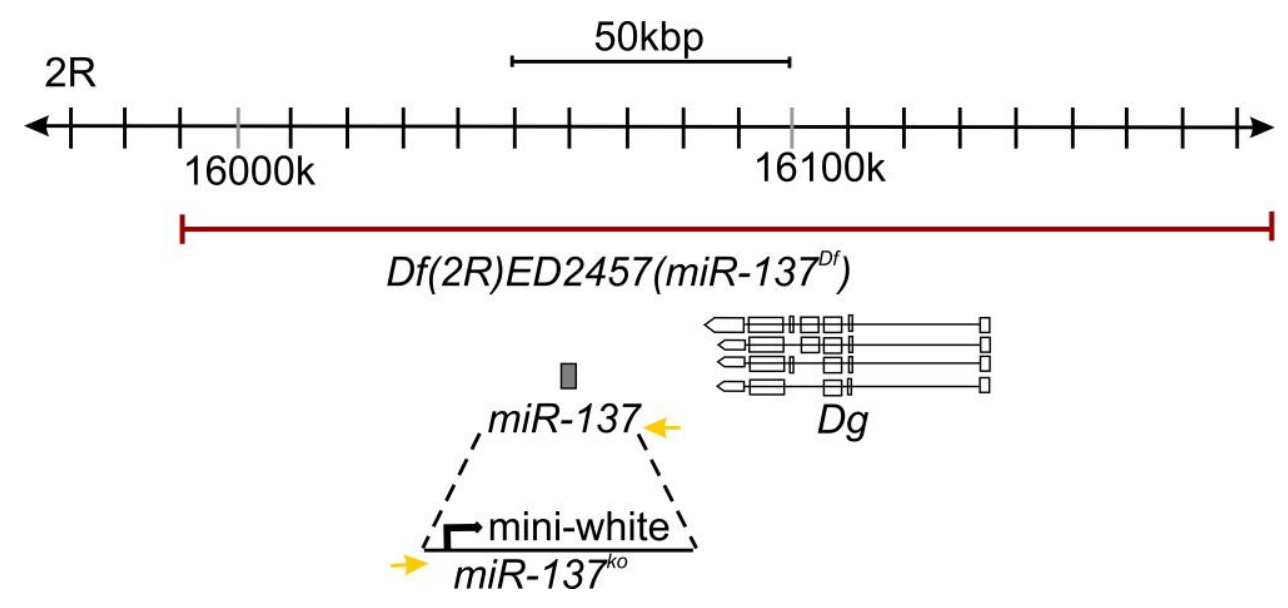

\section{Figure 18. Genomic locus of $m i R-137$}

MiR-137 is located on the minus strand of chromosome $2 \mathrm{R}$, and the $m i R-137^{k o}$ line was made using $p R M C E$ (recombinase-mediated casette exchange), which replaces miR-137 with miniwhite gene. $M i R-137^{D f}$ genomic locus $D f(2 R) E D 2457$, available from BDSC, uncovers a large genomic region including $m i R-137$ and $D g$. For use in all phenotypic analyses, the $m i R$ $137^{k o}$ line was backcrossed in the $w^{1118}$ background for 8 generations, and the final stocks were verified using primers that detect mini-white and the miR-137 locus (yellow arrows). 

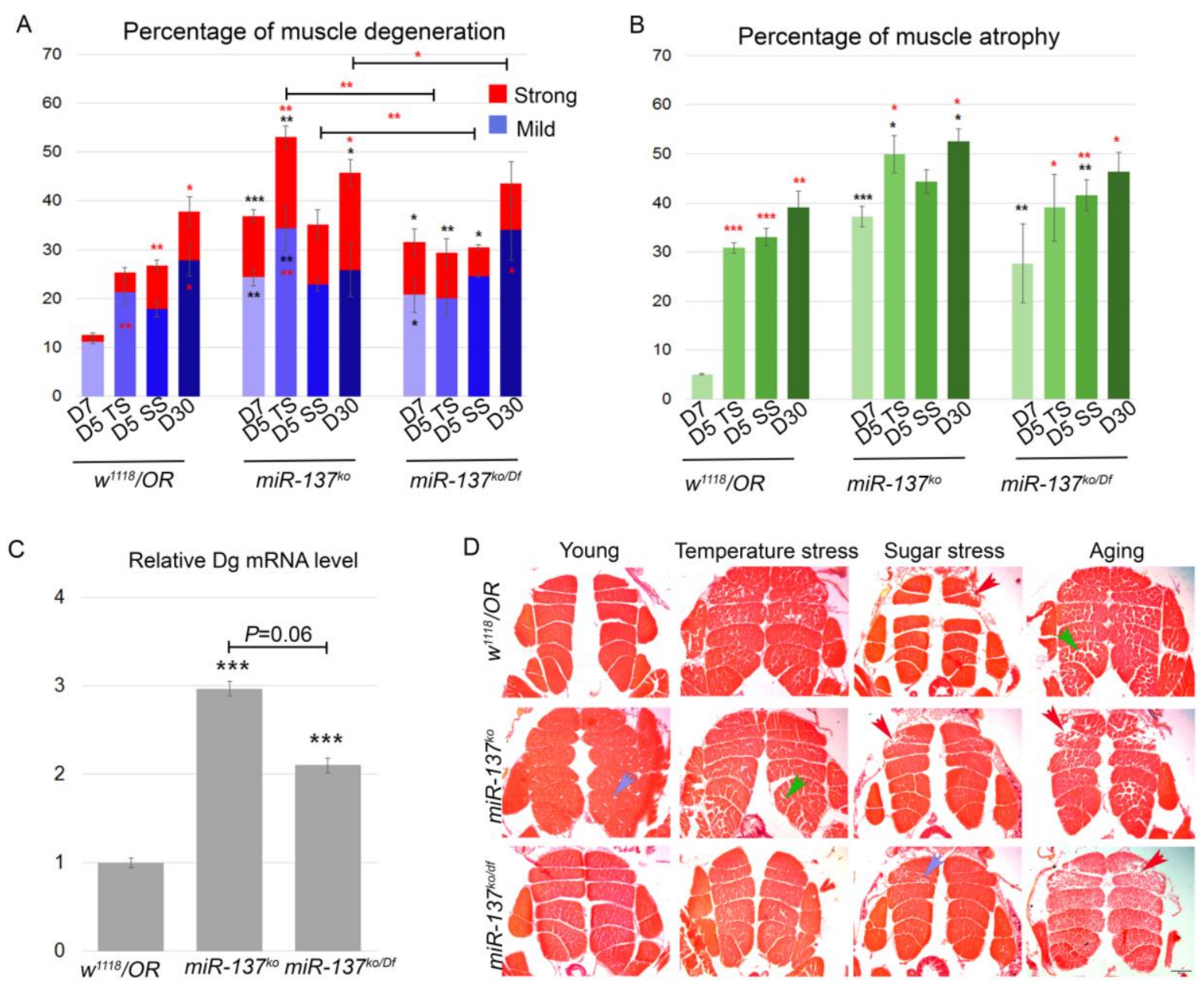

Figure 19. $D g$ is a bona fide target of $m i R-137$ in muscle

A bar graph showing muscle degeneration (A) muscle degeneration and (B) muscle atrophy phenotypes in miR-137 mutants and control. MiR-137 $7^{k o / D f}$ lines can rescue muscle degeneration as well as muscle atrophy phenotypes of $m i R-137^{k o}$. (C) qRT-PCR shows that the mRNA level of $D g$ is significantly increased in $m i R-137^{k o}$ and $m i R-137^{k o / D f}$ lines. No significant difference is detected between the homozygous and trans-heterozygous knockout lines. (D) Representative images of IMFs of control, $m i R-137^{k o}$, and $m i R-137^{\mathrm{ko} / D f}$ lines showing mild and strong muscle degenerations (blue and red arrows, respectively) as well as, muscle atrophy (green arrows) phenotypes in young, temperature stress, sugar starvation, and aging conditions. Values are obtained from the averages of three biological replicates. Error bars represent $\mathrm{AVE} \pm \mathrm{SEM}$ and statistical significance was determined by two-tailed Student's t-test. $* \mathrm{P}<0.05, * * \mathrm{P}<0.01, * * * \mathrm{P}<0.001$ represents comparison to control at the same condition, while $* \mathrm{P}<0.05, * * \mathrm{P}<0.01, * * * \mathrm{P}<0.001$ represents comparison within the same genotype at a young age. $\mathrm{D} 7=$ young, 7 day old, $\mathrm{TS}=$ temperature stress, $\mathrm{SS}=$ sugar starvation, and D30= aging, 30 day old. See also Supplementary Table 4. 


\subsubsection{MiR-137 mutants have perturbed spermatogenesis}

\subsubsection{MiR-137 affects early somatic cell numbers in adult fly testis}

The somatic cells of testes provide essential support to the germline and have been implicated in proper spermatogenesis. Due to the expression pattern of $m i R-137$, presumptively detected in early somatic cells of the adult testis (Figure 16), we investigated whether the loss of miR-137 causes defective spermatogenesis. Adult testes of 8-12 day old flies were dissected and stained with an early somatic cell marker Traffic jam (Tj) as well as a late somatic cell marker Eyes absent (Eya). We found that control animals maintain constant early somatic cell population $(56.3 \pm 2.0)$ and a late somatic cell population $(84.0 \pm$ 1.2) per testis, whereas $m i R-137$ mutants have significantly more early somatic cells $(82.2 \pm$ $1.5)$ and nearly the same number of late somatic cells $(80.4 \pm 0.5)$ per testis (Figure 20C). Both $\mathrm{Tj}$ and Eya can be visualized in 4-8 cell stage spermatocysts. However, quantification of cells co-stained with both anti-Tj and anti-Eya antibodies revealed statistically insignificant population of early somatic cells $(6.4 \pm 0.5$ compared to $11.1 \pm 2.0)$ between the control and miR-137 mutants, respectively. The visible differences were seen in a total somatic cell population in $m i R-137$ mutants $(151.6 \pm 0.1)$ compared to control $(134.2 \pm 2.9)$. The total increase in somatic cells was therefore solely due to an increase in early somatic cell numbers, while late somatic cell numbers do not change (Figure 20B-B'). Therefore, for further analysis, only early somatic cells, found at the apical region approximately $145 \mu \mathrm{m}$ away from the hub, were counted. The increase in the early somatic cell population can be due to either an increase in proliferation or a delay in differentiation. Among somatic cells, only CySCs proliferate in wild type testes. Therefore, we stained control and mutant testes with the mitotic marker Phospho-Histone H3 (PH3). Proliferating somatic cells were found only near the hub therefore, no obvious difference in increase in proliferation in $m i R-137$ mutant testes was concluded (data not shown). We further stained the control and mutant testes with spectrosome and fusome marker, Adducin (Add) to mark mitotically active cells and found increase in differentiating germline, further supporting our latter hypothesis of delayed differentiation (Supplementary Figure 2). 

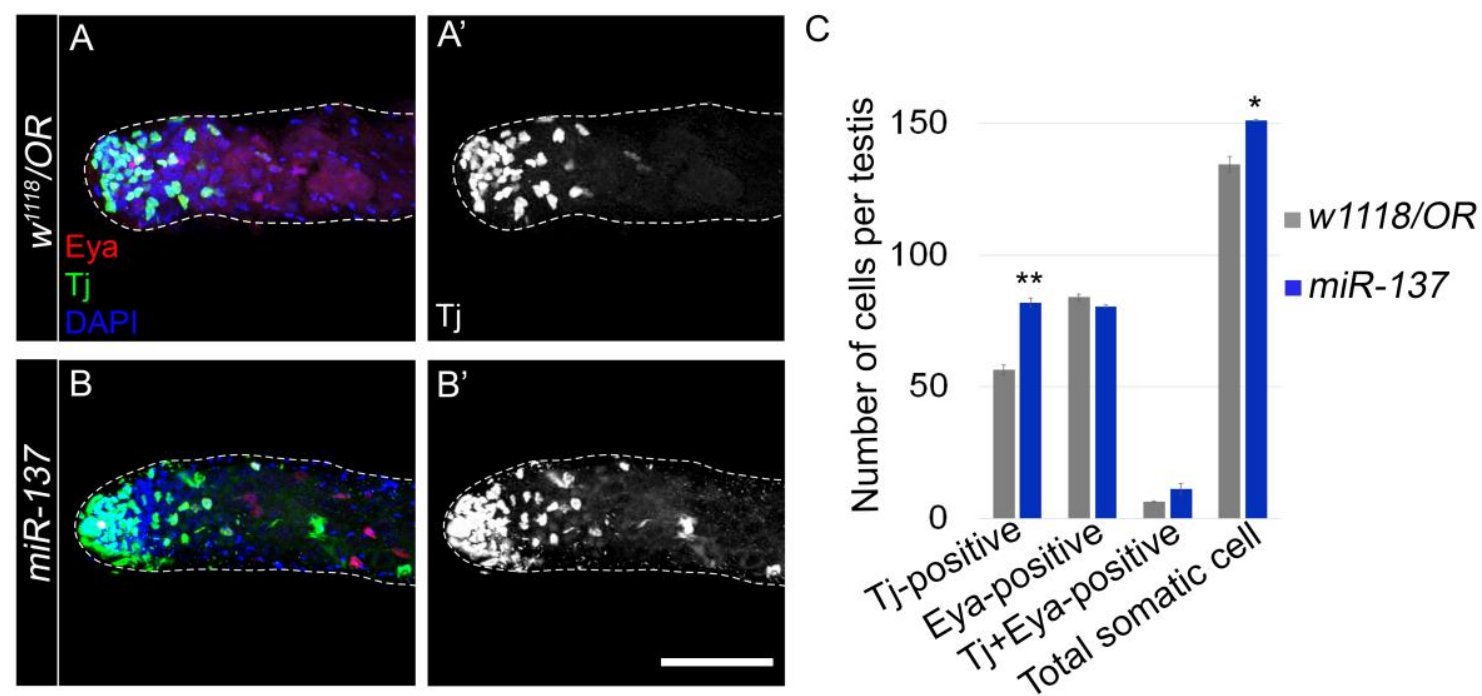

\section{Figure 20. MiR-137 $7^{k o}$ mutants have an increase in somatic cell population}

Representative images of the apex of control testis (A) and miR-137 testis (B) stained with early and late somatic cell markers $\mathrm{Tj}$ and Eya. In $m i R-137$ mutants, higher number of early somatic cells are visibly concentrated at the apex (B') compared to control (A'). (C) Quantification of the somatic cell population in control and miR-137 showing an increase in an early somatic cell population in $m i R-137$ resulting in a total increase in somatic cell count. No significant difference was observed in somatic cell numbers co-stained with anti- $\mathrm{Tj}$ and anti-Eya. Values are obtained from an average of 3 biological replicates. Error bars represent $\mathrm{AVE} \pm \mathrm{SEM}$ and statistical significance was determined by two-tailed Student's t-test. $* \mathrm{P}<0.05, * * \mathrm{P}<0.01, * * * \mathrm{P}<0.001$ Scale bar $50 \mu \mathrm{m}$. See also Supplementary Table 7.

Studies from the Drosophila ovary have shown that Dg is required to maintain epithelialcell polarity (Schneider et al., 2006) and is expressed in both germline and soma (Deng et al., 2003). In adult fly testes, Dg is also expressed in germline and soma (Figure 21). To further investigate the role of Dg in adult fly testes and to verify a miR-137- Dg interaction, we quantified the early somatic cell population in $m i R-137^{k o}(68.6 \pm 2.9)$ and $m i R-137^{k o / D f}$ $(36.7 \pm 2.9)$ flies. Furthermore, downregulation of $m i R-137$ specifically in early somatic cells by crossing tj-Gal4 with $U A S$-miR-137 $7^{\text {sponge }}$ causes an increase in the number of early somatic cells $(70.9 \pm 4.5)$, similar to the $m i R-137^{k o}$ phenotype. In addition, we also compared the downregulation of $m i R-137$ with the over-expression of $D g$ in early somatic cells and found that upon over-expression of $\mathrm{Dg}$, the early somatic counts were statistically irrelevant to controls $(42.2 \pm 1.5$ and $52.9 \pm 2.1$, respectively). $t j$-Gal4 lines used in this experiments were reported to be hypomorphic allele of $t j$, therefore, we suspected that the increased in early somatic cell counts in our controls $\left(\mathrm{tj} / \mathrm{w}^{1118}\right)$ is due to the reported mutation in the region of $t j$ transcription (Panchal et al., 2017). Further, we also over-expressed $m i R-137$ and scored 
for early somatic cell numbers and found no subsequent increase (50.2 \pm 0.9$)$ compared to controls. Similar quantification was also analyzed in lines that had downregulation of $m i R$ 137 in early somatic cells along with one copy loss of Dg in the background

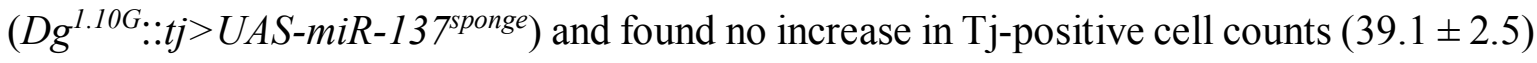
(Figure 21). From this experiment we concluded that $m i R-137$ regulates $D g$ to maintain early somatic cell population in fly testes, further indicating $D g$ is a relative target in testes.

We further verified the rescue of phenotype upon derepression of Dg in $m i R-137^{k o}$ by qRTPCR. RNA was extracted from 25-30 pairs of fly testes and normalized to control. We found increase in $D g$ mRNA levels in miR-137 $7^{k o}$ testes (4 fold increase) compared to $m i R-137^{k o / D f}$ ( 1.5 fold increase) relative to control, indicating the rescue of phenotype in $m i R-137^{k o / D f}$ was due to repression of $D g$ level. Consistent with these results, we found an increase in $D g$ mRNA level (2.5 fold) in $t j>U A S$-miR-137 $7^{\text {sponge }}$ mutants. When we remove one copy of $D g$ in this genetic background, we have only 1.5 fold increase in $D g$ levels. Our data suggest that $m i R-137$ can efficiently regulate $D g$ level in testes. Consistent with our in vitro data, we propose that $m i R-137$ is required to regulate $\mathrm{Dg}$ level to maintain early somatic cell numbers. A recent study has shown that knockdown of the soma-specific gene chic results in an increase in early somatic cell number and causes defective encapsulation of spermatocysts (Fairchild et al., 2015). We were further interested to know whether an increase in early somatic cell number leads to delayed differentiation that affects spermatogenesis in $m i R-137$ mutant testes. 

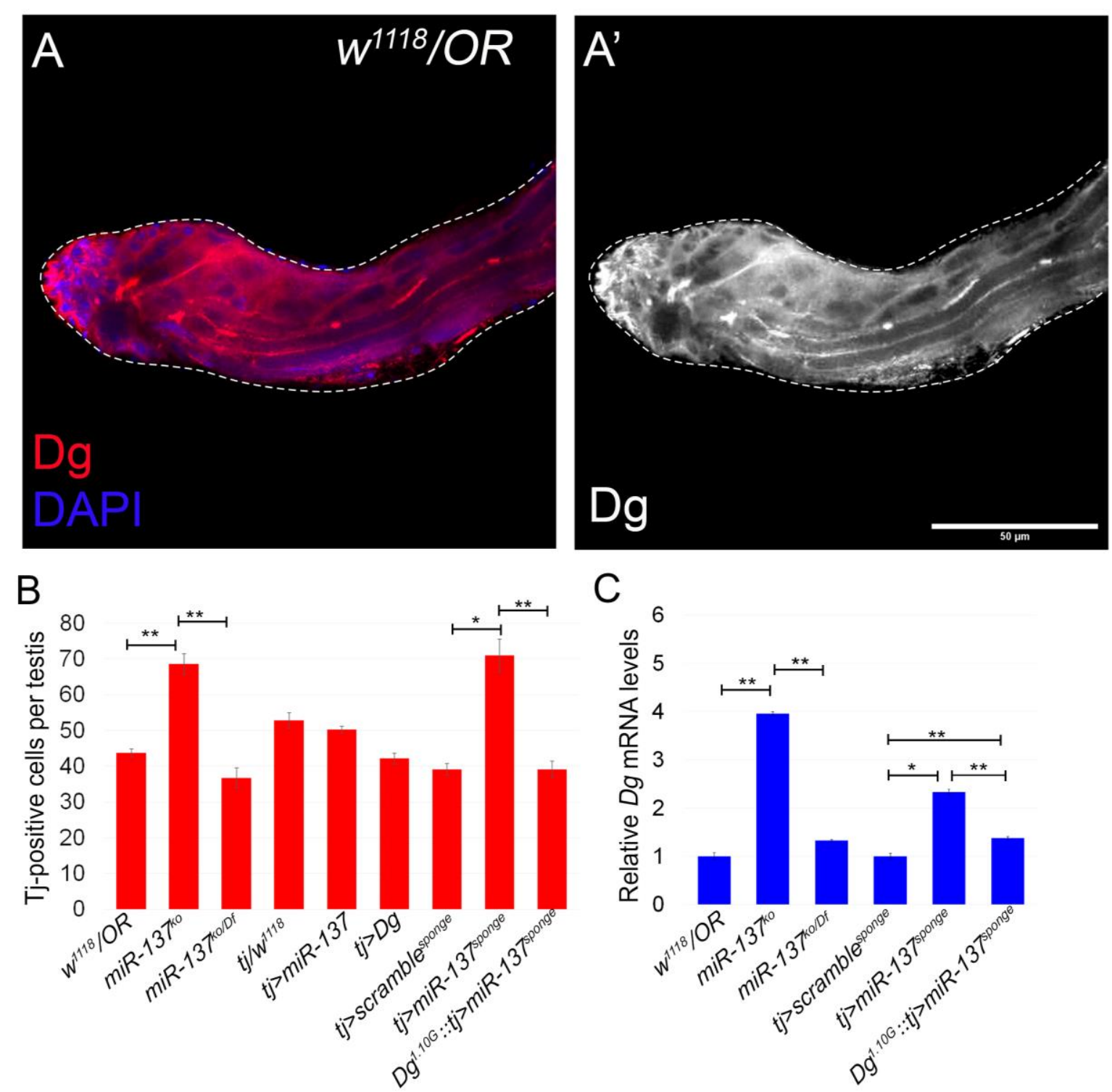

Figure 21. The early somatic cell population is maintained by downregulating Dg in

\section{miR-137 mutants}

(A) Dg staining in the apex of the control fly $\left(w^{1118} / O R\right)$ testis showing Dg is expressed in both soma and germline. (B) Quantification of the early somatic cell population in the apex of the adult fly testis shows an increase in early somatic cell numbers in $m i R-137^{k o}$ which was rescued in $m i R-137^{\mathrm{ko} / D f}$. No significant differences in early somatic cell population is detected upon over-expression of $D g$ as well as $m i R-137$. However, downregulation $m i R$ 137 in early somatic cells phenocopied the loss of function phenotype which is further rescued when one copy loss of Dg is maintained in the background. (C) A bar graph showing $D g$ mRNA levels in testes. Almost 4 fold increase in $D g$ mRNA level in $m i R-137^{k o}$, which is reduced to 1.5 fold in $m i R-137^{k o / D f}$. Similar observations are made with 2.5 fold increase upon down regulation of $m i R-137$ compared to 1.5 fold increase when one copy loss of $D g$ is maintained in the background. Values are obtained from averages of 2 biological replicates. Error bars represent $\mathrm{AVE} \pm \mathrm{SEM}$ and statistical significance was determined by two-tailed Student's t-test. ${ }^{*} \mathrm{P}<0.05,{ }^{*} \mathrm{P}<0.01, * * * \mathrm{P}<0.001$ Scale bar $50 \mu \mathrm{m}$. See also Supplementary Table 8 . 


\subsubsection{MiR-137 is essential to maintain permeability barrier}

Proper gametogenesis requires continuous interaction between soma and germline. Soma encapsulates germline ensuring cell integrity, supply with nutrients and signaling cues required for proper differentiation. Isolation of germline is maintained via a permeability barrier that is formed by soma. Previous research has reported that alteration in somatic cell differentiation perturbed this barrier. Since $m i R-137$ mutants have a higher number of early somatic cells and possibly abnormal differentiation (Figure 20), we were interested to know whether this affects the permeability barrier during spermatogenesis. Therefore, we performed a permeability assay with $10 \mathrm{kDa}$ Dextran dye. Permeability assay is a noninvasive assay that asses isolated germline from the outer environment surrounding somatic cells (Fairchild et al., 2015). After incubating the dissected testis in dye for $30 \mathrm{~min}$, we found that in control testis, dye was trapped around the barrier surrounding the 8- to 16-cell cyst, while in miR-137 mutants majority of the testes that were analyzed had dye detected inside the germline indicating defective blood-testis barrier (BTB). Almost 90\% of the analyzed testes were found to have defective BTB while only $28 \%$ of defective BTB was scored in control flies (Figure 22B).

Our previous data suggests that $D g$ is a bona fide target of $m i R-137$. To further investigate, whether miR-137-Dg interaction is also required to maintain $\mathrm{BTB}$, we performed the permeability assay in both $m i R-137^{k o / D f}$ and $D g$ mutant allele $\left(D g^{1.10 G}\right)$. We found that not only $D g^{1.10 G}$ has the perturbed BTB phenotype ( $86 \%$ defective BTB compared to control) but this phenotype was rescued in $m i R-137^{k o / D f}$ flies (38\% of defective BTB similar to $30 \%$ in control). Downregulation of $m i R-137$ in early somatic cell causes defective BTB (70\%) similar to over-expression of Dg. However, no effect was noted on BTB upon overexpression of $m i R-137$. Consistent to our previous data, it further indicated that $m i R-137$ repressed $D g$ to maintain BTB. To further verify our rescue due to $m i R-137-D g$ interaction, first, we analyzed over-expression of $m i R-137$ in early somatic cells with homozygous $m i R$ $137^{k o}$ background. Second, we analyzed downregulation of $m i R-137$ in early somatic cells with a heterozygous $D g^{1.10 G}$ allele in the background. In both cases, we observed an intact BTB, similar to controls. This is consistent with the results of the rescue experiments that demonstrates that downregulation of $\mathrm{Dg}$ in males that have miR-137 levels reduced specifically in early somatic cells, fully rescues the frequencies of the defective BTB phenotype. This further indicates, $m i R-137$ is required in early somatic cells to maintain the $\mathrm{BTB}$; in particular, $m i R-137$ is required to maintain the adequate level of ECM receptor $D g$ 
in early somatic cells to maintain proper formation of cellular junction, possibly septate junctions (SJs).
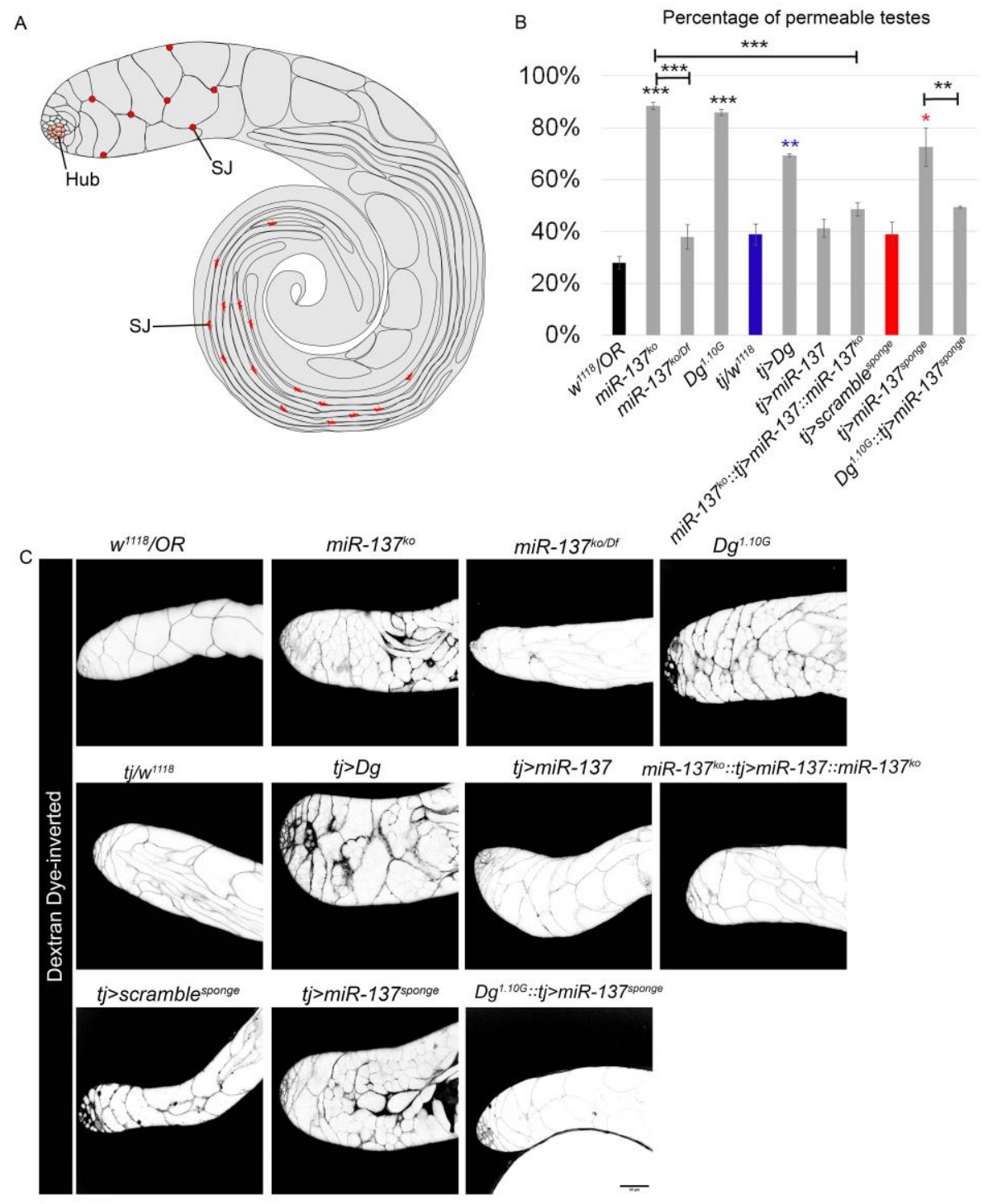

Figure 22. Permeability barrier is maintained by miR-137

(A) Schematic of differentiating gonialblasts encapsulated with two somatic cells forming spermatocyst in adult testes. The septate junction (SJ) are formed between two somatic cyst cells within the same cyst shown red in both early cyst cells (8- to 16-cell cyst) and in elongated spermatids. (B) Quantification of BTB phenotype in fly testes. $D g^{1.10 G}$, as well as over-expression of $\mathrm{Dg}$, shows perturbed BTB. MiR-137 loss-of-function and its downregulation in early somatic cells also have perturbed BTB which is rescued upon $m i R$ -

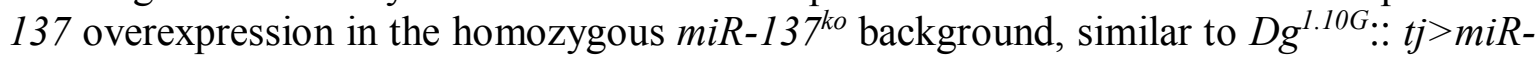
$137^{\text {sponge }}$, indicating downregulation of Dg level partially rescues the BTB phenotype. (C) Representative images of permeability assays showing loss of BTB in $m i R-137^{k o}, D g^{1.10 G}$, $t j>D g$, and $t j>m i R-137^{\text {sponge }}$. Values are obtained from averages of 3 biological replicates. Error bars represent $\mathrm{AVE} \pm \mathrm{SEM}$ and statistical significance was determined by two-tailed Student's t-test. ${ }^{*} \mathrm{P}<0.05, * * \mathrm{P}<0.01,{ }^{*} * \mathrm{P}<0.001$ Scale bar $50 \mu \mathrm{m}$. See also Supplementary Table 9. 


\subsubsection{MiR-137 mutants have abnormal septate junction (SJ) mor- phology}

The major septate junction (SJ) proteins, such as Nrx-IV, Cora and Dlg have been well characterized in establishing the permeability barrier (also known as blood-brain barrier i.e. BBB) in invertebrate brain (Hindle and Bainton, 2014). Studies have also shown that these proteins are localized around spermatocysts and knockdown of SJ proteins also leads to perturbed permeability barrier in fly testes (Fairchild et al., 2015). To further investigate the cause of an increase in perturbed permeability barrier in early spermatocysts in $m i R-137$ mutants, we further stained the adult fly testis with antibodies for SJ marker: anti-Dlg and anti-Mega and analyzed their morphology. In elongated spermatids, SJ proteins maintain the bridge between the two somatic cells appearing " $\mathrm{H}$ " or " $\mathrm{Z}$ " like structure (Figure 23 A-A', pointed by yellow arrows). When this morphology is compromised, the immune staining appeared more like a "dot" or a "dashed line" (Figure 23 A-A', pointed by white arrows). In miR-137 mutants, we found that approximately 50\% of SJ had abnormal morphology compared to $20 \%$ in control. A similar case had been observed upon downregulation of $m i R$ 137 in early somatic cells compared to the control with an average of $45 \%$ abnormal SJ compared to $15 \%$ in controls (Figure 23B). Further quantification on flies with genotypes: $t j>m i R-137$ and $m i R 137^{k o / D f}$ showed a decrease in number of abnormal SJ morphology (18\% and $30 \%$, respectively) compared to $50 \%$ of abnormal SJ in $m i R 137^{k o}$. Importantly, no significant change was observed in average number of SJ counted per testis in all animals (Supplementary Table 10), which suggests that the occurrence of SJ formation is normal in $m i R-137$ mutants, while the morphology of their SJs is abnormal. Statistical analysis was not performed on $t j>m i R-137$ and $m i R 137^{k o / D f}$ due to lack of biological replicates.

Altogether our data indicates that loss of $m i R-137$ can cause increase in early somatic cell numbers leading to defective SJ formation and defective BTB. Since our data also show that miR-137 is required for regulation of Dg levels in somatic cells in testis, it would be interesting to study further, if interaction between $m i R-137$ and $D g$ is also necessary for proper SJ morphology. 

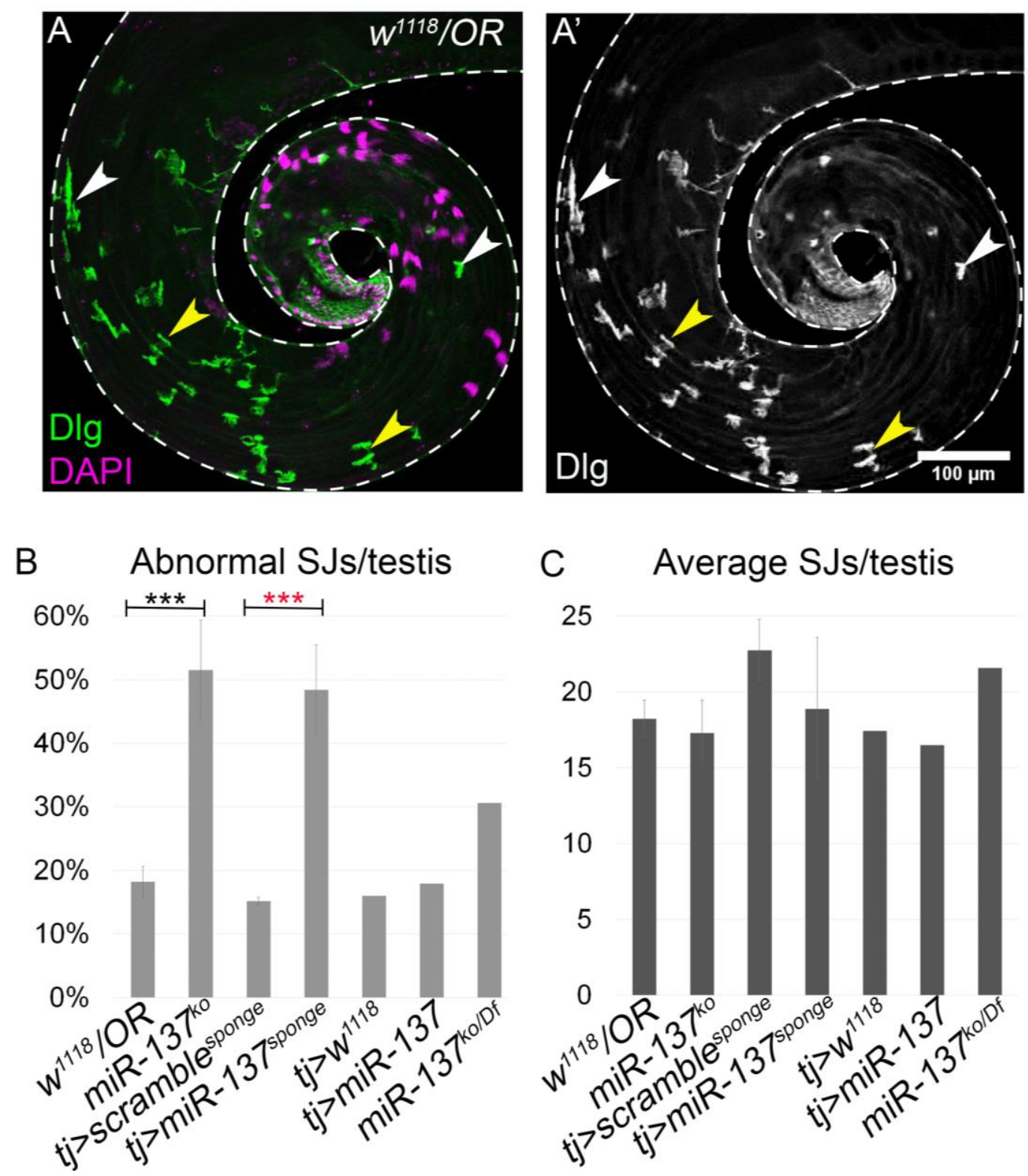

\section{Figure 23. MiR-137 mutants have defective septate junction phenotype}

(A) Septate junction protein Dlg staining in adult control (w1118/OR) testis showing a normal septate junction morphology in elongated spermatids (yellow arrowheads) and abnormal septate junction morphology (white arrowheads). (B) Quantification of abnormal septate junction morphology and (C) quantification of the average number of SJ count per testis. About $50 \%$ of the septate junction in miR-137 have abnormal morphology and no significant difference on the average numbers of SJ present in elongated spermatids per testis is found. Black star represents values obtained from averages of two biological replicates. Error bars represent $\mathrm{AVE} \pm \mathrm{SEM}$ and statistical significance was determined by two-tailed Student's t-test. $* \mathrm{P}<0.05, * * \mathrm{P}<0.01, * * * \mathrm{P}<0.001$. Red star represents statistical significance determined by $\chi^{2}$ test with Yate's correction $(* \mathrm{P}<0.05, * * \mathrm{P}<0.01, * * * \mathrm{P}<0.001)$. Scale bar $100 \mu \mathrm{m}$. See also Supplementary Table 10. 


\section{Discussion}

MDs are a group of diseases having diverse phenotypes related to neuronal and muscular systems in humans. MDs are genetically linked to the DGC and various animal models for the DGC have contributed significantly to understanding the variability of phenotypes in MDs, however, questions regarding the pathogenesis still remain unanswered.

The DGC involves a major transmembrane protein Dg that connects ECM to cytoskeleton accounting for the flexibility and durability of the plasma membrane providing signal transduction platform to many signaling molecules like Syn and nNOS. Hypoglycosylation of $\mathrm{Dg}$ is involved in various forms of dystroglycanopathies, CMD, and LGMD. In flies, studies have shown that loss of Dg cause muscle degeneration and the phenotype is enhanced upon stress (Kucherenko et al., 2011). Similarly, flies with Dg mutation are shown to have a low survival rate, delayed in development, altered metabolism, decrease mobility and agedependent muscle degeneration (Shcherbata et al., 2007). Reduced level of metabolic rate is also linked to miRNA expression (Biggar and Storey, 2011). MiRNAs are also implicated in the stabilization of diverse biological processes and to contribute to the consequences of normal development and physiological conditions and disease in different animals systems (Ebert and Sharp, 2012). MiRNAs having the ability to target multiple genes at once can regulate a complex network of genes influencing signaling required to maintain proper cellular homeostasis. Similarly, any imbalance in cellular homeostasis caused by stress or disease state can further influence miRNAs expression. Therefore, gene expression and miRNA levels are reciprocally regulated. The expression of miRNAs profile has been shown to be altered in Duchenne muscular dystrophy. The expression of certain miRNAs (miR-1, $m i R-133$, and $m i R-206$ ) have been shown to correlate with severity of the diseases and was proposed as diagnostic markers for DMD (Cacchiarelli et al., 2011b). In flies, Dg-Dys-Syn1 signaling has been reported to regulate miRNA profile (Marrone et al., 2012). Importantly, the study also showed that a similar level of miRNA profiles was altered upon stress in wild type animals indicating stress and MD share a common regulatory pathway to overcome negative effect in animal health. This study further reports the role of miRNAs in DGC signaling and their contribution to MDs and its pathogenesis in greater detail. 


\subsection{MiRNAs are required for muscle maintenance}

In this study, we have shown that the absence of miRNAs that are predicted to target multiple components of the DGC (Dg plus any three other components) affects muscle maintenance in flies. Dg was chosen as a baseline targeting component while grouping these miRNAs as Dg is associated with multiple pathways. Besides Dg-Dys-Syn1 pathway, it has been reported that Dg interacts with Hippo-signaling pathway component Yap to prevent cardiomyocyte proliferation (Morikawa et al., 2017). Inhibition of ubiquitinates ligase Trim32 has been shown to enhance plakoglobin binding to affect muscle atrophy (Cohen et al., 2014) and we further postulated that it can interact with Dg similar to plakoglobin to promote muscle maintenance. Therefore, miRNAs targeting the DGC importantly, Dg can influence more likely muscle maintenance phenotype associated with MDs. Further, Dg is required for correct localization of many other proteins such as Nrx-IV, Cora and GluRs mutations of which are also associated with MD-like phenotypes.

miRNAs are known to mediate stress response in animals to maintain cellular homeostasis. The expression of many of the miRNAs predicted to target the DGC components have been reported to be deregulated under hyperthermia stress in wild-type animals and/or in absence of Dg or Dys (Marrone et al., 2012). A prolong disease state can display immense stress for organismal health. Therefore, it was interesting to investigate miRNAs regulating a common pathway between stress and MD to decipher MD-related pathogenesis. This study, in particular, determines that in absence of $m i R-137, m i R-927$, and $m i R-966$ the muscle integrity in animals was severely compromised. This study further validates various stresses and aging can affect muscle degeneration even in control flies. Further, we were able to show that besides muscle degeneration, muscle atrophy is also increased in control animals during different stress conditions such as temperature stress, sugar starvation, and aging. All miRNA mutants were responsive to stress similar to control animals, indicating long term stress i.e. aging is more detrimental than a short term stresses such as hyperthermia stress and nutrition amelioration. Muscle atrophy was affected more than muscle degeneration in all genotypes in both stress and aging. This further highlights the importance of muscle maintenance in aging or in a prolonged disease state such as cancer that leads to cachexia. Not only miRNA loss was found to affect muscle integrity, but downregulation of Dg was also detrimental for healthy musculature. Previous studies have shown loss of Dg and Dys cause muscle degeneration and the phenotype is accelerated upon stress (Kucherenko et al., 2011). We further showed that upon downregulation of Dg specifically in adult muscle, 
cause muscle degeneration and atrophy in flies. Muscle atrophy was more detrimental as the frequency of muscle atrophy was higher than the frequency of muscle degeneration compared to control indicating, a proper level of $\mathrm{Dg}$ is required to maintain a healthy musculature in young and aged animals. Apart from its role in muscle maintenance, number of studies have reported that precise level of $\mathrm{Dg}$ is required to maintain energy homeostasis, establishment of NMJ, photoreceptor differentiation, and cellular polarity (Bogdanik et al., 2008; Marrone et al., 2011a; Shcherbata et al., 2007; Yatsenko et al., 2009). In flies, $m i R-9 a$ and $m i R-310 s$ had been shown previously to serve as a regulatory molecule to maintain the proper level of $\mathrm{Dg}$ to canalize myotendinous junction formation and to buffer MD related type II lissencephaly phenotype. $m i R-137, m i R-927$, and $m i R-966$ can further play a similar role in maintaining Dg levels in muscle to protect animals from stress- or age-related muscular dysfunction.

qRT-PCR on $m i R-137$ and $m i R-966$ mutants showed upregulated mRNA levels of either, Dys and/or Syn 1 even though they were not the predicted to target these genes while miR-927 mutants had either no change in mRNA levels or showed downregulation of $D g, D y s$, and Syn 1 even though it is predicted to target all three components. There can be a number of reasons following this discrepancy: 1) many miRNAs can have a tissue or sex-specific expression; since qRT-PCR was performed on the whole body of the organism, tissuespecific miRNA expression can be easily masked when normalized to whole body of control genotype. In the future, tissue-specific qRT-PCR could resolve this issue to validate the prediction based miRNA targeting in vivo. 2) The DGC signaling had been shown to have compensatory effects (Cote et al., 2002; Gao and McNally, 2015; Hughes et al., 2018), the fluctuation of mRNA levels between its predicted and non predicted targets could simply be the mimic of compensatory mechanisms of the DGC components.

MiR-137 and miR-966 mutants showed muscle degeneration as early as young age indicating, these miRNAs are required in muscle during development. The severity of muscle degeneration upon aging was more prominent for miR-966 mutants compared to temperature stress and sugar starvation. This further indicated that miR-966 is required to maintain healthy muscle in aging. MiR-966 is also reported to be enriched in hemolymph in old flies (Dhahbi et al., 2016). We have also shown from lifespan analysis that miR-966 mutants, in general, had a higher survival rate compared to control (Supplementary Figure 3). Altogether, these findings further suggest that loss of $m i R-966$ can be more responsive to physiological changes related to aging. Muscle atrophy was also observed in all miRNA 
mutants at a young age. This further indicates that all three miRNAs are required during developmental stage to maintain muscle integrity.

Muscle degeneration phenotype can be caused by muscles as well as neuronal defects. GO component term analyzed on conserved predicted mRNA targets of all three candidate miRNAs implied that these miRNA can be involved in the cell membrane and cell periphery related processes. MiR-137 having the most predicted targets, in addition, can be associated with neuronal processes like synapse and NMJs. GO terms for predicted miRNAs targets are in agreement with Dg functions. Luciferase reporter assay further confirmed that all three miRNAs $m i R-137, m i R-927$, and $m i R-966$ can downregulate $D g$ in vitro. Therefore, an in vivo screen on miRNAs gain-of-function was performed to further confirm all three selected miRNAs can downregulate Dg and/or any other muscle-specific target genes. As expected, over-expression of miRNAs gave stronger phenotypes as compared to their loss-of-function mutants. Over-expression of $m i R-137$ was the most severe in flies as its over-expression during developmental stage cause embryonic lethality, while over-expression in adult muscle resulted in strong muscle degeneration or complete absence of individual muscle. The experiment further confirmed that $m i R-927$ is required more during developing muscle as over-expression of miR-927 with how-Gal4 was lethal during the embryonic stage. Though the severity of phenotype can also depend on the strength of the UAS-miR-lines used, we observed that over-expression of miR-966 in adult muscle resulted in more severe phenotype compared to developing muscle. Over-expression in developing muscle also affected the muscle maintenance at young age but the effect of aging was prominent in flies. This further indicates, miR-966 can regulate age-dependent gene expression to maintain healthy muscles in animals. Many muscle-specific miRNAs (myomiRs) have been proposed as biomarkers for pathological and physiological muscle processes. myomiRs such as $m i R$ 1, $m i R-133, m i R-206, m i R-208$, and $m i R-499$ have been proposed as a diagnostic marker for DMD (Cacchiarelli et al., 2011b; Jeanson-Leh et al., 2014; Li et al., 2014). This study further suggests, $m i R-137$, $m i R-927$, and $m i R-966$ can act as a regulatory molecule to regulate $\mathrm{Dg}$ level in respons to stress to maintain healthy muscle in flies. 


\subsection{MiR-137 is required cell autonomously for muscle maintenance}

This study further deciphers the role of newly emerged miR-137 in MD development. MiR137 is highly conserved miRNA. Not only the seed sequence of miR-137 is conserved, but the mature miRNA itself is conserved from flies to humans. It is also predicted to target $D g$ and $S c g$, the major transmembrane components of the DGC in all higher eukaryotes. We further confirmed that $D g$ is the direct target of miR-137 in vitro. MiR-137 is a stressdependent miRNAs as its expression was downregulated upon hyperthermia stress in wildtype flies (Marrone et al., 2012). Dg mutants are known to have preference for low temperature $\left(18^{\circ} \mathrm{C}\right)$ (Takeuchi et al., 2009), and in microarray screen, miR-137 level remain unchanged in dystrophic flies at hyperthermia stress suggesting; 1) temperature fluctuation alone can cause a decreased level of $m i R-137$ in wild type flies and this downregulation relies on the DGC and 2) $D g$ mutants already have deregulated miR-137 and temperature stress doesn't affect its level on the DGC mutants. Compared to all the candidate miRNA mutants in our screen, the severity of phenotypes was observed higher in $m i R-137$ mutants at young and stress conditions, indicating it is required during developmental stage as well as for normal stress response. This was further confirmed by phenotypes observed upon downregulation of $m i R-137$ during developing and adult muscle. Regardless of the activity of the drivers used (Mhc-Gal4 and how-Gal4), muscle maintenance was severely compromised. Though the severity of phenotypes was observed stronger upon its downregulation in adult muscle compared to developing muscle, it was also noted that upon stress, the phenotypes were accelerated by more than two-fold when compared to young animals. This further confirmed, in adult muscle, $m i R-137$ is required to address the negative effect of stress. The over-expression of $m i R-137$ caused stronger phenotype leading to lethality and complete absence of muscle. Therefore, the study also confirms that $m i R-137$ levels must be maintained to have proper muscle function. Besides muscle maintenance phenotype, miR-137 mutant flies have climbing defects and increased variance in zygotic PGCs (Chen et al., 2014). In addition, they have a low survival rate (Supplementary Figure 2) and are developmentally delayed (data not shown). miR-137 is enriched in adult brain in mammals (He and Hannon, 2004) and is studied extensively in many retrospect involving schizophrenia, intellectual disability, neurodegeneration disorders such as Parkinson's Disease, synaptic development and dendritic arborization, and cancer profiling (Kong et al., 2015; Langevin et al., 2011; Ma et al., 2018; Munker and Calin, 2011; Olde Loohuis et al., 
2012; Silber et al., 2008; Smrt et al., 2010; Verma et al., 2015; Willemsen et al., 2011). In Drosophila, we were able to show its expression in neuronal and muscle tissues as well as in early somatic cell of adult fly testes. The previous study has also detected its expression in the adult fly thorax (Fulga et al., 2015). It will be interesting to further investigate the neuronal involvement of miR-137 to further explore its function in MDs or in other neurological disorders.

\subsection{MiR-137 is required to maintain a precise level of $\mathrm{Dg}$ in adult muscle}

We have shown that the level of Dg in adult muscle affects muscle integrity in age-dependent manner. To investigate if this effect can be ameliorated by over-expression of $\mathrm{Dg}$ in adult muscle, over-expression of Dg in adult muscle was analyzed at young, aged, and stressed animals. Surprisingly, it was found that not only its downregulation affects muscle maintenance, its over-expression also had a negative effect on normal muscle function. Muscle degeneration was seen in flies already at a young age, while muscle atrophy was not affected. This further indicated that over-expression of $\mathrm{Dg}$ is protective for flies to reduce atrophy upon aging. However, during temperature stress and sugar starvation, flies had more incidence of muscle degeneration and atrophy compared to control flies, indicating flies with over-expression of $\mathrm{Dg}$ can respond differently to different stress conditions. This study further implied that a proper level of $\mathrm{Dg}$ is required to maintain healthy adult muscle; therefore, it is interesting to investigate further how levels of Dg can affect muscle integrity in general.

To further investigate whether $m i R-137$ can regulate $\mathrm{Dg}$ level in muscle, $m i R-137$ mutants with one copy loss of $D g$ in the background were further analyzed. If $D g$ is a direct target in muscle, the level of Dg in $m i R-137^{\mathrm{ko} / D f}$ should be normalized to endogenous level, hence, the severity of phenotype should be reduced in $m i R-137^{k o / D f}$ lines compared to $m i R-137^{k o}$ lines. Analysis of IMFs of flies showed that in general, $m i R-137^{\mathrm{ko} / D f}$ flies had stronger phenotype compared to control, however, this was reduced almost by two times when compared to $m i R$ $137^{k o}$. Our data suggests that miR-137 regulates Dg levels and affects adult muscle maintenance. The difference in severity of phenotype was not visible in flies at a young age further suggesting a precise level of $\mathrm{Dg}$ is more important to overcome muscle loss upon stress. qRT-PCR performed on adult fly thoraces further confirmed relatively low $D g$ mRNA levels on $m i R-137^{k o / D f}$ lines compared to $m i R-137^{k o}$. Therefore, only partial rescue of muscle 
maintenance phenotype was observed during various stresses upon one copy loss of $D g$ in miR-137 mutants. At normal condition, over-expression, as well as loss of $\mathrm{Dg}$, posed a negative effect on muscles. A similar case was observed upon $m i R-137$ over-expression and loss-of-function. The down-regulation of $m i R-137$ was not as severe as over-expression of Dg and over-expression of $m i R-137$ was more severe than phenotypes reported for Dg lossof-function at ambient condition. Taken together, we propose that $m i R-137$ can target more muscle-specific genes other than $D g$ and influence MD development. Based on the prediction tool and qRT-PCR on miRNA mutants showed that miR-137 can further target Dys, Scg, NOS, Nrx-IV, and Mei-P26 as DGC components. It will be interesting to investigate in future how miR-137 can affect the expression of the DGC components to contribute to MD development.

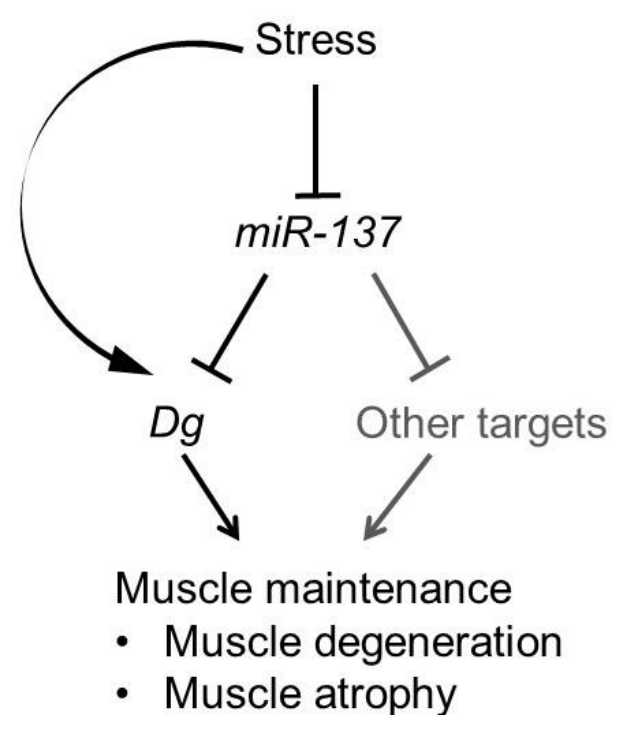

\section{Figure 24. Mode of action of $m i R-137$ in muscle}

During stress, $m i R-137$ is downregulated upon which its target mRNAs in muscle, including $D g$, are upregulated. This affects muscle maintenance such as muscle degeneration and muscle atrophy. Stress itself induces $\mathrm{Dg}$ expression that can further affect muscle maintenance. 


\subsection{MiR-137 is required in somatic cells to maintain permeability barrier}

This project further identifies the role of $m i R-137$ in Drosophila spermatogenesis. The in situ hybridization in wild type testis suggested that $m i R-137$ is possibly expressed in early somatic cells. Analysis of miR-137 mutant testes further showed an abnormal number of early somatic cell population at the apex of the testis (stained with anti-Tj) compared to control flies. We also observed similar phenotype upon miR-137 knock-down in early somatic cells. Our analysis showed that in male gametogenesis, miR-137 is required specifically in somatic cells. This was further supported by the total rescue of abnormal early somatic cell counts upon over-expression of $m i R-137$ in early somatic cells. The latter experiment also suggests that the endogenous level of $m i R-137$ can efficiently downregulate its target gene specifically in soma. The expansion of Tj-positive cells can be either due to an increase in proliferation or delayed in differentiation. However, we did not observe an abnormal number of CySCs stained with anti-PH3 (mitotic cell marker) in control and mutant testes. Further staining with anti-Add (spectrosome and fusome marker) showed an increase in differentiating germline cells in mutant testes supporting our latter hypothesis of delayed differentiation in miR-137 mutants. Though both the possibilities are not conclusive from our experiments, further staining with Edu together with anti-Zfh1 (a marker for CySCs and its early daughter cells) or staining with cell cycle marker like anti-Cyclin can further shed lights to the pinpoint increase in somatic proliferation or differentiation process.

We have shown from our data from fly muscle that $D g$ is a relative target of $m i R-137$. We have further shown that Dg is also expressed in germline and soma in fly testis. To further investigate whether the interaction between $m i R-137-D g$ is also required to establish cellular homeostasis during male gametogenesis, we analyzed the effect on early somatic cell numbers on $m i R-137^{k o / D f}$ lines first. We did not find abnormal early somatic cell numbers in $m i R-137^{k o / D f}$ testes. The reduced level of Dg in $m i R-137^{k o}$ lines fully rescued the abnormal somatic cell number phenotype. A similar observation was made on genetic rescue achieved

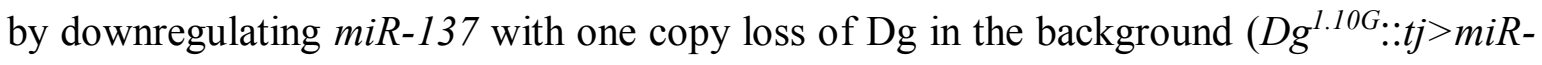
$\left.137^{\text {sponge }}\right)$. A significant difference in early somatic cells population as observed in genetic rescue compared to down-regulation of $m i R-137$ further supports the idea that $m i R-137$ is required in early somatic cells in adult fly testis and regulates the Dg level in order to maintain proper spermatogenesis. Therefore, in testis, $\mathrm{Dg}$ is the relative target of $m i R-137$. 


\subsection{Dg levels must be regulated to maintain the permeability barrier}

In male gametogenesis, somatic cells are required to encapsulate differentiating germlines and maintain a barrier around them. Each spermatocyst (germline enveloped with two somatic cells) is connected with neighboring spermatocysts by their outer somatic membrane maintaining a closed barrier between inner-membrane of the somatic cell and the germline. This barrier is also known as the permeability or blood-testis barrier (BTB). Genes required in somatic cells in Drosophila testis have been shown to have an important function in spermatogenesis (Fairchild et al., 2017). Knockdown of the soma-specific gene (chic) has been shown to regulate cell proliferation and affect the permeability barrier (Fairchild et al., 2015). Consistent with this study, we also found that $m i R-137$ mutants have perturbed BTB and this phenotype was rescued upon one copy loss of $D g$ in $m i R-137$ mutants. Our data further verified the increase in early somatic cells can cause perturbed BTB. Interestingly, both loss and over-expression of Dg caused perturbed BTB. Knock-down of $m i R-137$ in early somatic cell recapitulated the BTB phenotype of Dg over-expression in early somatic cells and this phenotype was rescued upon downregulation of $m i R-137$ with one copy loss of $\mathrm{Dg}$ in the background as well as with over-expression of $m i R-137$ in homozygous $m i R$ $137^{k o}$ background ( $\left.m i R-137^{k o}:: t j>m i R-137:: m i R-137^{k o}\right)$. Taken together, these results show that $m i R-137$ is required to regulate $\mathrm{Dg}$ level in soma to maintain permeability barrier. This study further claims the scope of perturbed BTB as a MD related phenotype and miRNAs are required to regulate the level of the DGC components in Drosophila spermatogenesis.

\subsection{MiR-137 acts in soma to regulate Dg in septate junctions}

Studies have shown that the permeability barrier is dependent on the function of septate junction proteins. Septate junction (SJ) proteins like Cora and Nrx-IV have been shown to be concentrated at the sites of contact between two somatic cells that encapsulates the germline (Fairchild et al., 2015). The study also reported that knock-down of these components cause the BTB phenotype. Recent progression made on understanding Drosophila spermatogenesis also showed that loss of Dg cause mislocalization of SJ proteins Cora and Nrx-IV resulting in perturbed BTB (unpublished data). The study also showed that downregulation of Dg in soma, as well as germline, caused perturbed BTB, indicating Dg is required in both soma and germlines to maintain BTB. Our study further showed that overexpression of Dg in somatic cells and its loss caused perturbed BTB. And this phenotype 
was recovered upon reducing the level of $D g$ in $m i R-137^{k o}$ flies. The observed phenotype of perturbed BTB was rescued not only in $m i R-137^{k o / D f}$ lines but also in $m i R-137^{k o}:: t j>m i R$ -

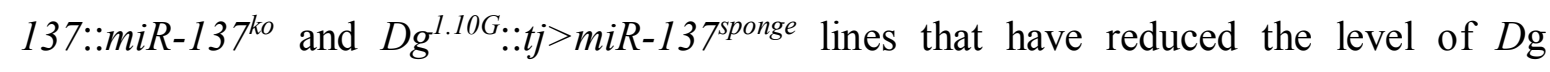
compared to $m i R-137^{k o}$. Our qRT-PCR data further showed that $D g$ mRNA levels in these genetic backgrounds varied vastly. Complete loss of miR-137 and its downregulation on early somatic cells caused an increase in $D g$ mRNA levels which was reduced by two-fold in $m i R-137^{\mathrm{ko} / D f}$ and one copy loss of $D g$ in $t j>m i R-137^{\text {sponge }}$. This further concluded that $m i R$ 137 regulates $D g$ level in soma and maintains the BTB.

Many SJ related proteins and genes that are required in soma are predicted targets of $m i R$ 137. Such protein includes Nrx-IV, Dlg, and Chic. It will be further interesting to study how $m i R-137$ influence the expression and their possible interaction with $D g$ to maintain spermatogenesis in Drosophila testis.

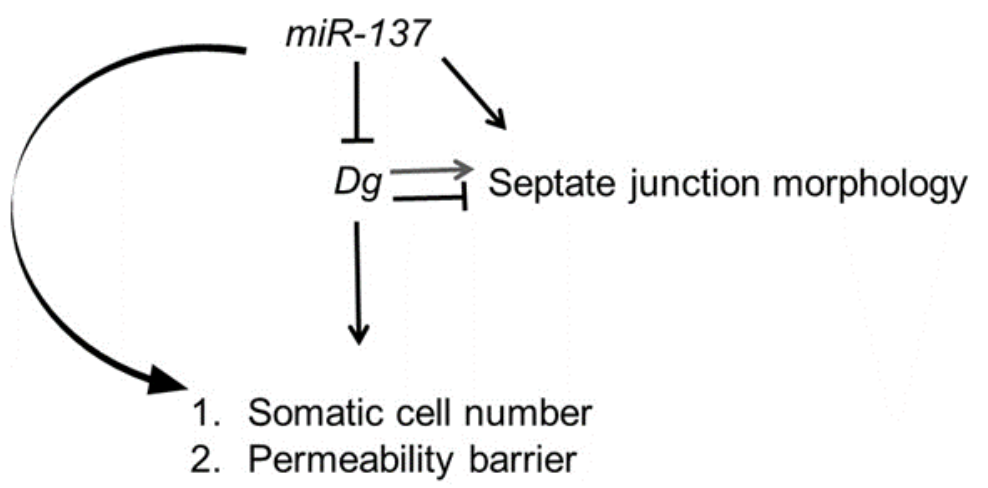

\section{Figure 25. Mode of action of $m i R-137$ in testis}

Loss of $m i R-137$ caused upregulation of $D g$ that increases somatic cell population and affect BTB. $m i R-137$ also affects septate junction morphology and it has been shown that upon Dg loss core components of septate junction protein are mislocalized. The possible interaction of $m i R-137$ and $D g$ to maintain septate junction morphology is yet to be defined (grey arrow). 


\section{Conclusions}

During this study, we identified a set of miRNAs that regulate muscle maintenance upon various stresses. Furthermore, our study demonstrates a novel role for miRNAs miR-137, $m i R-927$ and $m i R-966$ in regulation of major DGC components including Dg. We propose that these miRNA can be involved in fine-tuning of DGC signaling and MD development. In this study, a highly conserved miR-137 was found to target $D g$ 3'UTR and regulate its levels specifically in muscles and in testicular soma. Deregulation of $m i R-137$ and $\mathrm{Dg}$ expression lead to stress dependent muscle degeneration and atrophy. Therefore, we propose that $m i R-137$ potentially can be used as a biomarker and a candidate for MD therapeutics. Moreover, we found that miR-137 is required for proper blood-testis barrier (BTB) establishment which also requires proper levels of the ECM receptor Dg. Since, MDs in general, have multiple phenotypic characteristics in patients which show abnormalities not only in muscle but other systems, this study further implies that perturbed spermatogenesis could be a novel MD-related phenotype. 


\section{References}

Adams, M.E., Odom, G.L., Kim, M.J., Chamberlain, J.S., and Froehner, S.C. (2018). Syntrophin binds directly to multiple spectrin-like repeats in dystrophin and mediates binding of nNOS to repeats 16-17. Human molecular genetics 27, 2978-2985.

Ameres, S.L., Horwich, M.D., Hung, J.H., Xu, J., Ghildiyal, M., Weng, Z., and Zamore, P.D. (2010). Target RNA-directed trimming and tailing of small silencing RNAs. Science (New York, NY) 328, 1534-1539.

Astrea, G., Romano, A., Angelini, C., Antozzi, C.G., Barresi, R., Battini, R., Battisti, C., Bertini, E., Bruno, C., Cassandrini, D., et al. (2018). Broad phenotypic spectrum and genotype-phenotype correlations in GMPPB-related dystroglycanopathies: an Italian crosssectional study. Orphanet journal of rare diseases 13, 170.

Baccarini, A., Chauhan, H., Gardner, T.J., Jayaprakash, A.D., Sachidanandam, R., and Brown, B.D. (2011). Kinetic analysis reveals the fate of a microRNA following target regulation in mammalian cells. Current biology : CB 21, 369-376.

Baek, D., Villen, J., Shin, C., Camargo, F.D., Gygi, S.P., and Bartel, D.P. (2008). The impact of microRNAs on protein output. Nature 455, 64-71.

Balci, B., Uyanik, G., Dincer, P., Gross, C., Willer, T., Talim, B., Haliloglu, G., Kale, G., Hehr, U., Winkler, J., et al. (2005). An autosomal recessive limb girdle muscular dystrophy (LGMD2) with mild mental retardation is allelic to Walker-Warburg syndrome (WWS) caused by a mutation in the POMT1 gene. Neuromuscular disorders : NMD 15, 271-275.

Barman, B., and Bhattacharyya, S.N. (2015). mRNA Targeting to Endoplasmic Reticulum Precedes Ago Protein Interaction and MicroRNA (miRNA)-mediated Translation Repression in Mammalian Cells. The Journal of biological chemistry 290, 24650-24656.

Bartel, D.P. (2004). MicroRNAs: genomics, biogenesis, mechanism, and function. Cell 116, 281-297.

Bashir, R., Britton, S., Strachan, T., Keers, S., Vafiadaki, E., Lako, M., Richard, I., Marchand, S., Bourg, N., Argov, Z., et al. (1998). A gene related to Caenorhabditis elegans spermatogenesis factor fer- 1 is mutated in limb-girdle muscular dystrophy type $2 \mathrm{~B}$. Nature genetics 20,37-42.

Bazzini, A.A., Lee, M.T., and Giraldez, A.J. (2012). Ribosome profiling shows that miR430 reduces translation before causing mRNA decay in zebrafish. Science (New York, NY) 336, 233-237.

Behm-Ansmant, I., Rehwinkel, J., Doerks, T., Stark, A., Bork, P., and Izaurralde, E. (2006). mRNA degradation by miRNAs and GW182 requires both CCR4:NOT deadenylase and DCP1:DCP2 decapping complexes. Genes \& development 20, 1885-1898.

Bhat, H.F., Adams, M.E., and Khanday, F.A. (2013). Syntrophin proteins as Santa Claus: role(s) in cell signal transduction. Cellular and molecular life sciences : CMLS 70, 25332554. 
Biggar, K.K., and Storey, K.B. (2011). The emerging roles of microRNAs in the molecular responses of metabolic rate depression. Journal of molecular cell biology 3, 167-175.

Bogdanik, L., Framery, B., Frolich, A., Franco, B., Mornet, D., Bockaert, J., Sigrist, S.J., Grau, Y., and Parmentier, M.L. (2008). Muscle dystroglycan organizes the postsynapse and regulates presynaptic neurotransmitter release at the Drosophila neuromuscular junction. PloS one 3, e2084.

Bonnemann, C.G., Wang, C.H., Quijano-Roy, S., Deconinck, N., Bertini, E., Ferreiro, A., Muntoni, F., Sewry, C., Beroud, C., Mathews, K.D., et al. (2014). Diagnostic approach to the congenital muscular dystrophies. Neuromuscular disorders : NMD 24, 289-311.

Bose, M., Barman, B., Goswami, A., and Bhattacharyya, S.N. (2017). Spatiotemporal Uncoupling of MicroRNA-Mediated Translational Repression and Target RNA Degradation Controls MicroRNP Recycling in Mammalian Cells. Molecular and cellular biology 37.

Boyle, E.I., Weng, S., Gollub, J., Jin, H., Botstein, D., Cherry, J.M., and Sherlock, G. (2004). GO::TermFinder--open source software for accessing Gene Ontology information and finding significantly enriched Gene Ontology terms associated with a list of genes. Bioinformatics (Oxford, England) 20, 3710-3715.

Brand, A.H., and Perrimon, N. (1993). Targeted gene expression as a means of altering cell fates and generating dominant phenotypes. Development (Cambridge, England) 118, 401415.

Brennecke, J., Stark, A., Russell, R.B., and Cohen, S.M. (2005). Principles of microRNAtarget recognition. PLoS biology 3 , e85.

Broughton, J.P., Lovci, M.T., Huang, J.L., Yeo, G.W., and Pasquinelli, A.E. (2016). Pairing beyond the Seed Supports MicroRNA Targeting Specificity. Molecular cell 64, 320-333.

Brown, S.C., and Winder, S.J. (2017). 220th ENMC workshop: Dystroglycan and the dystroglycanopathies Naarden, The Netherlands, 27-29 May 2016. Neuromuscular disorders : NMD 27, 387-395.

Bryantsev, A.L., Duong, S., Brunetti, T.M., Chechenova, M.B., Lovato, T.L., Nelson, C., Shaw, E., Uhl, J.D., Gebelein, B., and Cripps, R.M. (2012). Extradenticle and homothorax control adult muscle fiber identity in Drosophila. Developmental cell 23, 664-673.

Bushati, N., and Cohen, S.M. (2007). microRNA functions. Annual review of cell and developmental biology 23, 175-205.

Cacchiarelli, D., Incitti, T., Martone, J., Cesana, M., Cazzella, V., Santini, T., Sthandier, O., and Bozzoni, I. (2011a). miR-31 modulates dystrophin expression: new implications for Duchenne muscular dystrophy therapy. EMBO reports 12, 136-141.

Cacchiarelli, D., Legnini, I., Martone, J., Cazzella, V., D'Amico, A., Bertini, E., and Bozzoni, I. (2011b). miRNAs as serum biomarkers for Duchenne muscular dystrophy. EMBO molecular medicine 3, 258-265. 
Cacchiarelli, D., Martone, J., Girardi, E., Cesana, M., Incitti, T., Morlando, M., Nicoletti, C., Santini, T., Sthandier, O., Barberi, L., et al. (2010). MicroRNAs involved in molecular circuitries relevant for the Duchenne muscular dystrophy pathogenesis are controlled by the dystrophin/nNOS pathway. Cell metabolism 12, 341-351.

Chaturvedi, D., Reichert, H., Gunage, R.D., and VijayRaghavan, K. (2017). Identification and functional characterization of muscle satellite cells in Drosophila. eLife 6.

Chen, J.F., Mandel, E.M., Thomson, J.M., Wu, Q., Callis, T.E., Hammond, S.M., Conlon, F.L., and Wang, D.Z. (2006). The role of microRNA-1 and microRNA-133 in skeletal muscle proliferation and differentiation. Nature genetics 38, 228-233.

Chen, K., and Rajewsky, N. (2007). The evolution of gene regulation by transcription factors and microRNAs. Nature reviews Genetics 8, 93-103.

Chen, Y.W., Song, S., Weng, R., Verma, P., Kugler, J.M., Buescher, M., Rouam, S., and Cohen, S.M. (2014). Systematic study of Drosophila microRNA functions using a collection of targeted knockout mutations. Developmental cell 31, 784-800.

Cheng, C.Y., and Mruk, D.D. (2012). The blood-testis barrier and its implications for male contraception. Pharmacological reviews 64, 16-64.

Choi, W.Y., Giraldez, A.J., and Schier, A.F. (2007). Target protectors reveal dampening and balancing of Nodal agonist and antagonist by miR-430. Science (New York, NY) 318, 271274.

Cicek, I.O., Karaca, S., Brankatschk, M., Eaton, S., Urlaub, H., and Shcherbata, H.R. (2016). Hedgehog Signaling Strength Is Orchestrated by the mir-310 Cluster of MicroRNAs in Response to Diet. Genetics 202, 1167-1183.

Cohen, S., Lee, D., Zhai, B., Gygi, S.P., and Goldberg, A.L. (2014). Trim32 reduces PI3KAkt-FoxO signaling in muscle atrophy by promoting plakoglobin-PI3K dissociation. The Journal of cell biology 204, 747-758.

Cohen, S.M. (2009). Use of microRNA sponges to explore tissue-specific microRNA functions in vivo. Nature methods $6,873-874$.

Cohn, R.D. (2005). Dystroglycan: important player in skeletal muscle and beyond. Neuromuscular disorders : NMD 15, 207-217.

Cooley, L., Kelley, R., and Spradling, A. (1988). Insertional mutagenesis of the Drosophila genome with single P elements. Science (New York, NY) 239, 1121-1128.

Cote, P.D., Moukhles, H., and Carbonetto, S. (2002). Dystroglycan is not required for localization of dystrophin, syntrophin, and neuronal nitric-oxide synthase at the sarcolemma but regulates integrin alpha 7B expression and caveolin-3 distribution. The Journal of biological chemistry $277,4672-4679$.

De Arcangelis, V., Serra, F., Cogoni, C., Vivarelli, E., Monaco, L., and Naro, F. (2010). beta1-syntrophin modulation by miR-222 in mdx mice. PloS one 5 . 
de Pontual, L., Yao, E., Callier, P., Faivre, L., Drouin, V., Cariou, S., Van Haeringen, A., Genevieve, D., Goldenberg, A., Oufadem, M., et al. (2011). Germline deletion of the miR17 approximately 92 cluster causes skeletal and growth defects in humans. Nature genetics 43, 1026-1030.

Demarco, R.S., Eikenes, A.H., Haglund, K., and Jones, D.L. (2014). Investigating spermatogenesis in Drosophila melanogaster. Methods (San Diego, Calif) 68, 218-227.

Demontis, F., Piccirillo, R., Goldberg, A.L., and Perrimon, N. (2013). Mechanisms of skeletal muscle aging: insights from Drosophila and mammalian models. Disease models \& mechanisms 6, 1339-1352.

Deng, W.M., Schneider, M., Frock, R., Castillejo-Lopez, C., Gaman, E.A., Baumgartner, S., and Ruohola-Baker, H. (2003). Dystroglycan is required for polarizing the epithelial cells and the oocyte in Drosophila. Development (Cambridge, England) 130, 173-184.

Denli, A.M., Tops, B.B., Plasterk, R.H., Ketting, R.F., and Hannon, G.J. (2004). Processing of primary microRNAs by the Microprocessor complex. Nature 432, 231-235.

Dhahbi, J.M. (2014). Circulating small noncoding RNAs as biomarkers of aging. Ageing research reviews $17,86-98$.

Dhahbi, J.M., Atamna, H., Li, R., Yamakawa, A., Guerrero, N., Lam, H.T., Mote, P., and Spindler, S.R. (2016). MicroRNAs Circulate in the Hemolymph of Drosophila and Accumulate Relative to Tissue microRNAs in an Age-Dependent Manner. Genomics insights 9, 29-39.

Djuranovic, S., Nahvi, A., and Green, R. (2012). miRNA-mediated gene silencing by translational repression followed by mRNA deadenylation and decay. Science (New York, NY) 336, 237-240.

Doench, J.G., and Sharp, P.A. (2004). Specificity of microRNA target selection in translational repression. Genes \& development 18, 504-511.

Durbeej, M., and Campbell, K.P. (2002). Muscular dystrophies involving the dystrophinglycoprotein complex: an overview of current mouse models. Current opinion in genetics \& development 12, 349-361.

Dutta, D., Anant, S., Ruiz-Gomez, M., Bate, M., and VijayRaghavan, K. (2004). Founder myoblasts and fibre number during adult myogenesis in Drosophila. Development (Cambridge, England) 131, 3761-3772.

Ebert, M.S., and Sharp, P.A. (2012). Roles for microRNAs in conferring robustness to biological processes. Cell 149, 515-524.

Edeleva, E.V., and Shcherbata, H.R. (2013). Stress-induced ECM alteration modulates cellular microRNAs that feedback to readjust the extracellular environment and cell behavior. Frontiers in genetics 4, 305 . 
Eisenberg, I., Eran, A., Nishino, I., Moggio, M., Lamperti, C., Amato, A.A., Lidov, H.G., Kang, P.B., North, K.N., Mitrani-Rosenbaum, S., et al. (2007). Distinctive patterns of microRNA expression in primary muscular disorders. Proceedings of the National Academy of Sciences of the United States of America 104, 17016-17021.

Enright, A.J., John, B., Gaul, U., Tuschl, T., Sander, C., and Marks, D.S. (2003). MicroRNA targets in Drosophila. Genome biology 5, R1.

Ervasti, J.M., and Campbell, K.P. (1993). Dystrophin-associated glycoproteins: their possible roles in the pathogenesis of Duchenne muscular dystrophy. Molecular and cell biology of human diseases series 3, 139-166.

Fairchild, M.J., Islam, F., and Tanentzapf, G. (2017). Identification of genetic networks that act in the somatic cells of the testis to mediate the developmental program of spermatogenesis. PLoS genetics 13, e1007026.

Fairchild, M.J., Smendziuk, C.M., and Tanentzapf, G. (2015). A somatic permeability barrier around the germline is essential for Drosophila spermatogenesis. Development (Cambridge, England) $142,268-281$.

Franca, L.R., Auharek, S.A., Hess, R.A., Dufour, J.M., and Hinton, B.T. (2012). Bloodtissue barriers: morphofunctional and immunological aspects of the blood-testis and bloodepididymal barriers. Advances in experimental medicine and biology 763, 237-259.

Frosk, P., Weiler, T., Nylen, E., Sudha, T., Greenberg, C.R., Morgan, K., Fujiwara, T.M., and Wrogemann, K. (2002). Limb-girdle muscular dystrophy type $2 \mathrm{H}$ associated with mutation in TRIM32, a putative E3-ubiquitin-ligase gene. American journal of human genetics 70, 663-672.

Fulga, T.A., McNeill, E.M., Binari, R., Yelick, J., Blanche, A., Booker, M., Steinkraus, B.R., Schnall-Levin, M., Zhao, Y., DeLuca, T., et al. (2015). A transgenic resource for conditional competitive inhibition of conserved Drosophila microRNAs. Nature communications 6, 7279 .

Gao, Q.Q., and McNally, E.M. (2015). The Dystrophin Complex: Structure, Function, and Implications for Therapy. Comprehensive Physiology 5, 1223-1239.

Gibbings, D.J., Ciaudo, C., Erhardt, M., and Voinnet, O. (2009). Multivesicular bodies associate with components of miRNA effector complexes and modulate miRNA activity. Nature cell biology 11, 1143-1149.

Grady, R.M., Zhou, H., Cunningham, J.M., Henry, M.D., Campbell, K.P., and Sanes, J.R. (2000). Maturation and maintenance of the neuromuscular synapse: genetic evidence for roles of the dystrophin--glycoprotein complex. Neuron 25, 279-293.

Greco, S., De Simone, M., Colussi, C., Zaccagnini, G., Fasanaro, P., Pescatori, M., Cardani, R., Perbellini, R., Isaia, E., Sale, P., et al. (2009). Common micro-RNA signature in skeletal muscle damage and regeneration induced by Duchenne muscular dystrophy and acute ischemia. FASEB journal : official publication of the Federation of American Societies for Experimental Biology 23, 3335-3346. 
Greener, M.J., and Roberts, R.G. (2000). Conservation of components of the dystrophin complex in Drosophila. FEBS letters 482, 13-18.

Grimson, A., Srivastava, M., Fahey, B., Woodcroft, B.J., Chiang, H.R., King, N., Degnan, B.M., Rokhsar, D.S., and Bartel, D.P. (2008). Early origins and evolution of microRNAs and Piwi-interacting RNAs in animals. Nature 455, 1193-1197.

Gunage, R.D., Dhanyasi, N., Reichert, H., and VijayRaghavan, K. (2017). Drosophila adult muscle development and regeneration. Seminars in cell \& developmental biology 72, 56-66. Guo, H., Ingolia, N.T., Weissman, J.S., and Bartel, D.P. (2010). Mammalian microRNAs predominantly act to decrease target mRNA levels. Nature $466,835-840$.

Guo, L., and Lu, Z. (2010). The Fate of miRNA* Strand through Evolutionary Analysis: Implication for Degradation As Merely Carrier Strand or Potential Regulatory Molecule? PloS one 5.

Ha, M., and Kim, V.N. (2014). Regulation of microRNA biogenesis. Nature reviews Molecular cell biology 15, 509-524.

Hardy, R.W., Tokuyasu, K.T., Lindsley, D.L., and Garavito, M. (1979). The germinal proliferation center in the testis of Drosophila melanogaster. Journal of ultrastructure research $69,180-190$.

He, L., and Hannon, G.J. (2004). MicroRNAs: small RNAs with a big role in gene regulation. Nature reviews Genetics 5, 522-531.

Heimberg, A.M., Sempere, L.F., Moy, V.N., Donoghue, P.C., and Peterson, K.J. (2008). MicroRNAs and the advent of vertebrate morphological complexity. Proceedings of the National Academy of Sciences of the United States of America 105, 2946-2950.

Herranz, H., Hong, X., Perez, L., Ferreira, A., Olivieri, D., Cohen, S.M., and Milan, M. (2010). The miRNA machinery targets Mei-P26 and regulates Myc protein levels in the Drosophila wing. The EMBO journal 29, 1688-1698.

Hindle, S.J., and Bainton, R.J. (2014). Barrier mechanisms in the Drosophila blood-brain barrier. Frontiers in neuroscience $8,414$.

Hoffman, E.P., Brown, R.H., Jr., and Kunkel, L.M. (1987). Dystrophin: the protein product of the Duchenne muscular dystrophy locus. Cell 51, 919-928.

Hornstein, E., Mansfield, J.H., Yekta, S., Hu, J.K., Harfe, B.D., McManus, M.T., Baskerville, S., Bartel, D.P., and Tabin, C.J. (2005). The microRNA miR-196 acts upstream of Hoxb8 and Shh in limb development. Nature 438, 671-674.

Hu, Y., Flockhart, I., Vinayagam, A., Bergwitz, C., Berger, B., Perrimon, N., and Mohr, S.E. (2011). An integrative approach to ortholog prediction for disease-focused and other functional studies. BMC bioinformatics 12, 357 .

Hughes, D.C., Marcotte, G.R., Baehr, L.M., West, D.W.D., Marshall, A.G., Ebert, S.M., Davidyan, A., Adams, C.M., Bodine, S.C., and Baar, K. (2018). Alterations in the muscle 
force transfer apparatus in aged rats during unloading and reloading: impact of microRNA31. The Journal of physiology 596, 2883-2900.

Jeanson-Leh, L., Lameth, J., Krimi, S., Buisset, J., Amor, F., Le Guiner, C., Barthelemy, I., Servais, L., Blot, S., Voit, T., et al. (2014). Serum profiling identifies novel muscle miRNA and cardiomyopathy-related miRNA biomarkers in Golden Retriever muscular dystrophy dogs and Duchenne muscular dystrophy patients. The American journal of pathology 184, 2885-2898.

Jiang, F., Ye, X., Liu, X., Fincher, L., McKearin, D., and Liu, Q. (2005). Dicer-1 and R3D1L catalyze microRNA maturation in Drosophila. Genes \& development 19, 1674-1679.

Jimenez-Mallebrera, C., Torelli, S., Feng, L., Kim, J., Godfrey, C., Clement, E., Mein, R., Abbs, S., Brown, S.C., Campbell, K.P., et al. (2009). A comparative study of alphadystroglycan glycosylation in dystroglycanopathies suggests that the hypoglycosylation of alpha-dystroglycan does not consistently correlate with clinical severity. Brain pathology (Zurich, Switzerland) 19, 596-611.

Johnson, R.P., Kang, S.H., and Kramer, J.M. (2006). C. elegans dystroglycan DGN-1 functions in epithelia and neurons, but not muscle, and independently of dystrophin. Development (Cambridge, England) 133, 1911-1921.

Kheradpour, P., Stark, A., Roy, S., and Kellis, M. (2007). Reliable prediction of regulator targets using 12 Drosophila genomes. Genome research 17, 1919-1931.

Kloosterman, W.P., Wienholds, E., Ketting, R.F., and Plasterk, R.H. (2004). Substrate requirements for let-7 function in the developing zebrafish embryo. Nucleic acids research $32,6284-6291$.

Kojima, S., Chiyomaru, T., Kawakami, K., Yoshino, H., Enokida, H., Nohata, N., Fuse, M., Ichikawa, T., Naya, Y., Nakagawa, M., et al. (2012). Tumour suppressors miR-1 and miR133a target the oncogenic function of purine nucleoside phosphorylase (PNP) in prostate cancer. British journal of cancer 106, 405-413.

Kong, Y., Liang, X., Liu, L., Zhang, D., Wan, C., Gan, Z., and Yuan, L. (2015). High Throughput Sequencing Identifies MicroRNAs Mediating alpha-Synuclein Toxicity by Targeting Neuroactive-Ligand Receptor Interaction Pathway in Early Stage of Drosophila Parkinson's Disease Model. PloS one 10, e0137432.

Koturbash, I., Zemp, F.J., Pogribny, I., and Kovalchuk, O. (2011). Small molecules with big effects: the role of the microRNAome in cancer and carcinogenesis. Mutation research 722, 94-105.

Kreipke, R.E., Kwon, Y.V., Shcherbata, H.R., and Ruohola-Baker, H. (2017). Drosophila melanogaster as a Model of Muscle Degeneration Disorders. Current topics in developmental biology 121, 83-109.

Kucherenko, M.M., Marrone, A.K., Rishko, V.M., Magliarelli Hde, F., and Shcherbata, H.R. (2011). Stress and muscular dystrophy: a genetic screen for dystroglycan and dystrophin 
interactors in Drosophila identifies cellular stress response components. Developmental biology 352, 228-242.

Kucherenko, M.M., Marrone, A.K., Rishko, V.M., Yatsenko, A.S., Klepzig, A., and Shcherbata, H.R. (2010). Paraffin-embedded and frozen sections of Drosophila adult muscles. Journal of visualized experiments : JoVE.

Kucherenko, M.M., and Shcherbata, H.R. (2018a). miRNA targeting and alternative splicing in the stress response - events hosted by membrane-less compartments. Journal of cell science 131 .

Kucherenko, M.M., and Shcherbata, H.R. (2018b). Stress-dependent miR-980 regulation of Rbfox1/A2bp1 promotes ribonucleoprotein granule formation and cell survival. Nature communications 9,312 .

Kudoh, H., Ikeda, H., Kakitani, M., Ueda, A., Hayasaka, M., Tomizuka, K., and Hanaoka, K. (2005). A new model mouse for Duchenne muscular dystrophy produced by $2.4 \mathrm{Mb}$ deletion of dystrophin gene using Cre-loxP recombination system. Biochemical and biophysical research communications 328, 507-516.

Kunkel, L.M., Hejtmancik, J.F., Caskey, C.T., Speer, A., Monaco, A.P., Middlesworth, W., Colletti, C.A., Bertelson, C., Muller, U., Bresnan, M., et al. (1986). Analysis of deletions in DNA from patients with Becker and Duchenne muscular dystrophy. Nature 322, 73-77.

Langevin, S.M., Stone, R.A., Bunker, C.H., Lyons-Weiler, M.A., LaFramboise, W.A., Kelly, L., Seethala, R.R., Grandis, J.R., Sobol, R.W., and Taioli, E. (2011). MicroRNA-137 promoter methylation is associated with poorer overall survival in patients with squamous cell carcinoma of the head and neck. Cancer 117, 1454-1462.

Lee, R.C., Feinbaum, R.L., and Ambros, V. (1993). The C. elegans heterochronic gene lin4 encodes small RNAs with antisense complementarity to lin-14. Cell 75, 843-854.

Lee, Y., Ahn, C., Han, J., Choi, H., Kim, J., Yim, J., Lee, J., Provost, P., Radmark, O., Kim, S., et al. (2003). The nuclear RNase III Drosha initiates microRNA processing. Nature 425, 415-419.

Lee, Y., Kim, M., Han, J., Yeom, K.H., Lee, S., Baek, S.H., and Kim, V.N. (2004). MicroRNA genes are transcribed by RNA polymerase II. The EMBO journal 23, 4051-4060. Leung, A.K., and Sharp, P.A. (2007). microRNAs: a safeguard against turmoil? Cell 130, 581-585.

Leung, A.K., and Sharp, P.A. (2010). MicroRNA functions in stress responses. Molecular cell 40, 205-215.

Lewis, B.P., Burge, C.B., and Bartel, D.P. (2005). Conserved seed pairing, often flanked by adenosines, indicates that thousands of human genes are microRNA targets. Cell 120, 1520 .

Lewis, B.P., Shih, I.H., Jones-Rhoades, M.W., Bartel, D.P., and Burge, C.B. (2003). Prediction of mammalian microRNA targets. Cell 115, 787-798. 
Li, F., Xu, Y., Deng, S., Li, Z., Zou, D., Yi, S., Sui, W., Hao, M., and Qiu, L. (2015). MicroRNA-15a/16-1 cluster located at chromosome $13 q 14$ is down-regulated but displays different expression pattern and prognostic significance in multiple myeloma. Oncotarget 6 , 38270-38282.

Li, X., and Carthew, R.W. (2005). A microRNA mediates EGF receptor signaling and promotes photoreceptor differentiation in the Drosophila eye. Cell 123, 1267-1277.

Li, X., Khanna, A., Li, N., and Wang, E. (2011). Circulatory miR34a as an RNAbased, noninvasive biomarker for brain aging. Aging 3, 985-1002.

Li, X., Li, Y., Zhao, L., Zhang, D., Yao, X., Zhang, H., Wang, Y.C., Wang, X.Y., Xia, H., Yan, J., et al. (2014). Circulating Muscle-specific miRNAs in Duchenne Muscular Dystrophy Patients. Molecular therapy Nucleic acids 3, e177.

Li, Y., Wang, F., Lee, J.A., and Gao, F.B. (2006). MicroRNA-9a ensures the precise specification of sensory organ precursors in Drosophila. Genes \& development 20, 2793 2805 .

Liu, N., Landreh, M., Cao, K., Abe, M., Hendriks, G.J., Kennerdell, J.R., Zhu, Y., Wang, L.S., and Bonini, N.M. (2012). The microRNA miR-34 modulates ageing and neurodegeneration in Drosophila. Nature 482, 519-523.

Ma, J., Shang, S., Wang, J., Zhang, T., Nie, F., Song, X., Heping, Z., Zhu, C., Zhang, R., and Hao, D. (2018). Identification of miR-22-3p, miR-92a-3p, and miR-137 in peripheral blood as biomarker for schizophrenia. Psychiatry research $265,70-76$.

Macrae, I.J., Zhou, K., Li, F., Repic, A., Brooks, A.N., Cande, W.Z., Adams, P.D., and Doudna, J.A. (2006). Structural basis for double-stranded RNA processing by Dicer. Science (New York, NY) 311, 195-198.

Makeyev, E.V., and Maniatis, T. (2008). Multilevel regulation of gene expression by microRNAs. Science (New York, NY) 319, 1789-1790.

Marrone, A.K., Edeleva, E.V., Kucherenko, M.M., Hsiao, N.H., and Shcherbata, H.R. (2012). Dg-Dys-Syn1 signaling in Drosophila regulates the microRNA profile. BMC cell biology 13, 26.

Marrone, A.K., Kucherenko, M.M., Rishko, V.M., and Shcherbata, H.R. (2011a). New dystrophin/dystroglycan interactors control neuron behavior in Drosophila eye. BMC neuroscience 12,93 .

Marrone, A.K., Kucherenko, M.M., Wiek, R., Gopfert, M.C., and Shcherbata, H.R. (2011b). Hyperthermic seizures and aberrant cellular homeostasis in Drosophila dystrophic muscles. Scientific reports $1,47$.

Martinez, N.J., Ow, M.C., Barrasa, M.I., Hammell, M., Sequerra, R., Doucette-Stamm, L., Roth, F.P., Ambros, V.R., and Walhout, A.J. (2008). A C. elegans genome-scale microRNA network contains composite feedback motifs with high flux capacity. Genes \& development $22,2535-2549$. 
Mazaud-Guittot, S., Meugnier, E., Pesenti, S., Wu, X., Vidal, H., Gow, A., and Le Magueresse-Battistoni, B. (2010). Claudin 11 deficiency in mice results in loss of the Sertoli cell epithelial phenotype in the testis. Biology of reproduction 82, 202-213.

McCarthy, J.J., and Esser, K.A. (2007). MicroRNA-1 and microRNA-133a expression are decreased during skeletal muscle hypertrophy. Journal of applied physiology (Bethesda, Md : 1985) 102, 306-313.

Meijer, H.A., Smith, E.M., and Bushell, M. (2014). Regulation of miRNA strand selection: follow the leader? Biochemical Society transactions 42, 1135-1140.

Mendell, J.T., and Olson, E.N. (2012). MicroRNAs in stress signaling and human disease. Cell 148, 1172-1187.

Mercuri, E., and Muntoni, F. (2013). Muscular dystrophies. Lancet (London, England) 381, 845-860.

Miska, E.A., Alvarez-Saavedra, E., Abbott, A.L., Lau, N.C., Hellman, A.B., McGonagle, S.M., Bartel, D.P., Ambros, V.R., and Horvitz, H.R. (2007). Most Caenorhabditis elegans microRNAs are individually not essential for development or viability. PLoS genetics 3, e215.

Mok, K.W., Mruk, D.D., Lee, W.M., and Cheng, C.Y. (2012). Spermatogonial stem cells alone are not sufficient to re-initiate spermatogenesis in the rat testis following adjudininduced infertility. International journal of andrology 35, 86-101.

Morikawa, Y., Heallen, T., Leach, J., Xiao, Y., and Martin, J.F. (2017). Dystrophinglycoprotein complex sequesters Yap to inhibit cardiomyocyte proliferation. Nature 547, 227-231.

Munker, R., and Calin, G.A. (2011). MicroRNA profiling in cancer. Clinical science (London, England : 1979) 121, 141-158.

Muntoni, F., Brockington, M., Blake, D.J., Torelli, S., and Brown, S.C. (2002). Defective glycosylation in muscular dystrophy. Lancet (London, England) 360, 1419-1421.

Ng, S.Y., Soh, B.S., Rodriguez-Muela, N., Hendrickson, D.G., Price, F., Rinn, J.L., and Rubin, L.L. (2015). Genome-wide RNA-Seq of Human Motor Neurons Implicates Selective ER Stress Activation in Spinal Muscular Atrophy. Cell stem cell 17, 569-584.

O'Brien, J., Hayder, H., Zayed, Y., and Peng, C. (2018). Overview of MicroRNA Biogenesis, Mechanisms of Actions, and Circulation. Frontiers in endocrinology 9, 402.

O'Donnell, K.A., Wentzel, E.A., Zeller, K.I., Dang, C.V., and Mendell, J.T. (2005). c-Mycregulated microRNAs modulate E2F1 expression. Nature 435, 839-843.

O'Donnell, P.T., Collier, V.L., Mogami, K., and Bernstein, S.I. (1989). Ultrastructural and molecular analyses of homozygous-viable Drosophila melanogaster muscle mutants indicate there is a complex pattern of myosin heavy-chain isoform distribution. Genes \& development 3, 1233-1246. 
Okada, C., Yamashita, E., Lee, S.J., Shibata, S., Katahira, J., Nakagawa, A., Yoneda, Y., and Tsukihara, T. (2009). A high-resolution structure of the pre-microRNA nuclear export machinery. Science (New York, NY) 326, 1275-1279.

Olde Loohuis, N.F., Kos, A., Martens, G.J., Van Bokhoven, H., Nadif Kasri, N., and Aschrafi, A. (2012). MicroRNA networks direct neuronal development and plasticity. Cellular and molecular life sciences : CMLS 69, 89-102.

Olivieri, F., Spazzafumo, L., Santini, G., Lazzarini, R., Albertini, M.C., Rippo, M.R., Galeazzi, R., Abbatecola, A.M., Marcheselli, F., Monti, D., et al. (2012). Age-related differences in the expression of circulating microRNAs: miR-21 as a new circulating marker of inflammaging. Mechanisms of ageing and development 133, 675-685.

Panchal, T., Chen, X., Alchits, E., Oh, Y., Poon, J., Kouptsova, J., Laski, F.A., and Godt, D. (2017). Specification and spatial arrangement of cells in the germline stem cell niche of the Drosophila ovary depend on the Maf transcription factor Traffic jam. PLoS genetics 13, e1006790.

Pasquinelli, A.E., Reinhart, B.J., Slack, F., Martindale, M.Q., Kuroda, M.I., Maller, B., Hayward, D.C., Ball, E.E., Degnan, B., Muller, P., et al. (2000). Conservation of the sequence and temporal expression of let-7 heterochronic regulatory RNA. Nature 408, 8689.

Perotti, M.E., Cattaneo, F., Pasini, M.E., Verni, F., and Hackstein, J.H. (2001). Male sterile mutant casanova gives clues to mechanisms of sperm-egg interactions in Drosophila melanogaster. Molecular reproduction and development 60, 248-259.

Piccirillo, R., Demontis, F., Perrimon, N., and Goldberg, A.L. (2014). Mechanisms of muscle growth and atrophy in mammals and Drosophila. Developmental dynamics : an official publication of the American Association of Anatomists 243, 201-215.

Porter, J.D., Khanna, S., Kaminski, H.J., Rao, J.S., Merriam, A.P., Richmonds, C.R., Leahy, P., Li, J., Guo, W., and Andrade, F.H. (2002). A chronic inflammatory response dominates the skeletal muscle molecular signature in dystrophin-deficient mdx mice. Human molecular genetics 11, 263-272.

Rouillard, A.D., Gundersen, G.W., Fernandez, N.F., Wang, Z., Monteiro, C.D., McDermott, M.G., and Ma'ayan, A. (2016). The harmonizome: a collection of processed datasets gathered to serve and mine knowledge about genes and proteins. Database : the journal of biological databases and curation 2016.

Ruby, J.G., Stark, A., Johnston, W.K., Kellis, M., Bartel, D.P., and Lai, E.C. (2007). Evolution, biogenesis, expression, and target predictions of a substantially expanded set of Drosophila microRNAs. Genome research 17, 1850-1864.

Saito, K., Ishizuka, A., Siomi, H., and Siomi, M.C. (2005). Processing of pre-microRNAs by the Dicer-1-Loquacious complex in Drosophila cells. PLoS biology 3, e235.

Schneider, M., Khalil, A.A., Poulton, J., Castillejo-Lopez, C., Egger-Adam, D., Wodarz, A., Deng, W.M., and Baumgartner, S. (2006). Perlecan and Dystroglycan act at the basal side of 
the Drosophila follicular epithelium to maintain epithelial organization. Development (Cambridge, England) 133, 3805-3815.

Selbach, M., Schwanhausser, B., Thierfelder, N., Fang, Z., Khanin, R., and Rajewsky, N. (2008). Widespread changes in protein synthesis induced by microRNAs. Nature 455, 5863.

Shah, M.Y., Ferrajoli, A., Sood, A.K., Lopez-Berestein, G., and Calin, G.A. (2016). microRNA Therapeutics in Cancer - An Emerging Concept. EBioMedicine 12, 34-42.

Shcherbata, H.R., Yatsenko, A.S., Patterson, L., Sood, V.D., Nudel, U., Yaffe, D., Baker, D., and Ruohola-Baker, H. (2007). Dissecting muscle and neuronal disorders in a Drosophila model of muscular dystrophy. The EMBO journal 26, 481-493.

Shieh, P.B., Kudryashova, E., and Spencer, M.J. (2011). Limb-girdle muscular dystrophy $2 \mathrm{H}$ and the role of TRIM32. Handbook of clinical neurology 101, 125-133.

Silber, J., Lim, D.A., Petritsch, C., Persson, A.I., Maunakea, A.K., Yu, M., Vandenberg, S.R., Ginzinger, D.G., James, C.D., Costello, J.F., et al. (2008). miR-124 and miR-137 inhibit proliferation of glioblastoma multiforme cells and induce differentiation of brain tumor stem cells. BMC medicine 6, 14 .

Smrt, R.D., Szulwach, K.E., Pfeiffer, R.L., Li, X., Guo, W., Pathania, M., Teng, Z.Q., Luo, Y., Peng, J., Bordey, A., et al. (2010). MicroRNA miR-137 regulates neuronal maturation by targeting ubiquitin ligase mind bomb-1. Stem cells (Dayton, Ohio) 28, 1060-1070.

Sokol, N.S., and Ambros, V. (2005). Mesodermally expressed Drosophila microRNA-1 is regulated by Twist and is required in muscles during larval growth. Genes \& development $19,2343-2354$.

Supek, F., Bosnjak, M., Skunca, N., and Smuc, T. (2011). REVIGO summarizes and visualizes long lists of gene ontology terms. PloS one 6, e21800.

Szklarczyk, D., Franceschini, A., Wyder, S., Forslund, K., Heller, D., Huerta-Cepas, J., Simonovic, M., Roth, A., Santos, A., Tsafou, K.P., et al. (2015). STRING v10: proteinprotein interaction networks, integrated over the tree of life. Nucleic acids research 43, D447-452.

Takeuchi, K., Nakano, Y., Kato, U., Kaneda, M., Aizu, M., Awano, W., Yonemura, S., Kiyonaka, S., Mori, Y., Yamamoto, D., et al. (2009). Changes in temperature preferences and energy homeostasis in dystroglycan mutants. Science (New York, NY) 323, 1740-1743.

Taylor, M.V. (2006). Comparison of Muscle Development in Drosophila and Vertebrates. In Muscle Development in Drosophila (New York, NY: Springer New York), pp. 169-203.

Tinsley, J.M., Blake, D.J., Zuellig, R.A., and Davies, K.E. (1994). Increasing complexity of the dystrophin-associated protein complex. Proceedings of the National Academy of Sciences of the United States of America 91, 8307-8313. 
Tsurudome, K., Tsang, K., Liao, E.H., Ball, R., Penney, J., Yang, J.S., Elazzouzi, F., He, T., Chishti, A., Lnenicka, G., et al. (2010). The Drosophila miR-310 cluster negatively regulates synaptic strength at the neuromuscular junction. Neuron $68,879-893$.

Tufekci, K.U., Oner, M.G., Meuwissen, R.L., and Genc, S. (2014). The role of microRNAs in human diseases. Methods in molecular biology (Clifton, NJ) 1107, 33-50.

Ueyama, M., Akimoto, Y., Ichimiya, T., Ueda, R., Kawakami, H., Aigaki, T., and Nishihara, S. (2010). Increased apoptosis of myoblasts in Drosophila model for the Walker-Warburg syndrome. PloS one 5, e11557.

van der Plas, M.C., Pilgram, G.S., Plomp, J.J., de Jong, A., Fradkin, L.G., and Noordermeer, J.N. (2006). Dystrophin is required for appropriate retrograde control of neurotransmitter release at the Drosophila neuromuscular junction. The Journal of neuroscience : the official journal of the Society for Neuroscience 26, 333-344.

van Reeuwijk, J., Maugenre, S., van den Elzen, C., Verrips, A., Bertini, E., Muntoni, F., Merlini, L., Scheffer, H., Brunner, H.G., Guicheney, P., et al. (2006). The expanding phenotype of POMT1 mutations: from Walker-Warburg syndrome to congenital muscular dystrophy, microcephaly, and mental retardation. Human mutation 27, 453-459.

Vasudevan, S. (2012). Posttranscriptional upregulation by microRNAs. Wiley interdisciplinary reviews RNA 3, 311-330.

Verma, P., Augustine, G.J., Ammar, M.R., Tashiro, A., and Cohen, S.M. (2015). A neuroprotective role for microRNA miR-1000 mediated by limiting glutamate excitotoxicity. Nature neuroscience 18, 379-385.

Waite, A., Brown, S.C., and Blake, D.J. (2012). The dystrophin-glycoprotein complex in brain development and disease. Trends in neurosciences 35, 487-496.

Wang, G., van der Walt, J.M., Mayhew, G., Li, Y.J., Zuchner, S., Scott, W.K., Martin, E.R., and Vance, J.M. (2008). Variation in the miRNA-433 binding site of FGF20 confers risk for Parkinson disease by overexpression of alpha-synuclein. American journal of human genetics $82,283-289$.

Wightman, B., Ha, I., and Ruvkun, G. (1993). Posttranscriptional regulation of the heterochronic gene lin-14 by lin- 4 mediates temporal pattern formation in C. elegans. Cell $75,855-862$.

Willemsen, M.H., Valles, A., Kirkels, L.A., Mastebroek, M., Olde Loohuis, N., Kos, A., Wissink-Lindhout, W.M., de Brouwer, A.P., Nillesen, W.M., Pfundt, R., et al. (2011). Chromosome 1p21.3 microdeletions comprising DPYD and MIR137 are associated with intellectual disability. Journal of medical genetics $48,810-818$.

Wilson, K., Faelan, C., Patterson-Kane, J.C., Rudmann, D.G., Moore, S.A., Frank, D., Charleston, J., Tinsley, J., Young, G.D., and Milici, A.J. (2017). Duchenne and Becker Muscular Dystrophies: A Review of Animal Models, Clinical End Points, and Biomarker Quantification. Toxicologic pathology 45, 961-976. 
Winter, J., Jung, S., Keller, S., Gregory, R.I., and Diederichs, S. (2009). Many roads to maturity: microRNA biogenesis pathways and their regulation. Nature cell biology 11, 228234.

Wu, C.I., Shen, Y., and Tang, T. (2009). Evolution under canalization and the dual roles of microRNAs: a hypothesis. Genome research 19, 734-743.

Wu, L., Fan, J., and Belasco, J.G. (2006). MicroRNAs direct rapid deadenylation of mRNA. Proceedings of the National Academy of Sciences of the United States of America 103, 4034-4039.

Xiao, C., Calado, D.P., Galler, G., Thai, T.H., Patterson, H.C., Wang, J., Rajewsky, N., Bender, T.P., and Rajewsky, K. (2007). MiR-150 controls B cell differentiation by targeting the transcription factor c-Myb. Cell 131, 146-159.

Xie, J., Ameres, S.L., Friedline, R., Hung, J.H., Zhang, Y., Xie, Q., Zhong, L., Su, Q., He, R., Li, M., et al. (2012). Long-term, efficient inhibition of microRNA function in mice using rAAV vectors. Nature methods 9, 403-409.

Xiong, Y., Zhou, Y., and Jarrett, H.W. (2009). Dystrophin glycoprotein complex-associated Gbetagamma subunits activate phosphatidylinositol-3-kinase/Akt signaling in skeletal muscle in a laminin-dependent manner. Journal of cellular physiology 219, 402-414.

Yatsenko, A.S., Gray, E.E., Shcherbata, H.R., Patterson, L.B., Sood, V.D., Kucherenko, M.M., Baker, D., and Ruohola-Baker, H. (2007). A putative Src homology 3 domain binding motif but not the C-terminal dystrophin WW domain binding motif is required for dystroglycan function in cellular polarity in Drosophila. The Journal of biological chemistry 282, 15159-15169.

Yatsenko, A.S., Kucherenko, M.M., Pantoja, M., Fischer, K.A., Madeoy, J., Deng, W.M., Schneider, M., Baumgartner, S., Akey, J., Shcherbata, H.R., et al. (2009). The conserved WW-domain binding sites in Dystroglycan C-terminus are essential but partially redundant for Dystroglycan function. BMC developmental biology 9, 18.

Yatsenko, A.S., Marrone, A.K., and Shcherbata, H.R. (2014). miRNA-based buffering of the cobblestone-lissencephaly-associated extracellular matrix receptor dystroglycan via its alternative 3'-UTR. Nature communications 5, 4906.

Yatsenko, A.S., and Shcherbata, H.R. (2014). Drosophila miR-9a targets the ECM receptor Dystroglycan to canalize myotendinous junction formation. Developmental cell 28, 335348.

Yekta, S., Shih, I.H., and Bartel, D.P. (2004). MicroRNA-directed cleavage of HOXB8 mRNA. Science (New York, NY) 304, 594-596.

Zhang, B., Wang, Q., and Pan, X. (2007). MicroRNAs and their regulatory roles in animals and plants. Journal of cellular physiology 210, 279-289.

Zhang, H., Kolb, F.A., Jaskiewicz, L., Westhof, E., and Filipowicz, W. (2004). Single processing center models for human Dicer and bacterial RNase III. Cell 118, 57-68. 
Zhou, Y.W., Thomason, D.B., Gullberg, D., and Jarrett, H.W. (2006). Binding of laminin alpha1-chain LG4-5 domain to alpha-dystroglycan causes tyrosine phosphorylation of syntrophin to initiate Rac1 signaling. Biochemistry 45, 2042-2052.

Zimmerman, S.G., Peters, N.C., Altaras, A.E., and Berg, C.A. (2013). Optimized RNA ISH, RNA FISH and protein-RNA double labeling (IF/FISH) in Drosophila ovaries. Nature protocols $8,2158-2179$.

Zimmermann, C., Stevant, I., Borel, C., Conne, B., Pitetti, J.L., Calvel, P., Kaessmann, H., Jegou, B., Chalmel, F., and Nef, S. (2015). Research resource: the dynamic transcriptional profile of sertoli cells during the progression of spermatogenesis. Molecular endocrinology (Baltimore, Md) 29, 627-642. 


\section{Supplementary Figures}
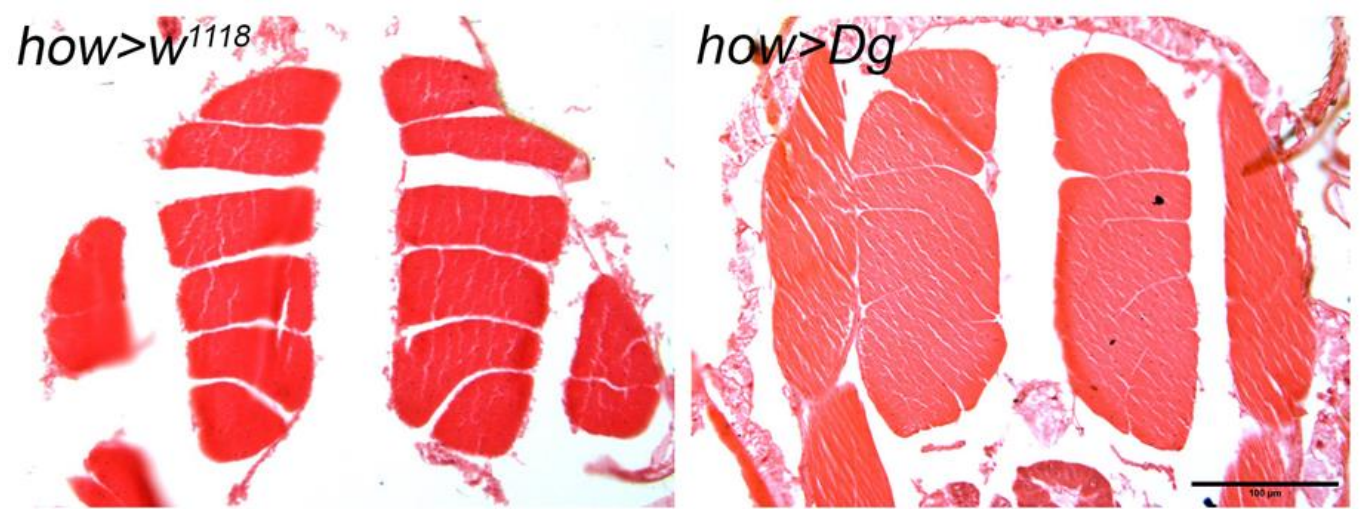

Supplementary Figure 1. Over-expression of Dg in muscle during development results in fused muscle phenotype

Dg over-expression during the developmental stage is semi-lethal and few escapers that are analyzed have fused muscle. No muscle degeneration or the muscle atrophy was observed in these flies. Scale bar $100 \mu \mathrm{m}$. See also Supplementary Table 4.

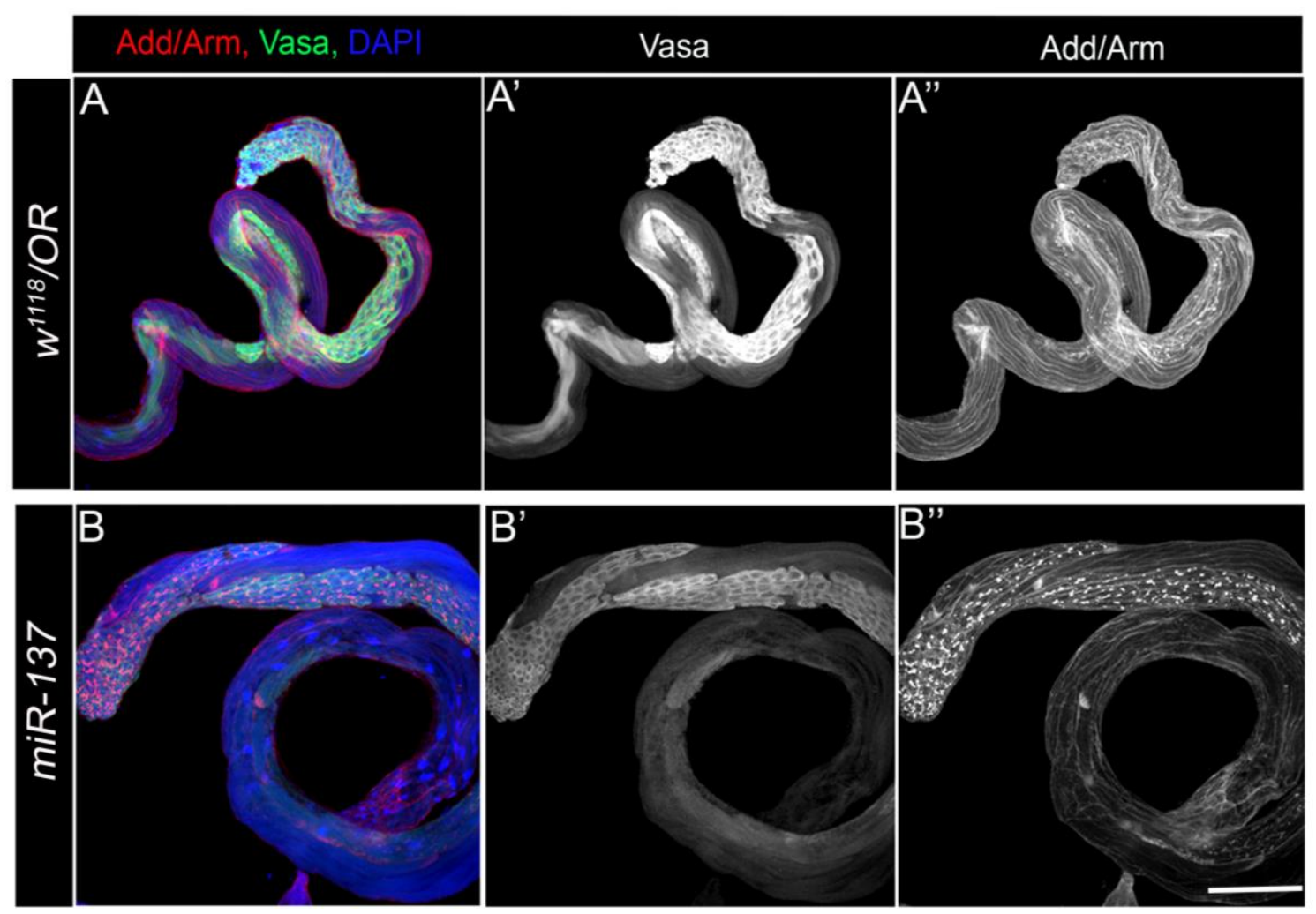

\section{Supplementary Figure 2. MiR-137 mutants have delayed in differentiation}

Antibody staining showing dividing germline in control (A) and in miR-137 mutant testis. Anti-Add staining was more prevalent in miR-137 mutant testis compared to control flies indicating, germline cells are possibly differentiating slower than it has been observed for the control. Scale bar $100 \mu \mathrm{m}$. 


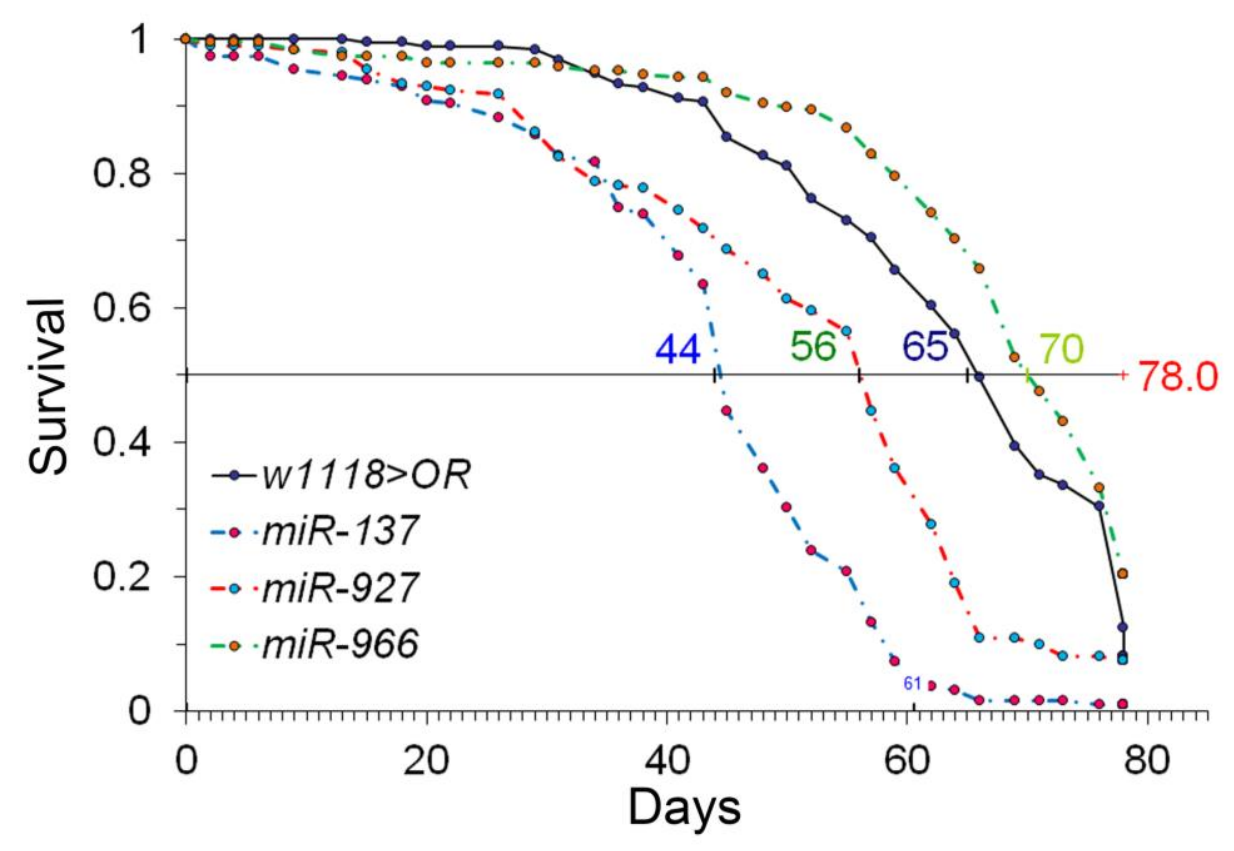

\section{Supplementary Figure 3. Lifespan analysis on miRNA mutants}

Lifespan analysis on candidate miRNA mutants showing reduced lifespan on $m i R-137$ (blue dashed line) and $m i R-927$ (red dashed line) with median survival of 44 and 56 days compared to control genotype $w^{1118}>O R$ (solid black line) with median survival of 65 days. Though, miR-966 (green dashed line) on the other hand showed extended lifespan with median survival of 70 days it is not statistical significant compared to controls. Statistics were analyzed using Log Rank Test, p-value $* * *<0.001)$. 


\section{Supplementary tables}

Supplementary Table 1. The DGC components of Drosophila and its functions

\begin{tabular}{|c|c|c|c|c|c|c|}
\hline $\begin{array}{l}\text { Drosophila } \\
\text { DGC } \\
\text { components }\end{array}$ & Gene Function & $\begin{array}{c}\text { Human } \\
\text { homolog(s) }\end{array}$ & $\begin{array}{c}\text { Disease } \\
\text { association }\end{array}$ & $\ominus^{\circ}$ & ○े & $\partial^{\circ}$ \\
\hline Dg & $\begin{array}{l}\text { Actin cytoskeleton reorganization, axon guidance, } \\
\text { establishment or maintenance of cell polarity, egg } \\
\text { chamber formation, wing vein specification, muscle } \\
\text { cell cellular homeostasis, regulation of glycolytic } \\
\text { process, miRNA involved gene silencing, sarcomere } \\
\text { organization, cytoskeleton anchoring at the plasma } \\
\text { membrane, regulation of synaptic activity }\end{array}$ & DAG1 & $\begin{array}{l}\text { Limb-Girdle muscular dystrophy } \\
\text { Congenital muscular dystrophy } \\
\text { Pediatric autoimmune diseases }\end{array}$ & $24 \%$ & $35 \%$ & $28 \%$ \\
\hline Dys & $\begin{array}{l}\text { Establishment of cell polarity, wing vein } \\
\text { morphogenesis, wing vein specification, muscle cell } \\
\text { cellular homeostasis, muscle organ development, } \\
\text { neuromuscular synaptic transmission, regulation of } \\
\text { neurotransmitter secretion, regulation of short-term } \\
\text { neuronal synaptic plasticity }\end{array}$ & $\begin{array}{l}\text { DMD } \\
\text { UTRN }\end{array}$ & $\begin{array}{l}\text { Dilated cardiomyopathy } \\
\text { Duchenne muscular dystrophy } \\
\text { Becker muscular dystrophy }\end{array}$ & $\begin{array}{l}29 \% \\
29 \%\end{array}$ & $\begin{array}{l}47 \% \\
47 \%\end{array}$ & $\begin{array}{l}20 \% \\
17 \%\end{array}$ \\
\hline Syn1 & Locomotion, regulation of synaptic growth at NMJ & $\begin{array}{l}\text { SNTB1 } \\
\text { SNTB2 }\end{array}$ & Myopia, Periodontitis & $\begin{array}{l}39 \% \\
37 \%\end{array}$ & $\begin{array}{l}55 \% \\
52 \%\end{array}$ & $\begin{array}{l}18 \% \\
20 \%\end{array}$ \\
\hline
\end{tabular}




\begin{tabular}{|c|c|c|c|c|c|c|}
\hline Syn2 & $\begin{array}{l}\text { locomotion, eye development, rhabdomere } \\
\text { development, regulation of synaptic growth at NMJ }\end{array}$ & SNTG1 & $\begin{array}{l}\text { Chronic obstructive pulmonary } \\
\text { disease }\end{array}$ & $40 \%$ & $59 \%$ & $2 \%$ \\
\hline Nos & $\begin{array}{l}\text { Nervous system development, regulation of heart } \\
\text { rate, imaginal disc development, synapse assembly, } \\
\text { response to hormone/lipopolysaccharide, regulation } \\
\text { of organ Growth, negative regulation of cell } \\
\text { proliferation and DNA replication }\end{array}$ & NOS1 & $\begin{array}{l}\text { Colorectal cancer } \\
\text { Hypertension } \\
\text { Psoriasis } \\
\text { Alzheimer }\end{array}$ & $44 \%$ & $59 \%$ & $12 \%$ \\
\hline $\operatorname{Scg} \alpha$ & - & SGCE & Myoclonic dystonia & $25 \%$ & $45 \%$ & $16 \%$ \\
\hline $\operatorname{Scg} \beta$ & Muscle organ development & SGCB & Limb-Girdle muscular dystrophy & $27 \%$ & $45 \%$ & $27 \%$ \\
\hline $\operatorname{Scg} \delta$ & $\begin{array}{l}\text { Heart contraction, mesoderm development, } \\
\text { sarcomere organization, cytoskeletal anchoring to } \\
\text { the plasma membrane }\end{array}$ & SGCD & $\begin{array}{l}\text { Limb-Girdle muscular dystrophy } \\
\text { Dilated cardiomyopathy }\end{array}$ & $39 \%$ & $60 \%$ & $3 \%$ \\
\hline Cora & $\begin{array}{l}\text { adult somatic muscle development, dorsal closure, } \\
\text { cell adhesion in heart morphogenesis, embryonic } \\
\text { development, establishment of the blood-brain } \\
\text { barrier, wing hair orientation, regulation of tracheal } \\
\text { tube size, open tracheal system, cell polarity, cell- } \\
\text { cell junction, septate junction assembly }\end{array}$ & $\begin{array}{l}\text { EPB41L3 } \\
\text { EPB41L1 } \\
\text { EPB41L2 }\end{array}$ & $\begin{array}{l}\text { Amyotrophic lateral sclerosis } \\
\text { Colorectal cancer } \\
\text { Mental retardation }\end{array}$ & $\begin{array}{l}25 \% \\
27 \% \\
36 \%\end{array}$ & $\begin{array}{l}35 \% \\
41 \% \\
50 \%\end{array}$ & $\begin{array}{l}43 \% \\
29 \% \\
22 \%\end{array}$ \\
\hline
\end{tabular}




\begin{tabular}{|c|c|c|c|c|c|c|}
\hline Nrx IV & $\begin{array}{l}\text { Axon ensheathment, cell adhesion in heart } \\
\text { morphogenesis, dorsal closure, the establishment of } \\
\text { the glial blood-brain barrier, nerve maturation, } \\
\text { presynaptic membrane assembly, septate junction } \\
\text { assembly, regulation of tube size, an open tracheal } \\
\text { system, synaptic vesicle docking and targeting, } \\
\text { establishment of membrane cell polarity }\end{array}$ & $\begin{array}{l}\text { CNNAP2 } \\
\text { CNTNAP5 }\end{array}$ & $\begin{array}{l}\text { Alzheimer } \\
\text { Bipolar disorder } \\
\text { Schizophrenia } \\
\text { Autism } \\
\text { Cortical dysplasia-Focal epilepsy } \\
\text { syndrome } \\
\text { Rohn's disease }\end{array}$ & $\begin{array}{l}33 \% \\
32 \%\end{array}$ & $\begin{array}{l}51 \% \\
51 \%\end{array}$ & $\begin{array}{l}10 \% \\
9 \%\end{array}$ \\
\hline Yki & $\begin{array}{l}\text { Border cell migration, cell proliferation, cell fate } \\
\text { specification, Hippo-signaling, negative regulation } \\
\text { of the apoptotic process, positive regulation of glial } \\
\text { cell proliferation, growth, histone H3-K4 } \\
\text { methylation, H3-K4 trimethylation, imaginal disc } \\
\text { growth, stem cell proliferation }\end{array}$ & YAP1 & $\begin{array}{l}\text { Coloboma, ocular, with or } \\
\text { without hearing impairment } \\
\text { cleft lip/palate, and/or mental } \\
\text { retardation } \\
\text { MRI atrophy measures, } \\
\text { Polycystic ovary syndrome } \\
\text { Pubertal anthropometrics }\end{array}$ & $31 \%$ & $45 \%$ & $34 \%$ \\
\hline $\operatorname{Lan} A$ & $\begin{array}{l}\text { Axon guidance, cell adhesion, dorsal trunk growth, } \\
\text { an open tracheal system, heart development, } \\
\text { mesoderm development, regulation of cell adhesion, } \\
\text { cell migration and embryonic development, negative } \\
\text { regulation of synaptic growth at NMJ }\end{array}$ & LAMA5 & $\begin{array}{l}\text { Colorectal cancer } \\
\text { Amyotrophic lateral sclerosis } \\
\text { Cardiomyopathy } \\
\text { Congenital muscular dystrophy }\end{array}$ & $32 \%$ & $47 \%$ & $15 \%$ \\
\hline
\end{tabular}




\begin{tabular}{|c|c|c|c|c|c|c|}
\hline LanB2 & $\begin{array}{l}\text { Basement membrane assembly, cell adhesion, } \\
\text { endodermal digestive tract morphogenesis, } \\
\text { extracellular matrix assembly, salivary gland } \\
\text { morphogenesis }\end{array}$ & LAMC1 & $\begin{array}{l}\text { Colorectal cancer } \\
\text { Cortical malformations } \\
\text { Coronary heart disease } \\
\text { Systemic lupus erythematosus } \\
\text { Epidermolysis bullosa }\end{array}$ & $42 \%$ & $59 \%$ & $6 \%$ \\
\hline Mei-P26 & $\begin{array}{l}\text { Gamete generation, germ cell development, meiotic } \\
\text { cell cycle, spermatogenesis }\end{array}$ & $\begin{array}{l}\text { TRIM56 } \\
\text { TRIM2 }\end{array}$ & & $\begin{array}{l}21 \% \\
21 \%\end{array}$ & $\begin{array}{l}34 \% \\
35 \%\end{array}$ & $\begin{array}{l}30 \% \\
27 \%\end{array}$ \\
\hline Arm & $\begin{array}{l}\text { Cell adhesion, wing disc and cell morphogenesis, } \\
\text { dorsal closure, heart formation and development, } \\
\text { long-term memory, oogenesis, delamination, } \\
\text { photoreceptor cell differentiation, segment polarity, } \\
\text { somatic stem cell population maintenance, } \\
\text { neuroblast development, nervous system } \\
\text { development, cuticle pattern formation }\end{array}$ & $\begin{array}{l}\text { CTNNB1 } \\
\text { JUP }\end{array}$ & $\begin{array}{l}\text { Hepatocellular carcinoma, } \\
\text { Ovarian cancer } \\
\text { Mental retardation } \\
\text { Colorectal cancer } \\
\text { Exudative vitreoretinopathy } \\
\text { Medulloblastoma }\end{array}$ & $67 \%$ & $77 \%$ & $9 \%$ \\
\hline Hts & $\begin{array}{l}\text { Actin filament organization, adult somatic muscle } \\
\text { development, axon guidance, centrosome cycle, } \\
\text { cystoblast cycle, oogenesis, female germline ring } \\
\text { cancal formation, meiotic spindle organization, } \\
\text { photoreceptor axon guidance, sarcomere } \\
\text { organization, testicular fusome organization }\end{array}$ & ADD1 & $\begin{array}{l}\text { Cerebral palsy, } \\
\text { spastic quadriplegia } \\
\text { Hypertension }\end{array}$ & $\begin{array}{l}33 \% \\
63 \%\end{array}$ & $\begin{array}{l}49 \% \\
76 \%\end{array}$ & $\begin{array}{l}21 \% \\
4 \%\end{array}$ \\
\hline
\end{tabular}


Supplementary Table 2. Relative transcript levels of $D g$, Dys, and Syn1 due to miRNA loss

\begin{tabular}{|c|c|c|c|c|c|}
\hline Genotype & $\begin{array}{c}\mathrm{C}_{\mathrm{T}} \operatorname{Rpl32} \\
\mathrm{AVE} \pm \mathrm{STDEV}^{\mathrm{a}, \mathrm{b}} \\
\end{array}$ & $\mathrm{C}_{\mathrm{T}} \mathrm{Dg}$ & $\Delta \mathbf{C}_{\mathrm{T}}$ & $\Delta \Delta C_{T}$ & 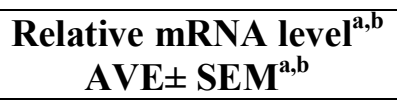 \\
\hline Canton-S/OR & $\begin{array}{l}21.247 \\
\pm 0.030\end{array}$ & $\begin{array}{c}25.482 \\
\pm 0.079\end{array}$ & 4.196 & 0 & $\begin{array}{l}1.000 \\
\pm 0.07\end{array}$ \\
\hline Canton-S/OR & $\begin{array}{l}16.856 \\
\pm 0.064\end{array}$ & $\begin{array}{l}21.640 \\
\pm 0.206\end{array}$ & 4.784 & 0 & $\begin{array}{c}1.000 \\
\pm 0.154\end{array}$ \\
\hline$m i R-137^{b}$ & $\begin{array}{l}25.002 \\
\pm 0.027\end{array}$ & $\begin{array}{l}27.922 \\
\pm 0.112\end{array}$ & 2.921 & -1.863 & $\begin{array}{c}3.638 \\
\pm 0.253\end{array}$ \\
\hline$m i R-252^{a}$ & $\begin{array}{l}20.905 \\
\pm 0.083\end{array}$ & $\begin{array}{l}23.475 \\
\pm 0.293\end{array}$ & 2.570 & -1.628 & $\begin{array}{c}3.09 \\
\pm 0.099\end{array}$ \\
\hline$m i R-310 s^{b}$ & $\begin{array}{r}15.820 \\
\pm 0.194 \\
\end{array}$ & $\begin{array}{l}20.371 \\
\pm 0.084\end{array}$ & 4.555 & -0.229 & $\begin{aligned} & 1.172 \\
\pm & 0.141\end{aligned}$ \\
\hline$m i R-927^{b}$ & $\begin{array}{l}23.734 \\
\pm 0.135\end{array}$ & $\begin{array}{l}29.578 \\
\pm 0.135\end{array}$ & 5.843 & 1.059 & $\begin{array}{c}0.479 \\
\pm 0.093\end{array}$ \\
\hline$m i R-956^{a}$ & $\begin{array}{r}18.634 \\
\pm 0.061\end{array}$ & $\begin{array}{c}24.515 \\
\pm 0.080\end{array}$ & 5.880 & 1.682 & $\begin{array}{c}0.311 \\
\pm 0.099\end{array}$ \\
\hline$m i R-959 C^{a}$ & $\begin{array}{l}23.242 \\
\pm 0.230\end{array}$ & $\begin{array}{l}25.536 \\
\pm 0.077\end{array}$ & 2.294 & -1.904 & $\begin{array}{c}3.743 \\
\pm 0.111\end{array}$ \\
\hline$m i R-966^{b}$ & $\begin{array}{r}18.277 \\
\pm 0.310\end{array}$ & $\begin{array}{l}21.176 \\
\pm 0.163\end{array}$ & 3.536 & -1.248 & $\begin{array}{c}2.375 \\
\pm 0.531 \\
\end{array}$ \\
\hline$m i R-975 C^{b}$ & $\begin{array}{l}23.889 \\
\pm 0.135\end{array}$ & $\begin{array}{l}28.754 \\
\pm 0.126\end{array}$ & 4.865 & 0.089 & $\begin{array}{c}0.945 \\
\pm 0.091\end{array}$ \\
\hline$m i R-1000^{b}$ & $\begin{array}{l}25.455 \\
\pm 0.158\end{array}$ & $\begin{array}{l}28.065 \\
\pm 0.079\end{array}$ & 2.610 & -2.174 & $\begin{array}{c}4.513 \\
\pm 0.103\end{array}$ \\
\hline$m i R-1011 /+^{b}$ & $\begin{array}{r}11.466 \\
\pm 0.653 \\
\end{array}$ & $\begin{array}{r}14.912 \\
\pm 1.185 \\
\end{array}$ & 5.437 & 0.656 & $\begin{array}{c}0.636 \\
\pm 0.129 \\
\end{array}$ \\
\hline Genotype & $\begin{array}{c}\mathrm{C}_{\mathrm{T}} \operatorname{Rpl32} \\
\mathrm{AVE} \pm \mathrm{STDEV} \mathbf{S}^{\mathrm{a}, \mathrm{b}}\end{array}$ & $\mathrm{C}_{\mathrm{T}} \operatorname{Dys}$ & $\Delta \mathbf{C}_{\mathbf{T}}$ & $\Delta \Delta C_{T}$ & $\begin{array}{c}\text { Relative mRNA level }^{\mathrm{a}, \mathrm{b}} \\
\text { AVE } \pm \mathrm{SEM}^{\mathrm{a}, \mathrm{b}}\end{array}$ \\
\hline Canton-S/OR & $\begin{array}{r}16.167 \\
\pm 0.040\end{array}$ & $\begin{array}{l}21.891 \\
\pm 0.040\end{array}$ & 5.438 & 0 & $\begin{array}{l}1.000 \\
\pm 0.011\end{array}$ \\
\hline Canton-S/OR & $\begin{array}{l}21.268 \\
\pm 0.030\end{array}$ & $\begin{array}{l}25.482 \\
\pm 0.077\end{array}$ & 4.214 & 0 & $\begin{aligned} & 1.00 \\
\pm & 0.070\end{aligned}$ \\
\hline$m i R-137^{a}$ & $\begin{array}{c}17.931 \\
\pm 0.0126\end{array}$ & $\begin{array}{l}25.482 \\
\pm 0.077\end{array}$ & 3.836 & -1.641 & $\begin{array}{c}3.118 \\
\pm 0.034\end{array}$ \\
\hline$m i R-137^{b}$ & $\begin{array}{l}24.229 \\
\pm 0.386\end{array}$ & $\begin{array}{l}27.501 \\
\pm 0.149\end{array}$ & 3.216 & -0.998 & $\begin{array}{c}1.998 \\
\pm 0.120\end{array}$ \\
\hline$m i R-252^{a}$ & $\begin{array}{r}18.571 \\
\pm 0.105\end{array}$ & $\begin{array}{l}23.524 \\
\pm 0.166\end{array}$ & 5.094 & -0.383 & $\begin{array}{c}1.304 \\
\pm 0.108 \\
\end{array}$ \\
\hline$m i R-252^{b}$ & $\begin{array}{l}21.234 \\
\pm 0.029\end{array}$ & $\begin{array}{c}24.265 \\
\pm 0.059\end{array}$ & 3.059 & -1.155 & $\begin{array}{c}2.227 \\
\pm 0.108\end{array}$ \\
\hline$m i R-310 s^{a}$ & $\begin{array}{r}19.805 \\
\pm 0.205\end{array}$ & $\begin{array}{c}23.794 \\
\pm 0.106\end{array}$ & 3.917 & -1.559 & $\begin{array}{c}2.947 \\
\pm 0.04\end{array}$ \\
\hline $\operatorname{miR}-927^{a}$ & $\begin{array}{c}17.546 \\
\pm 0.049\end{array}$ & $\begin{array}{c}23.221 \\
\pm 0.342\end{array}$ & 5.532 & 0.055 & $\begin{array}{c}0.963 \\
\pm 0.266\end{array}$ \\
\hline$m i R-956^{b}$ & $\begin{array}{r}24.003 \\
\pm 0.031\end{array}$ & $\begin{array}{c}28.451 \\
\pm 0.856\end{array}$ & 4.448 & 0.233 & $\begin{array}{c}0.851 \\
\pm 0.227 \\
\end{array}$ \\
\hline$m i R-959 C^{b}$ & $\begin{array}{c}18.340 \\
\pm 0.110\end{array}$ & $\begin{array}{c}23.209 \\
\pm 0.119\end{array}$ & 4.926 & 0.712 & $\begin{array}{c}0.645 \\
\pm 0.046\end{array}$ \\
\hline
\end{tabular}




\begin{tabular}{|c|c|c|c|c|c|}
\hline$m i R-966^{a}$ & $\begin{array}{r}20.720 \\
\pm 0.159 \\
\end{array}$ & $\begin{array}{r}23.703 \\
\pm 0.327 \\
\end{array}$ & 3.143 & -2.334 & $\begin{array}{c}5.042 \\
\pm 0.174 \\
\end{array}$ \\
\hline$m i R-975 C^{b}$ & $\begin{array}{r}19.706 \\
\pm 0.073\end{array}$ & $\begin{array}{l}23.787 \\
\pm 0.079\end{array}$ & 4.081 & -0.133 & $\begin{aligned} & 1.097 \\
\pm & 0.079\end{aligned}$ \\
\hline$m i R-1000^{b}$ & $\begin{array}{r}18.099 \\
\pm 0.121 \\
\end{array}$ & $\begin{array}{r}22.459 \\
\pm 0.225 \\
\end{array}$ & 4.359 & 0.149 & $\begin{array}{c}0.905 \\
\pm 0.159 \\
\end{array}$ \\
\hline$m i R-1011 /+^{a}$ & $\begin{array}{r}16.493 \\
\pm 0.244 \\
\end{array}$ & $\begin{array}{l}20.065 \\
\pm 0.040 \\
\end{array}$ & 3.479 & -1.999 & $\begin{array}{r}3.996 \\
\pm 0.213 \\
\end{array}$ \\
\hline Genotype & $\begin{array}{c}\mathrm{C}_{\mathrm{T}} \boldsymbol{R p l 3 2} \\
\mathrm{AVE} \pm \mathrm{STDEV}^{\mathrm{a}, \mathrm{b}}\end{array}$ & $\mathrm{C}_{\mathrm{T}}$ Syn1 & $\Delta \mathbf{C}_{\mathrm{T}}$ & $\Delta \Delta \mathbf{C}_{\mathrm{T}}$ & $\begin{array}{c}\text { Relative mRNA level }{ }^{\mathrm{a}, \mathrm{b}} \\
\text { AVE } \pm \text { SEM }^{\mathrm{a}, \mathrm{b}}\end{array}$ \\
\hline Canton-S/OR & $\begin{array}{l}25.442 \\
\pm 0.119\end{array}$ & $\begin{array}{r}26.557 \\
\pm 0.097 \\
\end{array}$ & 1.049 & 0 & $\begin{array}{l}1.000 \\
\pm 0.177\end{array}$ \\
\hline Canton-S/OR & $\begin{array}{c}16.183 \\
\pm 0.266 \\
\end{array}$ & $\begin{array}{r}21.119 \\
\pm 0.175 \\
\end{array}$ & 4.939 & 0 & $\begin{array}{r}1.000 \\
\pm 0.138 \\
\end{array}$ \\
\hline $\operatorname{miR}-137^{a}$ & $\begin{array}{l}27.914 \\
\pm 0.286 \\
\end{array}$ & $\begin{array}{r}29.786 \\
\pm 0.157 \\
\end{array}$ & 1.121 & 0.163 & $\begin{array}{c}0.893 \\
\pm 0.719 \\
\end{array}$ \\
\hline$m i R-137^{b}$ & $\begin{array}{r}17.646 \\
\pm 0.191 \\
\end{array}$ & $\begin{array}{c}24.171 \\
\pm 0.098\end{array}$ & 6.525 & 1.586 & $\begin{array}{c}0.333 \\
\pm 0.065 \\
\end{array}$ \\
\hline$m i R-252^{a}$ & $\begin{array}{l}20.823 \\
\pm 0.193\end{array}$ & $\begin{array}{l}23.031 \\
\pm 0.099\end{array}$ & 2.208 & 1.158 & $\begin{array}{c}0.448 \\
\pm 0.187\end{array}$ \\
\hline$m i R-310 s^{b}$ & $\begin{array}{r}19.368 \\
\pm 0.137\end{array}$ & $\begin{array}{l}23.831 \\
\pm 0.287\end{array}$ & 3.796 & -1.14 & $\begin{array}{c}2.209 \\
\pm 0.070\end{array}$ \\
\hline$m i R-927^{a}$ & $\begin{array}{l}26.992 \\
\pm 0.066\end{array}$ & $\begin{array}{l}28.458 \\
\pm 0.174\end{array}$ & 1.918 & 0.868 & $\begin{array}{c}0.548 \\
\pm 0.528\end{array}$ \\
\hline$m i R-927^{b}$ & $\begin{array}{r}17.419 \\
\pm 0.291\end{array}$ & $\begin{array}{r}24.067 \\
\pm 0.021\end{array}$ & 6.647 & 1.707 & $\begin{array}{c}0.306 \\
\pm 0.128\end{array}$ \\
\hline$m i R-956^{a}$ & $\begin{array}{r}24.724 \\
\pm 0.182\end{array}$ & $\begin{array}{l}28.898 \\
\pm 0.310\end{array}$ & 3.931 & 2.882 & $\begin{array}{c}0.136 \\
\pm 0.095\end{array}$ \\
\hline$m i R-959 C^{a}$ & $\begin{array}{l}21.889 \\
\pm 0.177\end{array}$ & $\begin{array}{l}27.581 \\
\pm 0.538\end{array}$ & 6.025 & 4.975 & $\begin{array}{c}0.032 \\
\pm 0.078\end{array}$ \\
\hline$m i R-966^{b}$ & $\begin{array}{l}20.593 \\
\pm 0.099\end{array}$ & $\begin{array}{r}24.260 \\
\pm 0.075\end{array}$ & 3.641 & -1.298 & $\begin{array}{c}2.460 \\
\pm 0.123\end{array}$ \\
\hline$m i R-975 C^{a}$ & $\begin{array}{l}26.054 \\
\pm 0.257 \\
\end{array}$ & $\begin{array}{r}28.173 \\
\pm 0.918 \\
\end{array}$ & 2.119 & 1.069 & $\begin{array}{c}0.477 \\
\pm 0.174 \\
\end{array}$ \\
\hline$m i R-1000^{a}$ & $\begin{array}{l}23.397 \\
\pm 0.464 \\
\end{array}$ & $\begin{array}{l}27.631 \\
\pm 0.485\end{array}$ & 4.197 & 3.147 & $\begin{array}{c}0.113 \\
\pm 0.116 \\
\end{array}$ \\
\hline$m i R-1011 /+^{b}$ & $\begin{array}{r}15.755 \\
\pm 0.161 \\
\end{array}$ & $\begin{array}{c}21.192 \\
\pm 0.066 \\
\end{array}$ & 5.428 & 0.488 & $\begin{array}{c}0.713 \\
\pm 0.178 \\
\end{array}$ \\
\hline
\end{tabular}

${ }^{\mathrm{a}}$ and ${ }^{\mathrm{b}}$ represent separate $\mathrm{qPCR}$ runs. The relative mRNA levels were calculated using the formula $2^{-\triangle \Delta C T}$. An average of two runs when applied was plotted in the graph. Average. Standard deviation $(\mathrm{AVE} \pm \mathrm{STDEV})$ values are based on three replicates. $\mathrm{P}$ values are calculated using two-tailed non-paired Student's t-test for significance testing

Supplementary Table 3. Percentage of muscle degeneration in miRNA mutants

\begin{tabular}{|c|c|c|c|c|c|c|}
\hline \multirow{3}{*}{$\begin{array}{l}\text { Genotype } \\
\text { Canton-S/OR }{ }^{\mathrm{D} 7}\end{array}$} & \multicolumn{3}{|c|}{$\%$ of muscle degeneration } & \multirow{2}{*}{$\mathbf{n}$} & \multirow{2}{*}{\multicolumn{2}{|c|}{$\begin{array}{c}\mathrm{X}^{2} \text { test with } \\
\text { Yate's correction }\end{array}$}} \\
\hline & Mild & Moderate & Strong & & & \\
\hline & 3.870 & - & - & 155 & - & - \\
\hline Canton-S/OR & 2.817 & 3.521 & 1.408 & 142 & - & 9.655 \\
\hline Canton-S/ORSS & 4.167 & 3.333 & 2.500 & 120 & - & 11.534 \\
\hline Canton-S/ORPS & 4.167 & - & 0.684 & 144 & - & 5.165 \\
\hline
\end{tabular}




\begin{tabular}{|c|c|c|c|c|c|c|}
\hline $\begin{array}{l}\text { Canton- } \\
\text { S/OR }\end{array}$ & 4.109 & 1.369 & 0.684 & 146 & - & 7.356 \\
\hline $\operatorname{miR}-137^{\mathrm{D} 7}$ & 3.597 & - & - & 139 & 0.114 & - \\
\hline miR-137 ${ }^{\text {TS }}$ & 10.126 & 6.329 & 3.797 & 79 & $8.065^{*}$ & $17.191^{* * *}$ \\
\hline $\mathrm{miR}-137^{\mathrm{SS}}$ & 18.103 & 2.586 & - & 116 & $14.743^{\star *}$ & $18.799^{* * *}$ \\
\hline miR-137 & 4.762 & 5.952 & 11.905 & 168 & $21.963^{\star \star \star}$ & $9.429^{*}$ \\
\hline miR-137 D30 & 9.957 & 4.762 & 3.030 & 231 & $11.484^{* *}$ & $20.256^{* * *}$ \\
\hline$m i R-252^{\mathrm{D} 7}$ & 7.395 & 0.965 & - & 311 & 5.2 & - \\
\hline$m i R-252^{\top S}$ & 6.944 & 1.389 & - & 144 & 5.847 & 0.399 \\
\hline$m i R-252^{S S}$ & 12.307 & 0.769 & 3.846 & 130 & $8.053^{*}$ & 0.399 \\
\hline$m i R-252^{\text {PS }}$ & 7.692 & 1.183 & 0.592 & 169 & 2.621 & $9.746^{*}$ \\
\hline$m i R-252^{\mathrm{D} 30}$ & 5.556 & 3.472 & 3.472 & 144 & 5.913 & $21.387^{*}$ \\
\hline$m i R-310 s^{D 7}$ & 12.903 & - & - & 124 & 7.671 & - \\
\hline$m i R-310 s^{\top S}$ & 4.00 & 2.667 & 1.667 & 300 & $35.523^{* \star *}$ & $14.378^{* *}$ \\
\hline$m i R-310 s^{S S}$ & & & & & $\mathrm{~N} / \mathrm{A}$ & $\mathrm{N} / \mathrm{A}$ \\
\hline$m i R-310 s^{P S}$ & 14.110 & 4.294 & 1.226 & 163 & $12.933^{* \star}$ & $11.885^{* *}$ \\
\hline$m i R-310 s^{\mathrm{D} 30}$ & 5.833 & 0.833 & - & 120 & 3.778 & 5.556 \\
\hline$m i R-927^{D 7}$ & 3.333 & - & - & 120 & 0.179 & - \\
\hline$m i R-927^{T S}$ & 3.086 & 3.704 & 0.617 & 162 & 0.507 & 7.196 \\
\hline$m i R-927^{s s}$ & 7.547 & 0.943 & 2.830 & 106 & 3.079 & $9.636^{*}$ \\
\hline miR-927PS & 18.717 & 1.069 & 2.139 & 187 & $18.518^{* * *}$ & $16.144^{* *}$ \\
\hline$m i R-927^{030}$ & 7.291 & 5.208 & 2.083 & 96 & 3.282 & $12.624^{* *}$ \\
\hline$m i R-956^{\mathrm{D} 7}$ & 4.167 & - & - & 144 & 0.167 & - \\
\hline$m i R-956^{\top \mathrm{TS}}$ & 1.389 & 4.167 & - & 72 & 0.101 & 6.984 \\
\hline$m i R-956^{\text {sS }}$ & 9.090 & 1.515 & 1.515 & 132 & 3.876 & $9.262^{*}$ \\
\hline$m i R-956^{\mathrm{PS}}$ & 3.125 & 3.906 & 2.343 & 128 & 3.639 & $7.791^{*}$ \\
\hline$m i R-956^{\mathrm{D} 30}$ & 1.667 & - & 0.833 & 120 & $13.231^{* *}$ & 3.985 \\
\hline$m i R-959 C^{\mathrm{D} 7}$ & 13.559 & 0.847 & 0.847 & 236 & $14.963^{* *}$ & - \\
\hline$m i R-959 C^{\top S}$ & 5.833 & - & - & 120 & 7.35 & $10.983^{*}$ \\
\hline miR-959C ${ }^{\mathrm{ss}}$ & 1.923 & 4.807 & - & 104 & 4.615 & $19.802^{* \star \star}$ \\
\hline$m i R-959 C^{\mathrm{PS}}$ & 12.962 & 4.321 & 12.963 & 162 & $31.226^{* \star *}$ & $32.874^{* * *}$ \\
\hline$m i R-959 C^{\mathrm{D} 30}$ & 2.586 & - & - & 116 & $9.319^{*}$ & $16.903^{* * *}$ \\
\hline$m i R-966^{\mathrm{D} 7}$ & 2.778 & 1.389 & - & 144 & 2.96 & - \\
\hline$m i R-966^{\top S}$ & 10.949 & 2.189 & - & 137 & $9.358^{*}$ & $8.262^{*}$ \\
\hline$m i R-966^{\mathrm{SS}}$ & 3.677 & 2.206 & 5.147 & 136 & 1.843 & $12.425^{\text {** }}$ \\
\hline$m i R-966^{\mathrm{PS}}$ & 15.2 & 2.7 & - & 224 & $14.632^{* *}$ & $69.911^{* * *}$ \\
\hline$m i R-966^{\mathrm{D} 30}$ & 3.333 & - & - & 120 & $8.415^{*}$ & 4.815 \\
\hline$m i R-975 C^{\mathrm{D} 7}$ & - & 1.438 & 6.475 & 139 & 3.763 & - \\
\hline$m i R-975 C^{\top S}$ & 5.699 & 2.591 & 11.917 & 193 & $14.018^{* *}$ & $16.358^{* * *}$ \\
\hline$m i R-975 C^{S S}$ & 2.500 & 5.833 & 8.333 & 120 & 3.122 & $9.941^{*}$ \\
\hline$m i R-975 C^{\mathrm{PS}}$ & - & - & 24.432 & 176 & $29.68^{\star \star \star}$ & $64.277^{\text {*** }}$ \\
\hline$m i R-975 C^{\mathrm{D} 30}$ & 3.097 & 1.327 & 2.212 & 226 & $10.719^{*}$ & 7.138 \\
\hline$m i R-1000^{\mathrm{D} 7}$ & 10.823 & 5.628 & 1.299 & 231 & $19.072^{* * *}$ & - \\
\hline$m i R-1000^{\top S}$ & 3.125 & - & 6.250 & 96 & 7.409 & $84.873^{* * *}$ \\
\hline $\operatorname{miR}-1000^{\mathrm{sS}}$ & 8.3 & 0.9 & - & 108 & 6.456 & $64.826^{* * *}$ \\
\hline$m i R-1000^{P S}$ & \multicolumn{4}{|c|}{ lethal } & $\mathrm{N} / \mathrm{A}$ & $\mathrm{N} / \mathrm{A}$ \\
\hline$m i R-1000^{\mathrm{D} 30}$ & \multicolumn{4}{|c|}{ lethal } & $\mathrm{N} / \mathrm{A}$ & $\mathrm{N} / \mathrm{A}$ \\
\hline $\operatorname{miR}-1011 /+^{\mathrm{D} 7}$ & 5.464 & 2.732 & 0.546 & 183 & 7.349 & - \\
\hline $\operatorname{miR}-1011 /+^{\mathrm{TS}}$ & 6.818 & 5.114 & 2.273 & 176 & 3.634 & $19.635^{* * *}$ \\
\hline
\end{tabular}




\begin{tabular}{|c|c|c|c|c|c|c|}
\hline $\mathrm{miR}-1011 /+^{\mathrm{SS}}$ & 8.333 & 0.269 & - & 108 & 3.412 & 5.172 \\
\hline $\mathrm{miR}-1011 /+^{\mathrm{PS}}$ & 15.657 & 0.926 & - & 198 & $13.974^{* *}$ & $13.513^{* *}$ \\
\hline $\mathrm{miR}-1011 / \mathrm{+}^{\mathrm{D} 30}$ & 5.263 & 4.386 & 0.877 & 114 & 2.854 & $15.489^{* *}$ \\
\hline
\end{tabular}

The frequency of muscle degeneration results was compared using the $\chi^{2}$ test with 3 degrees of freedom and Yate's correction

$\mathrm{n}=$ number of muscles analyzed

${ }^{*} \mathrm{p}<0.05, * * \mathrm{p}<0.01, * * * \mathrm{p}<0.001$ represents comparison to the control at the same condition, whilst $* \mathrm{p}<0.05, * * \mathrm{p}<0.01, * * * \mathrm{p}<0.001$ represents comparison within the same genotype to young age

$\mathrm{D} 7=7$ day old flies, $\mathrm{TS}=$ temperature stress, $\mathrm{SS}=$ sugar starvation, $\mathrm{PS}=$ protein starvation, $\mathrm{D} 30=30$ day old flies 
Supplementary Table 4. Percentage of muscle degeneration in selected miRNA mutants

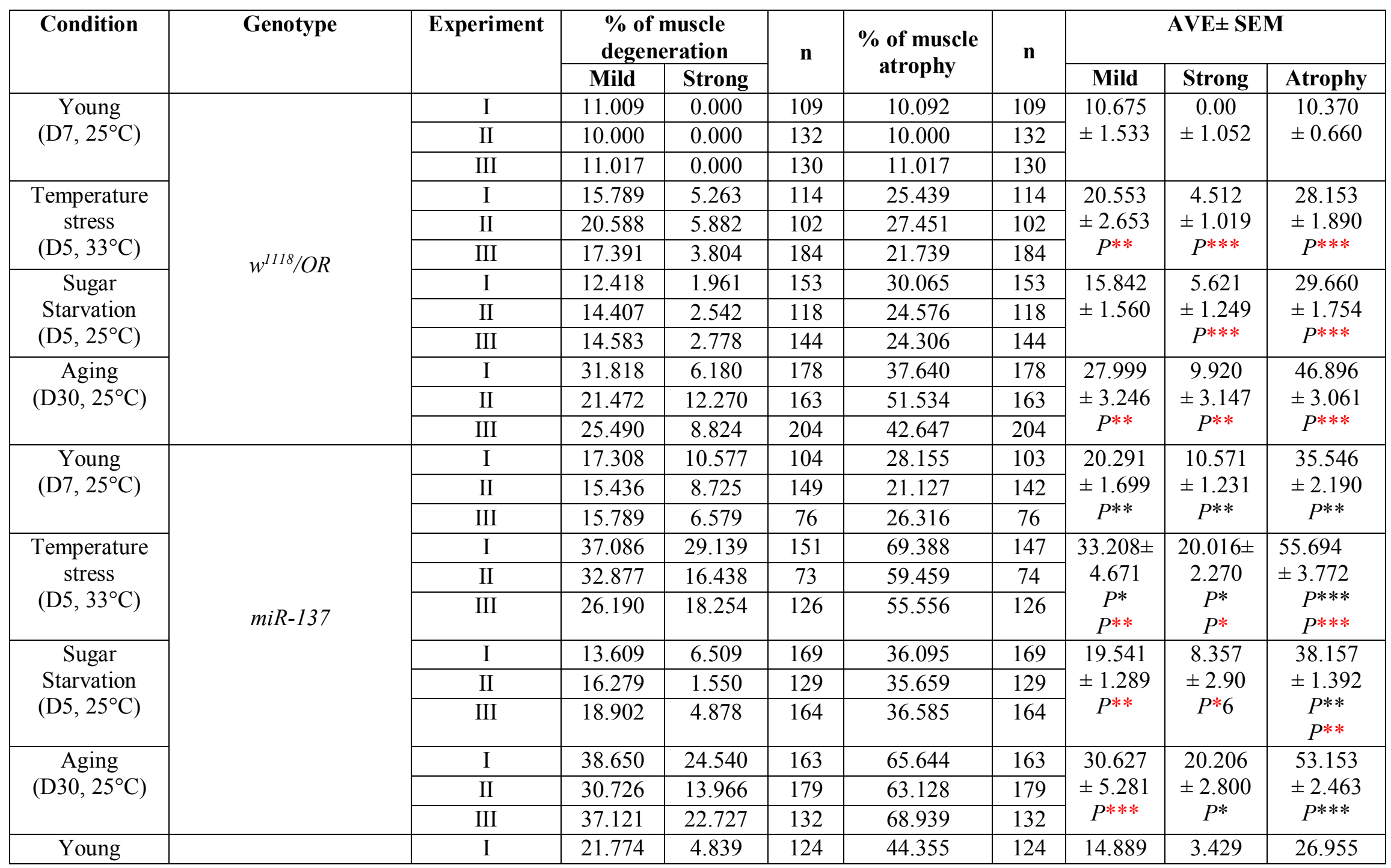




\begin{tabular}{|c|c|c|c|c|c|c|c|c|c|c|}
\hline \multirow[t]{2}{*}{$\left(\mathrm{D} 7,25^{\circ} \mathrm{C}\right)$} & & II & 15.104 & 2.604 & 192 & 30.729 & 192 & \multirow[t]{2}{*}{ \pm 3.532} & \multirow{2}{*}{$\begin{array}{c} \pm 2.782 \\
P^{* *}\end{array}$} & \multirow{2}{*}{$\begin{array}{c} \pm 8.111 \\
P^{*}\end{array}$} \\
\hline & & III & 12.179 & 3.205 & 156 & 19.231 & 156 & & & \\
\hline \multirow{3}{*}{$\begin{array}{l}\text { Temperature } \\
\text { stress } \\
\left(\mathrm{D} 5,33^{\circ} \mathrm{C}\right)\end{array}$} & & I & 17.424 & 5.303 & 132 & 20.455 & 132 & \multirow{3}{*}{$\begin{array}{r}16.178 \\
\pm 3.422\end{array}$} & \multirow{3}{*}{$\begin{array}{c}8.627 \\
\pm 2.839\end{array}$} & \multirow{3}{*}{$\begin{array}{l}31.438 \\
\pm 6.813\end{array}$} \\
\hline & & II & 9.333 & 2.000 & 150 & 17.333 & 150 & & & \\
\hline & & III & 17.910 & 2.985 & 67 & 43.077 & 65 & & & \\
\hline \multirow{3}{*}{$\begin{array}{c}\text { Sugar } \\
\text { Starvation } \\
\left(\mathrm{D} 5,25^{\circ} \mathrm{C}\right)\end{array}$} & & I & 14.167 & 0.833 & 120 & 45.833 & 120 & \multirow{3}{*}{$\begin{array}{c}13.621 \\
\pm 0.355\end{array}$} & \multirow{3}{*}{$\begin{array}{c}1.201 \\
\pm 0.490 \\
P^{*} \\
P^{*}\end{array}$} & \multirow{3}{*}{$\begin{array}{c}40.252 \\
\pm 3.216 \\
P^{*}\end{array}$} \\
\hline & & II & 14.054 & 1.622 & 185 & 34.694 & 196 & & & \\
\hline & \multirow{4}{*}{$m i R-927$} & III & 12.644 & 1.149 & 174 & 40.230 & 174 & & & \\
\hline \multirow{3}{*}{$\begin{array}{c}\text { Aging } \\
\left(\mathrm{D} 30,25^{\circ} \mathrm{C}\right)\end{array}$} & & I & 43.262 & 16.312 & 141 & 57.447 & 141 & \multirow{3}{*}{$\begin{array}{c}45.255 \\
\pm 4.096 \\
P^{*} \\
P^{* *}\end{array}$} & \multirow{3}{*}{$\begin{array}{c}11.845 \\
\pm 2.527 \\
P^{*}\end{array}$} & \multirow{3}{*}{$\begin{array}{c}64.955 \\
\pm 0.951 \\
P^{*} \\
P^{*}\end{array}$} \\
\hline & & II & 53.846 & 12.500 & 104 & 70.192 & 104 & & & \\
\hline & & III & 38.655 & 6.723 & 119 & 67.227 & 119 & & & \\
\hline \multirow{3}{*}{$\begin{array}{c}\text { Young } \\
\left(\mathrm{D} 7,25^{\circ} \mathrm{C}\right)\end{array}$} & \multirow{12}{*}{ miR-966 } & I & 12.717 & 1.734 & 173 & 23.699 & 173 & \multirow{3}{*}{$\begin{array}{c}14.872 \\
\pm 5.385\end{array}$} & \multirow{3}{*}{$\begin{array}{c}3.333 \\
\pm 3.777 \\
P^{* *}\end{array}$} & \multirow{3}{*}{$\begin{array}{c}38.750 \\
\pm 6.285 \\
P^{*}\end{array}$} \\
\hline & & II & 15.179 & 2.679 & 112 & 37.500 & 112 & & & \\
\hline & & III & 17.500 & 1.250 & 80 & 25.000 & 80 & & & \\
\hline \multirow{3}{*}{$\begin{array}{l}\text { Temperature } \\
\text { stress } \\
\left(\mathrm{D} 5,33^{\circ} \mathrm{C}\right)\end{array}$} & & I & 22.308 & 5.385 & 130 & 46.154 & 130 & \multirow{3}{*}{$\begin{array}{c}15.132 \\
\pm 1.310\end{array}$} & \multirow{3}{*}{$\begin{array}{c}1.888 \\
\pm 1.381\end{array}$} & \multirow{3}{*}{$\begin{array}{l}28.733 \\
\pm 4.399\end{array}$} \\
\hline & & II & 12.308 & 4.615 & 130 & 43.846 & 130 & & & \\
\hline & & III & 10.000 & 0.000 & 80 & 26.250 & 80 & & & \\
\hline \multirow{3}{*}{$\begin{array}{c}\text { Sugar } \\
\text { Starvation } \\
\left(\mathrm{D} 5,25^{\circ} \mathrm{C}\right)\end{array}$} & & I & 17.683 & 0.610 & 164 & 42.073 & 164 & \multirow{3}{*}{$\begin{array}{c}6.533 \\
\pm 4.096 \\
P^{*}\end{array}$} & \multirow{3}{*}{$\begin{array}{c}1.730 \\
\pm 2.527 \\
P^{* *} \\
P^{*}\end{array}$} & \multirow{3}{*}{$\begin{array}{c}43.967 \\
\pm 0.951 \\
P^{* *} \\
P^{* *}\end{array}$} \\
\hline & & II & 19.255 & 0.000 & 161 & 44.767 & 172 & & & \\
\hline & & III & 25.926 & 0.617 & 162 & 45.062 & 162 & & & \\
\hline Aging & & I & 48.780 & 31.098 & 164 & 79.268 & 164 & 36.897 & 32.910 & 67.989 \\
\hline$\left(\mathrm{D} 30,25^{\circ} \mathrm{C}\right)$ & & II & 28.049 & 43.293 & 164 & 70.732 & 164 & \pm 6.304 & \pm 6.174 & \pm 7.431 \\
\hline & & III & 33.862 & 24.339 & 189 & 53.968 & 189 & $P^{*}$ & $\begin{array}{c}P^{*} \\
P^{* *}\end{array}$ & $\begin{array}{c}P^{*} \\
P^{* *}\end{array}$ \\
\hline Young & & $\mathrm{I}$ & 14.953 & 2.804 & 107 & 16.822 & 107 & 10.887 & 2.221 & 13.073 \\
\hline$\left(\mathrm{D} 7,25^{\circ} \mathrm{C}\right)$ & & II & 9.375 & 2.344 & 128 & 14.063 & 128 & \pm 2.232 & \pm 2.055 & \pm 2.500 \\
\hline & & III & 8.333 & 1.515 & 132 & 8.333 & 132 & & & \\
\hline Temperature & & I & 20.611 & 1.527 & 131 & 22.727 & 132 & 20.002 & 3.181 & 27.853 \\
\hline stress & & II & 22.727 & 4.545 & 110 & 30.275 & 109 & \pm 2.014 & \pm 1.776 & \pm 2.564 \\
\hline
\end{tabular}




\begin{tabular}{|c|c|c|c|c|c|c|c|c|c|c|}
\hline$\left(\mathrm{D} 5,33^{\circ} \mathrm{C}\right)$ & \multirow{7}{*}{ Mhc-Gal4/+ } & III & 16.667 & 3.472 & 144 & 30.556 & 144 & $P^{* *}$ & & $P^{* *}$ \\
\hline \multirow{3}{*}{$\begin{array}{c}\text { Sugar } \\
\text { Starvation } \\
\left(\mathrm{D} 5,25^{\circ} \mathrm{C}\right)\end{array}$} & & $\mathrm{I}$ & 24.113 & 1.418 & 141 & 33.333 & 141 & \multirow{3}{*}{$\begin{array}{c}19.006 \\
\pm 2.319 \\
P^{*}\end{array}$} & \multirow{3}{*}{$\begin{array}{c}1.969 \\
\pm 2.556\end{array}$} & \multirow{3}{*}{$\begin{array}{c}36.705 \\
\pm 4.330 \\
P^{* *}\end{array}$} \\
\hline & & II & 16.667 & 2.778 & 108 & 31.481 & 108 & & & \\
\hline & & III & 16.239 & 1.709 & 117 & 45.299 & 117 & & & \\
\hline \multirow{3}{*}{$\begin{array}{c}\text { Aging } \\
\left(\mathrm{D} 30,25^{\circ} \mathrm{C}\right)\end{array}$} & & $\mathrm{I}$ & 34.932 & 6.164 & 146 & 45.205 & 146 & \multirow{3}{*}{$\begin{array}{c}32.227 \\
\pm 4.972 \\
P^{* *}\end{array}$} & \multirow{3}{*}{$\begin{array}{c}8.628 \\
\pm 4.280 \\
P^{* *}\end{array}$} & \multirow{3}{*}{$\begin{array}{c}49.220 \\
\pm 8.037 \\
P^{* *}\end{array}$} \\
\hline & & II & 37.908 & 11.111 & 153 & 64.706 & 153 & & & \\
\hline & & III & 23.841 & 8.609 & 151 & 37.748 & 151 & & & \\
\hline \multirow{3}{*}{$\begin{array}{c}\text { Young } \\
\left(\mathrm{D} 7,25^{\circ} \mathrm{C}\right)\end{array}$} & \multirow{12}{*}{$M h c>D g$} & I & 35.938 & 7.031 & 128 & 39.844 & 128 & \multirow{3}{*}{$\begin{array}{c}22.856 \\
\pm 3.158\end{array}$} & \multirow{3}{*}{$\begin{array}{c}4.800 \\
\pm 6.878 \\
P^{*}\end{array}$} & \multirow{3}{*}{$\begin{array}{l}34.334 \\
\pm 1.657\end{array}$} \\
\hline & & II & 12.632 & 4.211 & 95 & 20.000 & 95 & & & \\
\hline & & III & 20.000 & 3.158 & 95 & 43.158 & 95 & & & \\
\hline \multirow{3}{*}{$\begin{array}{l}\text { Temperature } \\
\text { stress } \\
\left(\mathrm{D} 5,33^{\circ} \mathrm{C}\right)\end{array}$} & & I & 35.821 & 10.448 & 134 & 42.857 & 133 & \multirow{3}{*}{$\begin{array}{c}30.977 \\
\pm 5.535 \\
P^{*}\end{array}$} & \multirow{3}{*}{$\begin{array}{c}6.315 \\
\pm 4.858\end{array}$} & \multirow{3}{*}{$\begin{array}{c}39.829 \\
\pm 1.692 \\
P^{*}\end{array}$} \\
\hline & & II & 35.849 & 3.774 & 106 & 39.623 & 106 & & & \\
\hline & & III & 21.260 & 4.724 & 127 & 37.008 & 127 & & & \\
\hline \multirow{3}{*}{$\begin{array}{c}\text { Sugar } \\
\text { Starvation } \\
\left(\mathrm{D} 5,25^{\circ} \mathrm{C}\right)\end{array}$} & & I & 28.846 & 0.000 & 156 & 66.667 & 156 & \multirow{3}{*}{\begin{tabular}{|c|}
22.497 \\
\pm 5.747
\end{tabular}} & \multirow{3}{*}{$\begin{array}{c}0.673 \\
\pm 5.113 \\
P^{*}\end{array}$} & \multirow{3}{*}{$\begin{array}{c}67.619 \\
\pm 11.746 \\
P^{*}\end{array}$} \\
\hline & & II & 26.263 & 2.020 & 99 & 66.667 & 99 & & & \\
\hline & & III & 12.381 & 0.000 & 105 & 31.429 & 105 & & & \\
\hline \multirow{3}{*}{$\begin{array}{c}\text { Aging } \\
\left(\mathrm{D} 30,25^{\circ} \mathrm{C}\right)\end{array}$} & & $\mathrm{I}$ & 37.815 & 21.008 & 119 & 64.706 & 119 & \multirow{3}{*}{$\begin{array}{c}43.057 \\
\pm 0.777 \\
P^{*} \\
\end{array}$} & \multirow{3}{*}{$\begin{array}{c}12.714 \\
\pm 3.498\end{array}$} & \multirow{3}{*}{$\begin{array}{c}59.509 \\
\pm 3.235 \\
P^{*}\end{array}$} \\
\hline & & II & 41.667 & 5.952 & 84 & 53.571 & 84 & & & \\
\hline & & III & 49.689 & 11.180 & 161 & 60.248 & 161 & & & \\
\hline Young & \multirow{12}{*}{$M h c>m i R-137$} & I & \multirow{3}{*}{\multicolumn{5}{|c|}{$\begin{array}{l}\text { Semi-lethal } \\
\text { - } \quad \text { escapers with no muscles } \\
\text { - } \quad \text { escapers had erect wing }\end{array}$}} & - & - & - \\
\hline$\left(\mathrm{D} 7,25^{\circ} \mathrm{C}\right)$ & & II & & & & & & & & \\
\hline & & III & & & & & & & & \\
\hline Temperature & & I & & & emi-le & & & - & - & - \\
\hline stress & & II & & - esc & pers $w$ & o muscles & & & & \\
\hline$\left(\mathrm{D} 5,33^{\circ} \mathrm{C}\right)$ & & III & & - es & apers 1 & rect wing & & & & \\
\hline Sugar & & I & & & emi-le & & & - & - & - \\
\hline Starvation & & II & & - esc & pers $\mathrm{w}$ & o muscles & & & & \\
\hline$\left(\mathrm{D} 5,25^{\circ} \mathrm{C}\right)$ & & III & & $\bullet$ & apers 1 & rect wing & & & & \\
\hline Aging & & I & & & emi-le & 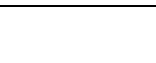 & & - & - & - \\
\hline$\left(\mathrm{D} 30,25^{\circ} \mathrm{C}\right)$ & & II & & - esc & pers $\mathrm{w}$ & muscles & & & & \\
\hline & & III & & & apers 1 & rect wing & & & & \\
\hline
\end{tabular}




\begin{tabular}{|c|c|c|c|c|c|c|c|c|c|c|}
\hline \multirow{3}{*}{$\begin{array}{c}\text { Young } \\
\left(\mathrm{D} 7,25^{\circ} \mathrm{C}\right)\end{array}$} & & $\mathrm{I}$ & 18.493 & 19.178 & 146 & 73.288 & 146 & \multirow{3}{*}{$\begin{array}{c}18.303 \\
\pm 0.883 \\
P^{*}\end{array}$} & \multirow{3}{*}{$\begin{array}{c}15.854 \\
\pm 0.442 \\
P^{* *}\end{array}$} & \multirow{3}{*}{$\begin{array}{c}70.346 \\
\pm 2.163 \\
P^{* * *}\end{array}$} \\
\hline & & II & 18.954 & 15.686 & 153 & 71.622 & 148 & & & \\
\hline & & III & 17.460 & 12.698 & 63 & 66.129 & 62 & & & \\
\hline \multirow{3}{*}{$\begin{array}{c}\text { Temperature } \\
\text { stress } \\
\left(\mathrm{D} 5,33^{\circ} \mathrm{C}\right)\end{array}$} & \multirow{8}{*}{$M h c>m i R-927^{a}$} & I & 39.634 & 23.780 & 164 & 86.806 & 144 & \multirow{3}{*}{$\begin{array}{c}38.583 \\
\pm 4.901 \\
P^{*} \\
P^{*}\end{array}$} & \multirow{3}{*}{$\begin{array}{c}28.191 \\
\pm 6.093 \\
P^{* * *} \\
P^{* *}\end{array}$} & \multirow{3}{*}{$\begin{array}{c}89.343 \\
\pm 1.582 \\
P^{* * *} \\
P^{* * *}\end{array}$} \\
\hline & & II & 27.545 & 32.934 & 167 & 88.976 & 127 & & & \\
\hline & & III & 48.571 & 27.857 & 140 & 92.248 & 129 & & & \\
\hline \multirow{2}{*}{$\begin{array}{c}\text { Sugar } \\
\text { Starvation } \\
\left(\mathrm{D} 5,25^{\circ} \mathrm{C}\right)\end{array}$} & & I & 29.358 & 18.349 & 109 & 82.569 & 109 & \multirow{2}{*}{$\begin{array}{c}33.227 \\
\pm 0.355 \\
P^{*} \\
P^{* *}\end{array}$} & \multirow{2}{*}{$\begin{array}{c}13.207 \\
\pm 3.869\end{array}$} & \multirow{2}{*}{$\begin{array}{c}76.768 \\
\pm 5.801 \\
P^{* *}\end{array}$} \\
\hline & & II & 37.097 & 8.065 & 62 & 70.968 & 62 & & & \\
\hline \multirow{3}{*}{$\begin{array}{c}\text { Aging } \\
\left(\mathrm{D} 30,25^{\circ} \mathrm{C}\right)\end{array}$} & & $\mathrm{I}$ & 47.059 & 58.170 & 153 & 77.124 & 153 & \multirow{3}{*}{\begin{tabular}{|c|}
45.364 \\
\pm 4.818 \\
$P^{* * *}$
\end{tabular}} & \multirow{3}{*}{$\begin{array}{c}39.799 \\
\pm 2.501 \\
P^{*} \\
P^{*}\end{array}$} & \multirow{3}{*}{$\begin{array}{c}77.438 \\
\pm 2.776 \\
P^{*}\end{array}$} \\
\hline & & II & 40.441 & 32.353 & 136 & 72.794 & 136 & & & \\
\hline & & III & 48.592 & 28.873 & 142 & 82.394 & 142 & & & \\
\hline \multirow{3}{*}{$\begin{array}{c}\text { Young } \\
\left(\mathrm{D} 7,25^{\circ} \mathrm{C}\right)\end{array}$} & \multirow{12}{*}{$M h c>m i R-966$} & I & 17.483 & 3.497 & 143 & 26.573 & 143 & \multirow{3}{*}{$\begin{array}{c}19.699 \\
\pm 1.728 \\
P^{*}\end{array}$} & \multirow{3}{*}{$\begin{array}{c}2.010 \\
\pm 1.923\end{array}$} & \multirow{3}{*}{$\begin{array}{c}29.235 \\
\pm 1.404 \\
P^{* *}\end{array}$} \\
\hline & & II & 18.085 & 1.064 & 94 & 29.787 & 94 & & & \\
\hline & & III & 23.529 & 1.471 & 68 & 31.343 & 67 & & & \\
\hline \multirow{3}{*}{$\begin{array}{c}\text { Temperature } \\
\text { stress } \\
\left(\mathrm{D} 5,33^{\circ} \mathrm{C}\right)\end{array}$} & & I & 33.824 & 32.353 & 136 & 95.041 & 121 & \multirow{3}{*}{\begin{tabular}{|r|}
26.230 \\
\pm 9.861 \\
\end{tabular}} & \multirow{3}{*}{$\begin{array}{c}27.072 \\
\pm 3.841 \\
P^{* * *} \\
P^{*}\end{array}$} & \multirow{3}{*}{$\begin{array}{c}79.652 \\
\pm 7.669 \\
P^{* *} \\
P^{* *}\end{array}$} \\
\hline & & II & 23.438 & 36.719 & 128 & 72.381 & 105 & & & \\
\hline & & III & 21.429 & 12.143 & 140 & 71.533 & 137 & & & \\
\hline \multirow{3}{*}{$\begin{array}{c}\text { Sugar } \\
\text { Starvation } \\
\left(\mathrm{D} 5,25^{\circ} \mathrm{C}\right)\end{array}$} & & $\mathrm{I}$ & 31.618 & 12.500 & 136 & 55.882 & 136 & \multirow{3}{*}{$\begin{array}{c}28.791 \\
\pm 3.333 \\
P^{*} \\
P^{* *}\end{array}$} & \multirow{3}{*}{$\begin{array}{c}8.065 \\
\pm 1.422 \\
P^{*} \\
P^{*}\end{array}$} & \multirow{3}{*}{$\begin{array}{c}52.231 \\
\pm 3.776 \\
P^{*} \\
P^{* *}\end{array}$} \\
\hline & & II & 27.660 & 4.965 & 141 & 44.681 & 141 & & & \\
\hline & & III & 27.097 & 10.968 & 155 & 56.129 & 155 & & & \\
\hline Aging & & I & 37.097 & 16.129 & 124 & 69.355 & 124 & \multirow{3}{*}{$\begin{array}{c}40.365 \\
\pm 4.632 \\
P^{* * * *}\end{array}$} & 20.016 & 73.194 \\
\hline$\left(\mathrm{D} 30,25^{\circ} \mathrm{C}\right)$ & & II & 48.148 & 22.222 & 108 & 87.963 & 108 & & \pm 3.908 & \pm 7.663 \\
\hline & & III & 35.849 & 21.698 & 106 & 62.264 & 106 & & $\begin{array}{c}P^{* *} \\
P^{* * *}\end{array}$ & $P^{* *}$ \\
\hline Young & & I & 7.692 & 1.282 & 78 & 23.077 & 78 & 8.022 & 1.152 & 20.730 \\
\hline$\left(\mathrm{D} 7,25^{\circ} \mathrm{C}\right)$ & & II & 7.071 & 1.010 & 99 & 18.182 & 99 & \pm 0.665 & \pm 0.665 & \pm 1.417 \\
\hline & & III & 9.302 & 1.163 & 86 & 20.930 & 86 & & & \\
\hline
\end{tabular}




\begin{tabular}{|c|c|c|c|c|c|c|c|c|c|c|}
\hline \multirow{3}{*}{$\begin{array}{c}\text { Temperature } \\
\text { stress } \\
\left(\mathrm{D} 5,33^{\circ} \mathrm{C}\right) \\
\end{array}$} & \multirow{9}{*}{ Mhc $>$ scramble sponge } & $\mathrm{I}$ & 11.538 & 7.692 & 104 & 35.577 & 104 & \multirow{3}{*}{$\begin{array}{c}14.866 \\
\pm 1.081 \\
P^{*}\end{array}$} & \multirow{3}{*}{$\begin{array}{c}6.374 \\
\pm 2.294 \\
P^{* *}\end{array}$} & \multirow{3}{*}{$\begin{array}{c}31.161 \\
\pm 2.228 \\
P^{* *}\end{array}$} \\
\hline & & II & 13.793 & 7.759 & 116 & 29.464 & 112 & & & \\
\hline & & III & 19.266 & 3.670 & 109 & 28.440 & 109 & & & \\
\hline \multirow{3}{*}{$\begin{array}{c}\text { Sugar } \\
\text { Starvation } \\
\left(\mathrm{D} 5,25^{\circ} \mathrm{C}\right)\end{array}$} & & $\mathrm{I}$ & 8.594 & 1.563 & 128 & 12.500 & 128 & \multirow{3}{*}{$\begin{array}{c}17.268 \\
\pm 4.403 \\
P^{*}\end{array}$} & \multirow{3}{*}{$\begin{array}{c}1.516 \\
\pm 4.337\end{array}$} & \multirow{3}{*}{$\begin{array}{l}17.619 \\
\pm 4.997\end{array}$} \\
\hline & & II & 21.642 & 2.985 & 134 & 27.612 & 134 & & & \\
\hline & & III & 21.569 & 0.000 & 102 & 12.745 & 102 & & & \\
\hline \multirow{3}{*}{$\begin{array}{c}\text { Aging } \\
\left(\mathrm{D} 30,25^{\circ} \mathrm{C}\right)\end{array}$} & & I & 20.492 & 7.377 & 122 & 69.672 & 122 & \multirow{3}{*}{$\begin{array}{c}30.441 \\
\pm 6.712 \\
P^{* *}\end{array}$} & \multirow{3}{*}{$\begin{array}{c}9.787 \\
\pm 5.191 \\
P^{* *}\end{array}$} & \multirow{3}{*}{$\begin{array}{c}71.722 \\
\pm 3.792 \\
P^{* * *}\end{array}$} \\
\hline & & II & 32.847 & 8.029 & 137 & 66.423 & 137 & & & \\
\hline & & III & 37.984 & 13.953 & 129 & 79.070 & 129 & & & \\
\hline \multirow{3}{*}{$\begin{array}{c}\text { Young } \\
\left(\mathrm{D} 7,25^{\circ} \mathrm{C}\right)\end{array}$} & \multirow{12}{*}{$M h c>m i R-137^{\text {sponge }}$} & $\mathrm{I}$ & 20.000 & 3.571 & 140 & 21.429 & 140 & \multirow{3}{*}{$\begin{array}{c}24.938 \\
\pm 3.536 \\
P^{* *}\end{array}$} & \multirow{3}{*}{$\begin{array}{c}4.724 \\
\pm 3.356 \\
P^{* *}\end{array}$} & \multirow{3}{*}{$\begin{array}{l}26.294 \\
\pm 4.394\end{array}$} \\
\hline & & II & 23.469 & 6.122 & 98 & 35.065 & 77 & & & \\
\hline & & III & 31.343 & 4.478 & 67 & 22.388 & 67 & & & \\
\hline \multirow{3}{*}{$\begin{array}{c}\text { Temperature } \\
\text { stress } \\
\left(\mathrm{D} 5,33^{\circ} \mathrm{C}\right)\end{array}$} & & I & 24.719 & 8.989 & 89 & 71.910 & 89 & \multirow{3}{*}{$\begin{array}{c}25.644 \\
\pm 1.081 \\
P^{* *}\end{array}$} & \multirow{3}{*}{$\begin{array}{c}8.601 \\
\pm 2.294 \\
P^{* *}\end{array}$} & \multirow{3}{*}{$\begin{array}{c}68.684 \\
\pm 2.228 \\
P^{* *} \\
P^{* *}\end{array}$} \\
\hline & & II & 24.779 & 8.850 & 113 & 58.036 & 112 & & & \\
\hline & & III & 27.434 & 7.965 & 113 & 76.106 & 113 & & & \\
\hline \multirow{3}{*}{$\begin{array}{c}\text { Sugar } \\
\text { Starvation } \\
\left(\mathrm{D} 5,25^{\circ} \mathrm{C}\right)\end{array}$} & & $\mathrm{I}$ & 28.472 & 13.194 & 144 & 63.194 & 144 & \multirow{3}{*}{$\begin{array}{c}31.420 \\
\pm 2.563 \\
P^{*}\end{array}$} & \multirow{3}{*}{$\begin{array}{c}10.317 \\
\pm 2.851 \\
P^{* *} \\
P^{* *}\end{array}$} & \multirow{3}{*}{$\begin{array}{c}60.550 \\
\pm 6.616 \\
P^{* *} \\
P^{* *}\end{array}$} \\
\hline & & II & 37.121 & 9.091 & 132 & 70.455 & 132 & & & \\
\hline & & III & 28.667 & 8.667 & 150 & 48.000 & 150 & & & \\
\hline & & I & 38.462 & 23.846 & 130 & 60.656 & 122 & \multirow{3}{*}{$\begin{array}{c}37.618 \\
\pm 4.776 \\
P^{* *}\end{array}$} & \multirow{3}{*}{$\begin{array}{c}27.169 \\
\pm 1.337 \\
P^{* *} \\
P^{* *}\end{array}$} & \multirow{3}{*}{$\begin{array}{c}67.643 \\
\pm 4.976 \\
P^{* *}\end{array}$} \\
\hline$\left(\mathrm{D} 30,25^{\circ} \mathrm{C}\right)$ & & II & 39.394 & 34.091 & 132 & 77.273 & 132 & & & \\
\hline & & III & 35.000 & 23.571 & 140 & 65.000 & 140 & & & \\
\hline Young & & I & 9.649 & 1.754 & 114 & 17.544 & 114 & 9.524 & 1.385 & 19.725 \\
\hline$\left(\mathrm{D} 7,25^{\circ} \mathrm{C}\right)$ & & II & 6.923 & 0.000 & 130 & 19.231 & 130 & \pm 1.773 & \pm 1.467 & \pm 1.423 \\
\hline & & III & 12.000 & 2.400 & 125 & 22.400 & 125 & & & \\
\hline Temperature & & I & 17.714 & 1.714 & 175 & 32.571 & 175 & 17.491 & 1.748 & 37.029 \\
\hline stress & & II & 17.518 & 1.460 & 137 & 37.956 & 137 & \pm 0.618 & \pm 0.137 & \pm 2.352 \\
\hline$\left(\mathrm{D} 5,33^{\circ} \mathrm{C}\right)$ & & III & 17.241 & 2.069 & 145 & 40.559 & 143 & & & $P^{* *}$ \\
\hline Sugar & how-Gal4/+ & $\mathrm{I}$ & 13.907 & 4.636 & 151 & 21.854 & 151 & 12.840 & 3.505 & 25.028 \\
\hline Starvation & & II & 8.333 & 2.778 & 180 & 22.222 & 180 & \pm 2.628 & \pm 2.355 & \pm 2.992 \\
\hline
\end{tabular}




\begin{tabular}{|c|c|c|c|c|c|c|c|c|c|c|}
\hline$\left(\mathrm{D} 5,25^{\circ} \mathrm{C}\right)$ & & III & 16.279 & 3.101 & 129 & 31.008 & 129 & & $P^{*}$ & \\
\hline \multirow{3}{*}{$\begin{array}{c}\text { Aging } \\
\left(\mathrm{D} 30,25^{\circ} \mathrm{C}\right)\end{array}$} & & I & 32.639 & 8.333 & 144 & 40.972 & 144 & \multirow{3}{*}{$\begin{array}{c}31.484 \\
\pm 0.537 \\
P^{* * *}\end{array}$} & \multirow{3}{*}{$\begin{array}{c}9.667 \\
\pm 1.807 \\
P^{* *}\end{array}$} & \multirow{3}{*}{$\begin{array}{c}47.212 \\
\pm 3.188 \\
P^{* * *}\end{array}$} \\
\hline & & II & 27.941 & 14.216 & 204 & 51.471 & 204 & & & \\
\hline & & III & 33.871 & 6.452 & 124 & 49.194 & 124 & & & \\
\hline $\begin{array}{c}\text { Day } 7 \\
\left(\mathrm{D} 7,25^{\circ} \mathrm{C}\right)\end{array}$ & how $>D g$ & I & \multicolumn{5}{|c|}{ Semi-lethal (escapers with fused muscle) } & - & - & - \\
\hline $\begin{array}{c}\text { Day } 7 \\
\left(\mathrm{D} 7,25^{\circ} \mathrm{C}\right)\end{array}$ & how $>m i R-137$ & II & \multicolumn{5}{|c|}{ Embryonic lethal } & - & - & - \\
\hline $\begin{array}{c}\text { Day } 7 \\
\left(\mathrm{D} 7,25^{\circ} \mathrm{C}\right)\end{array}$ & how $>$ miR-927 & III & \multicolumn{5}{|c|}{ Pupal lethal } & - & - & - \\
\hline \multirow{3}{*}{$\begin{array}{c}\text { Young } \\
\left(\mathrm{D} 7,25^{\circ} \mathrm{C}\right)\end{array}$} & \multirow{10}{*}{ how $>m i R-966$} & I & 18.056 & 0.000 & 72 & 13.889 & 72 & \multirow{3}{*}{$\begin{array}{c}20.741 \\
\pm 2.381 \\
P^{* *}\end{array}$} & \multirow{3}{*}{$\begin{array}{c}1.852 \\
\pm 1.606\end{array}$} & \multirow{3}{*}{$\begin{array}{l}21.019 \\
\pm 4.171\end{array}$} \\
\hline & & II & 23.611 & 0.000 & 72 & 20.833 & 72 & & & \\
\hline & & III & 20.556 & 5.556 & 180 & 28.333 & 180 & & & \\
\hline \multirow{3}{*}{$\begin{array}{l}\text { Temperature } \\
\text { stress } \\
\left(\mathrm{D} 5,33^{\circ} \mathrm{C}\right)\end{array}$} & & I & 12.308 & 2.308 & 130 & 26.923 & 130 & \multirow{3}{*}{$\begin{array}{l}16.678 \\
\pm 3.962\end{array}$} & \multirow{3}{*}{$\begin{array}{c}3.396 \\
\pm 2.922\end{array}$} & \multirow{3}{*}{$\begin{array}{c}30.622 \\
\pm 3.220 \\
P^{*}\end{array}$} \\
\hline & & II & 22.222 & 5.556 & 54 & 37.037 & 54 & & & \\
\hline & & III & 15.504 & 2.326 & 129 & 27.907 & 129 & & & \\
\hline \multirow{2}{*}{$\begin{array}{c}\text { Sugar } \\
\text { Starvation } \\
\left(\mathrm{D} 5,25^{\circ} \mathrm{C}\right)\end{array}$} & & I & 20.313 & 0.000 & 64 & 45.313 & 64 & \multirow{2}{*}{$\begin{array}{c}19.633 \\
\pm 1.608 \\
P^{*}\end{array}$} & \multirow{2}{*}{$\begin{array}{c}2.288 \\
\pm 0.697 \\
P^{* *} \\
P^{* *}\end{array}$} & \multirow{2}{*}{$\begin{array}{c}35.401 \\
\pm 9.911 \\
P^{* *}\end{array}$} \\
\hline & & II & 18.954 & 4.575 & 153 & 25.490 & 153 & & & \\
\hline \multirow{2}{*}{$\begin{array}{c}\text { Aging } \\
\left(\mathrm{D} 30,25^{\circ} \mathrm{C}\right)\end{array}$} & & I & 47.436 & 23.718 & 156 & 69.231 & 156 & \multirow{2}{*}{$\begin{array}{l}40.385 \\
\pm 10.18\end{array}$} & \multirow{2}{*}{$\begin{array}{r}20.986 \\
\pm 5.101\end{array}$} & \multirow{2}{*}{$\begin{array}{r}62.393 \\
\pm 6.838\end{array}$} \\
\hline & & II & 33.333 & 18.254 & 126 & 55.556 & 126 & & & \\
\hline \multirow{3}{*}{$\begin{array}{c}\text { Young } \\
\left(\mathrm{D} 7,25^{\circ} \mathrm{C}\right)\end{array}$} & & $\mathrm{I}$ & 14.365 & 1.657 & 181 & 23.316 & 193 & \multirow{3}{*}{$\begin{array}{c}13.333 \\
\pm 1.003\end{array}$} & \multirow{3}{*}{$\begin{array}{c}1.306 \\
\pm 0.633\end{array}$} & \multirow{3}{*}{$\begin{array}{l}25.179 \\
\pm 2.677\end{array}$} \\
\hline & & II & 12.183 & 0.508 & 197 & 30.457 & 197 & & & \\
\hline & & III & 13.450 & 1.754 & 171 & 21.765 & 170 & & & \\
\hline \multirow{3}{*}{$\begin{array}{l}\text { Temperature } \\
\text { stress } \\
\left(\mathrm{D} 5,33^{\circ} \mathrm{C}\right)\end{array}$} & & I & 11.364 & 1.515 & 132 & 18.182 & 132 & \multirow{3}{*}{$\begin{array}{c}14.492 \\
\pm 1.915\end{array}$} & & 17.982 \\
\hline & & II & 13.281 & 2.344 & 128 & 19.531 & 128 & & \pm 2.230 & \pm 0.957 \\
\hline & how $>$ scramblesponge & III & 18.831 & 0.649 & 154 & 16.234 & 154 & & & $P^{*}$ \\
\hline Sugar & now $>$ scramble & I & 17.886 & 3.252 & 123 & 16.260 & 123 & 18.772 & 2.487 & 18.418 \\
\hline Starvation & & II & 18.788 & 2.424 & 165 & 16.970 & 165 & \pm 0.087 & \pm 0.507 & \pm 1.815 \\
\hline$\left(\mathrm{D} 5,25^{\circ} \mathrm{C}\right)$ & & III & 19.643 & 1.786 & 168 & 22.024 & 168 & $P^{* * *}$ & & $P^{*}$ \\
\hline Aging & & I & 27.586 & 9.483 & 116 & 54.310 & 116 & 26.368 & 7.856 & 54.617 \\
\hline
\end{tabular}




\begin{tabular}{|c|c|c|c|c|c|c|c|c|c|c|}
\hline \multirow[t]{2}{*}{$\left(\mathrm{D} 30,25^{\circ} \mathrm{C}\right)$} & & II & 25.203 & 7.317 & 123 & 56.911 & 123 & \multirow{2}{*}{$\begin{array}{c} \pm 1.471 \\
P^{* * *}\end{array}$} & \multirow[t]{2}{*}{ \pm 0.688} & \multirow[t]{2}{*}{ \pm 1.245} \\
\hline & & III & 26.316 & 6.767 & 133 & 52.632 & 133 & & & \\
\hline \multirow{3}{*}{$\begin{array}{c}\text { Young } \\
\left(\mathrm{D} 7,25^{\circ} \mathrm{C}\right)\end{array}$} & \multirow{12}{*}{ how $>m i R-137^{\text {sponge }}$} & I & 17.606 & 2.817 & 142 & 30.282 & 142 & \multirow{3}{*}{$\begin{array}{c}18.928 \\
\pm 1.003 \\
P^{*}\end{array}$} & \multirow{3}{*}{$\begin{array}{c}2.463 \\
\pm 0.633\end{array}$} & \multirow{3}{*}{$\begin{array}{l}28.221 \\
\pm 2.667\end{array}$} \\
\hline & & II & 16.892 & 0.000 & 148 & 20.667 & 150 & & & \\
\hline & & III & 22.286 & 4.571 & 175 & 33.714 & 175 & & & \\
\hline \multirow{3}{*}{$\begin{array}{c}\text { Temperature } \\
\text { stress } \\
\left(\mathrm{D} 5,33^{\circ} \mathrm{C}\right)\end{array}$} & & I & 24.211 & 8.947 & 190 & 58.201 & 189 & \multirow{3}{*}{$\begin{array}{c}24.719 \\
\pm 3.802 \\
P^{*} \\
P^{*}\end{array}$} & \multirow{3}{*}{$\begin{array}{c}7.199 \\
\pm 1.964 \\
P^{*}\end{array}$} & \multirow{3}{*}{$\begin{array}{c}51.826 \\
\pm 7.126 \\
P^{* *}\end{array}$} \\
\hline & & II & 28.346 & 9.449 & 127 & 59.677 & 124 & & & \\
\hline & & III & 21.600 & 3.200 & 125 & 37.600 & 125 & & & \\
\hline \multirow{3}{*}{$\begin{array}{c}\text { Sugar } \\
\text { Starvation } \\
\left(\mathrm{D} 5,25^{\circ} \mathrm{C}\right)\end{array}$} & & $\mathrm{I}$ & 20.968 & 6.452 & 124 & 29.839 & 124 & \multirow{3}{*}{$\begin{array}{c}22.420 \\
\pm 1.080 \\
P^{*}\end{array}$} & \multirow{3}{*}{$\begin{array}{c}4.995 \\
\pm 0.752\end{array}$} & \multirow{3}{*}{$\begin{array}{c}23.792 \\
\pm 3.034\end{array}$} \\
\hline & & II & 23.485 & 1.515 & 132 & 21.212 & 132 & & & \\
\hline & & III & 22.807 & 7.018 & 114 & 20.325 & 123 & & & \\
\hline \multirow{3}{*}{$\begin{array}{c}\text { Aging } \\
\left(\mathrm{D} 30,25^{\circ} \mathrm{C}\right)\end{array}$} & & $\mathrm{I}$ & 43.636 & 12.727 & 165 & 38.182 & 165 & \multirow{3}{*}{$\begin{array}{c}38.089 \\
\pm 2.871 \\
P^{*} \\
P^{* *} \\
\end{array}$} & \multirow{3}{*}{$\begin{array}{c}15.584 \\
\pm 3.000 \\
P^{*}\end{array}$} & \multirow{3}{*}{$\begin{array}{l}46.548 \\
\pm 4.191 \\
P^{*}\end{array}$} \\
\hline & & II & 37.297 & 10.811 & 185 & 50.270 & 185 & & & \\
\hline & & III & 33.333 & 23.214 & 168 & 51.190 & 168 & & & \\
\hline & & & & & & & & & & \\
\hline \multirow{2}{*}{$\begin{array}{c}\text { Young } \\
\left(\mathrm{D} 7,25^{\circ} \mathrm{C}\right)\end{array}$} & \multirow{10}{*}{$w^{1118} / O R$} & I & 11.538 & 1.923 & 156 & 5.128 & 156 & \multirow{2}{*}{$\begin{array}{c}21.241 \\
\pm 3.289\end{array}$} & \multirow{2}{*}{$\begin{array}{c}4.041 \\
\pm 0.188\end{array}$} & \multirow{2}{*}{$\begin{array}{c}5.064 \\
\pm 0.064\end{array}$} \\
\hline & & II & 10.833 & 0.833 & 120 & 5.000 & 120 & & & \\
\hline \multirow{2}{*}{$\begin{array}{c}\text { Temperature } \\
\text { stress } \\
\left(\mathrm{D} 5,33^{\circ} \mathrm{C}\right) \\
\end{array}$} & & $\mathrm{I}$ & 21.428 & 3.571 & 168 & 32.317 & 164 & \multirow{2}{*}{$\begin{array}{c}23.183 \\
\pm 3.728\end{array}$} & & \multirow{2}{*}{$\begin{array}{c}30.820 \\
\pm 1.497 \\
P^{* *}\end{array}$} \\
\hline & & II & 21.053 & 4.511 & 133 & 29.323 & 133 & & $\begin{array}{c} \pm 0.627 \\
P^{*}\end{array}$ & \\
\hline Sugar & & I & 20.513 & 7.692 & 156 & 32.692 & 156 & 17.882 & 8.815 & 39.088 \\
\hline Starvation & & II & 14.428 & 7.960 & 201 & 30.348 & 201 & \pm 2.186 & \pm 1.804 & \pm 2.398 \\
\hline$\left(\mathrm{D} 5,25^{\circ} \mathrm{C}\right)$ & & III & 18.705 & 10.791 & 139 & 35.971 & 139 & & $P^{* *}$ & $P^{* * *}$ \\
\hline Aging & & I & 22.513 & 6.283 & 191 & 36.126 & 191 & $27.867 \pm$ & 9.871 & 49.852 \\
\hline$\left(\mathrm{D} 30,25^{\circ} \mathrm{C}\right)$ & & II & 30.952 & 10.317 & 252 & 37.302 & 252 & 4.674 & \pm 2.688 & \pm 2.060 \\
\hline & & III & 30.137 & 13.014 & 146 & 43.836 & 146 & $P^{*}$ & $P^{* *}$ & $P^{* * *}$ \\
\hline Young & & I & 25.676 & 12.838 & 148 & 40.541 & 148 & 24.404 & 12.516 & 37.207 \\
\hline$\left(\mathrm{D} 7,25^{\circ} \mathrm{C}\right)$ & & II & 20.690 & 13.300 & 203 & 33.498 & 203 & \pm 1.467 & \pm 1.887 & \pm 2.042 \\
\hline & & III & 26.846 & 11.409 & 149 & 37.584 & 149 & & $P^{* * *}$ & $P^{* * *}$ \\
\hline & & I & 31.847 & 17.197 & 157 & 47.134 & 157 & 34.364 & 18.755 & 49.921 \\
\hline
\end{tabular}




\begin{tabular}{|c|c|c|c|c|c|c|c|c|c|c|}
\hline \multirow{2}{*}{$\begin{array}{c}\text { Temperature } \\
\text { stress } \\
\left(\mathrm{D} 5,33^{\circ} \mathrm{C}\right)\end{array}$} & \multirow{8}{*}{$m i R-137^{k o}$} & II & 36.552 & 21.379 & 145 & 56.738 & 141 & \multirow{2}{*}{$\begin{array}{c} \pm 2.806 \\
P^{* *} \\
P^{* *} \\
\end{array}$} & \multirow{2}{*}{$\begin{array}{c} \pm 1.368 \\
P^{* *} \\
P^{* *}\end{array}$} & \multirow{2}{*}{$\begin{array}{c} \pm 3.427 \\
P^{*}\end{array}$} \\
\hline & & III & 34.694 & 17.687 & 147 & 45.890 & 146 & & & \\
\hline \multirow{3}{*}{$\begin{array}{c}\text { Sugar } \\
\text { Starvation } \\
\left(\mathrm{D} 5,25^{\circ} \mathrm{C}\right)\end{array}$} & & I & 26.389 & 10.417 & 144 & 48.6111 & 144 & \multirow{3}{*}{$\begin{array}{l}22.817 \\
\pm 1.031\end{array}$} & \multirow{3}{*}{$\begin{array}{c}12.401 \\
\pm 4.285\end{array}$} & \multirow{3}{*}{$\begin{array}{l}44.367 \\
\pm 6.333\end{array}$} \\
\hline & & II & 27.778 & 12.500 & 144 & 49.306 & 144 & & & \\
\hline & & III & 14.286 & 14.286 & 119 & 35.185 & 108 & & & \\
\hline \multirow{3}{*}{$\begin{array}{c}\text { Aging } \\
\left(\mathrm{D} 30,25^{\circ} \mathrm{C}\right)\end{array}$} & & $\mathrm{I}$ & 19.444 & 16.667 & 36 & 47.222 & 36 & \multirow{3}{*}{$\begin{array}{l}25.755 \\
\pm 5.324\end{array}$} & \multirow{3}{*}{$\begin{array}{c}20.000 \\
\pm 3.173 \\
P^{*} \\
P^{*}\end{array}$} & \multirow{3}{*}{$\begin{array}{l}52.489 \\
\pm 3.173\end{array}$} \\
\hline & & II & 29.487 & 25.000 & 156 & 51.079 & 139 & & & \\
\hline & & III & 28.333 & 18.333 & 120 & 59.167 & 120 & & & \\
\hline \multirow{3}{*}{$\begin{array}{c}\text { Young } \\
\left(\mathrm{D} 7,25^{\circ} \mathrm{C}\right)\end{array}$} & \multirow{11}{*}{$m i R-137^{k o / D f}$} & I & 16.326 & 11.224 & 98 & 30.612 & 98 & \multirow{3}{*}{$\begin{array}{c}20.765 \pm \\
3.382 \\
P^{* *}\end{array}$} & \multirow{3}{*}{$\begin{array}{c}10.794 \pm \\
2.220 \\
P^{* *}\end{array}$} & \multirow{3}{*}{$\begin{array}{c}27.689 \\
\pm 3.002 \\
P^{* * *}\end{array}$} \\
\hline & & II & 23.077 & 5.495 & 182 & 30.769 & 182 & & & \\
\hline & & III & 22.892 & 15.663 & 166 & 21.687 & 166 & & & \\
\hline \multirow{3}{*}{$\begin{array}{c}\text { Temperature } \\
\text { stress } \\
\left(\mathrm{D} 5,33^{\circ} \mathrm{C}\right)\end{array}$} & & I & 16.667 & 8.333 & 96 & 34.375 & 96 & \multirow{3}{*}{$\begin{array}{l}20.046 \\
\pm 2.650\end{array}$} & \multirow{3}{*}{$\begin{array}{c}9.398 \\
\pm 1.925 \\
P^{* * *}\end{array}$} & \multirow{3}{*}{$\begin{array}{l}39.038 \\
\pm 3.856\end{array}$} \\
\hline & & II & 20.139 & 9.028 & 144 & 38.571 & 140 & & & \\
\hline & & III & 23.333 & 10.833 & 120 & 44.167 & 120 & & & \\
\hline \multirow{3}{*}{$\begin{array}{c}\text { Sugar } \\
\text { Starvation } \\
\left(\mathrm{D} 5,25^{\circ} \mathrm{C}\right)\end{array}$} & & I & 26.316 & 6.015 & 133 & 39.850 & 133 & \multirow{3}{*}{$\begin{array}{c}24.649 \\
\pm 1.489 \\
P^{* *}\end{array}$} & \multirow{3}{*}{$\begin{array}{c}5.831 \\
\pm 1.913\end{array}$} & \multirow{3}{*}{$\begin{array}{l}41.592 \\
\pm 1.145\end{array}$} \\
\hline & & II & 26.797 & 5.229 & 153 & 41.177 & 153 & & & \\
\hline & & III & 20.833 & 6.250 & 48 & 43.750 & 48 & & & \\
\hline \multirow{2}{*}{$\begin{array}{c}\text { Aging } \\
\left(\mathrm{D} 30,25^{\circ} \mathrm{C}\right)\end{array}$} & & I & 33.898 & 8.475 & 112 & 44.915 & 118 & \multirow{2}{*}{$\begin{array}{r}34.137 \\
\pm 1.209 \\
\end{array}$} & \multirow{2}{*}{$\begin{array}{c}9.446 \\
\pm 0.238 \\
\end{array}$} & 46.416 \\
\hline & & II & 34.375 & 10.417 & 96 & 47.917 & 96 & & & \pm 1.501 \\
\hline & & & & & & & & & & \\
\hline Young & & I & 12.698 & 1.587 & 126 & 16.667 & 126 & 14.031 & 0.529 & 18.005 \\
\hline$\left(\mathrm{D} 7,25^{\circ} \mathrm{C}\right)$ & Mhc-Gal4/+ & II & 14.394 & 0.000 & 132 & 18.182 & 132 & \pm 0.513 & \pm 0.407 & \pm 0.515 \\
\hline & & III & 15.000 & 0.000 & 120 & 19.167 & 120 & & & \\
\hline Aging & & I & 16.197 & 5.634 & 142 & 35.915 & 142 & 23.102 & 6.101 & 39.731 \\
\hline$\left(\mathrm{D} 30,25^{\circ} \mathrm{C}\right)$ & Mhc-Gal4/+ & II & 28.916 & 7.831 & 166 & 46.988 & 166 & \pm 2.658 & \pm 0.666 & \pm 2.793 \\
\hline & & III & 24.194 & 4.839 & 124 & 43.046 & 124 & & & ** \\
\hline Young & & I & 29.286 & 5.000 & 151 & 34.416 & 151 & 28.680 & 3.528 & 35.518 \\
\hline$\left(\mathrm{D} 7,25^{\circ} \mathrm{C}\right)$ & $M h c>D g^{R N A i}$ & II & 28.571 & 1.948 & 154 & 29.091 & 154 & \pm 0.233 & \pm 0.608 & \pm 2.898 \\
\hline & & III & 28.182 & 3.636 & 110 & 43.046 & 110 & & & $P^{* *}$ \\
\hline
\end{tabular}




\begin{tabular}{|c|c|c|c|c|c|c|c|c|c|c|}
\hline Aging & & I & 34.409 & 23.118 & 186 & 77.957 & 186 & 33.373 & 18.530 & 67.781 \\
$\left(\mathrm{D} 30,25^{\circ} \mathrm{C}\right)$ & \multirow{3}{*}{$M h c>D g^{R N A i}$} & $\mathrm{II}$ & 33.113 & 12.583 & 151 & 63.758 & 149 & \pm 0.399 & \pm 2.289 & \pm 3.917 \\
& & III & 32.597 & 19.890 & 181 & 61.628 & 172 & $P^{*}$ & $P^{*}$ & $P^{* *}$ \\
& & & & & & & $P^{* *}$ & $P^{* *}$ & $P^{* *}$ \\
\cline { 3 - 11 }
\end{tabular}

Values were obtained from the averages of 3 biological replicates. Error bars represent AVE \pm SEM and statistical significance was determin ed by two-tailed Student's t-test. $* \mathrm{P}<0.05, * * \mathrm{P}<0.01, * * * \mathrm{P}<0.001$ represents comparison to control at the same condition, while $* \mathrm{P}<0.05, * * \mathrm{P}<0.01$, $* * * \mathrm{P}<0.001$ represents comparison within the same genotype at young age.

$\mathrm{n}=$ number of muscles analyzed

a $(M h c>m i R-927)$ male flies with erect wing phenotype 
Supplementary Table 5. Candidate miRNAs can target $D g$ in vitro

\begin{tabular}{|c|c|c|}
\hline $\begin{array}{l}\text { Dg-3'UTR } \\
\text { Reporter }\end{array}$ & $\begin{array}{c}\text { Luciferase Signal (Renilla/Firefly) } \\
\text { AVE } \pm \text { SEM }\end{array}$ & $\begin{array}{c}\text { Relative Luciferase Signal } \\
\mathrm{AVE} \pm \mathrm{SEM}\end{array}$ \\
\hline \multicolumn{3}{|c|}{ Plate 1} \\
\hline $\mathrm{S} 3+m i R-137$ & $1.082 \pm 0.039$ & $1.000 \pm 0.036$ \\
\hline $\mathrm{S} 3+m i R-956$ & $0.129 \pm 0.007$ & $\begin{array}{l}0.499 \pm 0.018 \\
P=5.907 \mathrm{E}-02\end{array}$ \\
\hline $\mathrm{S} 2+m i R-137$ & $0.163 \pm 0.021$ & $\begin{array}{c}0.151 \pm 0.018 \\
P=3.0628 \mathrm{E}-05\end{array}$ \\
\hline \multicolumn{3}{|c|}{ Plate 2} \\
\hline Control & $0.258 \pm 0.006$ & $1.000 \pm 0.022$ \\
\hline $\mathrm{S} 2+m i R-927$ & $0.129 \pm 0.007$ & $\begin{array}{c}0.499 \pm 0.018 \\
P=2.68604 \mathrm{E}-06\end{array}$ \\
\hline Control & $0.209 \pm 0.010$ & $1.000 \pm 0.049$ \\
\hline $\mathrm{S} 2+m i R-966$ & $0.0798 \pm 0.006$ & $\begin{array}{c}0.383 \pm 0.033 \\
P=4.43802 \mathrm{E}-05\end{array}$ \\
\hline Control & $0.211 \pm 0.012$ & $1.000 \pm 0.056$ \\
\hline $\mathrm{S} 1+m i R-137$ & $0.093 \pm 0.006$ & $\begin{array}{l}0.443 \pm 0.032 \\
P=1.361 \mathrm{E}-04\end{array}$ \\
\hline
\end{tabular}

Values obtained for averages of three biological replicates. Error bars represent AVE $\pm \mathrm{SEM}$, and statistical significance was determined by two-tailed Student's t-test $(* \mathrm{P}<0.05$, $* * \mathrm{P}<0.01, * * * \mathrm{P}<0.001)$.

Control readings from respective plates were used to normalize the luciferase signals. 
Supplementary Table 6. Relative $D g$ mRNA levels in adult fly muscle and testes

\begin{tabular}{|c|c|c|c|c|c|c|}
\hline & Genotype & $\begin{array}{c}\operatorname{Rpl}_{32 \mathrm{C}_{\mathrm{T}}} \\
\mathrm{AVE} \pm \mathrm{SEM}\end{array}$ & $\begin{array}{c}D g C_{T} \\
A V E \pm S E M\end{array}$ & $\begin{array}{c}\Delta \mathbf{C}_{\mathbf{T}} \\
\mathrm{AVE} \pm \mathrm{SEM}\end{array}$ & $\begin{array}{c}\text { Average } \\
\Delta \Delta C_{T}\end{array}$ & $\begin{array}{c}\text { Relative mRNA level } \\
\text { AVE } \pm \text { SEM }\end{array}$ \\
\hline \multirow{9}{*}{ 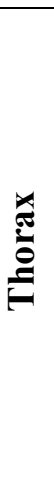 } & \multirow{3}{*}{$w^{1118} / O R$} & $1.64 \mathrm{E}+01 \pm 3.06 \mathrm{E}-01$ & $2.11 \mathrm{E}+01 \pm 1.11 \mathrm{E}-01$ & $4.62 \pm 1.7 \mathrm{E}-01$ & 0.93 & \multirow{3}{*}{$1.000 \pm 5.3 \mathrm{E}-02$} \\
\hline & & $1.68 \mathrm{E}+01 \pm 2.5 \mathrm{E}-02$ & $2.11 \mathrm{E}+01 \pm 4.9 \mathrm{E}-02$ & $4.25 \pm 9.9 \mathrm{E}-02$ & 1.09 & \\
\hline & & $2.24 \mathrm{E}+01 \pm 4.41-02$ & $2.88 \mathrm{E}+01 \pm 5.82 \mathrm{E}-02$ & $6.48 \pm 4.0 \mathrm{E}-02$ & 0.93 & \\
\hline & \multirow{3}{*}{$m i R-137^{k o}$} & $1.79 \mathrm{E}+01 \pm 2.06 \mathrm{E}-02$ & $2.11 \mathrm{E}+01 \pm 4.9 \mathrm{E}-02$ & $3.25 \pm 1.3 \mathrm{E}-02$ & -1.37 & \multirow{3}{*}{$\begin{array}{c}2.97 \pm 8.0 \mathrm{E}-02 \\
P=7.121 \mathrm{E}-04\end{array}$} \\
\hline & & $2.15 \mathrm{E}+01 \pm 6.57 \mathrm{E}-02$ & $2.39 \mathrm{E}+01 \pm 1.20 \mathrm{E}-01$ & $2.37 \pm 4.8 \mathrm{E}-02$ & -2.09 & \\
\hline & & $2.21 \mathrm{E}+01 \pm 2.25-01$ & $2.76 \mathrm{E}+01 \pm 1.58 \mathrm{E}-01$ & $5.24 \pm 1.3 \mathrm{E}-02$ & -1.25 & \\
\hline & \multirow{3}{*}{$m i R-137^{k o / D f}$} & $2.36 \mathrm{E}+01 \pm 2.22 \mathrm{E}-01$ & $2.79 \mathrm{E}+01 \pm 2.63 \mathrm{E}-01$ & $4.00 \pm 4.5 \mathrm{E}-02$ & -0.61 & \multirow{3}{*}{$\begin{array}{c}2.10 \pm 1.1 \mathrm{E}-01 \\
P=1.577 \mathrm{E}-03 \\
P=6.426 \mathrm{E}-02\end{array}$} \\
\hline & & $2.11 \mathrm{E}+01 \pm 1.58 \mathrm{E}-0 \mathrm{q}$ & $2.41 \mathrm{E}+01 \pm 1.22 \mathrm{E}-01$ & $2.99 \pm 2.5 \mathrm{E}-02$ & -1.47 & \\
\hline & & $2.06 \mathrm{E}+01 \pm 2.33-01$ & $2.59 \mathrm{E}+01 \pm 4.66 \mathrm{E}-02$ & $5.35 \pm 1.1 \mathrm{E}-01$ & -1.13 & \\
\hline \multirow{16}{*}{ 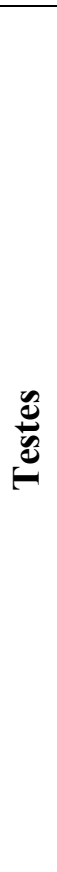 } & \multirow{3}{*}{$w^{1118} / O R$} & $2.08 \mathrm{E}+01 \pm 2.49 \mathrm{E}-01$ & $2.36 \mathrm{E}+01 \pm 2.22 \mathrm{E}-01$ & $1.63 \mathrm{E} \pm 7.77 \mathrm{E}-02$ & 0.01 & \multirow[t]{3}{*}{$1.000 \pm 7.35 \mathrm{E}-02$} \\
\hline & & $2.23 \mathrm{E}+01 \pm 3.05 \mathrm{E}-01$ & $2.42 \mathrm{E}+01 \pm 3.68 \mathrm{E}-01$ & $1.46 \mathrm{E} \pm 2.13 \mathrm{E}-02$ & 0.17 & \\
\hline & & $2.37 \mathrm{E}+01 \pm 4.19 \mathrm{E}-01$ & $2.53 \mathrm{E}+01 \pm 3.86 \mathrm{E}-01$ & $1.58 \mathrm{E} \pm 9.66 \mathrm{E}-02$ & 0.05 & \\
\hline & \multirow{3}{*}{$m i R-137^{k o}$} & $2.24 \mathrm{E}+01 \pm 2.36 \mathrm{E}-01$ & $2.37 \mathrm{E}+01 \pm 2.24 \mathrm{E}-01$ & $1.31 \pm 9.13 \mathrm{E}-02$ & -0.33 & \multirow{3}{*}{$\begin{array}{c}3.96 \pm 3.36 \mathrm{E}-02 \\
P=1.020 \mathrm{E}-02\end{array}$} \\
\hline & & $2.13 \mathrm{E}+01 \pm 2.88 \mathrm{E}-01$ & $2.29 \mathrm{E}+01 \pm 2.43 \mathrm{E}-01$ & $1.53 \pm 1.61 \mathrm{E}-02$ & -0.05 & \\
\hline & & $2.63 \mathrm{E}+01 \pm 3.02 \mathrm{E}-01$ & $2.75 \mathrm{E}+01 \pm 1.34 \mathrm{E}-01$ & $1.08 \pm 3.76 \mathrm{E}-02$ & -0.40 & \\
\hline & \multirow{3}{*}{$m i R-137^{k o / D f}$} & $2.25 \mathrm{E}+01 \pm 3.23 \mathrm{E}-01$ & $2.51 \mathrm{E}+01 \pm 3.03 \mathrm{E}-01$ & $2.62 \pm 5.83 \mathrm{E}-02$ & 0.99 & \multirow{3}{*}{$\begin{array}{c}1.67 \pm 2.25 \mathrm{E}-02 \\
P=5.580 \mathrm{E}-03 \\
P=2.035 \mathrm{E}-03\end{array}$} \\
\hline & & $2.22 \mathrm{E}+01 \pm 2.51 \mathrm{E}-01$ & $2.55 \mathrm{E}+01 \pm 3.35 \mathrm{E}-01$ & $3.33 \pm 5.09 \mathrm{E}-02$ & 1.64 & \\
\hline & & $2.46 \mathrm{E}+01 \pm 2.46 \mathrm{E}-02$ & $2.73 \mathrm{E}+01 \pm 1.29 \mathrm{E}-02$ & $2.68 \pm 8.01 \mathrm{E}-03$ & 1.05 & \\
\hline & \multirow{3}{*}{$t j>$ scramble sponge $^{\text {spe }}$} & $2.19 \mathrm{E}+01 \pm 3.58 \mathrm{E}-01$ & $2.32 \mathrm{E}+01 \pm 3.08 \mathrm{E}-01$ & $2.01 \pm 4.49 \mathrm{E}-02$ & -0.07 & \multirow[t]{3}{*}{$1.000 \pm 6.45 \mathrm{E}-02$} \\
\hline & & $2.15 \mathrm{E}+01 \pm 2.72 \mathrm{E}-01$ & $2.35 \mathrm{E}+01 \pm 4.74 \mathrm{E}-01$ & $2.14 \pm 1.52 \mathrm{E}-01$ & -0.06 & \\
\hline & & $2.35 \mathrm{E}+01 \pm 2.59 \mathrm{E}-01$ & $2.53 \mathrm{E}+01 \pm 4.41 \mathrm{E}-02$ & $2.09 \pm 1.39 \mathrm{E}-01$ & -0.01 & \\
\hline & \multirow{3}{*}{$D g^{1.10 G}:: t j>m i R-137^{\text {sponge }}$} & $2.18 \mathrm{E}+01 \pm 2.57 \mathrm{E}-01$ & $2.35 \mathrm{E}+01 \pm 3.40 \mathrm{E}-01$ & $1.64 \pm 4.01 \mathrm{E}-02$ & -0.37 & \multirow{3}{*}{$\begin{array}{c}1.376 \pm 3.54 \mathrm{E}-02 \\
P=2.743 \mathrm{E}-03 \\
P=3.926 \mathrm{E}-03\end{array}$} \\
\hline & & $2.18 \mathrm{E}+01 \pm 2.46 \mathrm{E}-02$ & $2.35 \mathrm{E}+01 \pm 1.29 \mathrm{E}-02$ & $1.73 \pm 4.78 \mathrm{E}-02$ & -0.40 & \\
\hline & & $1.69 \mathrm{E}+01 \pm 3.10 \mathrm{E}-02$ & $1.84 \mathrm{E}+01 \pm 4.52 \mathrm{E}-02$ & $1.48 \pm 1.48 \mathrm{E}-02$ & -0.60 & \\
\hline & $t j>m i R-137^{\text {sponge }}$ & $2.46 \mathrm{E}+01 \pm 4.14 \mathrm{E}-02$ & $2.63 \mathrm{E}+01 \pm 1.29 \mathrm{E}-02$ & $2.46 \pm 4.66 \mathrm{E}-02$ & 0.45 & $2.33 \pm 1.23 \mathrm{E}-02$ \\
\hline
\end{tabular}




\begin{tabular}{|c|c|c|c|c|c|}
\hline & $2.16 \mathrm{E}+01 \pm 3.34 \mathrm{E}-01$ & $2.47 \mathrm{E}+01 \pm 5.35 \mathrm{E}-01$ & $3.18 \pm 1.16 \mathrm{E}-01$ & 1.04 & $P=1.734 \mathrm{E}-02$ \\
\hline & $2.19 \mathrm{E}+01 \pm 3.38 \mathrm{E}-01$ & $2.48 \mathrm{E}+01 \pm 5.35 \mathrm{E}-01$ & $3.18 \pm 1.11 \mathrm{E}-01$ & 1.09 & \multirow{4}{*}{$\begin{array}{c}5.68 \pm 1.48 \mathrm{E}-02 \\
P=2.582 \mathrm{E}-04 \\
P=7.645 \mathrm{E}-03\end{array}$} \\
\hline \multirow{3}{*}{$t j>D g$} & $2.28 \mathrm{E}+01 \pm 2.63 \mathrm{E}-01$ & $2.44 \mathrm{E}+01 \pm 5.05 \mathrm{E}-01$ & $1.47 \pm 1.43 \mathrm{E}-01$ & -0.55 & \\
\hline & $2.32 \mathrm{E}+01 \pm 3.89 \mathrm{E}-02$ & $2.55 \mathrm{E}+01 \pm 5.52 \mathrm{E}-01$ & $1.53 \pm 7.56 \mathrm{E}-02$ & -0.60 & \\
\hline & $2.29 \mathrm{E}+01 \pm 2.33 \mathrm{E}-02$ & $2.49 \mathrm{E}+01 \pm 5.01 \mathrm{E}-02$ & $1.97 \pm 7.56 \mathrm{E}-02$ & -0.12 & \\
\hline
\end{tabular}

Values obtained for averages of three biological replicates. Error bars represent AVE \pm SEM, and statistical significance was determined by twotailed Student's t-test $\left({ }^{*} \mathrm{P}<0.05,{ }^{*} \mathrm{P}<0.01, * * * \mathrm{P}<0.001\right)$. 
Supplementary Table 7. Early somatic cell counts per testes

\begin{tabular}{|l|c|c|c|c|l|}
\hline Genotype & $\begin{array}{l}\mathbf{T j}^{+} \text {cells } \\
\text { AVE } \pm \text { SEM }\end{array}$ & $\begin{array}{l}\mathbf{E y a}^{+} \text {cells } \\
\text { AVE } \pm \text { SEM }\end{array}$ & $\begin{array}{l}\mathbf{( T j + E y a ) ~}^{+} \text {cells } \\
\text { AVE } \pm \text { SEM }\end{array}$ & $\begin{array}{l}\text { Total cells } \\
\text { AVE } \pm \text { SEM }\end{array}$ & $\mathbf{n}$ \\
\hline \multirow{2}{*}{$w^{1118} / O R$} & 56.322 & 84.011 & 6.389 & 134.253 & 24 \\
& \pm 2.023 & \pm 1.204 & \pm 0.405 & \pm 3.054 & \\
\hline \multirow{2}{*}{$m i R-137^{k o}$} & 82.204 & 80.438 & 11.062 & 151.581 & 24 \\
& \pm 1.488 & \pm 0.451 & \pm 1.953 & \pm 0.163 & \\
& $P=1.381 \mathrm{E}-02$ & $P=1.885 \mathrm{E}-01$ & $P=2.246 \mathrm{E}-01$ & $P=5.566 \mathrm{E}-02$ & \\
\hline
\end{tabular}

Two-tailed Student's t-test with averages of three independent biological replicates was used to determine $\mathrm{P}$ values. ${ }^{*} \mathrm{P}<0.05,{ }^{*} \mathrm{P}<0.01,{ }^{* * *} \mathrm{P}<0.001$.

$\mathrm{n}=$ number of testes analyzed

Supplementary Table 8. Early somatic cell counts at the apical portion of testes

\begin{tabular}{|l|l|l|l|}
\hline Genotype & $\begin{array}{l}\text { Tj-positive } \\
\text { cells }\end{array}$ & $\mathbf{n}$ & $\boldsymbol{P}$ value \\
\hline$w 1118 / O R$ & $43.800 \pm 1.110$ & 32 & - \\
\hline miR-137ko & $68.559 \pm 2.909$ & 34 & $1.337 \mathrm{E}-03$ \\
\hline miR-137ko/Df & $36.721 \pm 2.849$ & 31 & $7.736 \mathrm{E}-01$ \\
\hline$t j / w^{1118}$ & $52.877 \pm 2.133$ & 28 & - \\
\hline$t j>D g$ & $42.150 \pm 1.520$ & 22 & $8.093 \mathrm{E}-02$ \\
\hline$t j>m i R-137$ & $50.236 \pm 0.944$ & 27 & $7.736 \mathrm{E}-01$ \\
\hline$t j>$ scramblesponge & $39.155 \pm 1.600$ & 35 & - \\
\hline$t j>m i R-137$ sponge & $70.939 \pm 4.521$ & 37 & $3.845 \mathrm{E}-02$ \\
\hline $\begin{array}{l}\text { Dg1.10G,tj>miR- } \\
137 \text { sponge }\end{array}$ & $39.092 \pm 2.345$ & 29 & $1.317 \mathrm{E}-02$ \\
\hline
\end{tabular}

Two-tailed Student's t-test with averages of three independent biological replicates was used to determine $\mathrm{P}$ values. ${ }^{*} \mathrm{P}<0.05, * * \mathrm{P}<0.01, * * * \mathrm{P}<0.001$.

$\mathrm{n}=$ number of testes analyzed 
Supplementary Table 9. Percentage of permeable testes

\begin{tabular}{|c|c|c|c|}
\hline Genotype & \% of permeable testes (AVE \pm SEM) & n & P value \\
\hline Control & $27.92 \pm 2.5$ & 46 & - \\
\hline$m i R-137^{k o}$ & $88.51 \pm 1.46$ & 40 & $1.57 \mathrm{E}-06$ \\
\hline$m i R-137^{k o / D f}$ & $37.78 \pm 4.70$ & 26 & $4.60 \mathrm{E}-04$ \\
\hline$D g^{l .10 G}$ & $85.94 \pm 3.47$ & 31 & $2.6 \mathrm{E}-05$ \\
\hline$t j>$ wl118 & $38.83 \pm 1.09$ & 41 & - \\
\hline$t j>D g$ & $69.40 \pm 4.0$ & 32 & $5.88 \mathrm{E}-03$ \\
\hline$t j>m i R-137$ & $41.26 \pm 0.62$ & 39 & $2.40 \mathrm{E}-01$ \\
\hline$m i R-137^{k o / k o}, t j>m i R-137$ & $48.48 \pm 4.67$ & 33 & $1.35 \mathrm{E}-03$ \\
\hline$t j>s c r a m b l e^{\text {sponge }}$ & $38.89 \pm 7.48$ & 30 & - \\
\hline$t j>m i R-137^{\text {sponge }}$ & $72.50 \pm 0.41$ & 40 & $2.85 \mathrm{E}-02$ \\
\hline$D g^{l .10 G}, t j>m i R 137^{\text {sponge }}$ & $49.47 \pm 2.55$ & 24 & $3.46 \mathrm{E}-03$ \\
\hline
\end{tabular}

Two-tailed Student's t-test with averages of three independent biological replicates was used to determine $\mathrm{P}$ values. $* \mathrm{P}<0.05, * * \mathrm{P}<0.01, * * * \mathrm{P}<0.001$.

$\mathrm{n}=$ number of testes analyzed

Supplementary Table 10. SJ counts and morphology on elongated spermatids

\begin{tabular}{|c|c|c|c|}
\hline Genotype & SJ counts (AVE \pm SEM) & \% of abnormal SJ & n \\
\hline Control & $18.253 \pm 1.214$ & $18.264 \pm 0.024$ & 59 \\
\hline$m i R-137^{k o}$ & $17.309 \pm 2.154$ & $51.540 \pm 0.078$ & 45 \\
& $P=8.379 \mathrm{E}-01$ & $P=1.542 \mathrm{E}-03$ & \\
\hline$m i R-137^{k / D f o}$ & 21.583 & 30.685 & 8 \\
\hline$t j>$ scramble ${ }^{\text {sponge }}$ & $22.759 \pm 2.059$ & $15.184 \pm 0.006$ & 22 \\
\hline$t j>m i R-137^{\text {sponge }}$ & $18.909 \pm 0.545$ & $48.446 \pm 0.070$ & 21 \\
& $P=1.00 \mathrm{E}-03^{\mathrm{a}}$ & $P=1.00 \mathrm{E}-03^{\mathrm{a}}$ & \\
\hline$t j>$ w1118 & 17.438 & 16.076 & 16 \\
\hline$t j>m i R-137$ & 16.500 & 18.002 & 12 \\
\hline
\end{tabular}

Two-tailed Student's t-test with averages of three independent biological replicates was used to determine $\mathrm{P}$ values. ${ }^{*} \mathrm{P}<0.05, * * \mathrm{P}<0.01, * * * \mathrm{P}<0.001$.

a $\chi^{2}$ test with Yate's correction in 1 degree of freedom was analyzed to calculate significance. $* \mathrm{P}<0.05, * * \mathrm{P}<0.01, * * * \mathrm{P}<0.001$.

$\mathrm{n}=$ number of testes analyzed 


\section{Appendix}

\section{List of abbreviations}

\section{Technical abbreviations}

A

AVE

BCA

bp

BSA

$\mathrm{C}_{\mathrm{T}}$

DAPI

DGRC

DSHB

ECL

EDTA

FISH

NGS

$\mathrm{nt}$

PBS

PBT

PBTB

PCR

PFA

qPCR

qRT-PCR

Rpm

RT

RT

S2 cells

SD

SEM

ampere

average

bicinchoninic acid

base pair

bovine serum albumin

threshold cycle

4', 6-diamidino-2-phenylindole

Drosophila Genomics Resource Center

Developmental Studies Hybridoma Bank

enhanced chemiluminescence

ethylenediaminetetraacetic acid

fluorescence in situ hybridization

normal goat serum

nucleotide

phosphate buffered saline

PBS-Tween (buffer)

PBT-blocking (buffer)

polymerase chain reaction

paraformaldehyde

quantitative PCR

quantitative reverse transcription PCR

revolutions per minute

reverse transcriptase

room temperature

Schneider 2 cells

standard deviation

Tris tris-hydroxymethyl-aminomethane 


\section{Biological abbreviations}

AMPK AMP-activated protein kinase

A-P axis anterior-posterior axis

BTB blood-testis barrier

C-terminus carboxyl-terminus

cDNA complementary DNA

DNAse deoxyribonuclease

CySCs somatic stem cells

DGC Dystrophin-associated Glycoprotein Complex

Dme Drosophila Melanogaster

DNA deoxyribonucleic acid

Dre Danio rerio

Drosophila Drosophila melanogaster

dsRBD double stranded RNA binding domain protein

E.Coli Escherichia coli

ECM extracellular matrix

GB gonial blast

GO Gene Ontology

GSCs germline stem cells

hsa homo sapiens

mGFP membrane GFP

miRNA microRNA

mти mus musculus

mRNA messenger RNA

MTJ myotendinos junction

PKA cAMP-dependent protein kinase

Pol polymerase

Pre-miRNA precursor microRNA

Pri-miRNA primary microRNA

RNA ribonucleic acid

RISC RNA-induced silencing complex

RNAi RNA interference

RNase ribonuclease 


$\begin{array}{ll}\text { rRNA } & \text { ribosomal RNA } \\ \text { SiRNA } & \text { small interfering RNA } \\ \text { SV } & \text { seminal vesicle } \\ \text { UAS } & \text { upstream activation sequence } \\ \text { UTR } & \text { untranslated region }\end{array}$

\section{Gene and protein names}

act

actin

Add adducin

Arm armadillo

Cora coracle

Chic chickadee

Dg dystroglycan

Dlg discs large

Dys dystrophin

Eya eyes absent

GluR glutamate receptor IIA

Hts hu lo tai shao

How held out wings

LanA/B laminin A

Mega mega trachea

Mei-P26 meiotic P26

MHC myosin heavy chain

mir-310s refers to $m i R-310, m i R-311, m i R-312$, and $m i R-313$ together

miR-975C refers to $m i R-975, m i R-976, m i R-977$ together

miR-959C refers to $m i R-959, m i R-960, m i R-961, m i R-962$ together

Nrx-IV neurexin IV

nNOS nitric oxide synthase

PH3 Phospho-Histone H3

Scg sarcoglycan

Syn1 syntrophin-like 1

$\mathrm{Tj} \quad$ traffic jam

Yki yorkie 
Zfh-1 $\quad$ zinc finger protein 1

\section{Symbols}

$\begin{array}{ll}{ }^{\circ} \mathrm{C} & \text { degree celsius } \\ \mathrm{CO}_{2} & \text { carbon dioxide } \\ \mathrm{G} & \text { gram } \\ \mu \mathrm{g} & \text { microgram } \\ \mu \mathrm{l} & \text { microliter } \\ \mu \mathrm{M} & \text { micromolar } \\ \mathrm{h} & \text { hour } \\ \mathrm{kbp} & \text { kilo base pair } \\ \mathrm{kDa} & \text { kilodalton } \\ \mathrm{Mb} & \text { mega base } \\ \mathrm{ml} & \text { milliliter } \\ \mathrm{min} & \text { minute } \\ \mathrm{mM} & \text { milli molar } \\ \mathrm{NaCl} & \text { sodium chloride } \\ \mathrm{NaH}{ }_{2} \mathrm{PO}_{4} & \text { monosodium phosphate } \\ \mathrm{Na} 2 \mathrm{HPO}_{4} & \text { disodium phophate } \\ \mathrm{NaOH} & \text { sodium hydroxide } \\ \mathrm{ng} & \text { nanogram } \\ \mathrm{V} & \text { volt }\end{array}$




\section{Acknowledgement}

Foremost, I would like to thank my supervisor Halyna R. Shcherbata for giving me an opportunity to peruse my $\mathrm{PhD}$ under her supervision. Her continuous guidance, suggestions, and challenges has shaped me to be a better student in these 3.5 years. I not only got to realize what it takes to be a good scientist, but also a successful PI and a great human being. Some of the life lessons I took watching her closely will aspire me to be better in many ways in the years to come.

Next, I would like to thank my thesis committee members Stefan Bonn and Jörg Großhans for their suggestions, ideas, criticism and support for making this project a success. I would like to especially thank Stefan Bonn for always supporting my project and being available whenever I needed his suggestions. I would like to thank Roland Dosch, Martin Göpfert and Ahmed Mansouri for joining as my extended committee members.

My sincere gratitude to my current and previous lab mates who gave me their valuable scientific input, technical support, and especially for their company, creating a family like environment. Travis, for always motivating and helping me in every possible way to make me focus and laugh even at times of doubts and confusions. For more being a friend than just a colleague. You kept me optimistic during my times of discouragement and made weekends work a happy place to be. Ömer, for sharing his experiences as a graduating $\mathrm{PhD}$ student and answering all my concerns during the initial phase of my project. Mariya, for teaching me tools and techniques of the lab during my time as an intern and making me familiar with the department. Andriy, for all the help and support during the entire project. Rucha, for helping me during the final phase of my project. Jasmine, for creating Dg ${ }^{1.10 \mathrm{G}}$ mutants that came handy on this project. To the whole Herbert Jäckle Department for their input during departmental seminars, support through reagents and technical contributions. My students, Tadas, Liezel, Daniel, and Alejandra who helped me in my project. I got an opportunity to grow as a mentor and learned a lot from them.

GGNB for giving an opportunity to learn many aspects of science. I have benefited a lot from scientific and soft skill courses throughout these years. I have enjoyed being their student representative, editorial board in GGNB times and as a part of organizing committee for WoCaNet symposium. I have come to realize many of my strengths and weakness throughout the exposures I got with these activities. I would like to acknowledge the travel grant that allowed me to present my work in international symposium. 
To my friends in Göttingen, for adding healthy dosage of laughter and craziness throughout these years. Prajwal, for always listening, making tea breaks more compelling, sharing enthusiasm and frustrations and adding positivity in everyday life. Shoba, for exciting conversations about science, career and for crazy moments turning Göttingen a familiar place to be.

To my near and dear friend, Elizabeth, for making my adaptation easier since my first day in Germany. Our long conversations, life experiences always lit positivity in me. Manita, for listening to my worries and fruitful tips and a continuous reminder to taking care of myself. My childhood buddies (RSKDM), for teaching me a meaning of friendship for past 18 years. Losing one of you during these years was a shock and a realization of life beyond dreams and career.

To my family, my parents for believing in me, for always being enthusiastic to see me becoming a Doctor despite of not knowing either my project or the nature of my research. My sisters, who are my backbone. They believed so much in me that they never let me fail. Especially Priti, for taking care of our parents and home all these years. Kriti, for always encouraging me to stay on my track. My in-laws, for always motivating me to give my best. I think I stressed them more than I stressed myself during the last phase of my project. To Bhupesh and Semi for their support and accepting my absence during family engagements. To my husband Bhawesh, my life support, for his continuous support, love and for keeping up with me throughout these years. For taking care of our home, my smallest needs, for not giving up on me and not letting me give up. You truly deserve a share of my Doctor title. 